\title{
Energy Information Administration
}

\section{Costs and Indices for}

Domestic Oil and Gas Field Equipment and Production Operations 1992 Through 1995 


\section{HOW TO OBTAIN EIA PRODUCTS AND SERVICES}

For further information on any of the following services, or for answers to energy information questions, please contact EIA's National Energy Information Center:
National Energy Information Center (NEIC)
(202) $586-8800$
Energy Information Administration
(202) 586-0727 (fax)
Forrestal Building, Room 1F-048
TTY: (202) 586-1181
Washington, DC 20585
E-mail: infoctr@eia.doe.gov

\section{Electronic Products and Services}

EIA's Internet Site Services offer nearly all EIA publications. Users can view and download selected pages or entire reports, search for information, download EIA data and analysis applications, and find out about new EIA information products and services.

\section{World Wide Web: http://www.eia.doe.gov Gopher: gopher://gopher.eia.doe.gov \\ FTP: ftp://ftp.eia.doe.gov}

EIA also offers a listserve service for EIA press releases and other short documents. Sign up on the EIA World Wide Web site.

EIA's CD-ROM, Energy InfoDisc, contains most EIA publications, several databases, and an energy forecasting model. The Energy InfoDisc, produced quarterly, is available for a fee from STAT-USA, Department of Commerce, 1-800-STAT-USA.

The Comprehensive Oil and Gas Information Source (COGIS), a bulletin board service, contains data files from most of EIA's oil- and gas-related reports. It is available for a fee from STAT-USA, on 1-800-STAT-USA.

EIA's Electronic Publishing System (EPUB) bulletin board contains data files, directories, and forecasts from most EIA reports. It can be accessed free of charge by dialing (202) 586-2557.

Many of EIA's data files and modeling programs are available for sale on diskette, tape, or cartridge, through either the National Technical Information Service or the Office of Scientific and Technical Information, Department of Energy. Contact NEIC for information on specific products, sources, and media, and ordering instructions.

\section{Printed Publications}

EIA directories are available free of charge from NEIC. Recent periodicals and one-time reports are available from the Government Printing Office. Older reports are available from the National Technical Information Service:

Superintendent of Documents U.S. Government Printing Office P.O. Box 371954

Pittsburgh, PA 15250-7954 (202) 512-1800; (202)-512-2250 (fax)
National Technical Information Service

U.S. Department of Commerce

Springfield, VA 22161

5285 Port Royal Road

(703) 487-4650; (703) 321-8547 (fax) 


\section{DISCLAIMER}

Portions of this document may be illegible in electronic image products. Images are produced from the best available original document. 
DOE/EIA-0185(95)

Distribution Category UC-950

\title{
Costs and Indices for \\ Domestic Oil and Gas Field Equipment and Production Operations
}

\section{Through 1995}

\author{
August 1996 \\ Energy Information Administration \\ Office of Oil and Gas \\ U.S. Department of Energy \\ Washington D.C. 20585
}

This report was prepared by the Energy Information Administration, the independent statistical and analytical agency within the Department of Energy. The information contained herein should not be construed as advocating or reflecting any policy position of the Department of Energy or any other organization. 
3
3
3

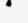




\section{Preface}

This is the Energy Information Administration's (EIA's) thirteenth report in the series on domestic costs and indices for oil and gas field equipment and production operations. The purpose of the series is to provide a continuing means of gauging changes in the oil and gas producing industry's costs. The last report in this series, which reported data from 1990 through 1993, had report number DOE/EIA TR-0568. This report returns to the prior report series nomenclature, DOE/EIA 0185 (95). The cost data presented in this report are used by government agencies, the academic community, and the oil and gas industry. EIA gratefully acknowledges the cooperation received from the personnel of oil and gas service, supply, and production companies throughout the United States, without which this study would not have been possible. General information about this publication may be obtained from Joan Heinkel (202/586-6090), Director of the Reserves and Natural Gas Division, or John Wood (214/767-2200), Director of the Dallas Field Office. Specific information regarding the preparation or contents of this publication may be obtained from Ralph Russell (214/767-2906, rrussell@eia.doe.gov) or Velton Funk (214/767-0884, vfunk@eia.doe.gov), both of whom are petroleum engineers in EIA's Dallas Field Office (fax: 214/767-2204). 


\section{Contents}

Page

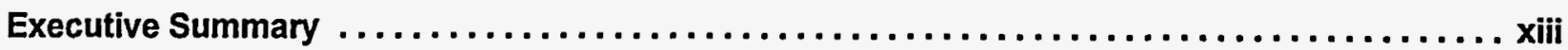

1. Introduction $\ldots \ldots \ldots \ldots \ldots \ldots \ldots \ldots \ldots \ldots \ldots \ldots \ldots \ldots \ldots \ldots \ldots \ldots \ldots \ldots \ldots \ldots \ldots \ldots$

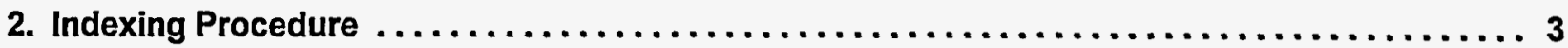

3. Discussion of Results $\ldots \ldots \ldots \ldots \ldots \ldots \ldots \ldots \ldots \ldots \ldots \ldots \ldots \ldots \ldots \ldots \ldots \ldots \ldots \ldots$

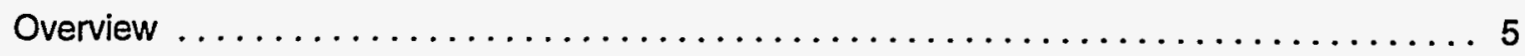

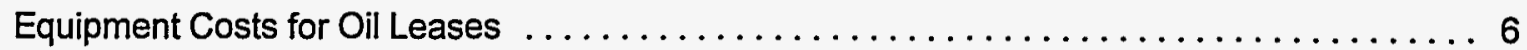

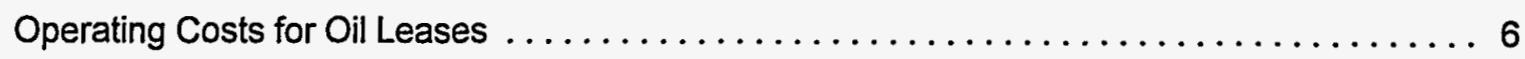

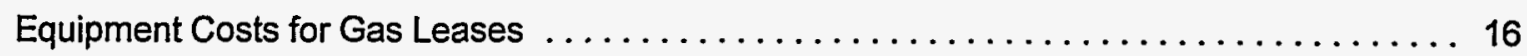

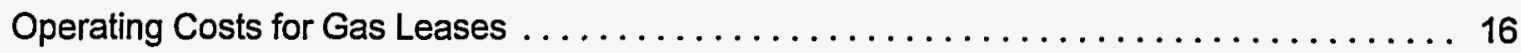

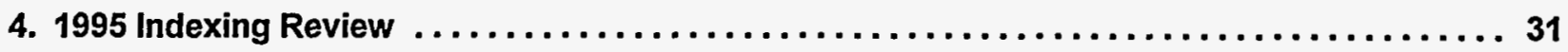

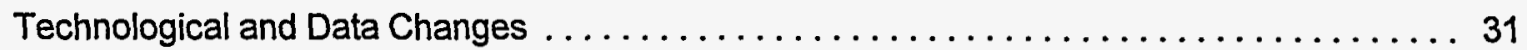

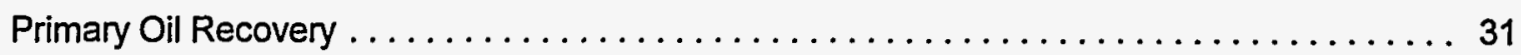

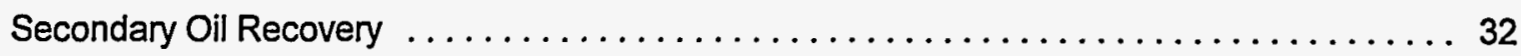

Offshore Gas and Primary Oil Recovery $\ldots \ldots \ldots \ldots \ldots \ldots \ldots \ldots \ldots \ldots \ldots \ldots \ldots \ldots \ldots \ldots$

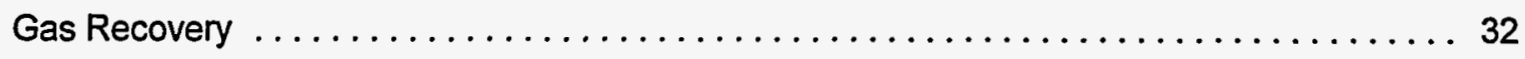

\section{Appendices}

\section{Section I (A through G)}

Costs and Indices for Domestic Oil Field Equipment and Production Operations . . . . . . . 35

\section{Section II ( $\mathrm{H}$ through $\mathrm{M})$}

Costs and Indices for Domestic Gas Field Equipment and Production Operations . . . . . . 71

\section{Section III (N)}

Equipping and Operating Cost Indices and Other Economic Indicators $\ldots \ldots \ldots \ldots \ldots 103$

5. Glossary 


\section{Tables}

1. Summary of Lease Equipment Costs and Composite Indices for Primary Oil Recovery Operations

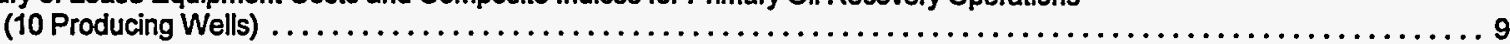

2. Summary of Additional Costs and Composite Indices for Lease Equipment and Injection Wells in West Texas for Secondary Oil Recovery Operations (10 Producing and 11 Injection Wells) $\ldots \ldots \ldots \ldots \ldots \ldots \ldots 11$

3. Summary of Direct Annual Operating Cosis and Composite Indices for Primary Oil Recovery Operations (10 Producing Wells)

4. Summary of Direct Annual Operating Costs and Composite Indices for Secondary Oil Recovery Operations in West Texas (10 Producing and 11 Injection Wells) $\ldots \ldots \ldots \ldots \ldots \ldots \ldots$

5. Summary of Direct Annual Operating Cosis and Composite Indices per Platform - Gulf of Mexico

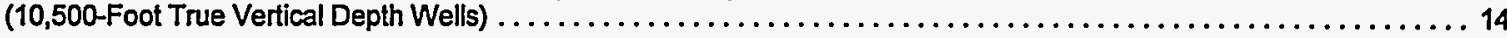

6. Average Equipment Costs and Indices for Gas Leases Aggregated for All Depths, Regions, and

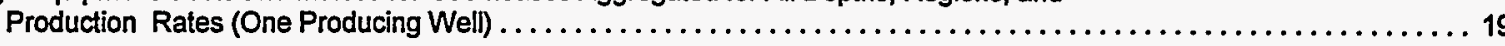

7. Summary of Gas Lease Equipment Costs and Composite Indices

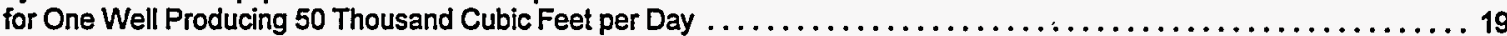

8. Summary of Gas Lease Equipment Costs and Composite Indices for One Well Producing 250 Thousand Cubic Feet per Day

9. Summary of Gas Lease Equipment Costs and Composite Indices for One Well Producing 500 Thousand Cubic Feet per Day

10. Summary of Gas Lease Equipment Costs and Composite Indices

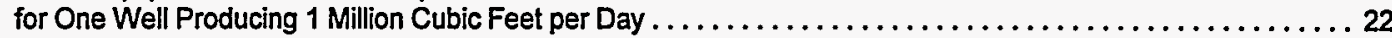

11. Summary of Gas Lease Equipment Costs and Composite Indices for One Well Producing 5 Million Cubic Feet per Day

12. Summary of Gas Lease Equipment Costs and Composite Indices

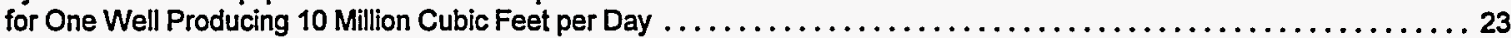

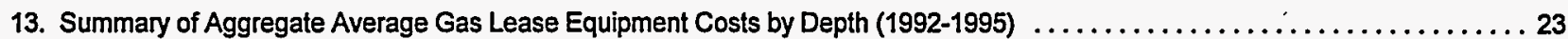

14. Average Operating Costs and Indices for Gas Leases Aggregated for All Depths, Regions, and Production Rates (One Producing Well)

15. Summary of Gas Lease Operating Costs and Composite Indices for One Well Producing 50 Thousand Cubic Feet per Day

16. Summary of Gas Lease Operating Costs and Composite Indices for One Well Producing 250 Thousand Cubic Feet per Day

17. Summary of Gas Lease Operating Costs and Composite Indices for One Well Producing 500 Thousand Cubic Feet per Day

18. Summary of Gas Lease Operating Costs and Composite Indices for One Well Producing 1 Million Cubic Feet per Day .

19. Summary of Gas Lease Operating Costs and Composite Indices

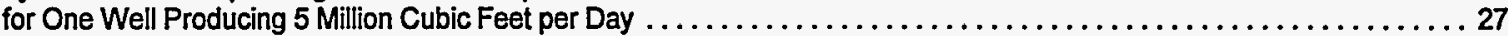

20. Summary of Gas Lease Operating Costs and Composite Indices

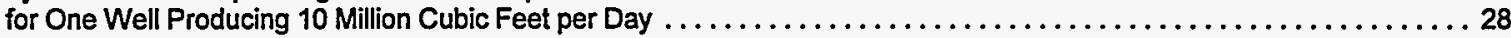

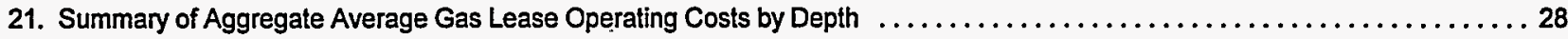

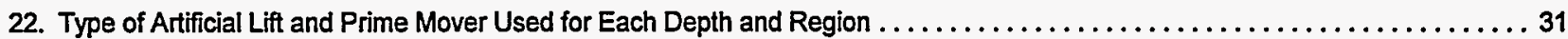




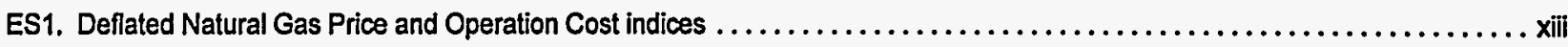

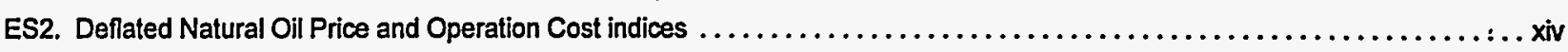

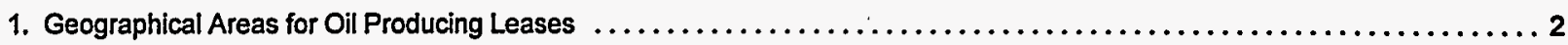

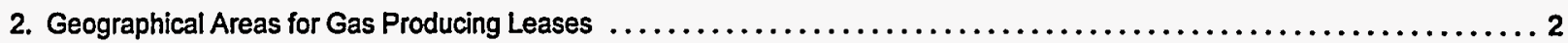

3. Aggregate Average Costs for Primary Oil Recovery, 1976-1995

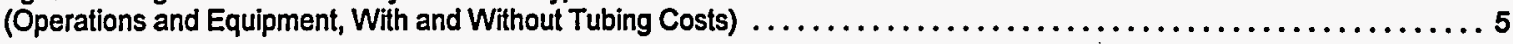

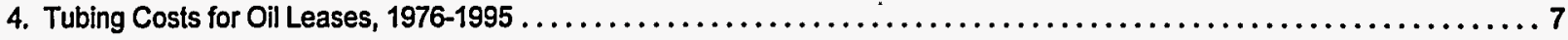

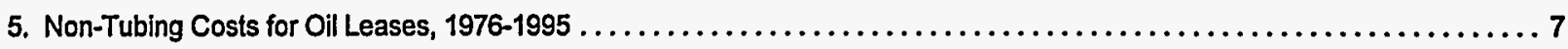

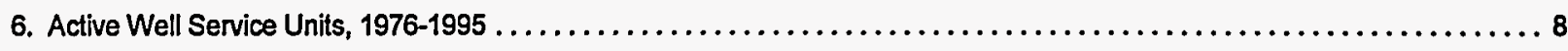

7. Aggregate Average Costs for Gas Recovery, 1976-1995 (Equipment and Operation Cost) ................ 8

8. Aggregate Average Lease Equipment Costs for Primary Oil Recovery, 1992-1995

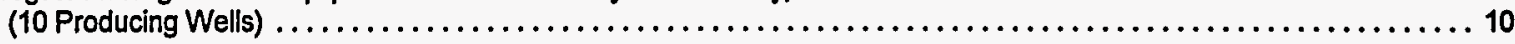

9. Additional Cost of Lease Equipment for Secondary Oil Recovery in West Texas, 1992-1995

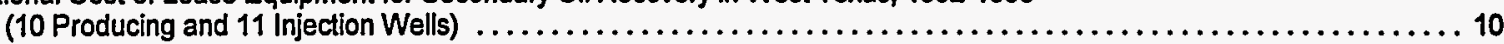

10. Aggregate Annual Operating Costs for Primary Oil Recovery Operations, $1992-1995 \ldots \ldots \ldots \ldots \ldots \ldots \ldots \ldots \ldots \ldots$

11. Annual Operating Costs for Secondary Oil Recovery in West Texas, 1992-1995

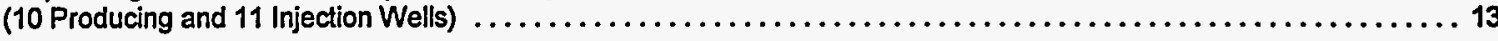

12. Fuel, Power, and Water Cost Indices for 12,000 -foot Oil Wells in California and the Rocky Mountains $\ldots \ldots \ldots \ldots \ldots \ldots$

13. Fuel, Power, and Water Cost Indices for Primary and Secondary Operating Costs

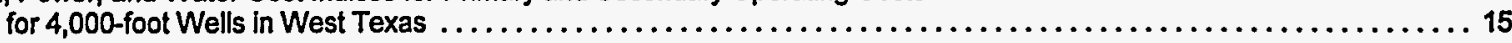

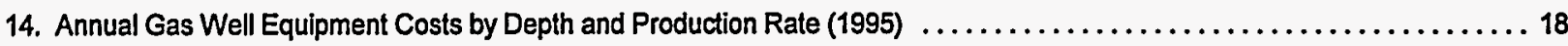

15. Aggregate Average Equipment Costs for a One Well Gas Lease by Production Rate, $1992-1995$. . . . . . . . . . . 18

16. Aggregate Average Annual Gas Well Operating Costs for a One-Well Gas Lease

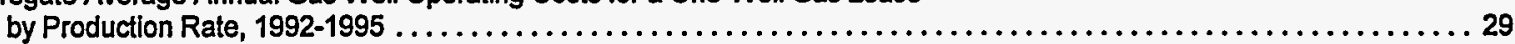

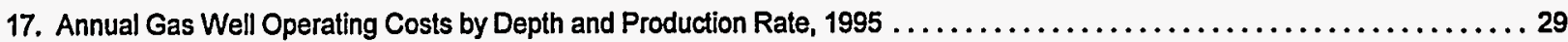

N1. Gross Domestic Product Deflated Producer Price Indices, and Oil and

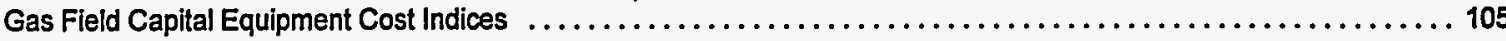

N2. Gross Domestic Product Deflated Operating Cost Indices for Oil and Gas Fields ...................... 105

\section{Appendix A}

Lease Equipment Costs and Indices for Primary Oil Production in West Texas

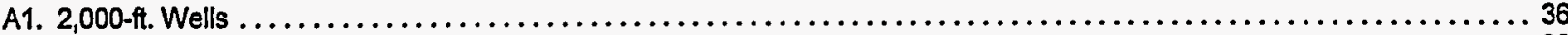

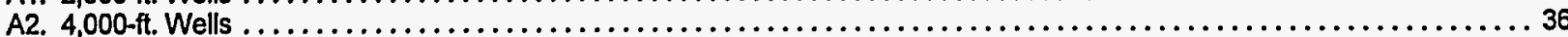

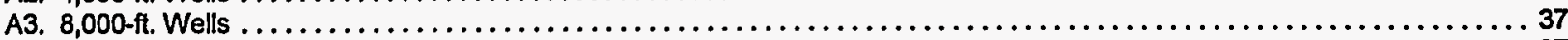

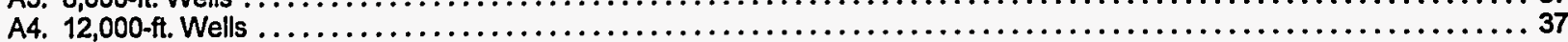

Direct Annual Operating Costs and Indices for Primary Oil Production in West Texas

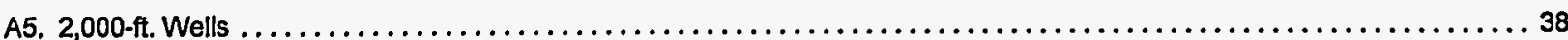

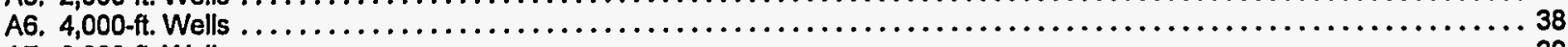

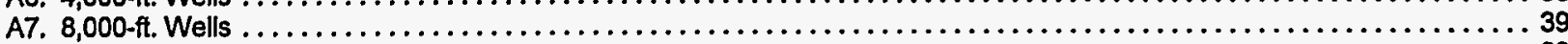

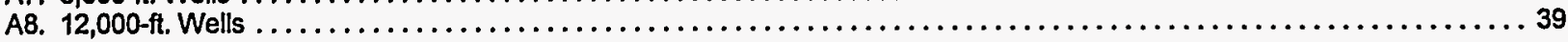


Additional Lease Equipment and Well Costs and Indices for Secondary Oil Production in West Texas

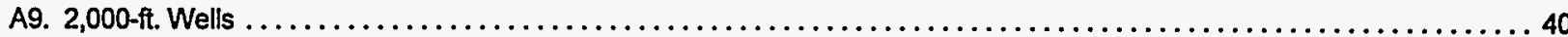

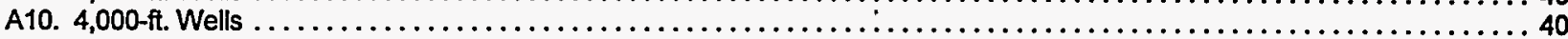

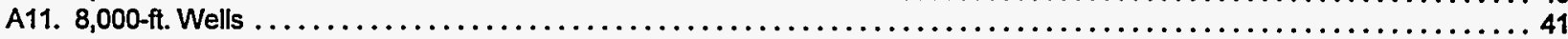

Direct Annual Operating Costs and Indices for Secondary Oil Production in West Texas

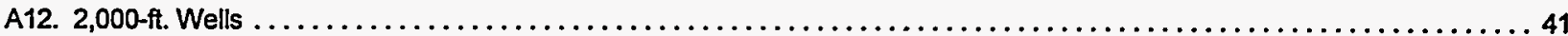

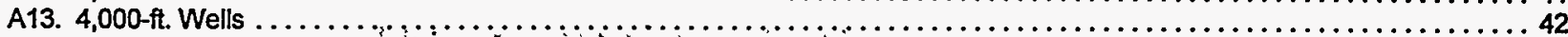

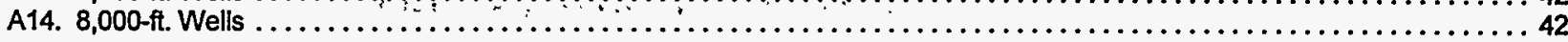

Detailed Equipment List

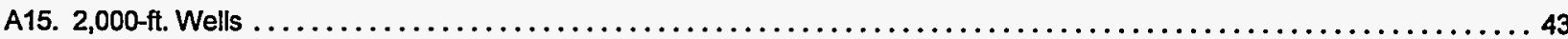

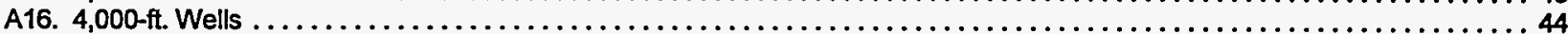

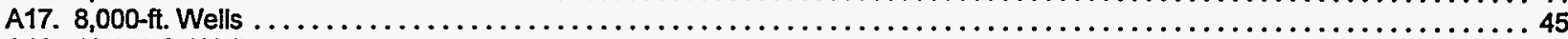

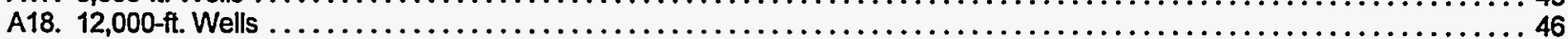

\section{Appendix B}

Lease Equipment Costs and Indices for Primary Oil Production in South Texas

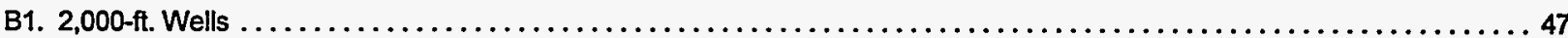

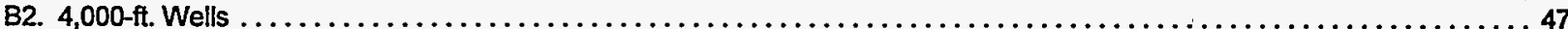

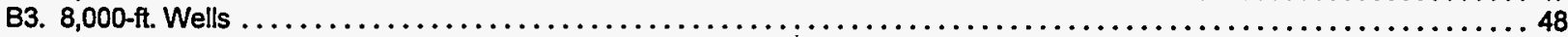

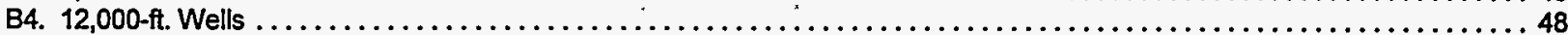

Direct Annual Operating Costs and Indices for Primary Oil Production in South Texas

B5. $2,000-f t$. Wells $\ldots \ldots \ldots \ldots \ldots \ldots \ldots \ldots \ldots \ldots \ldots \ldots \ldots \ldots \ldots \ldots \ldots \ldots \ldots \ldots \ldots \ldots \ldots \ldots \ldots \ldots \ldots \ldots \ldots \ldots \ldots \ldots, 49$

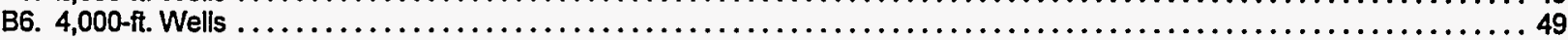

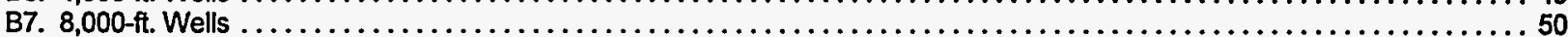

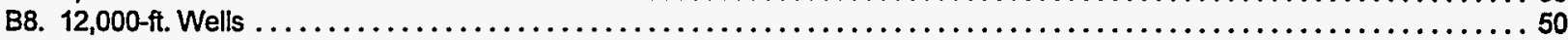

\section{Appendix C}

Lease Equipment Costs and Indices for Primary Oil Production in South Louisiana

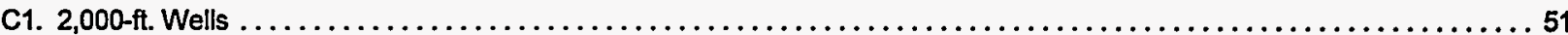

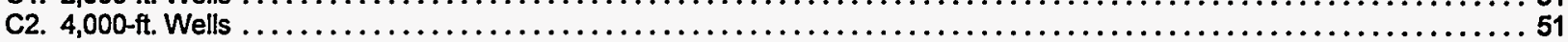

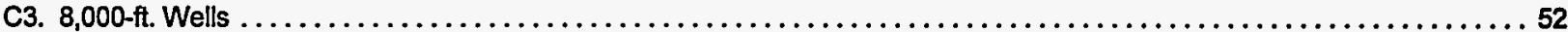

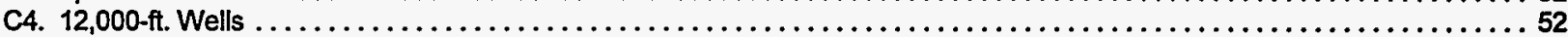

Direct Annual Operating Costs and Indices for Primary Oil Production in South Louisiana

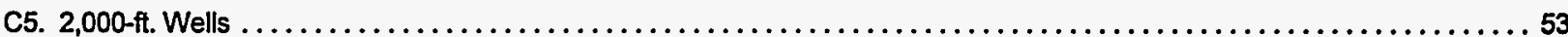

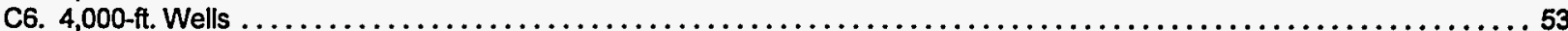

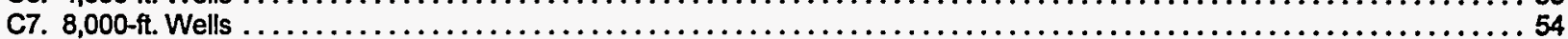

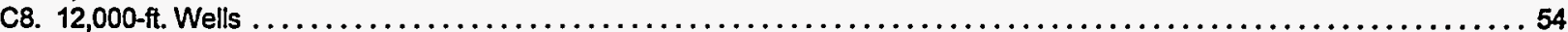

\section{Appendix D}

Lease Equipment Costs and Indices for Primary Oil Production in Oklahoma

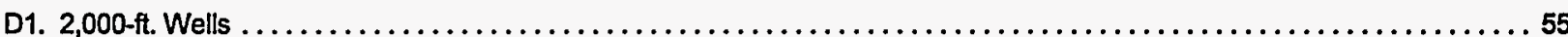

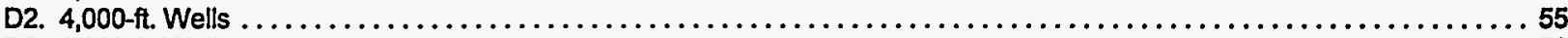

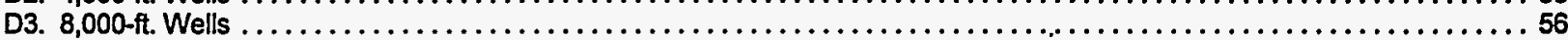

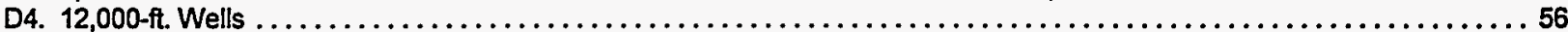

Direct Annual Operating Costs and Indices for Primary Oil Production in Oklahoma

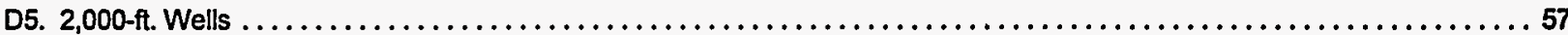

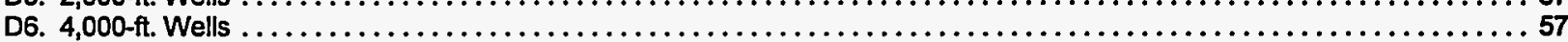

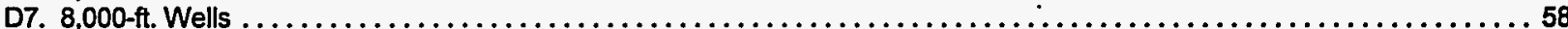

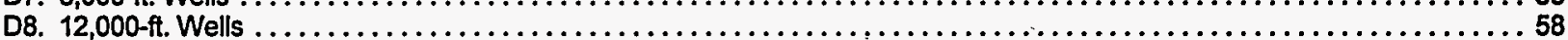




\section{Appendix E}

Lease Equipment Costs and Indices for Primary Oil Production in the Rocky Mountains

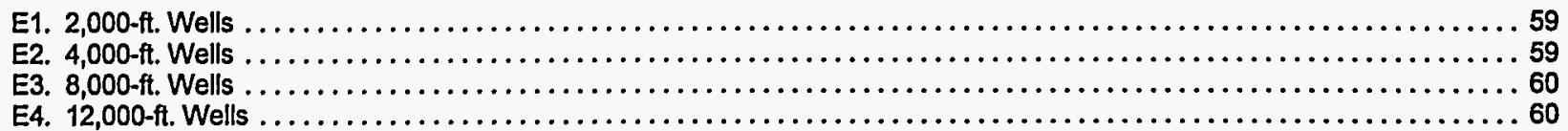

Direct Annual Operating Costs and Indices for Primary Oil Production in the Rocky Mountains

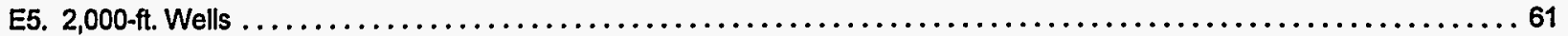

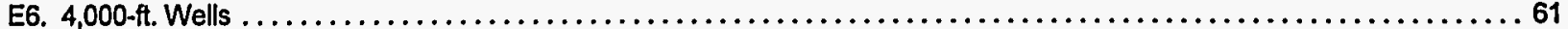

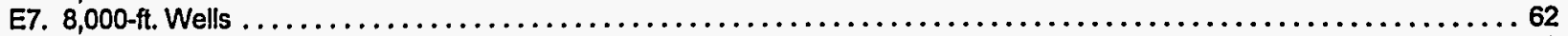

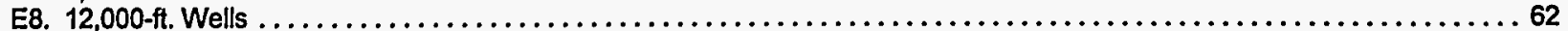

\section{Appendix F}

Lease Equipment Costs and Indices for Primary Oil Production in California

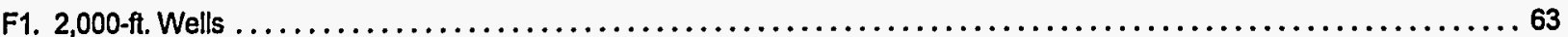

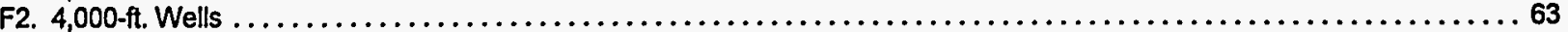

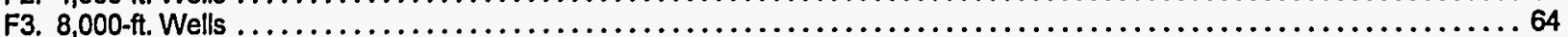

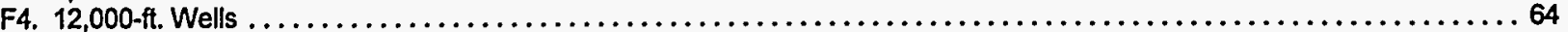

Direct Annual Operating Costs and Indices for Primary Oil Production in Califomia

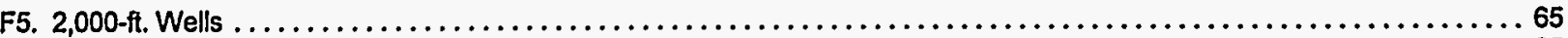

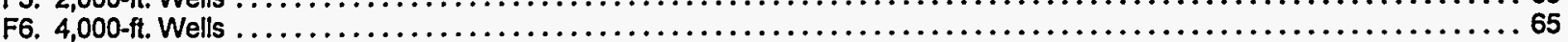

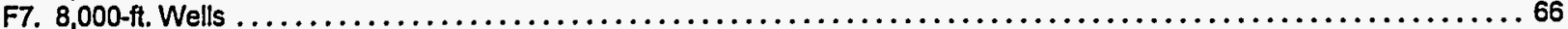

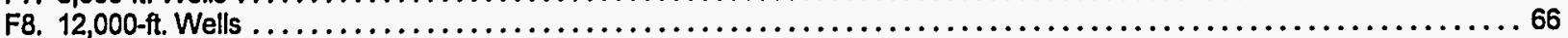

\section{Appendix G}

Annual Operating Costs and Indices for Platforms in the Gulf of Mexico

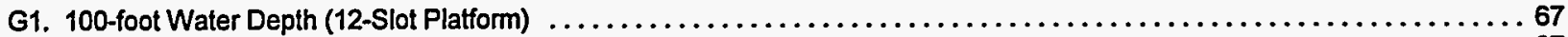

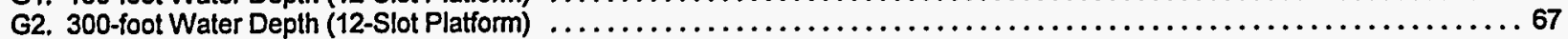

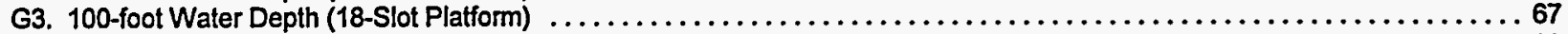

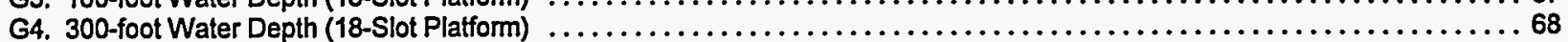

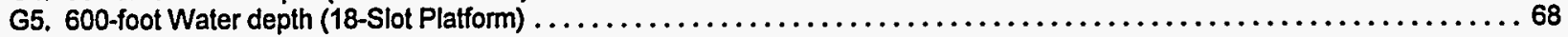

\section{Appendix H}

\section{Lease Equipment Costs and Indices for Gas Production in West Texas}

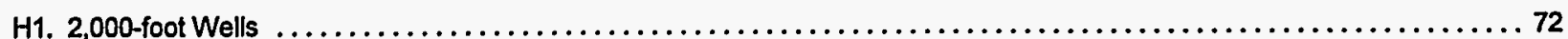

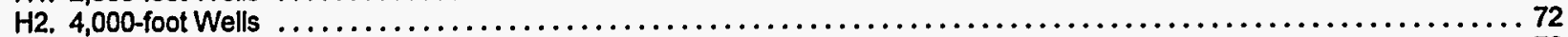

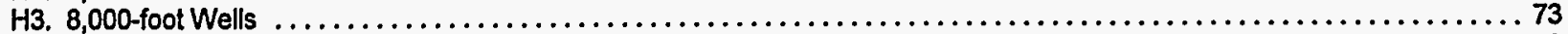

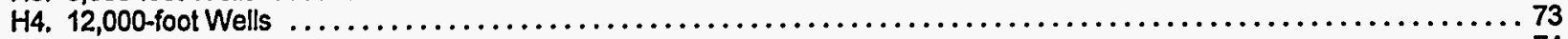

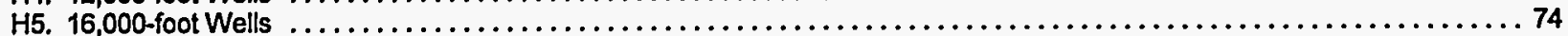

Direct Annual Operating Costs and Indices for Gas Production in West Texas

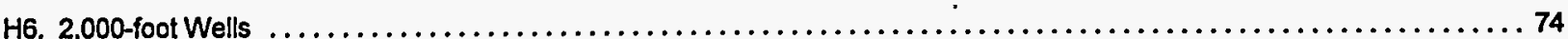

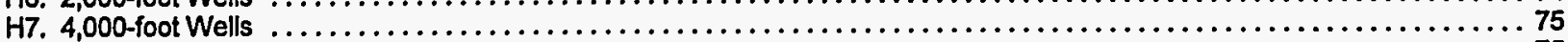

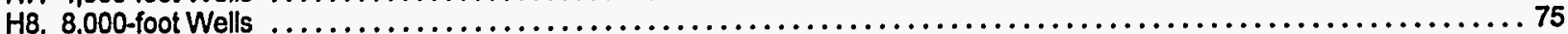

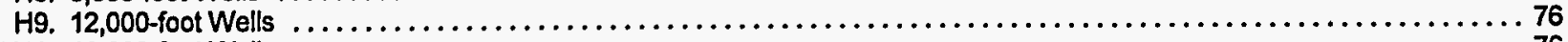

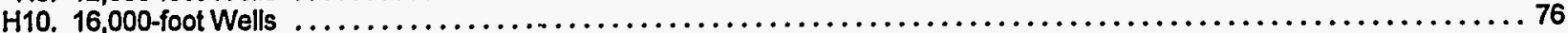

H11. Detailed Lease Equipment List for 12,000-Foot Gas Wells

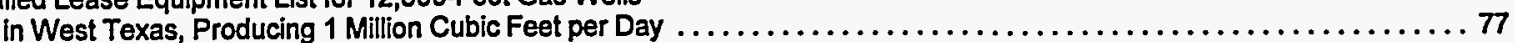

\section{Appendix I}

Lease Equipment Costs and Indices for Gas Production in South Texas

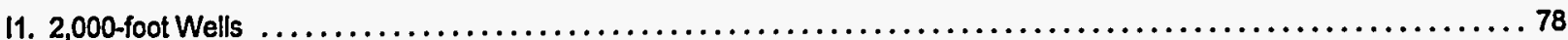

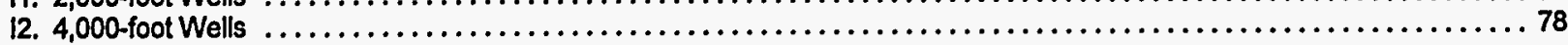




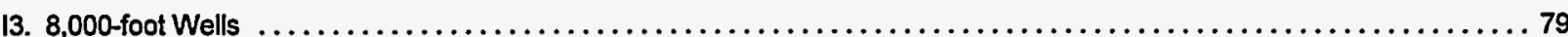

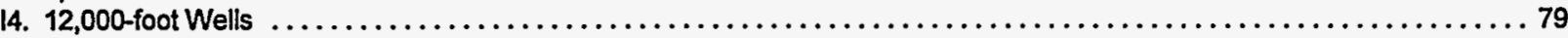

Direct Annual Operating Costs and Indices for Gas Production in South Texas

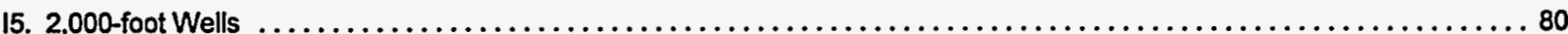

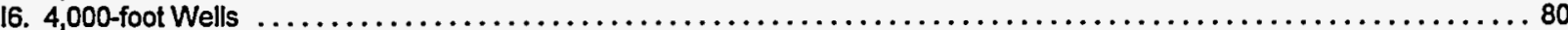

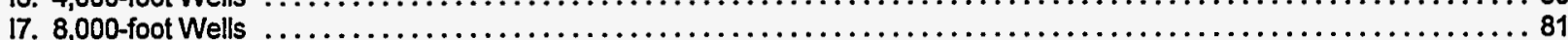

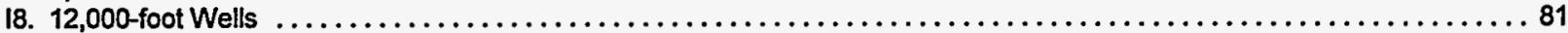

\section{Appendix J}

Lease Equipment Costs and Indices for Gas Production in South Louisiana

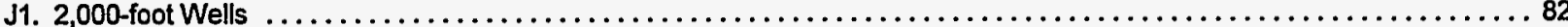

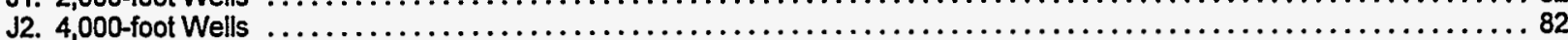

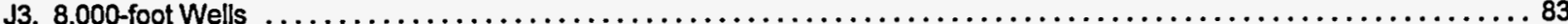

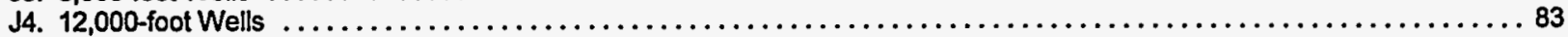

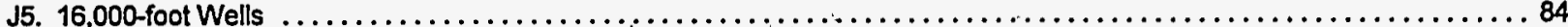

Direct Annual Operating Costs and Indices for Gas Production in South Louisiana

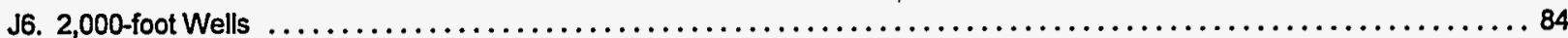

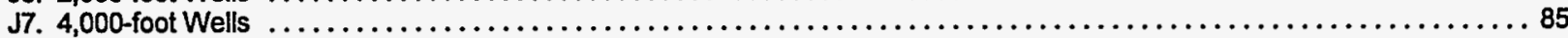

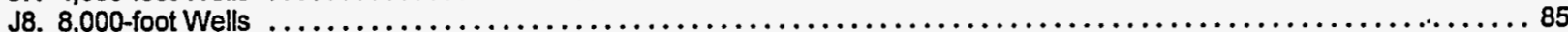

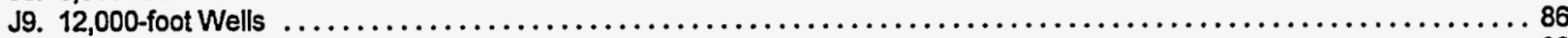

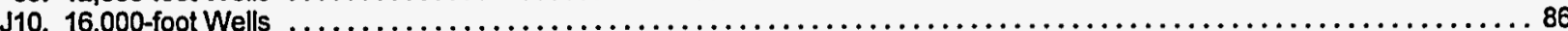

\section{Appendix K}

\section{Lease Equipment Costs and Indices for Gas Production in North Louisiana}

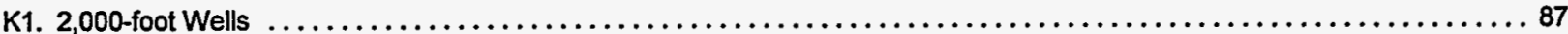

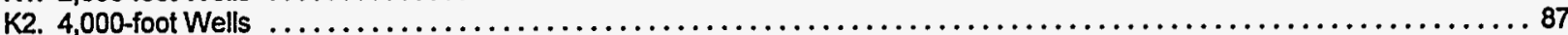

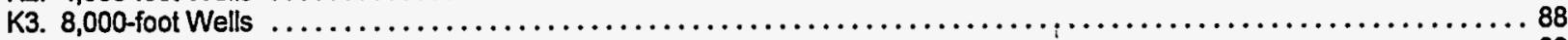

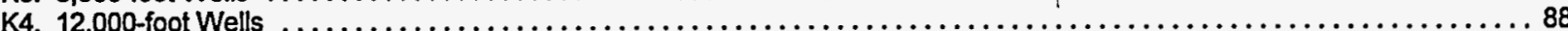

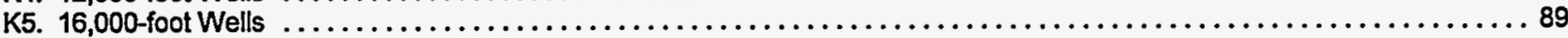

Direct Annual Operating Costs and Indices for Gas Production in North Louisiana

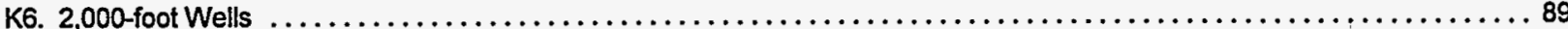

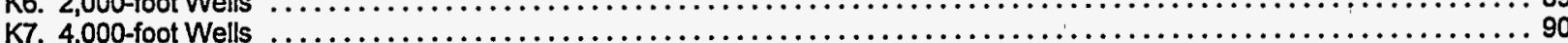

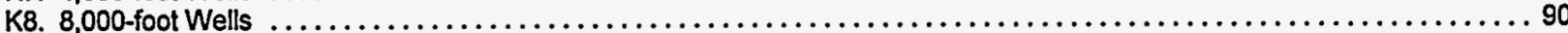

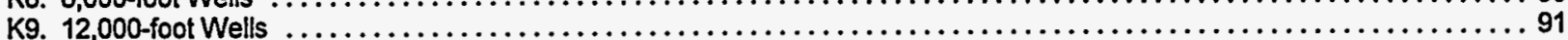

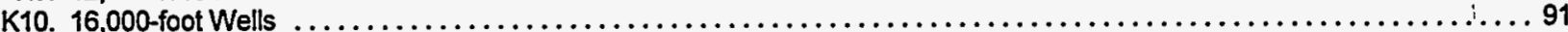

\section{Appendix L}

Lease Equipment Costs and Indices for Gas Production in the Mid-Continent

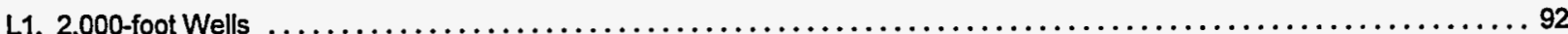

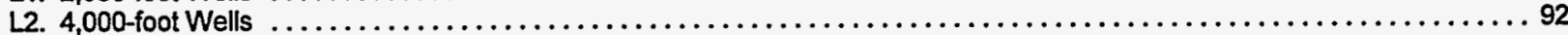

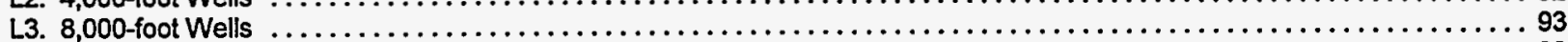

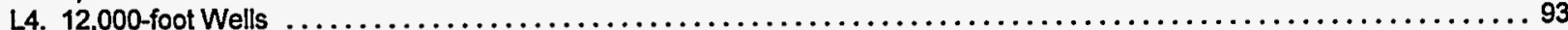

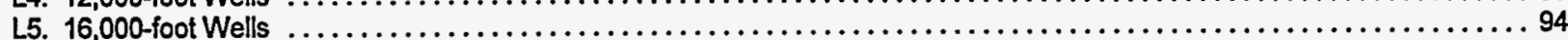

Direct Annual Operating Costs and Indices for Gas Production in the Mid-Continent

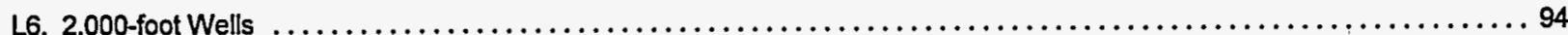

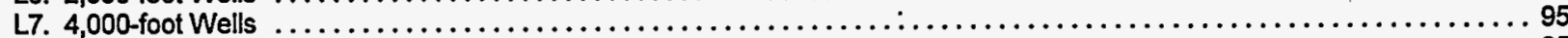

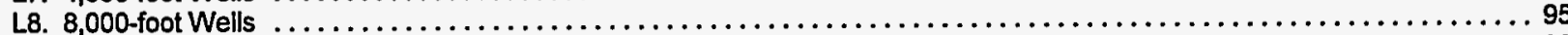

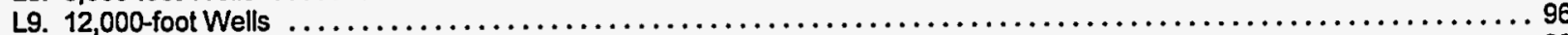

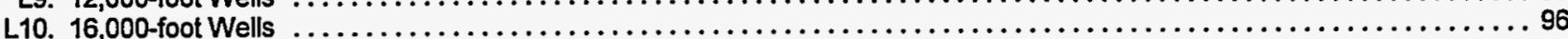




\section{Appendix M}

\section{Lease Equipment Costs and Indices for Gas Production in the Rocky Mountains}

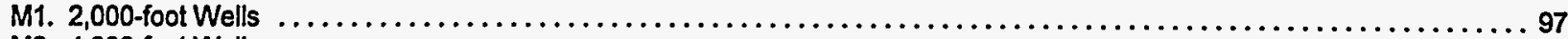

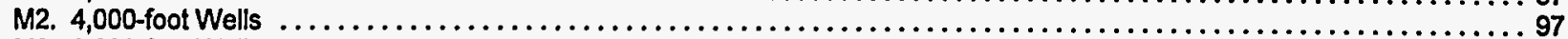

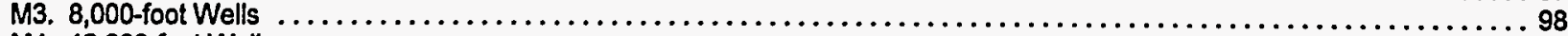

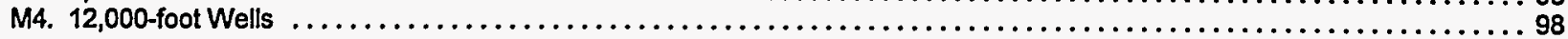

Direct Annual Operating Costs and Indices for Gas Production in the Rocky Mountains

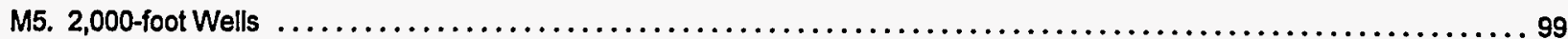

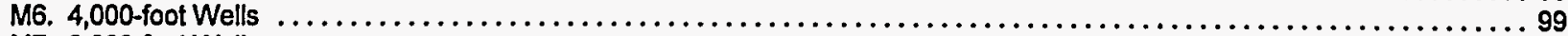

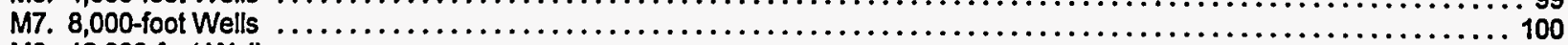

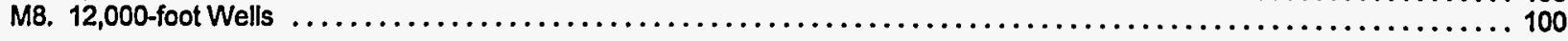

\section{Appendix N}

N1. Indices and Gross Domestic Product Deflated Indices of the Aggregated Average Capital Equipment Costs for Oil and Gas Fields and the Producer Price Index (PPI)

N2. The Gross Domestic Product Implicit Price Deflator and the Gross Domestic Product Deflated Indices of Operating Costs for Oil and Gas Fields 



\section{Executive Summary}

This report presents estimated costs and cost indices for domestic oil and natural gas field equipment and production operations for 1992, 1993, 1994, and 1995. The costs of all equipment and services are those in effect during June of each year. The sums (aggregates) of the costs for representative leases by region, depth, and production rate were averaged and indexed. This provides a general measure of the increased or decreased costs from year to year for lease equipment and operations. These general measures do not capture changes in industry-wide costs exactly because of annual variations in the ratio of the total number of oil wells to the total number of gas wells. The detail provided in this report is unavailable elsewhere. The body of this report contains summary tables, and the appendices contain detailed tables.

Price changes for oil and gas, changes in taxes on oil and gas revenues, and environmental factors (compliance costs and lease availability) have a significant impact on the number and cost of oil and gas wells drilled. These changes also impact the cost of oil and gas equipment and production operations.

Oil and gas prices rose from 1976 to the early 1980's, when deflated oil prices peaked at an index of nearly 260 . In 1976, the average price of oil was $\$ 8.19$ per barrel and the average price of gas was $\$ 0.58$ per thousand cubic feet (Mcf). Deflated gas prices, which also rose to an index of about 260, were at a plateau from 1982 through 1984, before following oil prices downward. The 1995 oil price, after an increase from 1994, represents the second-lowest deflated oil price since 1976. By contrast, deflated gas prices have remained above 1976 prices, although they have headed downward in 1994 and 1995. Clearly, the price trends reflect fundamental differences between the markets for oil and gas.

Figure ES1, with gas prices and operating costs indexed to 1976, shows the differences from 1976 values of deflated gas prices and deflated operating costs for gas wells. The greatest difference between the two series was during 1984, and the current downward trend in product prices, coupled with declining operating costs, point to the fact that producer profitability is much more strongly affected by product prices than by increasing operating efficiency.

Gas activity has been spurred in recent years by favorable tax treatment (including tax credits for tight formations gas and coalbed methane). Environment-related costs for natural gas operations, generally less than for oil operations, may equal those for oil where coalbed methane leases are concerned as the main factor affecting operating costs in some coalbed methane regions is disposal of substantial amounts of formation water produced with the gas. No data have been collected on the environmental costs for gas wells.

Figure ES1. Deflated Natural Gas Price and Operating Cost Indices

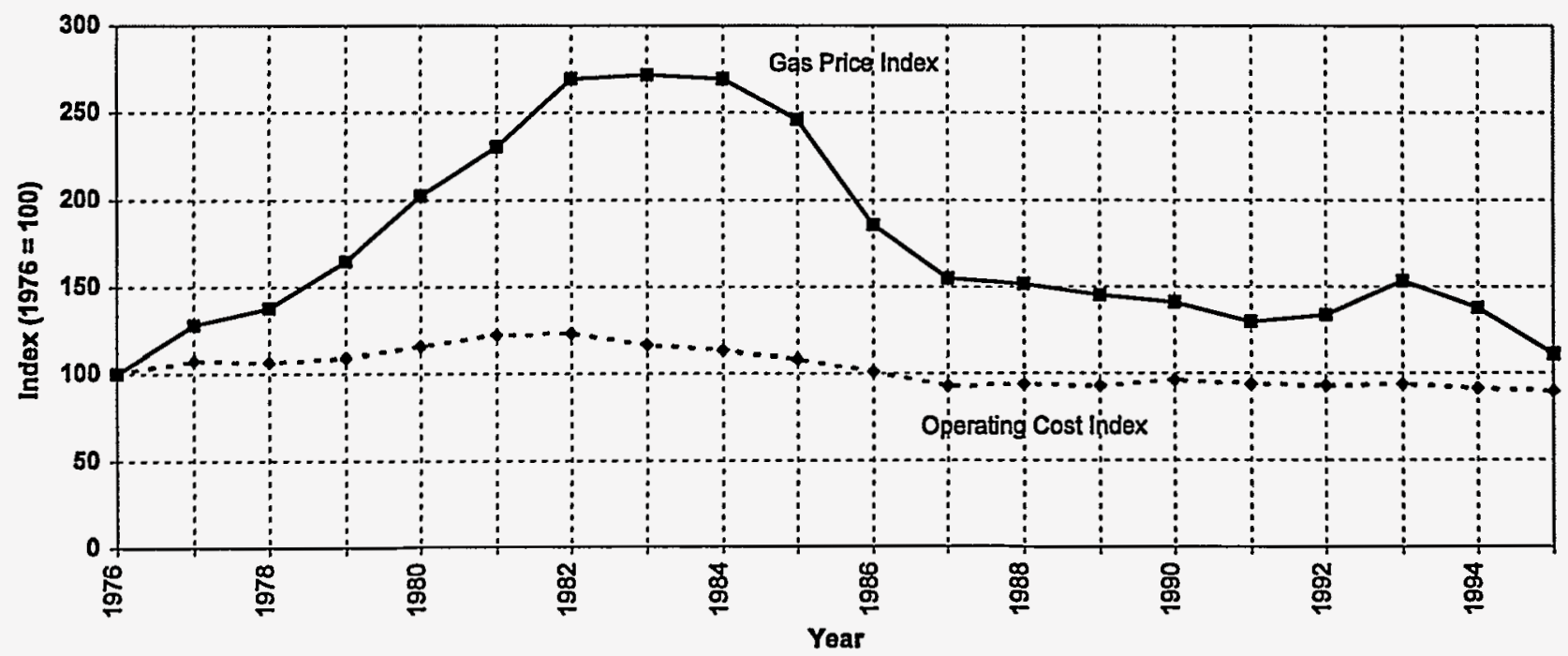

8ourea: Enorgy Information Adminiatration, Ontes of Oll and Gas. 
Figure ES2 similarly depicts deflated oil prices and operating costs indexed to 1976. There are two main differences between Figures ES1 and ES2. First, the gas price index is almost the reverse of that for oil, for oil operating cost index values have remained above 1976 levels throughout while gas operating index values fell below 1976 values in 1986, and have fluctuated within a relatively narrow range since.

Oil operating costs were studied by obtaining equipment and operating costs for representative oil leases for 6 onshore regions of the lower 48 States (see Figure 1). Each lease consists of 10 wells producing by primary means (natural depletion) from depths of $2,000,4,000$, 8,000 , and 12,000 feet. The aggregate average lease equipment costs for the six regions and four depths dropped slightly from 1992 to 1995 . Since 1992, nontubing costs have risen more than tubing prices.

Oil production is an energy intensive operation, and when fuel prices (natural gas prices) increase, so do oil production costs. Gas production is more labor intensive with only minor fuel costs. Therefore, high energy prices are a boon to gas producers and the natural gas producing industry has fared better than the oil producing industry for the past decade. The change in gas prices has surpassed the change in gas well operating costs. Oil prices have fallen faster and farther than oil field operating costs since 1981, narrowing the profit margin and reducing the amount of internally raised capital available for investment in drilling and production operations. Costs and indices for additional waterflood oil recovery equipment and its operation were calculated for leases with well depths of $2,000,4,000$, and 8,000 feet in west Texas. Cost differentials between primary and secondary and primary operations in this region are presumed to be similar to those in other areas.

The aggregate average additional equipment cost for secondary recovery (waterflood) in 1995 was about 3 percent less than in 1992. The large 1994 cost drop in these costs was primarily due to decreases in drilling costs. Waterflood operating costs followed the direction of those of primary oil recovery in west Texas, showing an increase of about 6 percent from 1992 to 1995.

Useful insights from the data in this report lie primarily in the differences that are presented. The costs for equipment and operations are different in each area, differ between primary and secondary operations and differ between gas and oil operations. Cost trends for some items vary widely from time to time, while others remain unchanged for years at a time. The summary tables in Chapter 3, Discussion of Results, indicate the overall trends while the appendix tables provide the detail needed by many readers.

\section{Figure ES2. Deflated Oll Price and Operating Cost Indices}

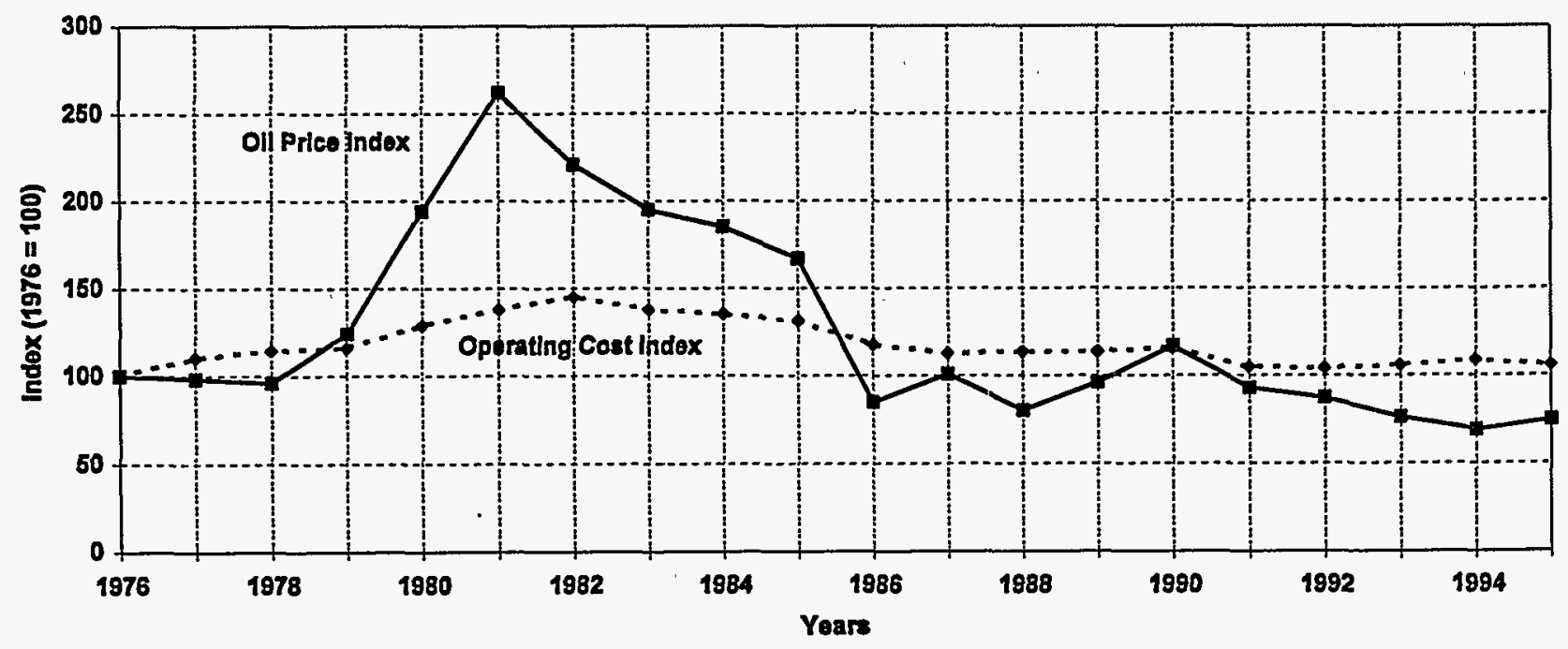

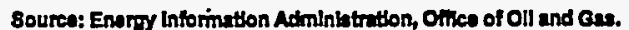




\section{Introduction}

This report marks the continuation of the EIA series on equipment and operating costs and cost indices for oil and gas leases. In addition to cost comparisons within the petroleum industry, the data reported here are often used to assess the economic effects of specific plans and policies relating to the industry. No other source offers the detail of this report.

Only a few publications contain or have contained data relating to costs in the petroleum industry. For several years, the American Petroleum Institute (API), the Independent Petroleum Association of America, (IPAA), and the Mid-Continent Oil and Gas Association have published cost statistics in their Joint Association Survey of the U.S. Oil and Gas Producing Industry 1,2. Section I of that publication pertains to drilling costs. Section II (discontinued after 1975) presented total U.S. expenditures for exploration, development, and production.

For several years the U.S. Bureau of the Census surveyed a group of companies classified by size and published a report titled Annual Survey of Oil and Gas ${ }^{3}$ in its Current Industrial Report series. These reports contained oil and gas operating cost data for both direct and indirect expenses but were discontinued after 1982 .

Other than EIA's, no series of non-aggregated oil and gas field equipment and operating costs and subsequent indices has been published on a regional basis. Equipment and operating costs vary from region to region because of differences in fuel costs, labor rates, and other variables. (See Figures 1 and 2 for areas of oil and gas production.) Therefore, equipment and operating costs and cost indices are estimated by EIA on a regional basis for both oil and gas fields.

The costs and cost indices provided in this report are for representative lease operations with equipment and operating procedures designed by EIA staff engineers for representative 10-well oil leases producing by artificial lift or 1 flowing well per gas lease. The design criteria took into account the predominant methods of operation in each region. Individual items of equipment were priced by using price lists and by communication with the manufacturer or supplier of the item in each region. Except as mentioned in the Executive Summary and treated in Appendix N, all costs presented in this report are current to their year and not adjusted for inflation.
Freight costs and installation costs were determined based on regional rates. These costs were summed for each category of equipment. For example, the category listed as "pumping equipment" for a rod pump system includes:

- A pumping unit

- Additional counter-weights

- Crank guards

- Belt guards, V-belts and sheaves

- Freight costs

- Installation costs.

Conversion of primary oil producing leases to secondary recovery (waterflood or water injection) involves:

- the drilling and equipping of 11 water injection wells

- the installation of water supply, storage, treatment, high pressure injection equipment and related piping

- replacement of production facilities with larger equipment.

Costs for gas activities were investigated by determining equipping and operating costs for representative gas leases producing from depths of $2,000,4,000,8,000,12,000$ and 16,000 feet in 6 onshore regions of the lower 48 States. The summary tables contain composite costs and indices for flow rates of 50,250,500,1,000,5,000, and 10,000 thousand cubic feet (Mcf) of gas per day by depth and region.

Equipment for gas wells does not cover hydrogen sulfide removal, compression, or special equipment for water removal. Tubing is also not included in the equipment list for gas wells.

'American Petroleum Institute, Independent Petroleum Association of America, Mid-Continent Oil and Gas Association, 1994 Joint Association Survey on Drilling Costs. (Washington, DC, November 1995), American Petroleum Institute.

\footnotetext{
${ }^{2}$ American Petroleum Institute, Independent Petroleum Association of America, Mid-Continent Oil and Gas Association, Joint Association Survey of the U.S. Oil and Gas Producing Industry, 1974, Section II: Expenditures for Exploration, Development and Production, (Washington, DC, May 1976), American Petroleum Institute.

${ }^{3}$ U.S. Bureau of the Census, Annual Survey of Oil and Gas, 1981, Current Industrial Reports pub. MA-13K (81)-1, (Washington, DC, March 1983).
} 
Figure 1. Geographical Regions for Oil Producing Leases

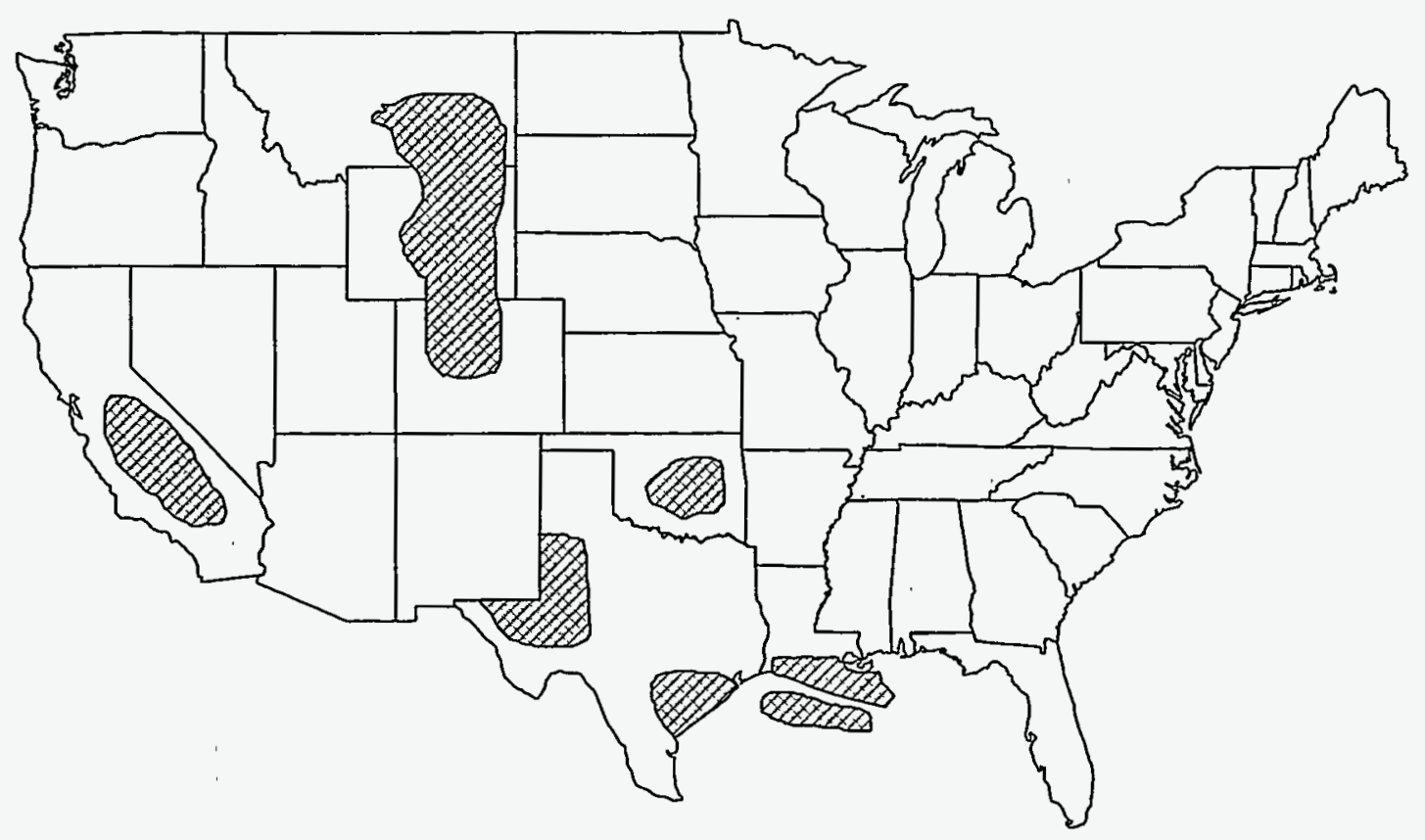

Source: Energy Information Adminlstration, Office of Oil and Gas.

Figure 2. Geographical Regions for Gas Producing Leases

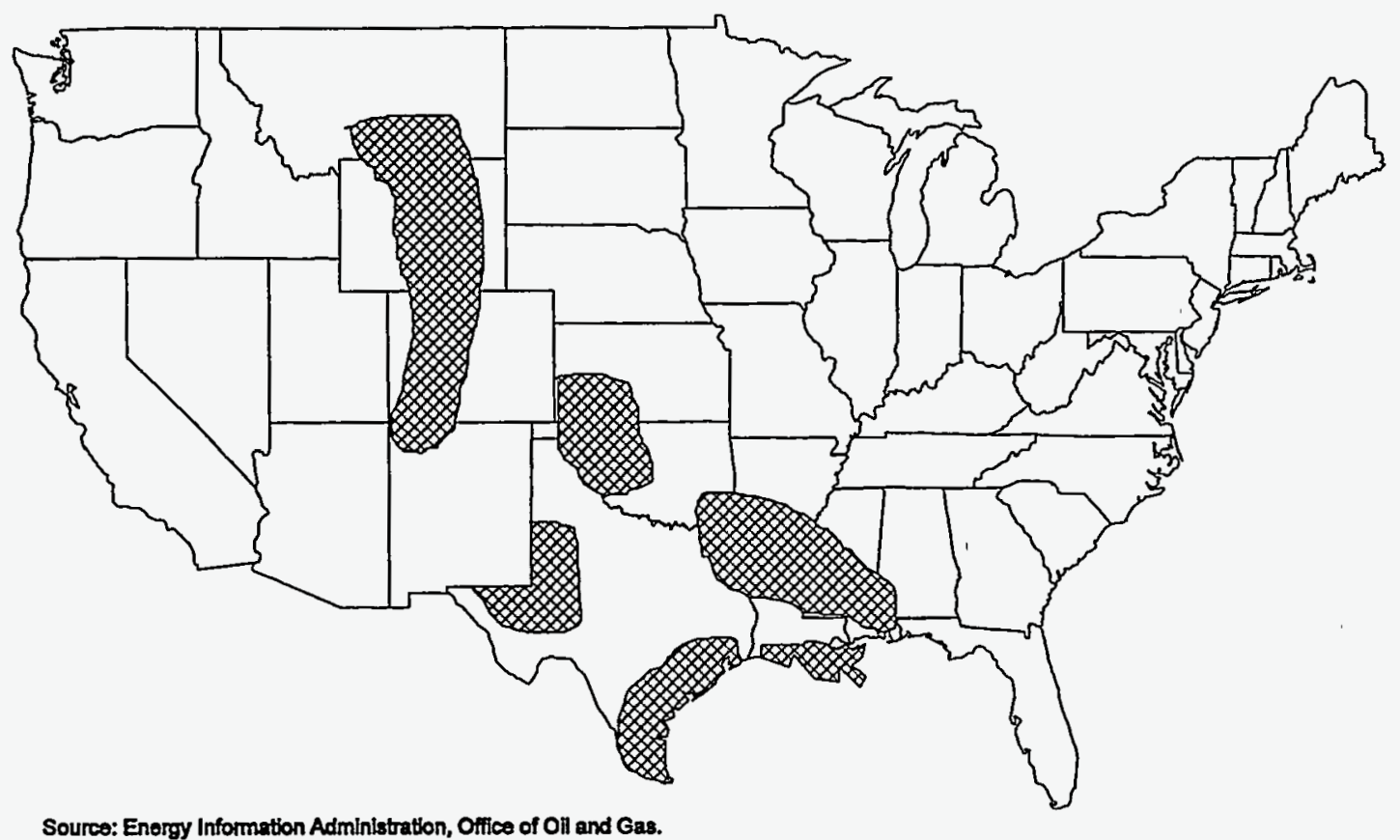




\section{Indexing Procedure}

The leading supply, service, and contracting companies (active in one or more of the regions) were contacted every year (1976 through 1995) for local June prices for their component of equipment or operating function. The objective of this process was to acquire prices that were representative for each region. Total component costs were determined from these prices and summed to permit indexing.

The indices in this report reflect changes in the costs of items, and their related transportation charges and associated installation costs on representative leases. The index numbers in this report are "pure" cost indices. A pure cost index measures the change in cost of a fixed quantity of goods and/or services. Pure cost indices are applied to the individual line items presented in the appendix tables.

The subtotal and total indices are composite indices. A composite index measures the change in cost of an aggregate of goods and/or services. Any equipment changes that may be made due to technological advances will be reflected in the composite indices.

The annual operating cost indices measure the change in direct costs incident to the production of oil and gas and exclude changes in indirect costs such as depreciation, and $a d$ valorem and severance taxes.
The indices are calculated with 1976 as the base year as follows:

1995 index $=(1995$ costs $/ 1976$ costs $) \times 100$.

Annual percentage changes can be determined by dividing the last year's index by the prior year's index, subtracting one (1.0), and multiplying by 100 . For example, to find the percent change from 1994 to 1995 , divide the 1995 index by the 1994 index, subtract one (1.0), and multiply by 100 .

The estimated region equipment costs for the representative oil leases were summed, averaged, and indexed by depth, providing a general measure of equipment expenditures relative to depth. The estimated region equipment costs for the representative gas leases were also summed, averaged, and indexed by depth and by production rate. The aggregate average cost for all regions and depths were indexed to allow general trend analyses by year. This same procedure was applied to the annual operating costs for the formulated oil leases and for the formulated gas leases. 


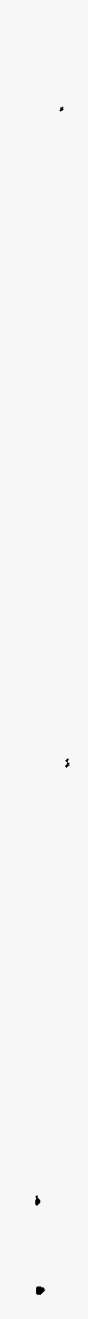




\section{Discussion of Results}

The summary of composite data and the detailed appendix tables permit analysis of equipment and operating costs for each region, depth, method, and type of production. The data in this report should be considered as revised, except for the 1995 data, which are preliminary. Some of the revisions which appear in this report affected equipment costs for the entire series, beginning in 1976. Though these were small, in some cases, the equipment cost revisions reflected a minor change in operating costs. There were no major revisions. The following is a discussion of the composite costs and indices.

\section{Overview}

This report continues a data series begun in 1976, providing a history of equipment and operating costs for oil and gas leases from 1976 through 1995. (See Appendix $\mathrm{N}$ for more detailed information).

Figure 3 shows indices of the aggregate average costs for oil well equipment and operations, indicating general upward pressure on costs. The period of rapid cost increase which began before 1976 changed in 1982, the peak year for total equipment costs, which was followed by prices rising and falling within a range somewhat near the 1982 level. Operating costs followed a different path. The post-1982 drop was minimal, and the 1982 level has been exceeded eight times. Operating costs for 1995, very near the all-time peak set in 1994, have been largely influenced by energy costs (natural gas and electric power) and the costs of oil field services such as well servicing units and chemicals.

Figure 4 is a plot of tubing costs for 10-well oil leases. The type of tubing used for deeper wells not only costs more than that used for shallower wells, but price variations have been more extreme. Prices for 12,000-foot wells dropped in 1994 and rose in 1995. Costs for shallower wells followed the same trend.

Figure 5 is a plot of oil lease equipment costs excluding tubing. Contrasting Figures 4 and 5 , the non-tubing equipment costs vary much less than those for tubing. However, the 1982 non-tubing equipment costs were nearly double the 1976 costs. Non-tubing equipment costs declined from 1982 through 1986 and have generally increased since.

The 1995 index of non-tubing equipment cost for all depths is about 190, which is 90 percent higher than the

Figure 3. Aggregate Average Cost Indlces for Primary Oll Recovery, 1976-1995 (Operation and Equipment, With and Without Tubing Costs)

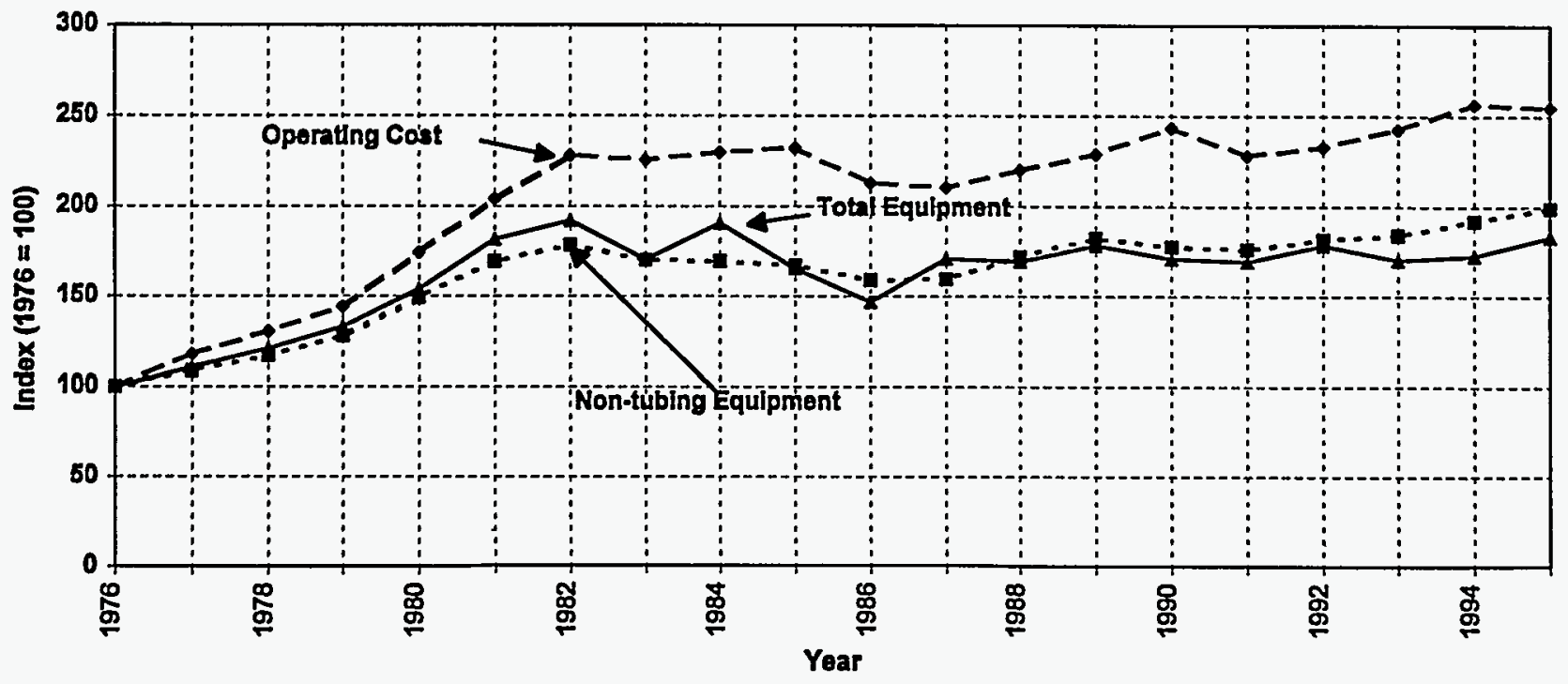

sourcs: Enomy Information Administration, Ortes of Oll and Gas. 
1976 cost. Since 1976, the non-tubing cost for 8,000-foot wells was exceeded by that for 12,000-foot wells about half of the time, an anomaly related to the mixture of pumping equipment types used for 8,000-foot wells.

The availability of well service units (WSUs) is widely used as an indicator of price pressures on operating costs. When WSU utilization is high, prices of other operating cost items are usually firm. The active WSU count rose from about 2,600 in 1976 to 4,850 in 1981, when activity levels peaked (see Figure 6). Although the 1992 active WSU count dropped to near 1976 levels, later counts show a modest increase. Pressure on the well service industry was the result of overbuilding in the early 1980's. With a peak of about 8,000 WSU's available in 1985 , the portion of WSUs at work was less than 60 percent. In 1986, working WSUs were only 40 percent of those available, and 1995 surveys reflect that 62 percent of the WSUs were working. Industry reports show that there are labor constraints that may limit the level to which active WSU's might rise without substantial increases in cost for the operators.

Figure 7 contains the equipping and operating cost indices for gas wells (note that gas well equipment costs do not include tubing costs). The index for gas equipment costs increased steadily from 1976 to a peak of about 183 in 1982. Lower levels of activity forced the index to a low of 153 in 1986, from which time costs increased to set new highs between 1990 and 1993, with a slight drop to 1995. The 1992-1995 drop in equipment costs was 4 percent. Operating costs have set new highs beginning with 1990, although there was a slight drop in 1995. Recent downward movement in equipment prices from a high in 1993 seems to be based on higher levels of competition, nationally and internationally. Operating costs rose at a steadier pace than equipment costs because of recent changes in labor prices, which are a major influence on the overall costs of gas well operation. The use of gas for fuel on gas leases is relatively insignificant, so changing gas prices had little effect.

\section{Equipment Costs for Oil Leases}

\section{Primary Recovery}

Table 1 is a summary of the composite lease equipment costs and indices for primary oil recovery operations in 6 onshore producing regions by depth. The trends in costs varied by depth and region. The aggregate (or sum) of the 10-well oil lease equipment costs for the six regions and 4 depths decreased by less than 1 percent in the period from 1992 to 1995 , although there were noticeable drops in both 1993 and 1994. Table 1 also presents the average costs and indices of the 6 regions by depth. As shown in Figure 8, the average equipment costs increase with depth.

In this figure, the variations by depth by year present slightly dissimilar patterns, as, for example, aggregate costs for 8,000- and 12,000-foot wells in 1993 and 1994 did not follow expected trends of increasing costs with time and depth, due primarily to lower costs for tubular goods (see Figure 4). Although there are regional differences in equipment costs for each depth of wells, the range of indexed values is larger foroperating costs. The significant fact is that small negative annual price changes from 1992 through 1994 changed to a 6 percent increase in the aggregate average index for 1995.

\section{Secondary Recovery}

Table 2 summarizes the additional lease equipment costs and indices associated with secondary oil recovery (waterflood) from depths of 2,000, 4,000, and 8,000 feet in west Texas. This region was the focus of a substantial part of the early secondary recovery work in the country, and the differences between primary and secondary costs are presumed to be similar to those in other regions. The method used in this report is waterflooding. The additional lease equipment is the equipment needed to convert from a primary recovery operation to a secondary recovery operation. The aggregate decrease in additional equipment costs was almost 1 percent for the 1992-1995 period. As noted before, drilling cost estimates are subject to major annual revisions and, as drilling costs can account for more than one-half of the additional equipment costs, revisions to drilling costs may obscure the changes in other costs. During the 1992-1995 period, however, changes in drilling costs did not follow those of nondrilling costs, for they dropped about 9 percent while other prices remained essentially static. Figure 9 shows the additional costs of waterflood equipment for depths of 2,000, 4,000, and 8,000 feet for 1992 through 1995. Insurance costs for platform and production equipment

\section{Operating Costs for Oil Leases}

\section{Primary Recovery}

Table 3 is a summary of the annual operating costs and indices for primary oil recovery operations which are shown in Figure 10 while those for secondary operations are shown in Figure 11. The average for the aggregate of the operating costs for the 6 regions and 4 depths was $\$ 209,400$ for the 10 -well lease in 1995 . This represents 
Figure 4. Tubing Costs for Oil Leases, 1976-1995 (10 Producing Wells)

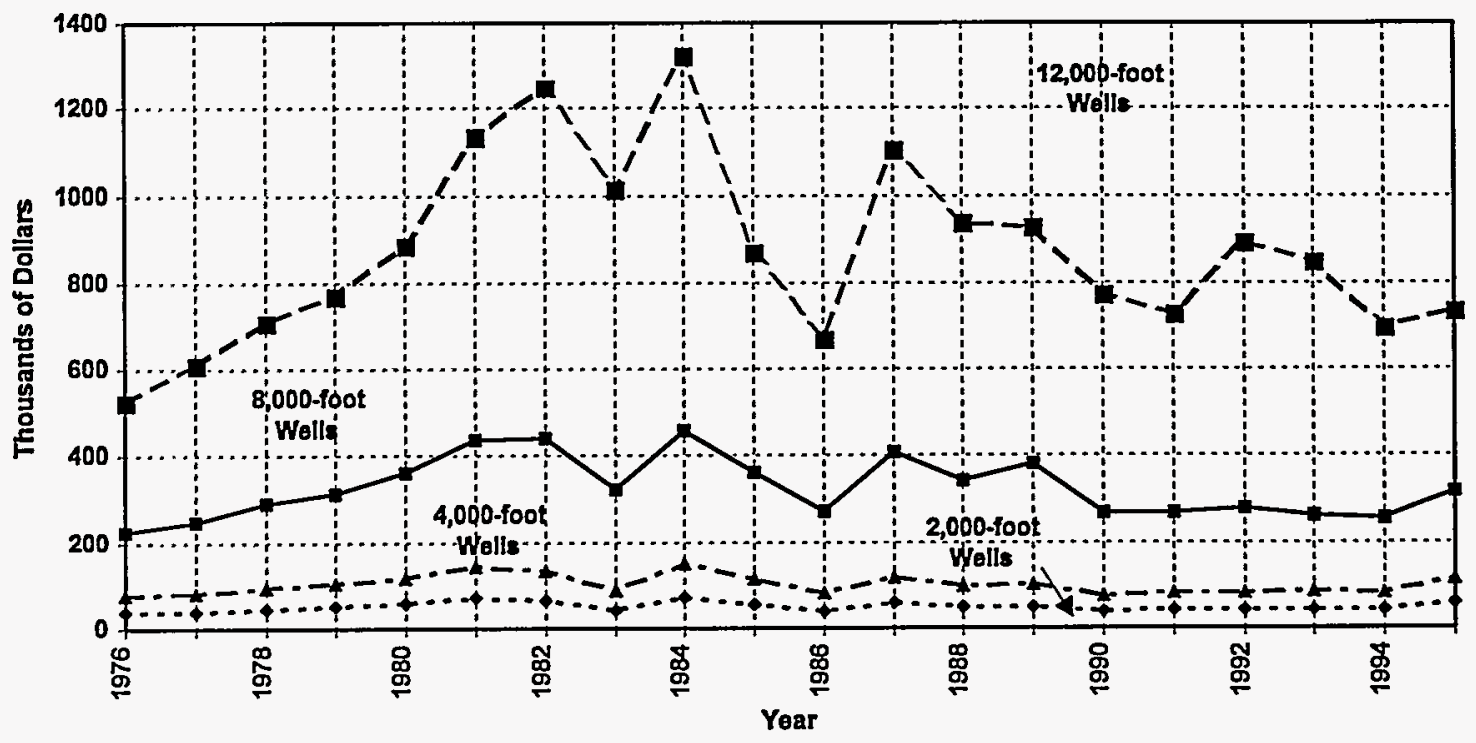

Source: Enargy Information AdmInistration, Office of Oll and Gas.

Figure 5. Non-tubing Equipment Costs for Oil Leases, 1976-1995 (10 Producing Wells)

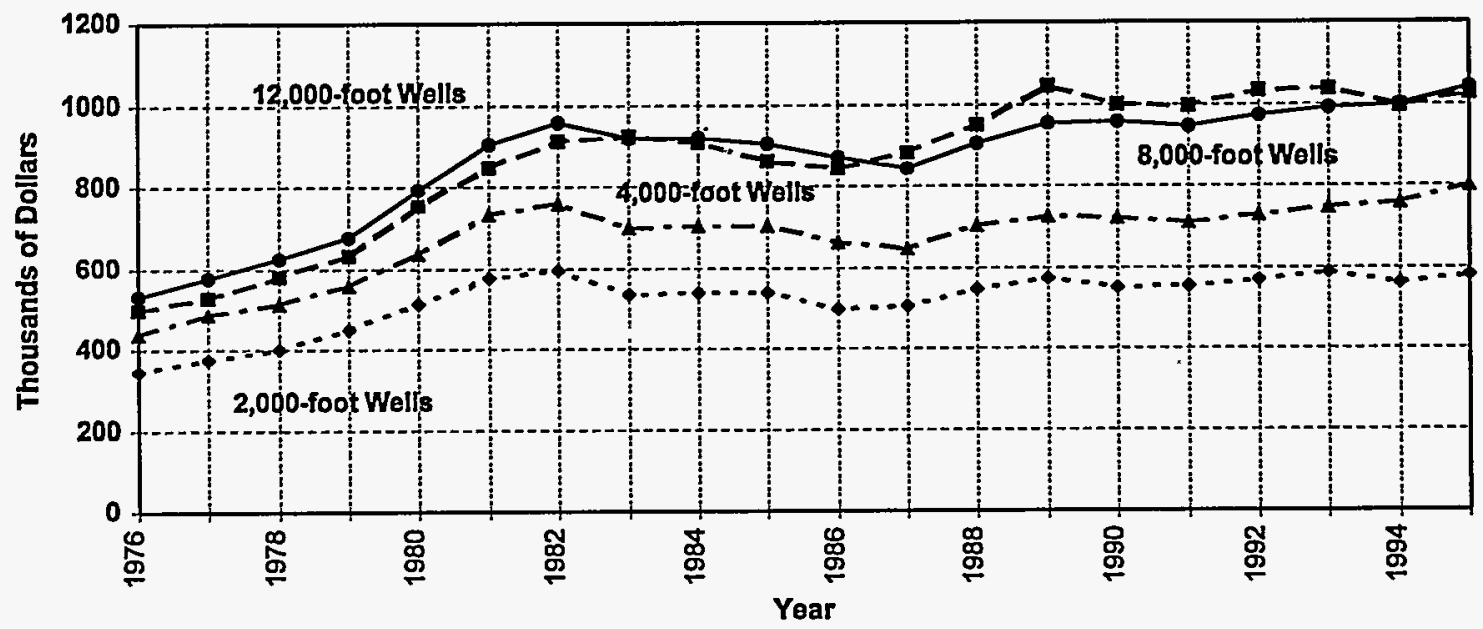

8oures: Energy Information Adminiatration, Office of Oll and Gas. 
Figure 6. Well Service Units, 1976-1995

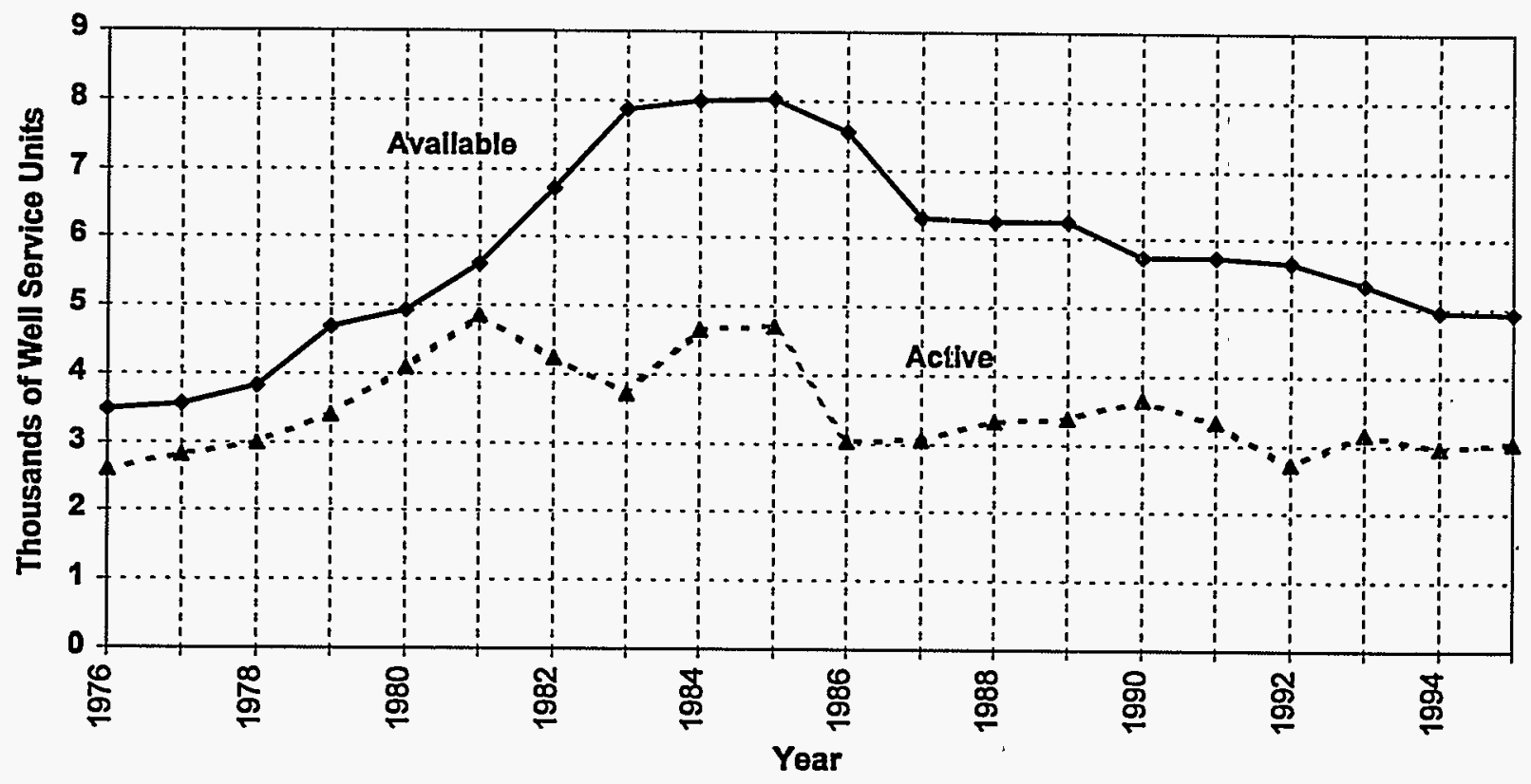

Source: Enorgy Information Adminlatration, Office of Oll and Gas.

Figure 7. Aggregate Average Cost Indices for Gas Recovery, 1976-1995 (Operation and Non-tubing Equipment Costs)

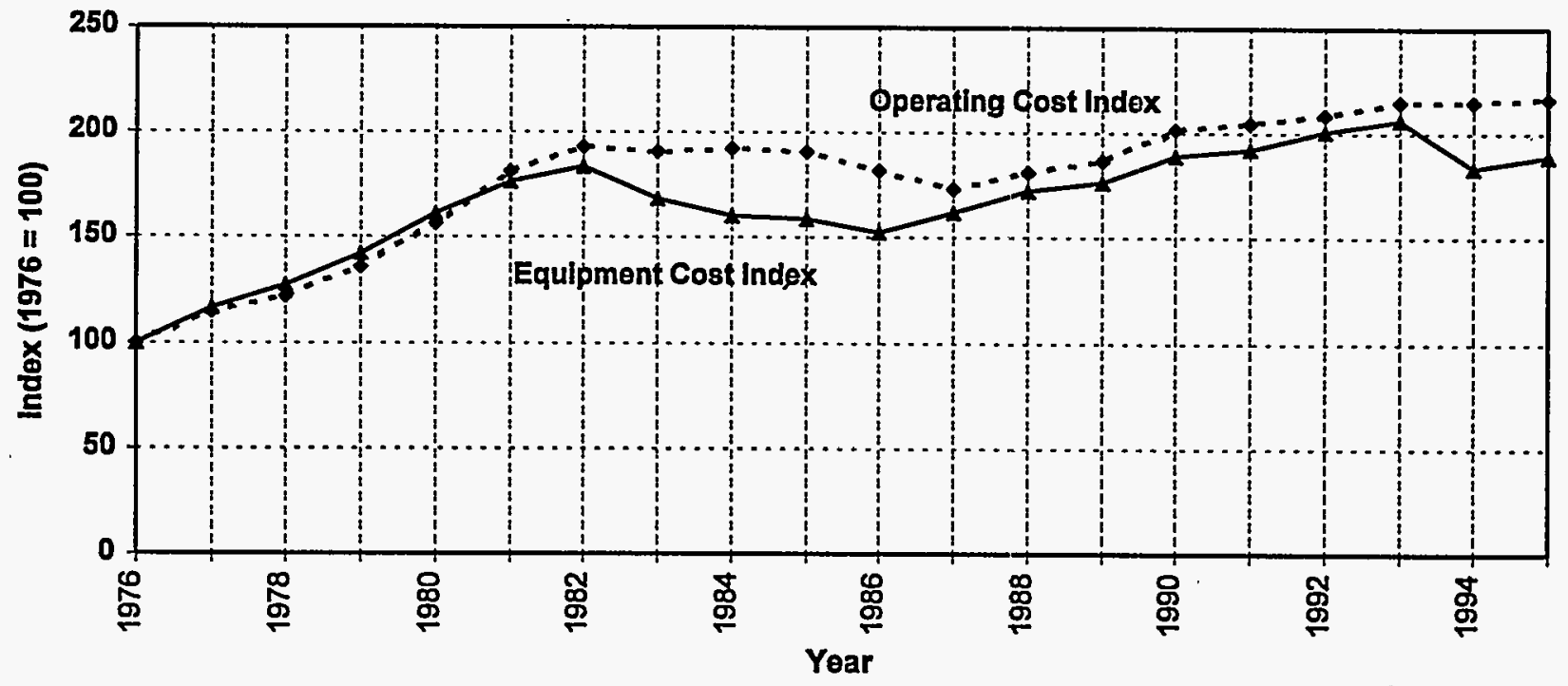

Source: Enorgy Information Administration, Ofice of Oll and Gas. 
Table 1. Summary of Lease Equipment Costs and Composite Indices for Primary Oil Recovery Operations (10 Producing Wells)

\begin{tabular}{|c|c|c|c|c|c|}
\hline \multirow[b]{2}{*}{ Area } & \multicolumn{4}{|c|}{ Index $(1976=100)$} & \multirow{2}{*}{$\begin{array}{c}\text { 1995 } \\
\text { Cost } \\
\text { (dollars) }\end{array}$} \\
\hline & 1992 & 1993 & 1994 & 1995 & \\
\hline & \multicolumn{5}{|c|}{ 2,000-Foot Wells } \\
\hline 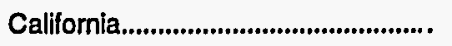 & 161.7 & 164.7 & 166.7 & 177.6 & 891,600 \\
\hline 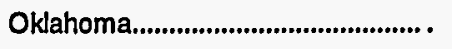 & 263.5 & 272.1 & 276.3 & 293.9 & 647,700 \\
\hline South Louisiana................................... & 266.0 & 273.0 & 280.7 & 297.8 & 711,400 \\
\hline 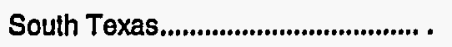 & 269.6 & 281.0 & 285.8 & 303.5 & 676,700 \\
\hline 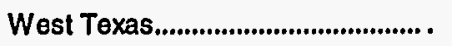 & 168.2 & 172.9 & 175.5 & 186.4 & 657,900 \\
\hline Rocky Mountains................................... & 169.3 & 173.3 & 174.5 & 184.8 & 641,200 \\
\hline \multirow[t]{2}{*}{ Average or Index } & 202.3 & 207.9 & 211.2 & 224.3 & 704,400 \\
\hline & \multicolumn{5}{|c|}{ 4,000-Foot Wells } \\
\hline 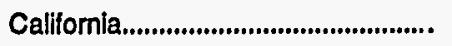 & 141.9 & 144.5 & 146.7 & 158.0 & $1,109,700$ \\
\hline 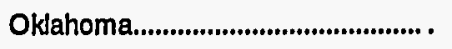 & 207.4 & 215.2 & 217.9 & 240.3 & 896,600 \\
\hline South Louisiana.................................... & 183.3 & 185.5 & 192.0 & 204.2 & 869,800 \\
\hline South Texas......................................... . & 171.7 & 176.5 & 182.0 & 192.7 & 839,600 \\
\hline West Texas........................................... & 153.1 & 158.2 & 160.2 & 176.5 & 897,600 \\
\hline Rocky Mountains................................ . & 151.7 & 155.2 & 156.2 & 172.4 & 866,000 \\
\hline \multirow[t]{2}{*}{ 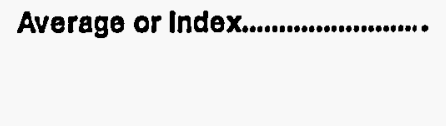 } & 164.2 & 168.3 & 171.4 & 185.9 & 913,200 \\
\hline & \multicolumn{5}{|c|}{ 8,000-Foot Wells } \\
\hline 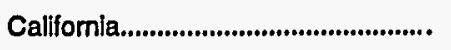 & 170.3 & 165.6 & 165.9 & 171.8 & $1,568,200$ \\
\hline 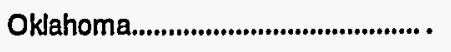 & 202.8 & 172.0 & 175.1 & 190.5 & $1,395,900$ \\
\hline South Louisiana..................................... & 178.6 & 180.5 & 188.0 & 204.2 & $1,125,000$ \\
\hline 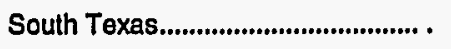 & 164.1 & 168.6 & 175.0 & 189.3 & $1,068,800$ \\
\hline 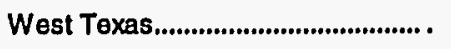 & 146.0 & 151.6 & 156.9 & 173.7 & $1,540,700$ \\
\hline 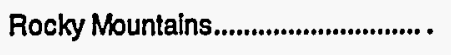 & 141.2 & 144.1 & 145.7 & 159.9 & $1,427,100$ \\
\hline \multirow[t]{2}{*}{ Average or Index............................ } & 165.3 & 161.9 & 165.5 & 179.0 & $1,354,300$ \\
\hline & \multicolumn{5}{|c|}{ 12,000-Foot Wells } \\
\hline 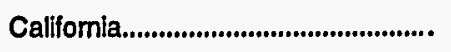 & 196.1 & 174.4 & 163.3 & 169.6 & $1,909,800$ \\
\hline 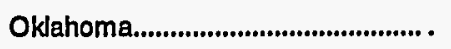 & 201.0 & 175.6 & 163.7 & 170.4 & $1,655,000$ \\
\hline 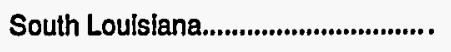 & 202.5 & 178.5 & 169.1 & 176.3 & $1,820,100$ \\
\hline South Texas............................................. . & 200.8 & 178.4 & 168.1 & 174.4 & $1,747,000$ \\
\hline West Texas............................................ & 202.0 & 176.3 & 164.7 & 171.1 & $1,700,900$ \\
\hline Rocky Mountains..................................... & 206.1 & 180.8 & 168.6 & 175.4 & $1,703,900$ \\
\hline Average or Index........................... & 201.3 & 177.3 & 166.2 & 172.8 & $1,756,100$ \\
\hline Aggregate Average.......................... & 183.8 & 174.7 & 172.4 & 183.4 & $1,182,000$ \\
\hline
\end{tabular}

- Pralliminary

Note: Reported average or aggregate average indices are indices of the average costs. They are not an average of the index values.

Source: Energy Information Administration, Office of Oll and Gas 
Figure 8. Aggregate Average Lease Equipment Costs for Primary Oil Recovery, 1992-1995 (10 Producing Wells)

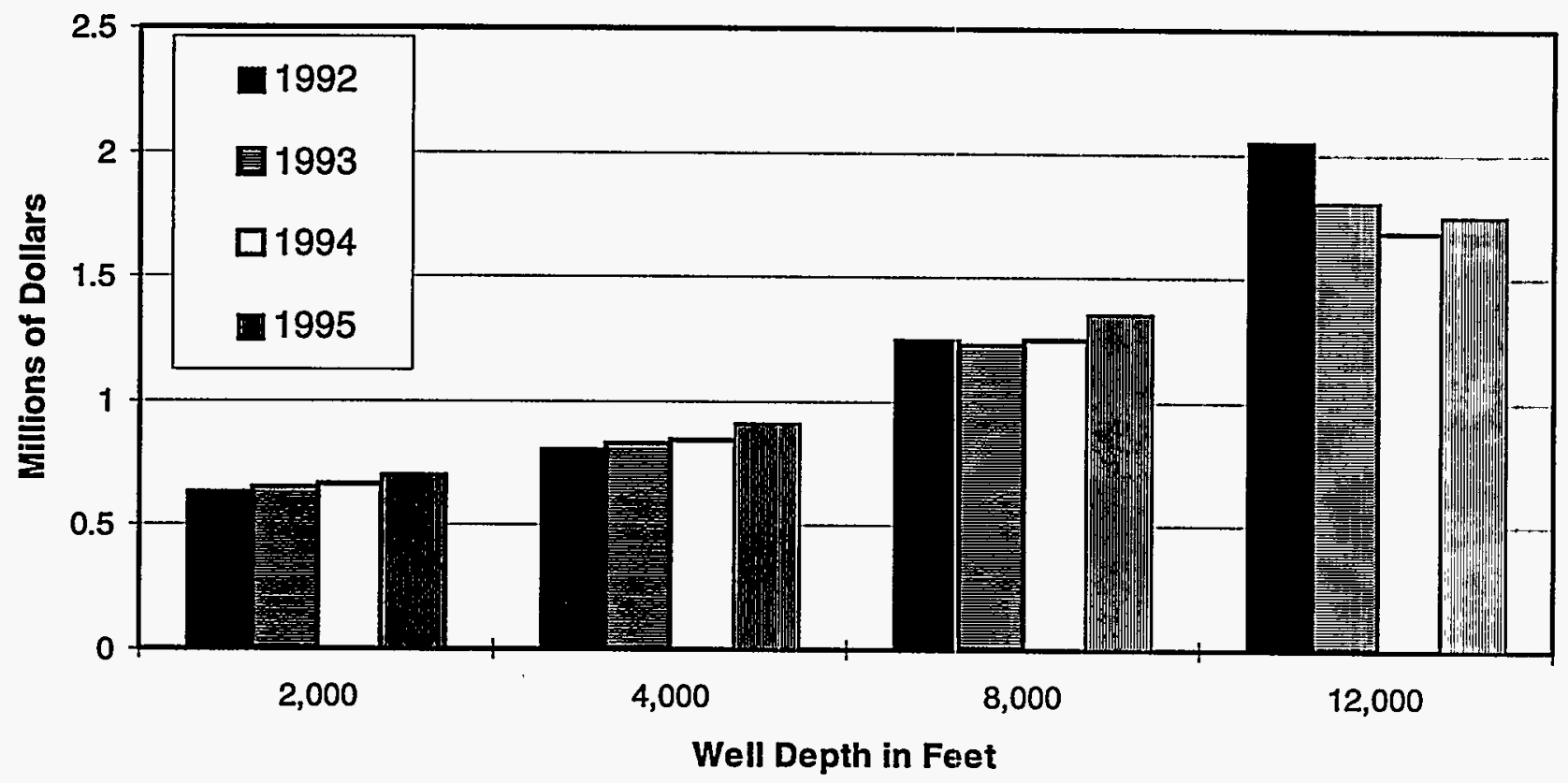

Source: Table 1

Figure 9. Additional Cost of Lease Equipment for Secondary Oil Recovery in West Texas, 1992-1995 (10 Producing and 11 Injection Wells)

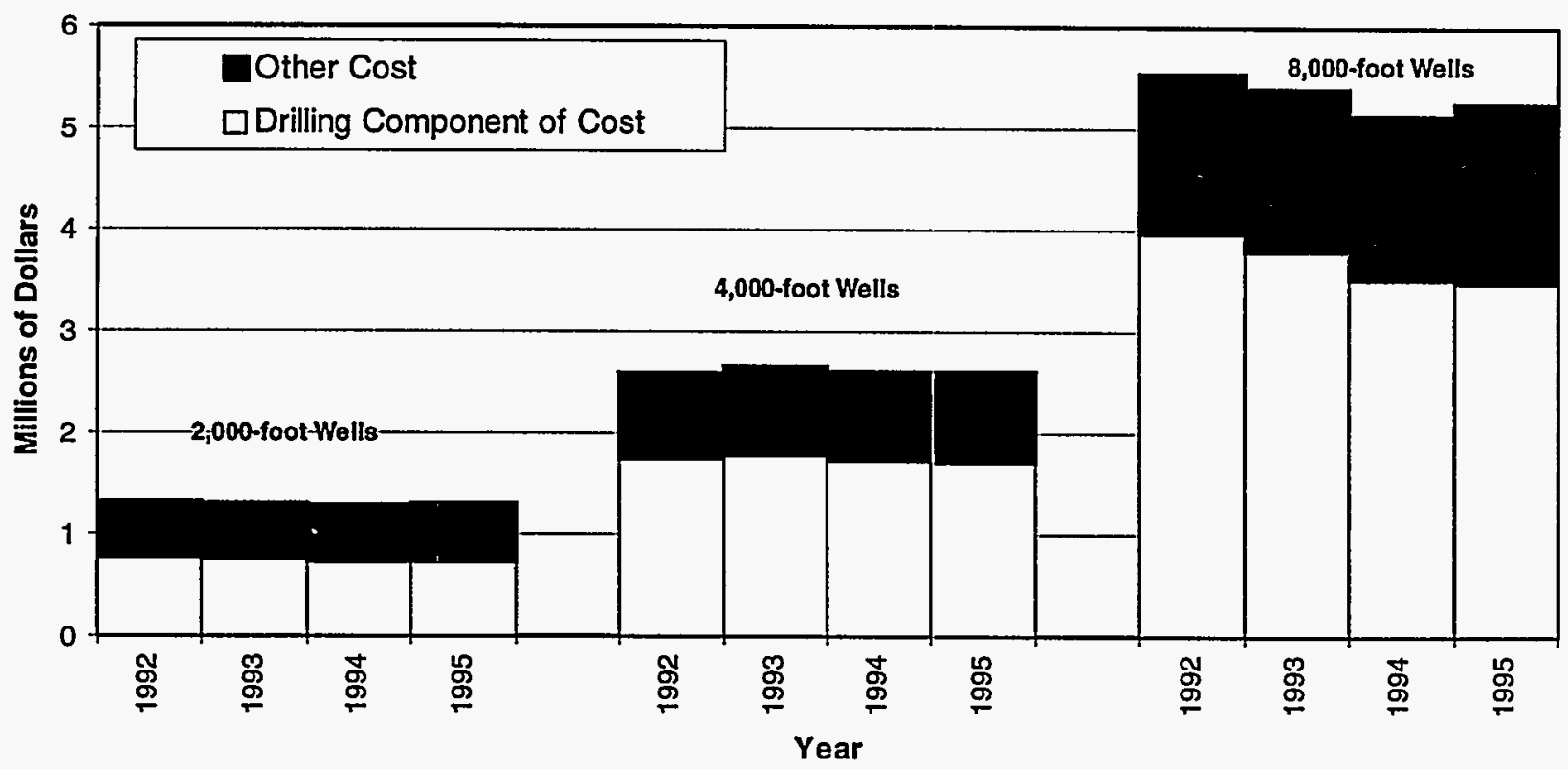

Source: Table 2 
Table 2. Summary of Additional Costs and Composite Indices for Lease Equipment and Injection Wells In West Texas for Secondary Oll Recovery

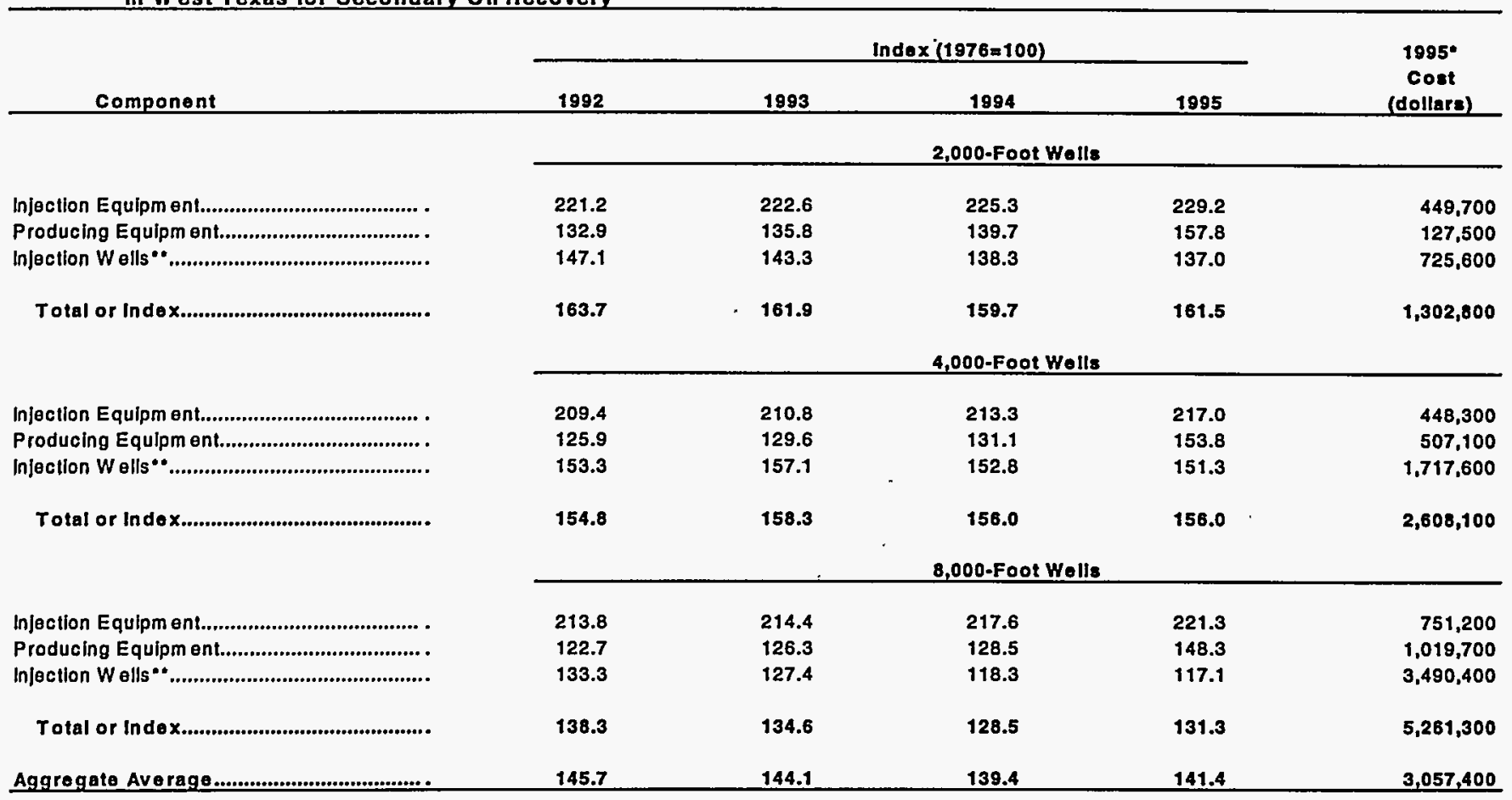

- Proliminary

"Costs from Joint Assoclation Survey data.

Note: Reported average or aggregato average indices are indices of the average costs. They are not an average of the Index values.

Source: Energy Inform ation Adm Inistration, Ofice of Oll and Gas

about a 5 percent increase over the 1992-1995 period. Examination of Table 3 shows that cost trends for oil operations varied widely from 1992 to 1995 . Indexed costs for 1994 were generally flat compared to those for 1993 for 2,000- and 4,000-foot wells, 8 percent more for 8,000-foot wells, and 14 percent more for 12,000-foot wells. The 1976 to 1995 history of aggregate operating costs is shown in Figure 3. The index for 1994 replaced the index of 1990 as the peak year since 1976 . There was negligible change in 1995.

Changes in individual componenits of operating costs show large variations. Fuel, power and water costs comprise one of the most volatile components for oil leases, primarily due to changes in the average price of natural gas in the different regions. Overall costs for fuel, power and water ranged from an increase of about 12 percent for the 1992-1995 period for south Louisiana to an 18 percent decrease for Oklahoma. Contrasted with the rest of the nation, the primary energy source for the California and Rocky Mountain regions is electricity. In the Rocky Mountains, electricity is generated by coal-fired plants and hydroelectric plants that have adequate water supplies. In California, hydroelectric plants operate at full generating capacity as long as there is an ample water supply. However, their water supply is weatherdependent with little or no reserve, so a dry winter can cause reduction in hydroelectric generation of electricity the following year. The reduction in hydroelectric capacity is made up by power from generators using alternate fuels, or is purchased from out-of-state sources. Therefore, electric rates can vary widely in California. Figure 12 depicts fuel, power, and water indices for 12,000-foot wells in the Rocky Mountains and California. Note that the fuel, power and water costs decreased from 1985 to 1991 for the Rocky Mountains, followed by a small increase in 1992, then a 3 percent increase in 1993. Costs in 1994 dropped by less than 3 percent and there was no change for 1995. California fuel, power and water costs have increased every year since 1987, except for 1995, and have exceeded the previous 1985 peak in all of the 1992-1995 period.

\section{Secondary Recovery}

Table 4 provides a summary of.the composite secondary oil recovery operating costs for west Texas. The average aggregated lease costs (10 producing and 11 injection wells) for all depths increased about 6 percent from 1992 to 1995, almost all of which was from the 1993-1994 increase. The largest 1993-1994 changes were in normal daily expense, which ranged from an 8 percent increase for 2,000- and 4,000-foot wells to a 12 percent increase for 8,000 -foot wells. Fuel, power, and water costs for secondary recovery operations increased by almost 18 percent for the 
Table 3. Summary of Direct Annual Operating Costs and Composite Indices for Primary Oil Recovery Operations (10 Producing Wells)

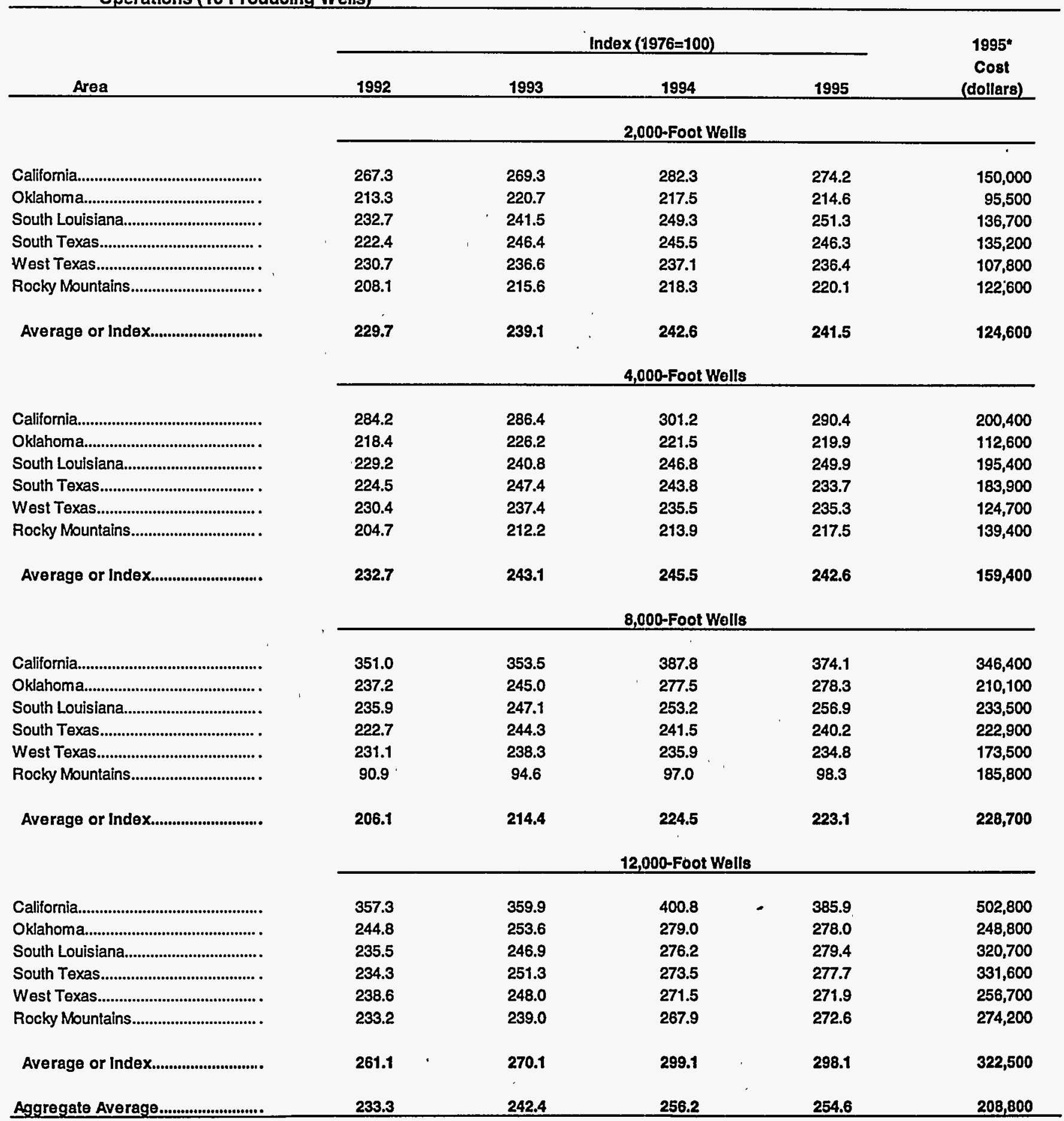

- Preliminary

Note: Reported average or aggregate average indices are indices of the average coste. They are not an average of tho index velues.

Source: Energy Information Administration, Office of Oll and Gas 
Figure 10. Aggregate Operating Costs for Primary Oil Recovery Operations, 1992-1995

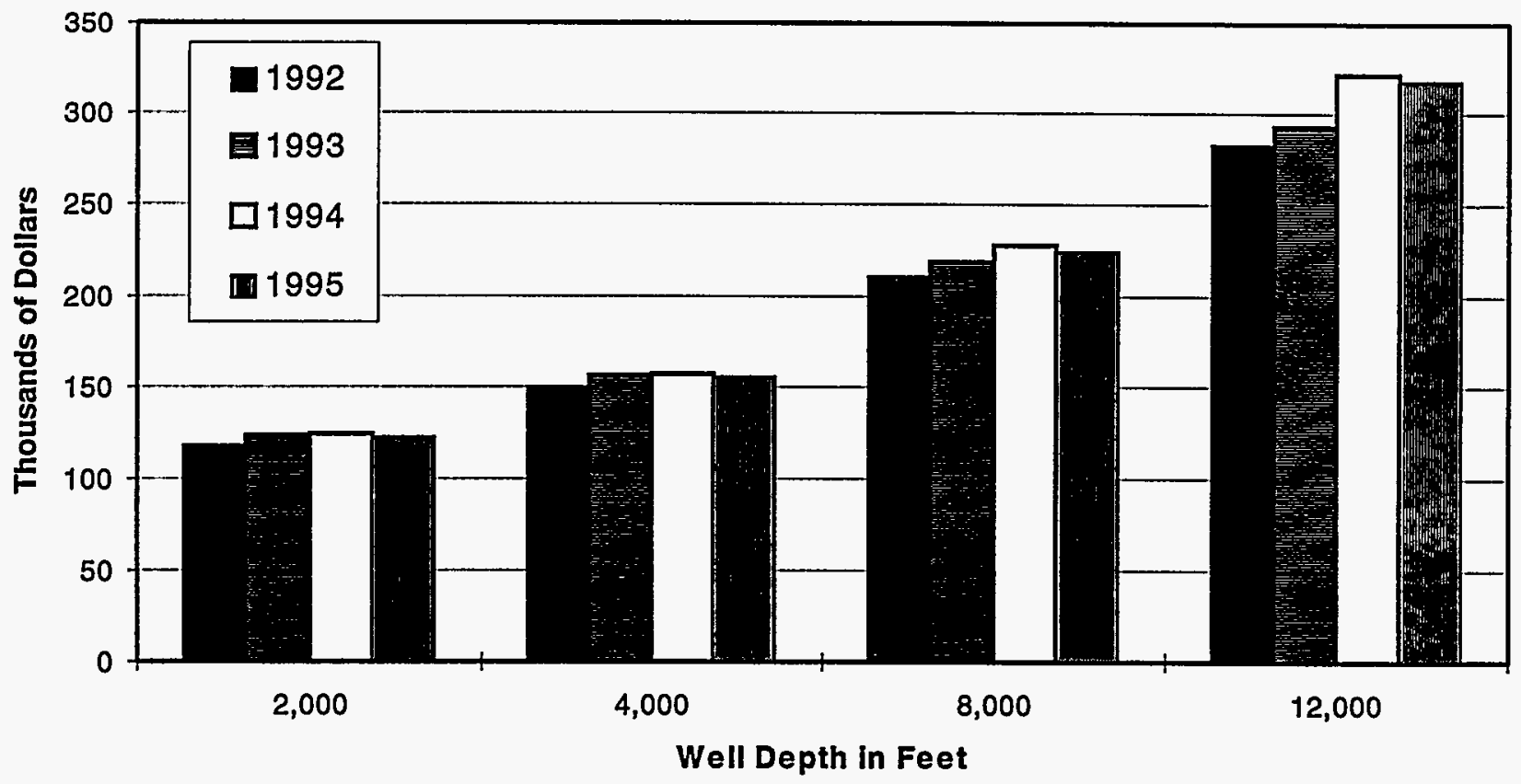

Source: Table 3

Figure 11. Annual Operating Costs for Secondary Oll Recovery In West Texas, 1992-1995 (10 Producing and 11 Injection Wells)

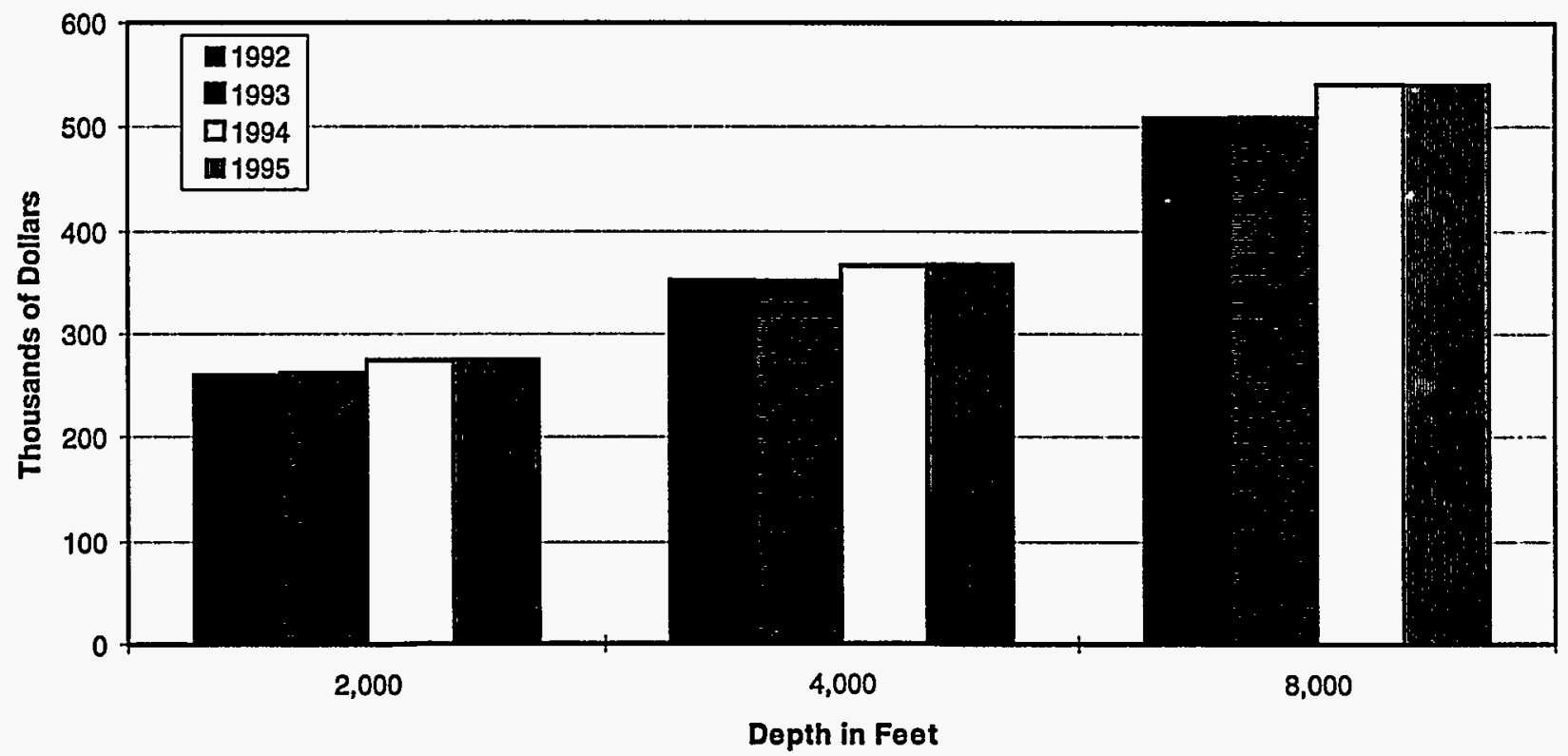

Source: Table 4 
Table 4. Summary of Direct Annual Operating Costs and Composite Indices for Secondary Oil Recovery Operations in West Texas

\begin{tabular}{|c|c|c|c|c|c|}
\hline \multirow[b]{2}{*}{ Component } & \multicolumn{4}{|c|}{ Index $(1976=100)$} & \multirow{2}{*}{$\begin{array}{c}\text { 1995* } \\
\text { Cost } \\
\text { (dollars) }\end{array}$} \\
\hline & 1992 & 1993 & 1994 & 1995 & \\
\hline & \multicolumn{5}{|c|}{ 2,000-Foot Wells } \\
\hline 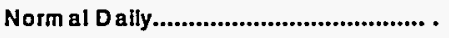 & 254.1 & 255.9 & 277.6 & 278.4 & 171,800 \\
\hline 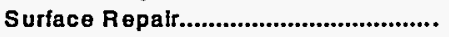 & 234.1 & 229.5 & 231.8 & 232.3 & 50,400 \\
\hline 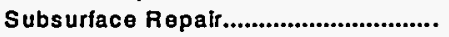 & 197.1 & 198.2 & 195.2 & 196.0 & 53,300 \\
\hline \multirow[t]{2}{*}{ Total or Index.................................. } & 236.2 & 236.5 & 248.4 & 249.1 & 275,500 \\
\hline & \multicolumn{5}{|c|}{ 4,000-Foot Wells } \\
\hline 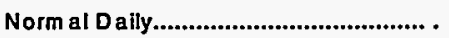 & 314.2 & 315.1 & 340.1 & 341.1 & 208,400 \\
\hline 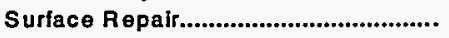 & 238.5 & 233.8 & 236.7 & 237.3 & 81,400 \\
\hline 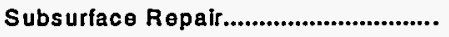 & 193.1 & 193.9 & 191.2 & 192.2 & 78,400 \\
\hline \multirow[t]{2}{*}{ Total or Index................................ } & 258.9 & 258.3 & 269.5 & 270.3 & 368,200 \\
\hline & \multicolumn{5}{|c|}{8,000 -Foot Wells } \\
\hline 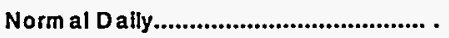 & 303.9 & 305.0 & 341.9 & 342.6 & 301,800 \\
\hline Surface Repair.......................................... & 240.5 & 236.8 & 239.7 & 239.7 & 91,100 \\
\hline 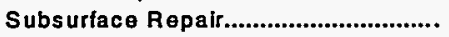 & 199.3 & 199.7 & 196.8 & 197.6 & 149,000 \\
\hline 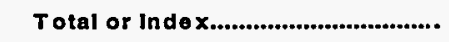 & 252.8 & 252.8 & 268.3 & 268.9 & 541,900 \\
\hline Aggregate Average ............................. . & 250.6 & 250.5 & 263.8 & 264.5 & 395,200 \\
\hline
\end{tabular}

- Prellm inary

Note: Reported average or aggregate average indices are indlces of the average costs. They are net an average of the index values.

Source: Energy Information Administration, Otfice of Oil and $G$ as

Table 5. Summary of Direct Annual Operating Costs and Composite Indices per Platform--Gulf of Mexico (10,500-Foot True Vertical Depth Wells)

\begin{tabular}{|c|c|c|c|c|c|}
\hline \multirow[b]{2}{*}{ Water Depth } & \multicolumn{4}{|c|}{ Index $(1976=100)$} & \multirow{2}{*}{$\begin{array}{c}\text { 1995* } \\
\text { Cost } \\
\text { (dolfars) }\end{array}$} \\
\hline & 1992 & 1993 & 1994 & 1995 & \\
\hline & \multicolumn{5}{|c|}{ 12-Slot Platforms } \\
\hline 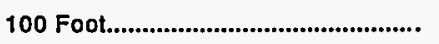 & 211.4 & 219.3 & 227.6 & 233.6 & $3,178,500$ \\
\hline 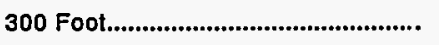 & 209.5 & 217.6 & 226.0 & 231.9 & $3,331,000$ \\
\hline \multirow[t]{2}{*}{ 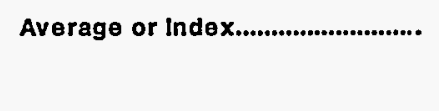 } & 207.2 & 215.1 & 223.3 & 229.2 & $3,254,750$ \\
\hline & \multicolumn{5}{|c|}{ 18-Slot Platforms } \\
\hline 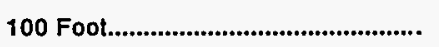 & 204.1 & 212.8 & 221.5 & 228.4 & $3,894,000$ \\
\hline 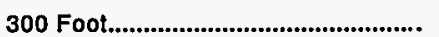 & 197.9 & 206.8 & 215.3 & 222.3 & $3,967,500$ \\
\hline 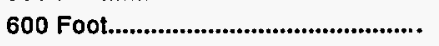 & 193.2 & 202.7 & 212.6 & 218.9 & $4,442,280$ \\
\hline Average or Index......................... & 184.0 & 192.5 & 200.9 & 207.2 & $4,101,260$ \\
\hline Aggregate Average ........................ & 191.6 & 199.8 & 208.2 & 214.3 & $3,762,700$ \\
\hline
\end{tabular}

- Preliminary

Note: Reported average or aggregate average indices are indices of the average costs. They are net an avorage of the ind $\theta x$ values.

Source: Energy Information Administration, Office of Oil and Gas 
Figure 12. Fuel, Power, and Water, Cost Indices for 12,000-Foot Oil Wells in California and Rocky Mountains

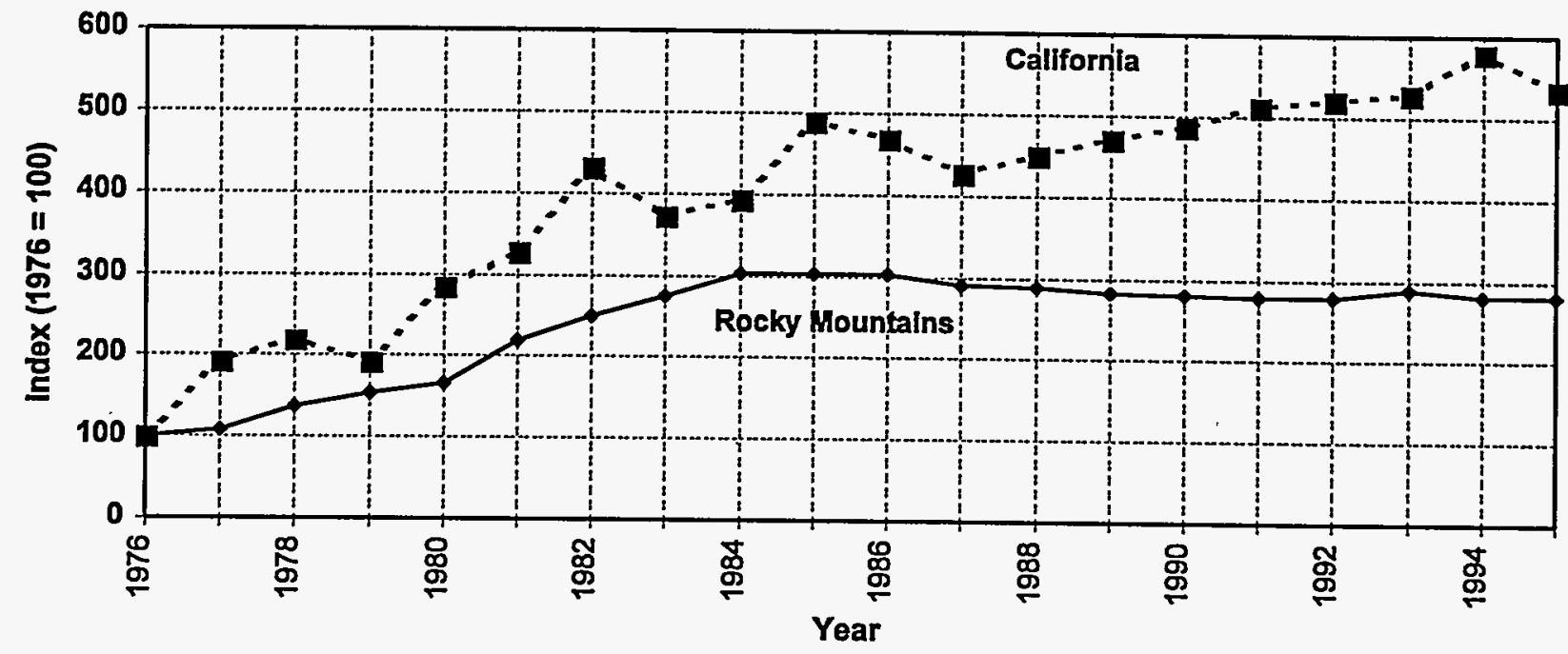

Source: Energy Information Administration, Ofrice of Oll and Gas.

Figure 13. Fuel, Power, and Water Cost Indices for Primary and Secondary Operating Costs for 4,000-foot Wells in West Texas

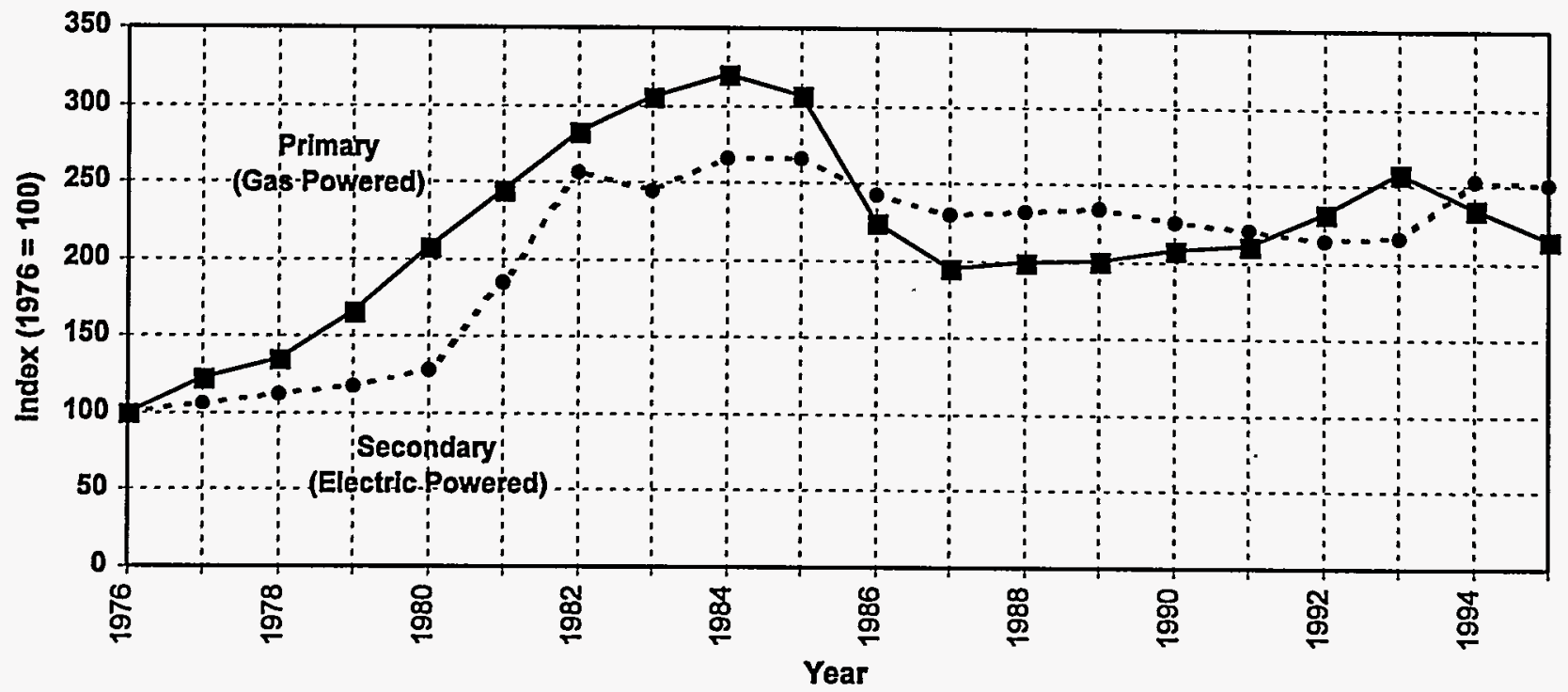

Source: Enargy Information Administration, Ofice of Oll and Gas. 
1992-1994 period and dropped 1 percent in 1995. Fuel, power, and water costs for primary recovery operations in this region increased 7 percent from 1992 to 1994 and fell 9 percent in 1995. The differences in the changes for fuel, power, and water costs occurred because engines power by natural gas engines were the prime movers for primary recovery operations in this region, and electric motors were used for secondary recovery operations. As an example, Figure 13 shows fuel, power, and water cost indices for primary and secondary oil recovery in west Texas for 4,000foot wells. Peaks for both of these indices occurred in the 1984-1985 period.

\section{Offshore Operations}

Table 5 presents a summary of annual operating costs and composite indices for offshore production operations in the Gulf of Mexico for wells with a true vertical depth of 10,500 feet. Production from offshore installations includes large gas volumes compared to the average onshore lease. The aggregate average of costs at all water depths increased by 12 percent from 1992 to $\$ 3,762,700$ per platform in 1995.

\section{Equipment Costs for Gas Leases}

Equipment and operating costs for gas leases producing from depths of 2,000,4,000,8,000,12,000, and 16,000 feet, were determined for 6 onshore regions of the lower 48 States (Figure 2). For each region and depth, costs and indices for equipment for a one-well lease were determined for representative or average gas production rates. Costs and indices were also calculated for a higher and, where possible, for a lower production rate. Composite indices and costs for equipment are presented for a one-well lease with production rates of $50,250,500,1,000,5,000$, and 10,000 Mcf of gas per day by depth and region. Figure 14 displays the average equipment costs by rate of production and well depth for 1995. There is a large difference between the equipment costs for some production rates and depths, such as wells of various depths producing $250 \mathrm{Mcf}$ per day. This difference is the result of variations in the type and size of equipment needed in different regions, for different depths, and for different production rates. For example, dehydrators and line heaters are needed in cold climates but may not be needed in more temperate climates.

The indices for the aggregate costs of gas lease equipment for all depths and regions turned downward for the 19921995 period, with a drop of about 8 percent in 1994. The 3 percent increase in 1995 resulted from an overall aggregate average gas lease equipment increase to $\$ 44,300$ (Table 6).

Tables 7 through 12 present summaries of composite gas lease equipment costs and indices for a given production rate by depth and region. For each production rate, the costs are summed and averaged for the selected regions and depths. These average costs and the corresponding indices are presented in each table. The 1992-1995 change in equipment costs ranged from an increase of 5 percent for wells producing $10 \mathrm{MMcf}$ of gas per day to a decrease of 8 percent for wells flowing $1 \mathrm{MMcf}$ of gas per day.

Table 13 contains gas lease equipment costs aggregated by depth. Changes in gas equipment costs from 1992 to 1995 were positive for 2,000-foot wells, where costs rose about 4 percent and ranged from decreases of 1 to 6 percent for other depths. The dominant factor in determining gas well equipment costs is the production capacity of the equipment. Figure 15 illustrates the aggregate average gas well equipment costs for 1990 through 1993 by production rate. The significant feature of Table 13 is the substantial drop in costs from 1993 to 1994, when year to year decreases were from 4 to 11 percent.

\section{Operating Costs for Gas Leases}

Operating costs for gas leases aggregated for all depths, regions and production rates are shown in Table 14. There was an increase of 4 percent from 1992 to 1995 , to $\$ 23,000$. Tables 15 through 20 are summaries of composite costs and indices for operating a gas lease. Each table is a summary for one production rate for the same depth and region used for lease equipment costs. For each depth and production rate, the individual operating costs by region were averaged and indices were calculated. From 1992 to 1995, wells producing 250 thousand cubic feet per day exhibited an operating cost increase of about 3 percent, while costs for wells producing at a rate of 10 million cubic feet per day rose at about 7 percent. Note that both equipment and operating cost increases were at a maximum for wells in the latter group.

Well depth has more effect on gas well operating costs than on equipment costs, since depth is a major factor in the cost of down-hole repairs, the amount of chemicals used, and other maintenance cost components. However, the changes in operating costs aggregated by depth from 1992 through 1995 show little variation across time. The annual changes ranged from only 4 to 5 percent from 1992 to 1995 . The annual gas well operating costs aggregated by depth are shown in Table 21.

Figure 17 depicts the aggregate average annual gas well operating costs by depth and producing rate for 1995 . Operating costs decreased as the producing rate increased from 250 to 500 thousand cubic feet of gas per day in 8,000 and 12,000-foot wells. This is a result of the well design and the completion techniques used. Wells producing at 500 thousand cubic feet of gas per day, or more, were considered to be completed with packers. Packers protect the casingtubing annulus and the casing wellhead from the bottomhole pressure and any corrosive properties of the well's 
fluids. With these flow rates, the tubing flow velocity is sufficient to remove the well liquids which accumulate in the tubing. Either tubing displacement or corrosion inhibitor squeeze jobs can be used to protect the production string from corrosion or scale deposition. Wells producing at rates of 250 thousand cubic feet of gas per day or less have lower tubing flow velocities which are not always adequate to remove accumulated liquids from the well. Increasing liquid levels usually cause reduced gas production. Therefore, these wells were considered to be completed without packers to permit fluids to be forced up the tubing by expansion of the compressed gas in the casing-tubing annulus. Because the gas wells which produce at lower flow rates have no packers, the casing-tubing annulus is exposed to the corrosive properties of the well fluids and often needs chemical protection. Tubing displacement and corrosion inhibitor squeeze jobs are not effective without a packer, or making them effective would be cost prohibitive. Therefore, continuous chemical injection down the casing-tubing annulus is a common practice. This involves surface chemical injection pumps, maintenance, and larger volumes of chemicals. Therefore, wells which produce less than 250 thousand cubic feet of gas per day have higher fuel, chemical and disposal costs, and higher surface maintenance costs. 
Figure 14. Annual Gas Well Equipment Costs by Well Depth and Production Rate (1995)

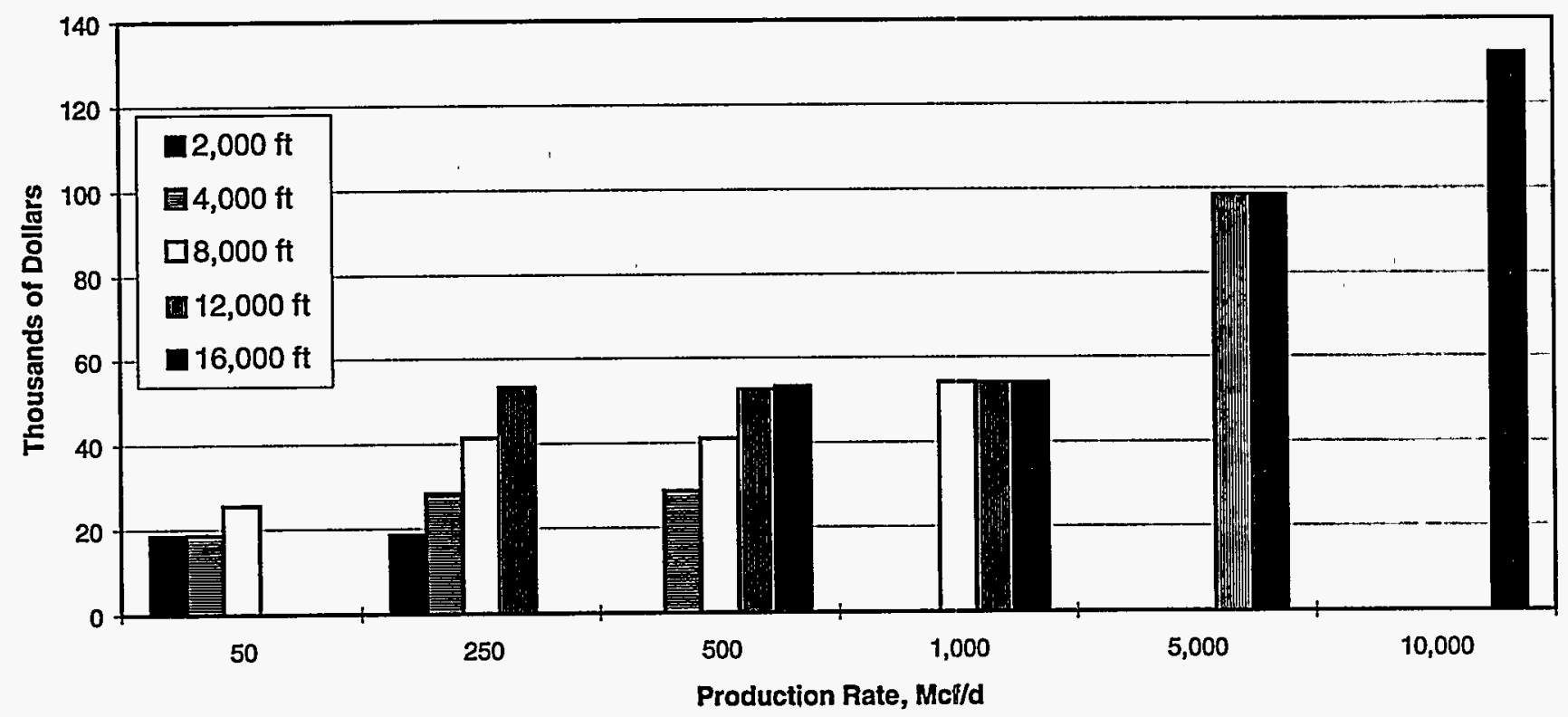

Source: Tables 7 through 12.

Figure 15. Aggregate Average Equipment Costs for a One-Well Gas Lease by Production Rate, 1992-1995

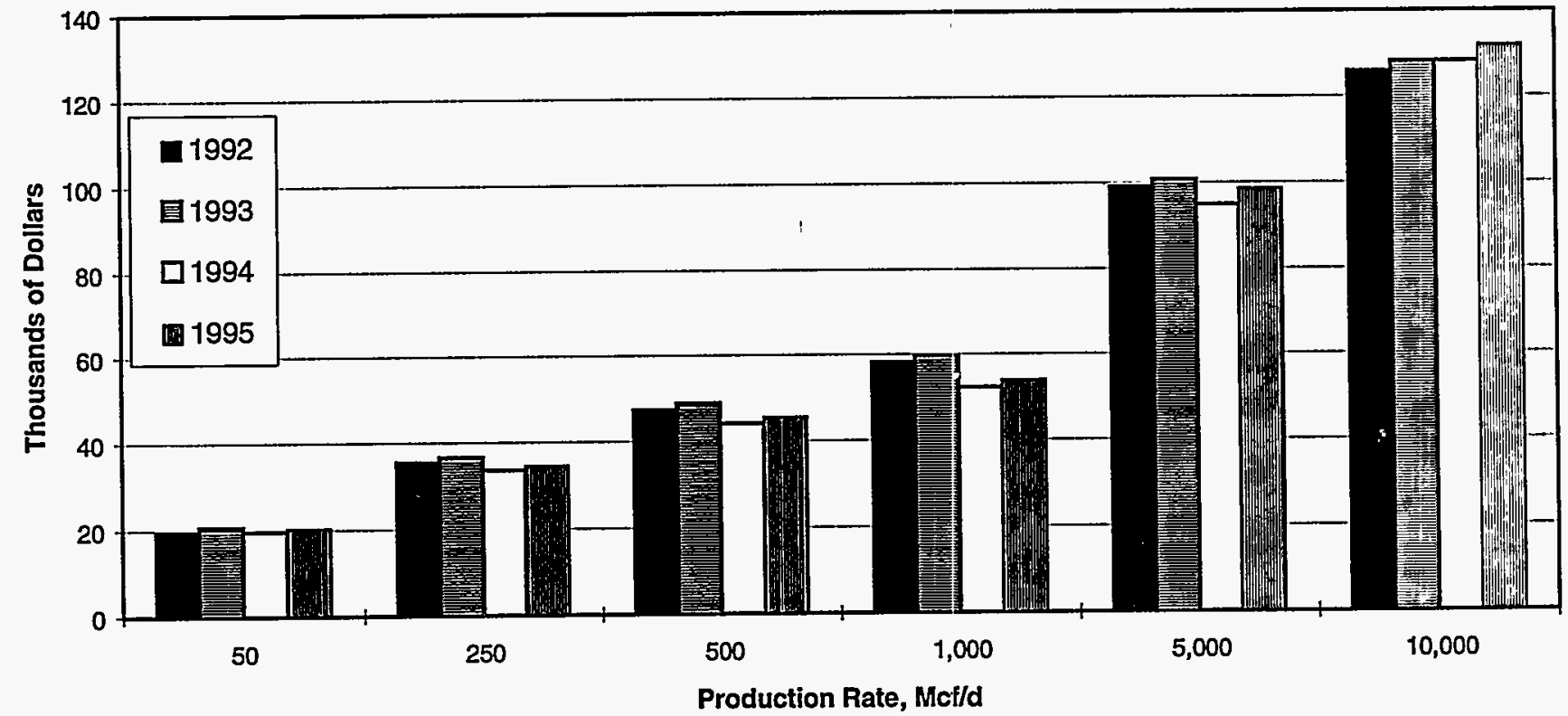

Source: Tables 7 through 12. 
Table 6. Average Equipment Costs and Indices for Gas Leases Aggregated for All Depths, Areas and Production Rates (One Producing Well)

\begin{tabular}{|c|c|c|c|c|}
\hline \multicolumn{4}{|c|}{ Index $(1976=100)$} & \multirow{2}{*}{$\begin{array}{c}\text { 1995* } \\
\text { Cost } \\
\text { (dollars) }\end{array}$} \\
\hline 1992 & 1993 & 1994 & 1995 & \\
\hline 194.9 & 200.4 & 183.3 & 189.3 & 44,300 \\
\hline
\end{tabular}

Aggregate average for

all Production Rates...................................

- Preliminary

Note: Reported average or aggregate average indices are indices of the average costs. They are net an average of the index values.

Source: Energy Information Administration, Ofice of Oil and Gas

Table 7. Summary of Gas Lease Equipment Costs and Composite Indices for One Well Producing 50 Thousand Cubic Feet per Day

\begin{tabular}{|c|c|c|c|c|c|}
\hline \multirow[b]{2}{*}{ Area } & \multicolumn{4}{|c|}{ Index $(1976=100)$} & \multirow{2}{*}{$\begin{array}{c}\text { 1995* } \\
\text { Cost } \\
\text { (dollars) }\end{array}$} \\
\hline & 1992 & 1993 & 1994 & 1995 & \\
\hline & \multicolumn{5}{|c|}{ 2,000-Foot Wells } \\
\hline Mid-Continent............................................... & 176.2 & 184.2 & 177.2 & 183.2 & 18,500 \\
\hline North Louisiana.......................................... & 167.6 & 174.3 & 168.6 & 176.2 & 18,500 \\
\hline South Loulsiana........................................... & 167.6 & 174.3 & 168.6 & 176.2 & 18,500 \\
\hline Rocky Mountains.......................................... . & 163.4 & 171.4 & 164.3 & 170.5 & 19,100 \\
\hline 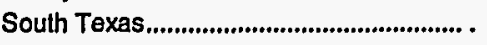 & 168.9 & 179.6 & 171.8 & 177.7 & 18,300 \\
\hline West Texas..................................................... & 174.3 & 186.1 & 176.2 & 182.2 & 18,400 \\
\hline \multirow[t]{2}{*}{ Average or Index................................. } & 168.6 & 177.1 & 170.5 & 177.1 & 18,600 \\
\hline & \multicolumn{5}{|c|}{ 4,000-Foot Wells } \\
\hline Mid-Continent............................................... & 176.2 & 184.2 & 177.2 & 183.2 & 18,500 \\
\hline 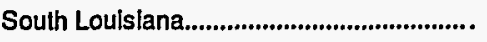 & 167.6 & 174.3 & 168.6 & 176.2 & 18,500 \\
\hline Rocky Mountains......................................... & 163.4 & 171.4 & 164.3 & 170.5 & 19,100 \\
\hline South Texas................................................... . & 168.9 & 179.6 & 171.8 & 177.7 & 18,300 \\
\hline West Texas..................................................... . & 174.3 & 186.1 & 176.2 & 182.2 & 18,400 \\
\hline \multirow[t]{2}{*}{ Average or Index................................... } & 170.2 & 179.8 & 172.1 & 178.8 & 18,600 \\
\hline & \multicolumn{5}{|c|}{ 8,000-Foot Wells } \\
\hline 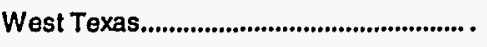 & 193.9 & 203.1 & 186.3 & 194.7 & 25,500 \\
\hline \multicolumn{6}{|l|}{ Aggregate } \\
\hline Average for Production Rate............... . & 172.0 & 180.4 & 172.0 & 178.5 & 19,100 \\
\hline
\end{tabular}

- Preliminary

Note: Reported average or aggregate average indices are indices of the average costs. They are net an average of the index values.

Source: Energy Iniormation Administration, Office of Oil and Gas 
Table 8. Summary of Gas Lease Equipment Costs and Composite Indices for One Well Producing 250 Thousand Cubic Feet per Day

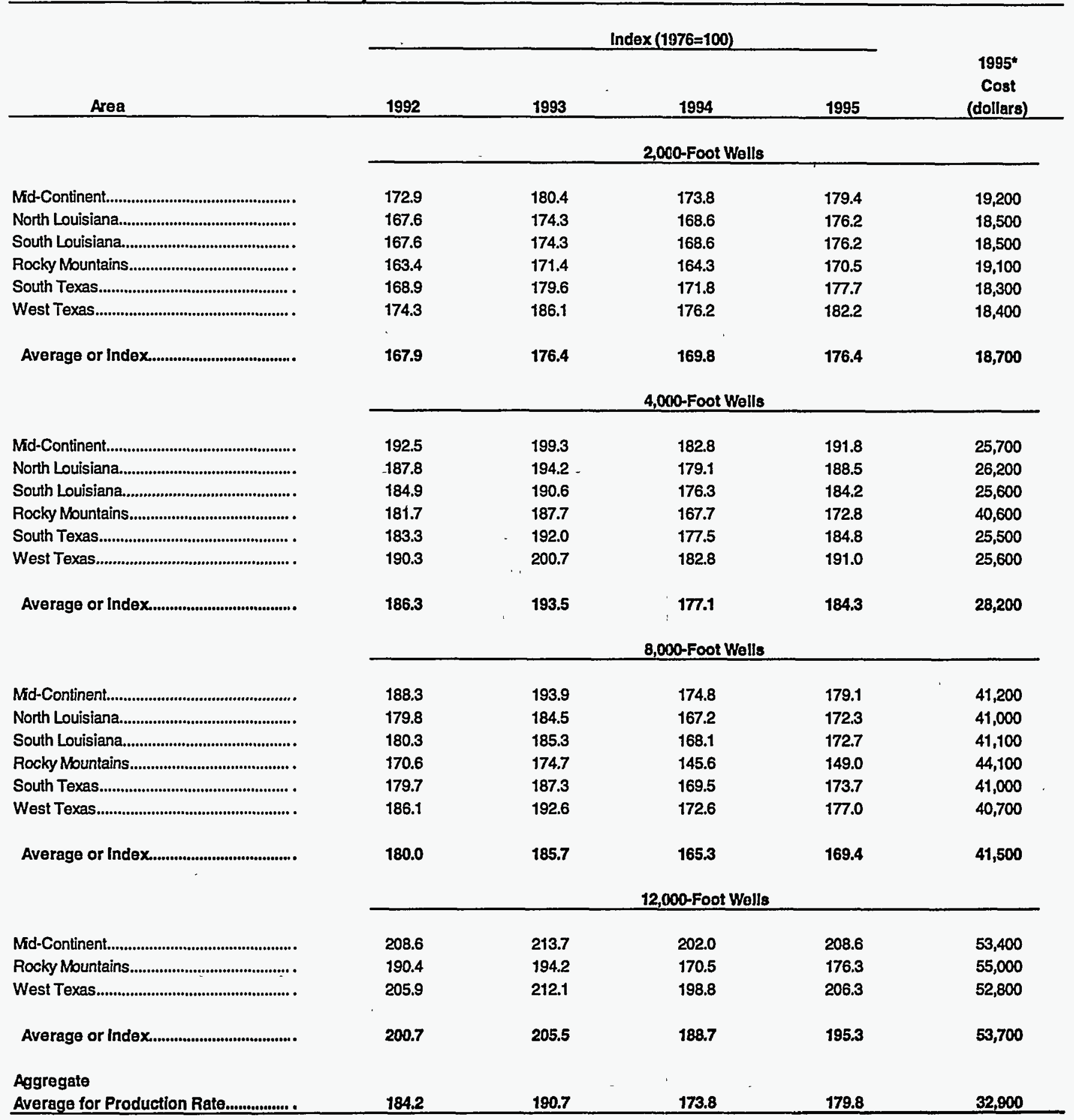

- Preliminary

Note: Reported average or aggregate average indices are indices of the average costs. They are not an average of the index values.

Source: Energy Information Administration, Office of Oil and Gas 


\begin{tabular}{|c|c|c|c|c|c|}
\hline \multirow[b]{2}{*}{ Area } & \multicolumn{4}{|c|}{ Index $(1976=100)$} & \multirow{2}{*}{$\begin{array}{c}1995^{\star} \\
\text { Cost } \\
\text { (dollars) }\end{array}$} \\
\hline & 1992 & 1993 & 1994 & 1995 & \\
\hline & \multicolumn{5}{|c|}{ 4,000-Foot Wells } \\
\hline 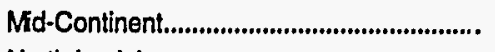 & 198.4 & 204.7 & 187.4 & 196.9 & 25,000 \\
\hline 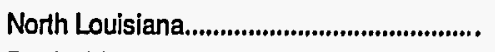 & 169.2 & 176.9 & 170.2 & 176.9 & 18,400 \\
\hline 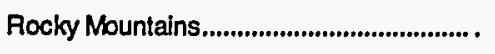 & 173.4 & 178.0 & 146.9 & 150.3 & 43,000 \\
\hline \multirow[t]{2}{*}{ Average or Index } & 179.1 & 184.9 & 161.6 & 167.4 & 28,800 \\
\hline & \multicolumn{5}{|c|}{$8,000-$ Foot Wells } \\
\hline 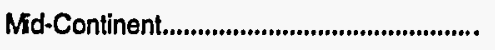 & 192.5 & 197.4 & 178.9 & 182.9 & 41,700 \\
\hline 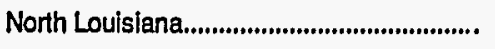 & 180.0 & 185.1 & 167.2 & 171.9 & 40,400 \\
\hline South Louisiana.................................................. & 180.0 & 185.1 & 167.2 & 171.9 & 40,400 \\
\hline 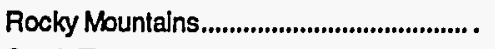 & 172.9 & 177.4 & 147.2 & 150.7 & 43,400 \\
\hline South Texas..................................................... . & 179.8 & 187.1 & 168.7 & 173.0 & 40,300 \\
\hline West Texas...................................................... & 185.5 & 191.7 & 171.5 & 175.9 & 40,100 \\
\hline \multirow[t]{2}{*}{ Average or Index } & 181.7 & 187.1 & 166.0 & 170.5 & 41,100 \\
\hline & \multicolumn{5}{|c|}{ 12,000-Foot Wells } \\
\hline 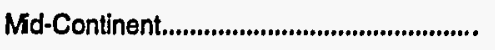 & 211.6 & 216.9 & 204.8 & 211.6 & 52,700 \\
\hline 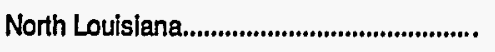 & 203.9 & 209.0 & 197.7 & 205.1 & 52,500 \\
\hline 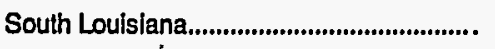 & 203.9 & 209.0 & 197.7 & 205.1 & 52,500 \\
\hline 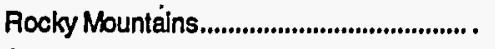 & 193.1 & 197.4 & 172.7 & 178.6 & 54,300 \\
\hline 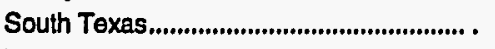 & 203.9 & 211.0 & 199.2 & 206.3 & 52,400 \\
\hline 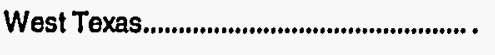 & 209.6 & 215.7 & 202.4 & 209.6 & 52,200 \\
\hline \multirow[t]{2}{*}{ Average or Index................................... } & 204.2 & 209.6 & 195.4 & 202.3 & 52,800 \\
\hline & \multicolumn{5}{|c|}{16,000 -Foot Wells } \\
\hline Mid-Continent......................................................... & 197.7 & 202.0 & 177.2 & 182.6 & 54,400 \\
\hline 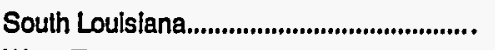 & 203.9 & 209.0 & 197.7 & 205.1 & 52,500 \\
\hline 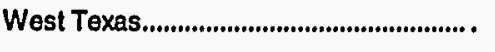 & 195.0 & 199.7 & 174.5 & 180.2 & 53,700 \\
\hline Average or Index......................................... & 198.6 & 203.2 & 182.4 & 188.4 & 53,500 \\
\hline \multicolumn{6}{|l|}{ Aggregate } \\
\hline Average for Production Rate.................. . & 192.2 & 197.5 & 178.7 & 184.4 & 45,000 \\
\hline
\end{tabular}

- Preliminary

Note: Reported average or aggregate average indices are indices of the average costs. They are not an average of the index values.

Source: Energy information Administration, Office of Oil and Gas 
Table 10. Summary of Gas Lease Equipment Costs and Composite Indices for One Well Producing 1 Million Guble Feot per Day

\begin{tabular}{|c|c|c|c|c|c|}
\hline \multirow[b]{2}{*}{ Area } & \multicolumn{4}{|c|}{ Index $(1976=100)$} & \multirow{2}{*}{$\begin{array}{c}1995^{\circ} \\
\text { Cost } \\
\text { (dollars) }\end{array}$} \\
\hline & 1992 & 1993 & 1994 & 1995 & \\
\hline & \multicolumn{5}{|c|}{$8,000 . F o o t$ Wells } \\
\hline $\begin{array}{l}\text { South Louisiana. } \\
\text { South Texas }\end{array}$ & $\begin{array}{l}192.4 \\
193.4\end{array}$ & $\begin{array}{l}196.4 \\
199.0\end{array}$ & $\begin{array}{l}172.7 \\
174.1\end{array}$ & $\begin{array}{l}178.3 \\
179.7\end{array}$ & $\begin{array}{l}54,200 \\
54,100\end{array}$ \\
\hline \multirow[t]{2}{*}{ 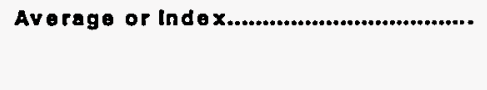 } & 192.7 & 197.4 & 173.3 & 178.9 & 54,200 \\
\hline & \multicolumn{5}{|c|}{ 12,000-Foot Wells } \\
\hline 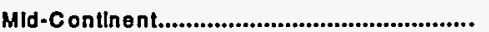 & 197.7 & 202.0 & 177.2 & 182.6 & 54,400 \\
\hline 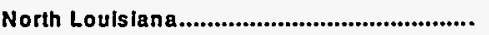 & 192.4 & 196.4 & 172.7 & 178.3 & 54,200 \\
\hline 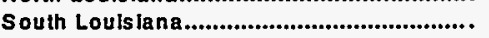 & 192.4 & 196.4 & 172.7 & 178.3 & 54,200 \\
\hline R ocky Mountains ............................................ & 193.1 & 197.4 & 172.7 & 178.6 & 54,300 \\
\hline 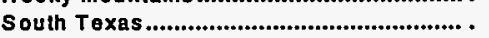 & 192.7 & 198.3 & 173.5 & 179.1 & 54,100 \\
\hline 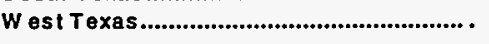 & 195.0 & 199.7 & 174.5 & 180.2 & 53,700 \\
\hline \multirow[t]{2}{*}{ 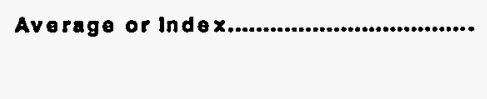 } & 193.7 & 198.0 & 173.8 & 179.5 & 54,200 \\
\hline & \multicolumn{5}{|c|}{$16,000-F o$ ot V/olls } \\
\hline 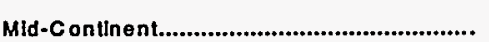 & 197.7 & 202.0 & 177.2 & 182.6 & 54,400 \\
\hline 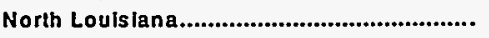 & 192.4 & 196.4 & 172.7 & 178.3 & 54,200 \\
\hline 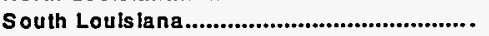 & 192.4 & 196.4 & 172.7 & 178.3 & 54,200 \\
\hline 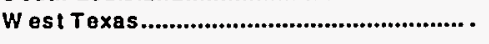 & 195.0 & 199.7 & 174.5 & 180.2 & 53,700 \\
\hline Average or Index & 194.4 & 198.7 & 174.4 & 179.7 & 54,100 \\
\hline $\begin{array}{l}\text { Aggregate } \\
\text { Average tor Production Rate................ }\end{array}$ & 193.7 & 198.0 & 173.8 & 179.1 & 54,100 \\
\hline
\end{tabular}

- Prolim inary

Note: Reported average or aggregate average indices are indices of the average costs. They are net an average of the index values.

Source: Energy Inform ation Adm inistration. Office of Oll and $G$ as

Table 11. Summary of Gas Lease Equipment Costs and Composite Indices for One Well Producing 5 Million Cubic Feet per Day

\begin{tabular}{|c|c|c|c|c|c|}
\hline \multirow[b]{2}{*}{ Area } & \multicolumn{4}{|c|}{ Index $(1976=100)$} & \multirow{2}{*}{$\begin{array}{c}1995^{*} \\
\text { Cost } \\
\text { (dollars) }\end{array}$} \\
\hline & 1992 & 1993 & 1994 & 1995 & \\
\hline & \multicolumn{5}{|c|}{ 12,000-Foot Wells } \\
\hline 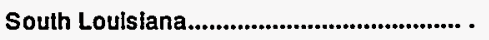 & 222.6 & 225.8 & 212.8 & 220.9 & 98,500 \\
\hline 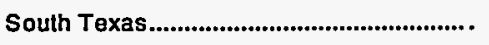 & 222.7 & 227.3 & 213.5 & 221.6 & 98,400 \\
\hline \multirow[t]{2}{*}{ 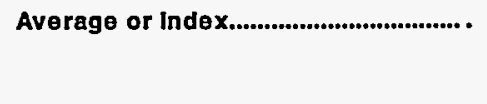 } & 222.7 & 226.5 & 213.3 & 221.3 & 98,500 \\
\hline & \multicolumn{5}{|c|}{ 16,000-Foot Wells } \\
\hline 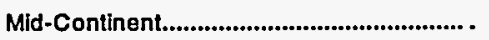 & 226.9 & 230.1 & 216.9 & 224.8 & 98,700 \\
\hline North Louisiana.............................................. . & 222.6 & 225.8 & 212.8 & 220.9 & 98,500 \\
\hline 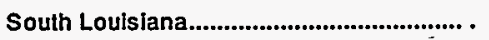 & 222.6 & 225.8 & 212.8 & 220.9 & 98,500 \\
\hline 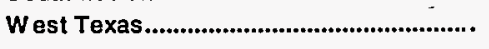 & 224.6 & 228.7 & 214.8 & 222.8 & 97,800 \\
\hline 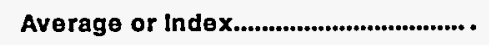 & 223.9 & 227.3 & 214.0 & 222.1 & 98,400 \\
\hline \multicolumn{6}{|l|}{ Aggregate } \\
\hline Average for Production Rate................ & 223.9 & 227.3 & 214.0 & 222.1 & 98,400 \\
\hline
\end{tabular}

- Preliminary

Note: Reported average or aggregate average indices are Indices of the average costs. They are not an average of the index values.

Source: Energy Information Administration, Office of Oll and Gas 
Table 12. Summary of Gas Lease Equipment Costs and Composite Indices for One Well Producing 10 Million Cubic Feet per Day

\begin{tabular}{|c|c|c|c|c|c|}
\hline \multirow[b]{2}{*}{ Area } & \multicolumn{4}{|c|}{ Index $(1976=100)$} & \multirow{2}{*}{$\begin{array}{c}\text { 1995* } \\
\text { Cost } \\
\text { (dollars) }\end{array}$} \\
\hline & 1992 & 1993 & 1994 & 1995 & \\
\hline North Louisiana..................................... & 212.8 & 216.2 & 216.2 & 222.4 & 131,900 \\
\hline
\end{tabular}

- Prelliminary

Note; Reported average or aggregate average Indices are Indices of the average costs. They are not an average of the index values.

Source: Energy Information Administration, Office of Oll and Gas

Table 13. Summary of Aggregate Average Gas Lease Equipment Costs by Well Depth (1992-1995)

\begin{tabular}{|c|c|c|c|c|}
\hline \multirow{2}{*}{$\begin{array}{c}\text { Well Depth } \\
\text { (feet) }\end{array}$} & \multicolumn{4}{|c|}{ Average Costs (dollars) } \\
\hline & 1992 & 1993 & 1994 & 1995 \\
\hline $2,000 \ldots \ldots \ldots \ldots \ldots \ldots \ldots \ldots \ldots \ldots \ldots \ldots \ldots \ldots \ldots \ldots \ldots \ldots \ldots \ldots \ldots \ldots$ & 17,800 & 18,700 & 17,900 & 18,600 \\
\hline 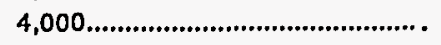 & 25,200 & 26,200 & 24,000 & 24,900 \\
\hline 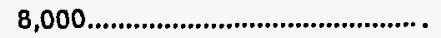 & 44,600 & 46,000 & 40,800 & 41,900 \\
\hline 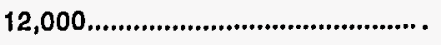 & 60,800 & 62,300 & 56,800 & 58,800 \\
\hline 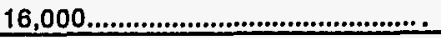 & 77,200 & 78,600 & 72,700 & 75,200 \\
\hline
\end{tabular}

- Prellminary

Source: Energy Information Adm inistration, Office of Ofl and Gas 
Table 14. Average Operating Costs and Indlces for Gais Leases Aggregated for All Depths, Areas and Production Rates (One Producing Well)

\begin{tabular}{|c|c|c|c|c|c|}
\hline \multirow[b]{2}{*}{ Area } & \multicolumn{4}{|c|}{ Index $(1976=100)$} & \multirow{2}{*}{$\begin{array}{c}\text { 1995" } \\
\text { Cost } \\
\text { (dollars) }\end{array}$} \\
\hline & 1992 & 1993 & 1994 & 1995 & \\
\hline $\begin{array}{l}\text { Aggregate Averege for } \\
\text { all Production Rates................. }\end{array}$ & 208.5 & 215.1 & 215.1 & 217.0 & 23,000 \\
\hline
\end{tabular}

- Preliminary

Note: Reported average or aggregate average indices are Indices of the average costs. They are not an average of the index values.

Source: Energy Information Adm inistration, Office of Oll and Gas

Table 15. Summary of Gas Lease Operating Costs and Composite Indices for One Well Producing 50 Thousand Cubic Feet per Day

\begin{tabular}{|c|c|c|c|c|c|}
\hline \multirow[b]{2}{*}{ Area } & \multicolumn{4}{|c|}{ Index $(1976=100)$} & \multirow{2}{*}{$\begin{array}{c}\text { 1995* } \\
\text { Cost } \\
\text { (dollar8) }\end{array}$} \\
\hline & 1992 & 1993 & 1994 & 1995 & \\
\hline & \multicolumn{5}{|c|}{ 2,000-Foot Wells } \\
\hline Mid-Continent.................................................... & 258.8 & 270.6 & 270.6 & 273.5 & 9,300 \\
\hline 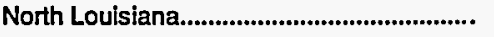 & 207.3 & 214.6 & 219.5 & 222.0 & 9,100 \\
\hline South Louisiana.............................................. & 209.8 & 217.1 & 222.0 & 224.4 & 9,200 \\
\hline Rocky Mountains......................................... & 212.5 & 216.7 & 216.7 & 216.7 & 10,400 \\
\hline South Texas................................................. . & 228.2 & 246.2 & 248.7 & 251.3 & 9,800 \\
\hline 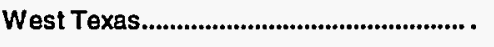 & 247.1 & 244.1 & 247.1 & 247.1 & 8,400 \\
\hline \multirow[t]{2}{*}{ Average or Index................................ } & 222.5 & 230.0 & 232.5 & 235.0 & 9,400 \\
\hline & \multicolumn{5}{|c|}{$4,000-$ Foot Welle } \\
\hline Mid-Continent.................................................. & 240.5 & 250.0 & 250.0 & 252.4 & 10,600 \\
\hline 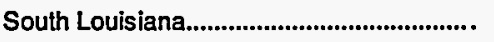 & 210.6 & 217.0 & 221.3 & 227.7 & 10,700 \\
\hline 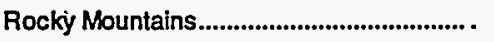 & 208.9 & 212.5 & 214.3 & 216.1 & 12,100 \\
\hline 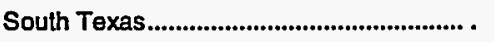 & 226.7 & 246.7 & 246.7 & 248.9 & 11,200 \\
\hline 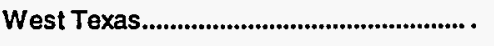 & 246.3 & 246.3 & 246.3 & 246.3 & 10,100 \\
\hline \multirow[t]{2}{*}{ Average or Index................................... } & 226.1 & 234.8 & 234.8 & 237.0 & 10,900 \\
\hline & \multicolumn{5}{|c|}{ 8,000-Foot Wells } \\
\hline 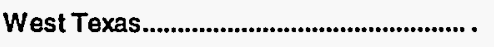 & 227.6 & 227.6 & 225.9 & 227.6 & 13,200 \\
\hline \multicolumn{6}{|l|}{ Aggregate } \\
\hline Average for Productlon Rate................ . & 225.0 & 231.8 & 234.1 & 234.1 & 10,300 \\
\hline
\end{tabular}

- Preliminary

Note: Reported average or aggregate average indices are Indices of the average costs. They are net an average of the index values.

Source: Energy Intormation Administration, Office of Oil and Gas 
Table 16. Summary of Gas Lease Operating Costs and Composite. Indices for One Well Producing 250 Thousand Cublc Feet per Day

\begin{tabular}{|c|c|c|c|c|c|}
\hline \multirow[b]{2}{*}{ Area } & \multicolumn{4}{|c|}{ Index $(1976=100)$} & \multirow{2}{*}{$\begin{array}{c}\text { 1995* } \\
\text { Cost } \\
\text { (dollars) }\end{array}$} \\
\hline & 1992 & 1993 & 1994 & 1995 & \\
\hline & \multicolumn{4}{|c|}{ 2,000-Foot Wells } & \\
\hline Md-Continent.............................................. & 248.9 & 259.6 & 259.6 & 261.7 & 12,300 \\
\hline North Louisiana............................................... & 206.1 & 212.2 & 216.3 & 218.4 & 10,700 \\
\hline South Louisiana.......................................... & 208.2 & 214.3 & 218.4 & 220.4 & 10,800 \\
\hline Rocky Mountains.......................................... & 210.7 & 214.3 & 214.3 & 214.3 & 12,000 \\
\hline 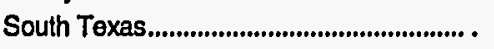 & 223.4 & 238.3 & 240.4 & 242.6 & 11,400 \\
\hline 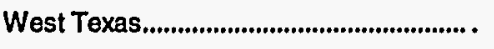 & 238.1 & 235.7 & 238.1 & 238.1 & 10,000 \\
\hline \multirow[t]{2}{*}{ 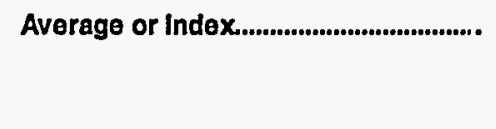 } & 222.9 & 229.2 & 231.3 & 233.3 & 11,200 \\
\hline & \multicolumn{5}{|c|}{ 4,000-Foot Wells } \\
\hline Md-Continent................................................ & 239.4 & 247.0 & 245.5 & 247.0 & 16,300 \\
\hline North Louisiana............................................... & 209.0 & 213.4 & 214.9 & 217.9 & 14,600 \\
\hline South Louisiana............................................... & 213.4 & 217.9 & 219.4 & 223.9 & 15,000 \\
\hline Rocky Mountains......................................... & 210.8 & 216.1 & 215.1 & 217.2 & 20,200 \\
\hline South Texas.................................................. . & 216.9 & 232.3 & 230.8 & 232.3 & 15,100 \\
\hline West Texas.................................................... & 229.5 & 229.5 & 227.9 & 227.9 & 13,900 \\
\hline \multirow[t]{2}{*}{ Average or Index................................. } & 218.6 & 224.3 & 224.3 & 227.1 & 15,900 \\
\hline & \multicolumn{5}{|c|}{8,000 -Foot Wells } \\
\hline Md-Continent............................................. & 236.8 & 243.4 & 242.5 & 241.5 & 25,600 \\
\hline North Louisiana................................................ & 205.9 & 211.0 & 211.9 & 215.3 & 25,400 \\
\hline South Louisiana............................................. & 208.5 & 212.7 & 214.4 & 216.9 & 25,600 \\
\hline Rocky Mountains ........................................ & 211.2 & 217.6 & 215.2 & 215.2 & 26,900 \\
\hline South Texas................................................ & 211.3 & 224.3 & 224.3 & 226.1 & 26,000 \\
\hline West Texas................................................... & 228.3 & 227.4 & 225.5 & 226.4 & 24,000 \\
\hline \multirow[t]{2}{*}{ Average or Index................................. } & 215.7 & 221.7 & 220.9 & 222.6 & 25,600 \\
\hline & \multicolumn{5}{|c|}{ 12,000-Foot Wells } \\
\hline Md-Continent................................................. & 230.1 & 236.8 & 235.3 & 236.8 & 31,500 \\
\hline Rocky Mountains ........................................... & 211.0 & 215.6 & 214.9 & 216.2 & 33,300 \\
\hline West Texas..................................................... & 223.0 & 222.2 & 220.7 & 221.5 & 29,900 \\
\hline Average or Index................................ & 220.6 & 224.1 & 222.7 & 224.1 & 31,600 \\
\hline \multicolumn{6}{|l|}{ Aggregate } \\
\hline Average for Production Rate................ . & 218.4 & 223.0 & 223.0 & 224.1 & 19,500 \\
\hline
\end{tabular}

- Preliminary

Note: Reported average or aggregate average indices are indices of the average costs. They are n.ot an average of the index velues.

Source: Energy information Administration, Otice of Oil and Gas 
Table 17. Summary of Gas Lease Operating Costs and Composite Indices for One Well Producing 500 Thousand Cubic Feet per Day

\begin{tabular}{|c|c|c|c|c|c|}
\hline \multirow[b]{2}{*}{ Area } & \multicolumn{4}{|c|}{ Index $(1976=100)$} & \multirow{2}{*}{$\begin{array}{c}\text { 1995* } \\
\text { Cost } \\
\text { (dollars) }\end{array}$} \\
\hline & 1992 & 1993 & 1994 & 1995 & \\
\hline & \multicolumn{5}{|c|}{ 4,000-Foot Wells } \\
\hline 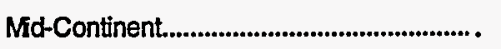 & 251.7 & 263.3 & 261.7 & 263.3 & 15,800 \\
\hline North Louisiana............................................. & 202.8 & 209.9 & 212.7 & 215.5 & 15,300 \\
\hline Rocky Mountains.......................................... . & 203.2 & 211.6 & 211.6 & 212.6 & 20,200 \\
\hline \multirow[t]{2}{*}{ Average or Index................................... . } & 217.3 & 225.3 & 226.7 & 228.0 & 17,100 \\
\hline & \multicolumn{5}{|c|}{ 8,000-Foot Wells } \\
\hline 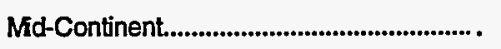 & 248.2 & 259.0 & 256.6 & 256.6 & 21,300 \\
\hline North Louisiana........................................... & 198.1 & 205.8 & 208.7 & 211.5 & 22,000 \\
\hline South Louisiana.......................................... . & 201.9 & 208.7 & 211.5 & 215.4 & 22,400 \\
\hline Rocky Mountains .......................................... & 209.5 & 218.1 & 215.2 & 216.2 & 22,700 \\
\hline 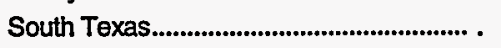 & 176.4 & 193.6 & 192.7 & 194.5 & 21,400 \\
\hline 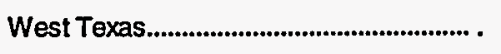 & 231.3 & 230.1 & 227.7 & 227.7 & 18,900 \\
\hline \multirow[t]{2}{*}{ Average or Index..................................... } & 209.2 & 217.3 & 217.3 & 219.4 & 21,500 \\
\hline & \multicolumn{5}{|c|}{ 12,000-Foot Wells } \\
\hline Md-Continent................................................. & 236.9 & 248.5 & 246.6 & 248.5 & 25,600 \\
\hline North Louisiana............................................. . & 194.1 & 200.0 & 203.4 & 208.5 & 24,600 \\
\hline South Louisiana.......................................... & 204.2 & 209.3 & 213.6 & 217.8 & 25,700 \\
\hline 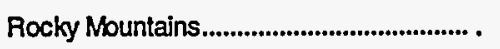 & 208.7 & 215.7 & 215.7 & 217.3 & 27,600 \\
\hline 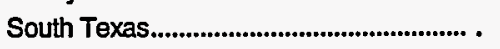 & 209.6 & 227.0 & 228.7 & 231.3 & 26,600 \\
\hline 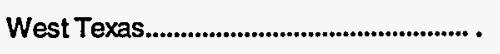 & 223.8 & 222.9 & 220.0 & 221.0 & 23,200 \\
\hline \multirow[t]{2}{*}{ Average or Index................................ } & 213.2 & 220.2 & 221.1 & 224.6 & 25,600 \\
\hline & \multicolumn{5}{|c|}{ 16,000-Foot Wells } \\
\hline 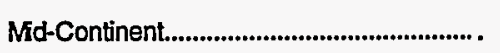 & 229.7 & 239.8 & 236.4 & 236.4 & 27,900 \\
\hline 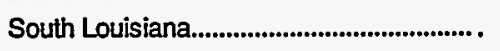 & 199.2 & 204.5 & 208.3 & 212.9 & 28,100 \\
\hline 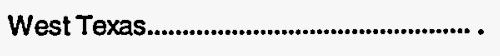 & 221.7 & 221.7 & 219.2 & 220.0 & 26,400 \\
\hline Average or Index.................................. & 217.1 & 222.0 & 221.1 & 223.6 & 27,500 \\
\hline \multicolumn{6}{|l|}{ Aggregate } \\
\hline Average for Production Rate................ . & 223.2 & 231.3 & 231.3 & 233.3 & 23,100 \\
\hline
\end{tabular}

- Preliminary

Note: Reported average or aggregate average indices are indices of the average costs. They are not an average of the index values.

Source: Energy Information Administralion, Office of Oil and Gas 
Table 18. Summary of Gas Lease Operating Costs and Composite Indices for One Well Producing 1 Million Cubic Feet per Day

\begin{tabular}{|c|c|c|c|c|c|}
\hline \multirow[b]{2}{*}{ Area } & \multicolumn{4}{|c|}{ Index $(1976=100)$} & \multirow{2}{*}{$\begin{array}{c}1995^{*} \\
\text { Cost } \\
\text { (dollars) }\end{array}$} \\
\hline & 1992 & 1993 & 1994 & 1995 & \\
\hline & \multicolumn{5}{|c|}{ 8,000-Foot Wells } \\
\hline South Loulslana............................................... . & 202.3 & 209.9 & 211.5 & 215.3 & 28,200 \\
\hline South Texas............................................. . & 230.7 & 249.6 & 248.8 & 250.4 & 31,800 \\
\hline \multirow[t]{2}{*}{ 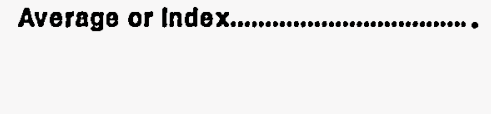 } & 216.3 & 229.5 & 230.2 & 232.6 & 30,000 \\
\hline & \multicolumn{5}{|c|}{ 12,000-Foot Wells } \\
\hline 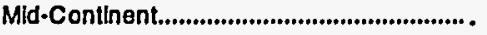 & 239.8 & 250.4 & 247.4 & 247.4 & 32,900 \\
\hline North Loulslana .................................................. & 194.1 & 201.3 & 202.6 & 207.2 & 31,700 \\
\hline South Loulslana................................................. & 204.6 & 211.8 & 213.1 & 217.6 & 33,300 \\
\hline Rocky Mountains............................................... . & 211.5 & 219.9 & 219.9 & 221.2 & 34,500 \\
\hline 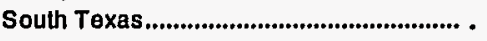 & 191.9 & 209.4 & 208.7 & 211.4 & 31,500 \\
\hline 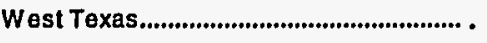 & 222.1 & 221.3 & 218.4 & 218.4 & 29,700 \\
\hline \multirow[t]{2}{*}{ Average or Index...................................... e } & 209.5 & 217.7 & 217.0 & 219.7 & 32,300 \\
\hline & \multicolumn{5}{|c|}{ 16,000-Foot Wells } \\
\hline 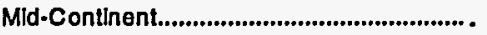 & 233.1 & 243.2 & 239.9 & 240.5 & 35,600 \\
\hline North Loulslana & 196.5 & 202.3 & 204.7 & 208.1 & 35,800 \\
\hline South Loulslana & 198.3 & 204.1 & 206.4 & 210.5 & 36,200 \\
\hline 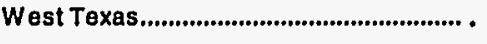 & 222.0 & 221.3 & 218.7 & 220.0 & 33,000 \\
\hline 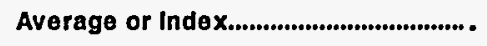 & 210.6 & 216.1 & 216.1 & 218.6 & 35,200 \\
\hline \multicolumn{6}{|l|}{ Aggregate } \\
\hline Average for Production Rate..................... & 212.2 & 219.6 & 219.6 & 222.3 & 32,900 \\
\hline
\end{tabular}

- Preliminary

Note: Reported average or aggtegate average indices are Indlces of the average costs. They are net an average of the Index values.

Source: Energy Information Adminlstration, Ofice of Oll and Gas

Table 19. Summary of Gas Lease Operating Costs and Cómposite Indices for One W ell Producing 5 Million Cubic Feet per Day

\begin{tabular}{|c|c|c|c|c|c|}
\hline \multirow[b]{2}{*}{ Area } & \multicolumn{4}{|c|}{ Index $(1976=100)$} & \multirow{2}{*}{ 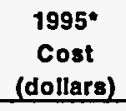 } \\
\hline & 1992 & 1993 & 1994 & 1995 & \\
\hline & \multicolumn{5}{|c|}{ 12,000-Foot Wells } \\
\hline South Loulsiana.............................................. & 195.8 & 203.5 & 204.2 & 209.0 & 30,100 \\
\hline South Texas & 184.9 & 197.6 & 197.0 & 200.0 & 33,200 \\
\hline \multirow[t]{2}{*}{ Average or index...................................... } & 190.3 & 200.6 & 200.6 & 204.5 & 31,700 \\
\hline & \multicolumn{5}{|c|}{ 16,000-Foot Wells } \\
\hline 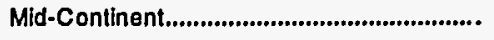 & 181.6 & 188.8 & 186.7 & 188.3 & 36,900 \\
\hline 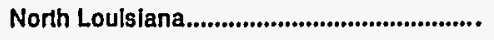 & 177.6 & 184.3 & 185.2 & 189.5 & 39,800 \\
\hline 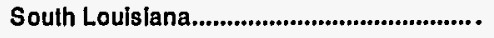 & 177.5 & 183.7 & 185.2 & 189.5 & 39,600 \\
\hline 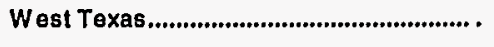 & 179.0 & 182.5 & 180.5 & 182.5 & 36,500 \\
\hline Average or Index.................................. & 178.9 & 184.8 & 184.3 & 187.3 & 38,200 \\
\hline \multicolumn{6}{|l|}{ Aggregate } \\
\hline Average for Production Rate.................. & 181.4 & 188.8 & 188.3 & 191.5 & 36,000 \\
\hline
\end{tabular}

- Preliminary

Note: Reported average or aggregate average indices are Indices of the average costs. They are not an average of the index values.

Source: Energy Information Administration, Office of Oll and Gas

Energy Information Administration 
Table 20. Summary of Gas Lease Operating Costs and Composite Indices for One Well Producing 10 Million Cubic Feet per Day

\begin{tabular}{|c|c|c|c|c|c|}
\hline \multirow[b]{2}{*}{ Area } & \multicolumn{4}{|c|}{ Index $(1976=100)$} & \multirow{2}{*}{$\begin{array}{c}1995^{*} \\
\text { Cost } \\
\text { (dollare) }\end{array}$} \\
\hline & 1992 & 1993 & 1994 & 1995 & \\
\hline & \multicolumn{5}{|c|}{16,000 -Foot Wells } \\
\hline 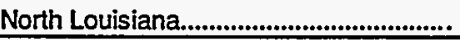 & 170.4 & 176.7 & 179.8 & 182.9 & 52,500 \\
\hline
\end{tabular}

- Preliminary

Note: This is the only area in which a 16,000 -foot well producing 10 MMcf per day is reported.

Source: Energy Information Administration, Office of Oll and Gas

Table 21. Summary of Aggregate Average Gas Lease Operating Cost, by Well Depth (1992-95)

\begin{tabular}{|c|c|c|c|c|}
\hline \multirow{2}{*}{$\begin{array}{c}\text { Well Depth } \\
\text { (feet) }\end{array}$} & \multicolumn{4}{|c|}{ Average Cost, Dollars } \\
\hline & 1992 & 1993 & 1994 & 1995* \\
\hline 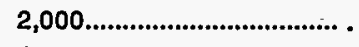 & 9,800 & 10,100 & 10,200 & 10,300 \\
\hline 4,000 & 13,800 & 14,200 & 14,200 & 14,400 \\
\hline $8,000 \ldots \ldots \ldots \ldots$ & 22,700 & 23,500 & 23,500 & 23,700 \\
\hline 12,000 & 28,400 & 29,400 & 29,400 & 29,700 \\
\hline $16,000 \ldots \ldots \ldots \ldots \ldots \ldots \ldots \ldots \ldots \ldots \ldots \ldots \ldots$ & 34,200 & 35,200 & 35,200 & 35,700 \\
\hline
\end{tabular}

- Prellminary

Source: Energy Information Administration, Otfice of Oil and Gas 
Figure 16. Aggregate Average Annual Gas Well Operating Costs for a One-Well Gas Lease by Production Rate, 1992-1995

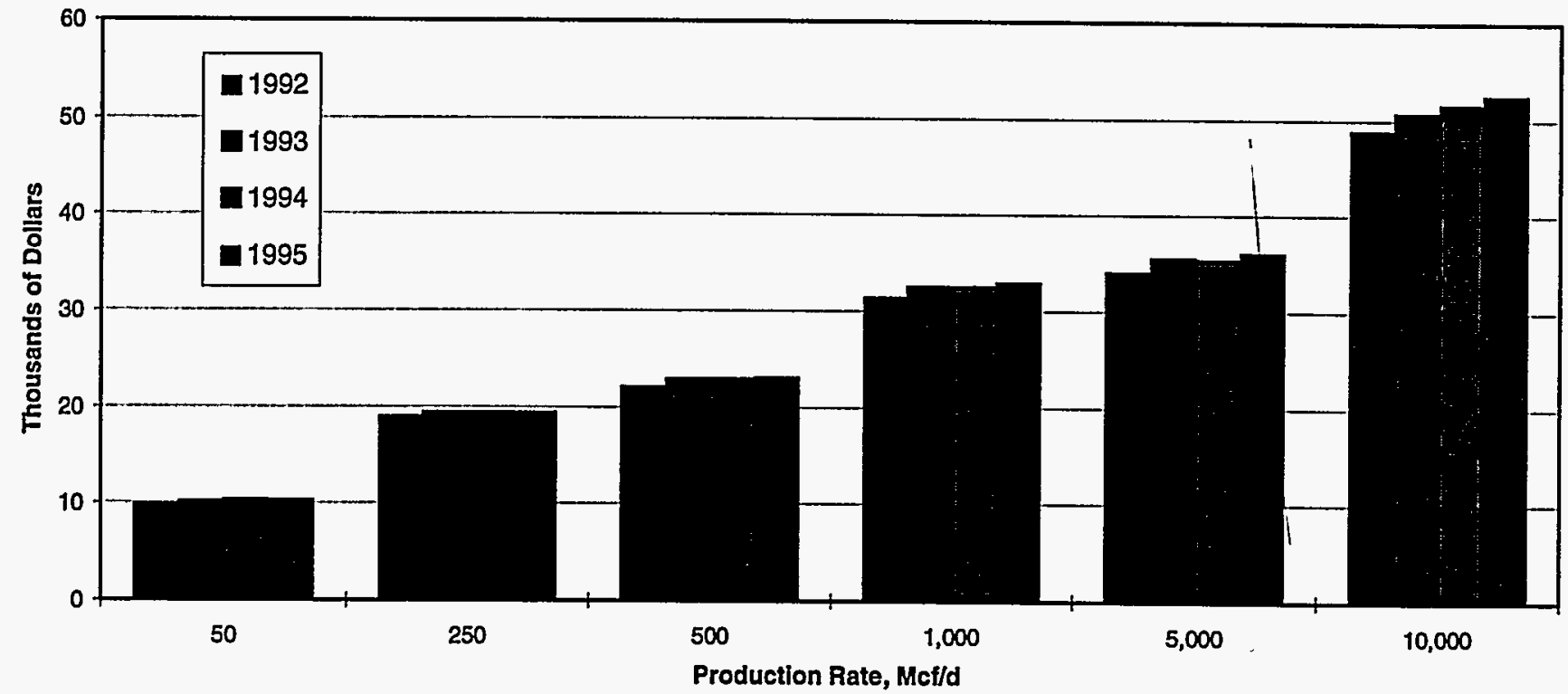

Source: Tables 14 through 19.

Figure 17. Annual Gas Well Operating Costs by Depth and Production Rate, 1995

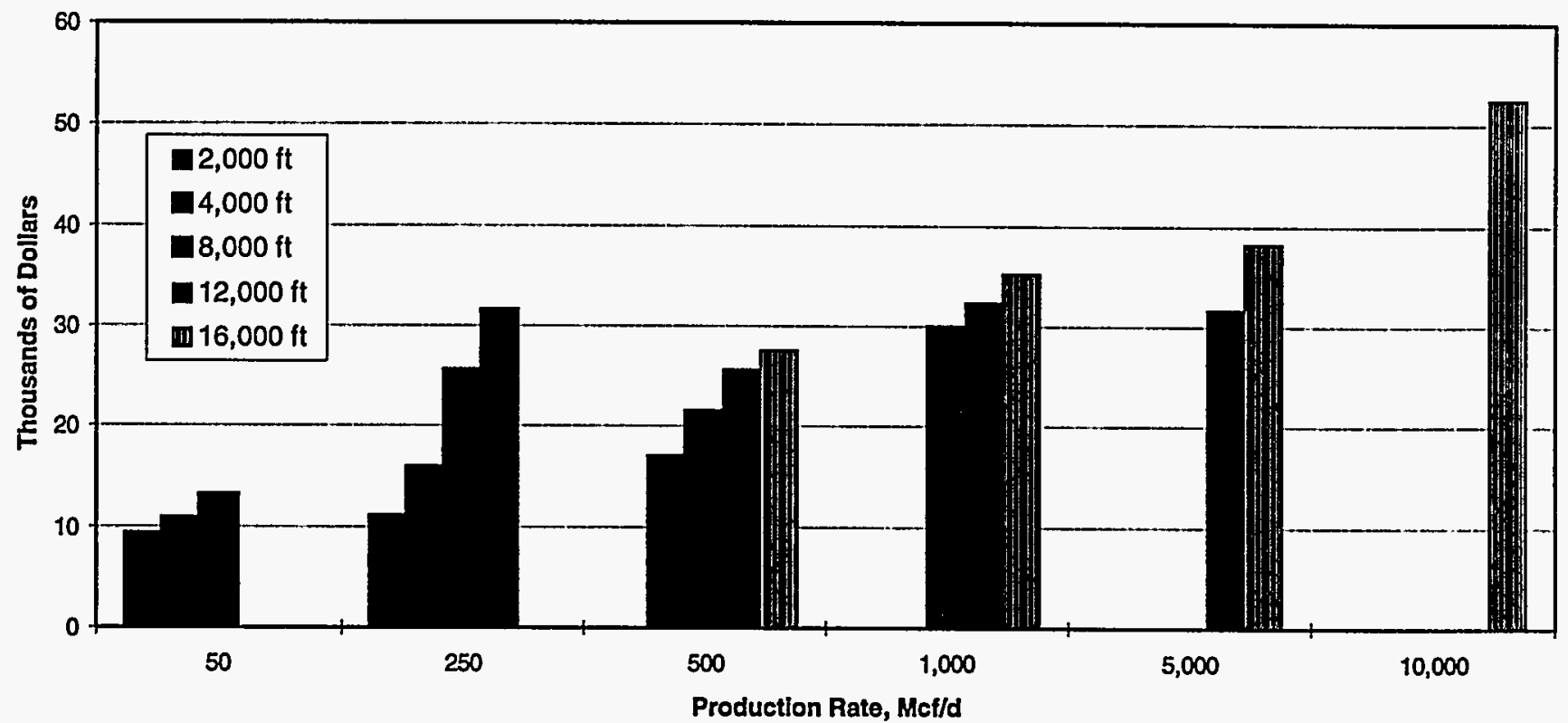

Source: Tables 14 through 19. 


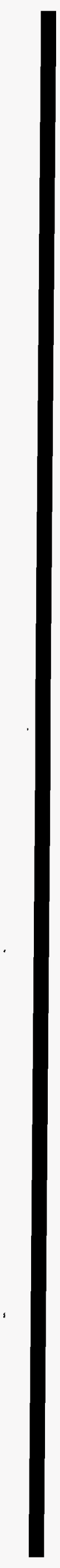




\section{Indexing Review}

\section{Technological and Data Changes}

The uniform oil lease equipment design adopted in 1976 was the basic criterion for oil lease equipment cost estimates. Revisions have been made to stay current with engineering and competitive practices. Individual component prices were combined into one price for a group of equipment, as necessary, to assure confidentiality of prices. Appendix Tables A15 through A18 contain detailed equipment lists of representative wells in west Texas for each depth, reflecting all changes made to date.

Standardization of the data used has evolved during the past 19 years. Improved methods for measuring various contractor costs were used and applied to previous estimates. The gas lease equipment designs were made in 1980 and the equipment and operating components were priced back through 1976. There have been no recent design changes for gas equipment. A typical design is shown in Appendix Table H11, which contains a list of equipment for a 12,000 -foot gas well producing $1 \mathrm{MMcf}$ per day in west Texas.
Estimated preliminary costs for the prior report were revised to reflect new data. Some of these changes and factors were:

- New projections of Joint Association Survey (JAS) data for west Texas were made to estimate 1995 drilling costs.

- $\quad$ Regional wellhead gas prices for 1992-1995 are from the latest edition of the EIA Natural Gas Annual (DOE/EIA-0131 94). These 1995 prices are estimated.

\section{Primary Oil Recovery}

Leases for oil wells were assumed to consist of 10 wells producing by artificial lift into a centrally located tank battery. The depths of all wells on the leases were 2,000 , $4,000,8,000$, or 12,000 feet.

Table 22. Type of Artificial Lift and Prime Mover Used for Each Depth and Region

\begin{tabular}{|c|c|c|c|c|}
\hline Region & $\begin{array}{c}\text { Type of } \\
\text { Lift }\end{array}$ & $\begin{array}{l}\text { Prime } \\
\text { Mover }\end{array}$ & $\begin{array}{c}\text { Type of } \\
\text { Lift }\end{array}$ & $\begin{array}{l}\text { Prime } \\
\text { Mover }\end{array}$ \\
\hline & \multicolumn{2}{|c|}{ 2,000-Foot Wells } & \multicolumn{2}{|c|}{ 4,000-Foot Wells } \\
\hline 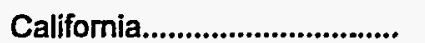 & Rod & Motor & Rod & Motor \\
\hline 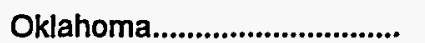 & Rod & Engine & Rod & Engine \\
\hline South Louisiana.................... & Rod & Engine & Gas & Engine \\
\hline 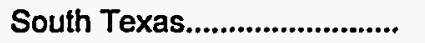 & Rod & Engine & Gas & Engine \\
\hline West Texas.................................... & Rod & Engine & Rod & Engine \\
\hline \multirow[t]{2}{*}{ Rocky Mountains...................... } & Rod & Motor & Rod & Motor \\
\hline & \multicolumn{2}{|c|}{ 8,000-Foot Wells } & \multicolumn{2}{|c|}{ 12,000-Foot Wells } \\
\hline 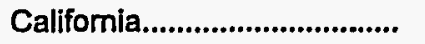 & Hydraulic & Motor & Hydraulic & Motor \\
\hline 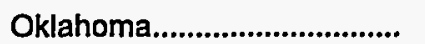 & Hydraulic & Engine & Hydraulic & Engine \\
\hline South Louisiana.................... & Gas & Engine & Hydraulic & Engine \\
\hline South Texas................................... & Gas & Engine & Hydraulic & Engine \\
\hline 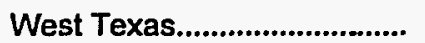 & Rod & Engine & Hydraulic & Engine \\
\hline Rocky Mountains................ & Rod & Motor & Hydraulic & Motor \\
\hline
\end{tabular}

Source: Energy Information Administration, Office of Oil and Gas. 
Costs were determined for new equipment capable of producing 200 barrels of liquid per day per well foronshore primary operations. Tubing costs were included for information only. Note that care must be exercised when combining these equipment costs with drilling costs to obtain total lease development and equipment costs, because most drilling cost estimates include tubing costs. The artificial lift selected was dependent upon the type of lift found to be dominant for each depth in each region. The two types of prime movers considered were electric motors and natural gas engines. Table 22 details the type of lift and prime mover used in each region and depth.Annual operating costs were estimated for daily production rates of 100 barrels of liquid per day per well for each depth in each region of operation.

\section{Secondary Oil Recovery}

Costs for secondary oil recovery in west Texas were calculated for wells producing from depths of 2,000, 4,000 , and 8,000 feet. Each lease had 10 producing wells, 11 injection wells, and 1 disposal well. Additional costs included those for water supply wells, water storage tanks, injection plant, filtering systems, and injection lines. Equipment was designed to handle 350 barrels of liquid per day per producing well. Gas engines used in primary operations were replaced by electric motors for secondary oil recovery. Some equipment for primary oil production was replaced with larger equipment to accommodate the increased liquid volumes assumed for secondary recovery production. Increases in operational costs for secondary oil recovery are indicated for the increased liquid lift of 290 barrels of liquid per day per producing well and the water injection system. Additional equipment costs are presented in Appendix Tables A9, A10, and A11, and direct annual operating costs are presented in Tables A12, A13, and A14.

\section{Offshore Gas and Primary Oil Recovery}

Equipment and operating costs for the offshore Gulf of Mexico were estimated for 12- and 18-slot platforms containing one dually completed well in each slot. Maximum crude oil production was assumed to total 11,000 barrels of oil per day from wells on each platform. Maximum associated gas production was assumed to be 40 MMcf cubic feet of gas per day per platform. Note that the balance between gas and oil is weighted more heavily toward gas in offshore operations than in onshore leases. Operating costs were derived for platforms assumed to be 50,100 , and 125 miles from shore corresponding to water depths of 100,300 , and 600 feet, respectively. Meals, platform maintenance, helicopter and boat transportation of personnel and supplies, communication costs, insurance costs for platform and production equipment and administrative expenses are included in normal production toward gas in offshore operations than in onshore leases. Operating costs were derived for platforms assumed to be 50,100 , and 125 miles from shore corresponding to water depths of 100,300 , and 600 feet, respectively. Meals, platform maintenance, helicopter and boat transportation of personnel and supplies, communication costs, insurance costs for platform and production equipment and administrative expenses are included in normal production expenses. Crude oil and natural gas transportation costs to shore were excluded, as were water disposal costs.

\section{Gas Recovery}

Leases for gas wells were assumed to consist of one well producing into an onsite separator with two storage tanks (a lease condensate sales tank and a water storage tank). Line heaters, dehydration units, and methanol injectors were included where needed. It was assumed that any compression or gas treatment would be provided by the first purchaser. The cost data presented were based on the installation of new equipment and included items needed from the wellhead to the inlet on the meter run for the gas stream and through the tank for the liquid streams. Downhole tubing costs were not included, nor were equipment for disposal of produced water above nominal amounts of water entrained in the gas stream. Gas production rates of 50,250,500,1,000,5,000, and 10,000 Mcf of gas per day and well depths of 2,000, 4,000, $8,000,12,000$, and 16,000 feet were the assumed volume and depth divisions for the cost determinations. These volumes were selected because of different processing equipment requirements for each of these flow rates. Production records were used to determine the average production rate for each depth in each region. The equipment and operating costs for each of these average production rates were then calculated. For a broader view of each flow rate in each region at each depth, the equipment and operating costs of the next higher and/or lower rates are shown. Costs were calculated for equipping gas wells at producing rates of 50 Mcf per day even though a new well coming onstream at this rate may never reach payout. This low rate of flow was selected to identify costs of production from stripper gas wells. Flow rates above $10 \mathrm{MMcf}$ per day usually require custom design of equipment and are not priced in this report.

The depths of $2,000,4,000,8,000$, and 12,000 feet were chosen to be compatible with data provided for oil production. An additional depth of 16,000 feet was added for gas equipment and operations because there was significant gas production from this depth in some regions studied. 


\section{Section I}

\section{Appendices A Through G}

Costs and Indices for Domestic Oil Field

Equipment and Production Operations 


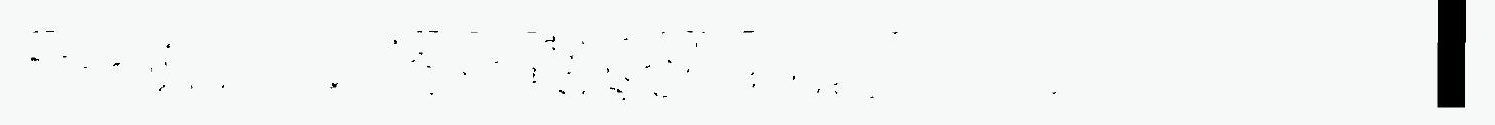




\section{Appendices A Through G}

\section{Costs and Indices for Domestic Oil Field Equipment and Production Operations}

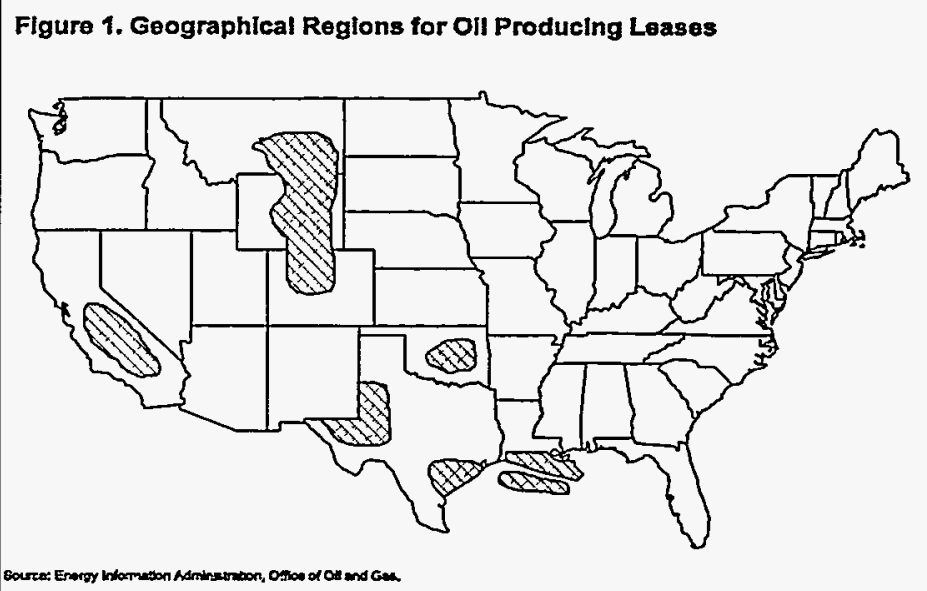

A detailed breakdown of costs and cost indices for 1992 through 1995 is shown in each of the oil lease appendix tables. These tables include both lease equipment costs and direct annual operating costs with their appropriate index numbers. Appendices A through $G$ present the costs and indices for each region and type of operation for oil production.

The tables are arranged by region with each region identified by an alpha character. For example, Tables Al through A18 are for west Texas. Tables A1 through A4 contain equipment costs and indices for primary production for four depths, beginning with the shallowest depth. Tables A5 through A8 are the annual operating costs and indices by depth for primary operations. Tables $\mathrm{A} 9, \mathrm{~A} 10$, and $\mathrm{A} 11$ present additional equipment costs required for secondary operations for three depths.
Tables A12, A13, and A14 contain annual operating costs by depth for secondary production. Tables A15 through A18 are sample detailed equipment listings by depth for the region.

The remaining Tables containing costs and indices for oil leases by region are arranged in similar order. They are: Appendix B--south Texas, Appendix C--south Louisiana, Appendix D--Oklahoma, Appendix E--Wyoming, Appendix F-Califomia, and Appendix G-Gulf of Mexico.

Notes: - 1995 data are preliminary and are marked with a single asterisk $\left(^{*}\right)$. - All prior data were revised. Indices marked with a double asterisk $\left({ }^{* *}\right)$ are composite indices. - Other indices are pure cost. - Entries with (***) are estimated from Joint Association Survey on Drilling Costs data. 
Table A1. Lease Equipment Costs and Indices for Primary Oil Production in West Texas (10 Wells Producing from 2,000 Feet by Rod Lift)

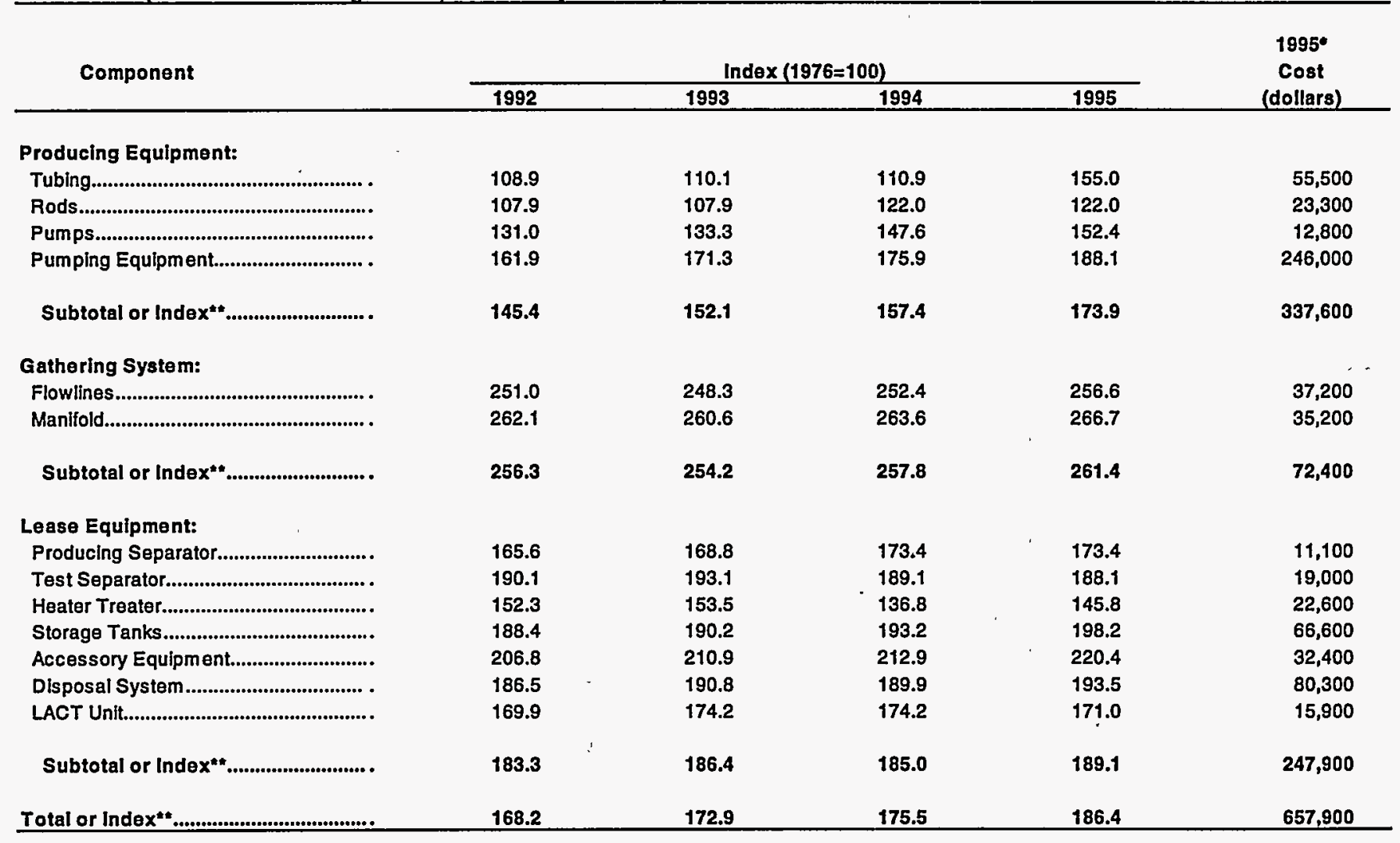

Table A2. Lease Equipment Costs and Indices for Primary Oil Production In West Texas (10 Wells Producing from 4,000 Feet by Rod Lift)

\begin{tabular}{|c|c|c|c|c|c|c|}
\hline \multirow[t]{2}{*}{ Component } & & \multicolumn{4}{|c|}{ Index $(1976=100)$} & \multirow{2}{*}{$\begin{array}{c}1995^{\circ} \\
\text { Cost } \\
\text { (dollars) }\end{array}$} \\
\hline & & 1992 & 1993 & 1994 & 1995 & \\
\hline \multicolumn{7}{|l|}{ Producing Equipment: } \\
\hline 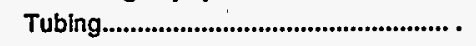 & & 107.4 & 108.7 & 109.2 & 152.9 & 111,000 \\
\hline 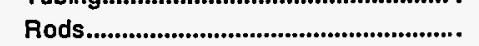 & & 101.5 & 101.5 & 113.3 & 113.6 & 44,400 \\
\hline 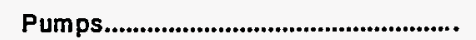 & & 132.9 & 136.6 & 134.1 & 154.9 & 12,700 \\
\hline Pumping Equipment.................................... . & & 146.3 & 155.7 & .158 .4 & 177.6 & 398,200 \\
\hline 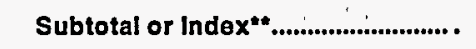 & & 132.7 & 139.2 & 142.3 & 164.6 & 566,300 \\
\hline \multicolumn{7}{|l|}{ Gathering System: } \\
\hline 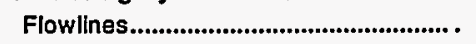 & & 239.4 & 236.7 & 240.4 & 244.1 & 45,900 \\
\hline 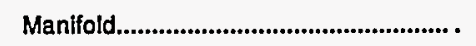 & & 262.1 & 260.6 & 263.6 & 266.7 & 35,200 \\
\hline Subtotal or Index $x^{*} \ldots \ldots \ldots \ldots \ldots \ldots \ldots$ & & 248.8 & 246.6 & 250.0 & 253.4 & 81,100 \\
\hline \multicolumn{7}{|l|}{ Lease Equipment: } \\
\hline 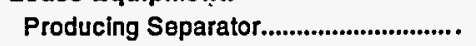 & & 165.6 & 168.8 & 173.4 & 173.4 & 11,100 \\
\hline 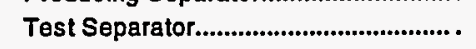 & & 190.1 & 193.1 & 189.1 & 188.1 & 19,000 \\
\hline 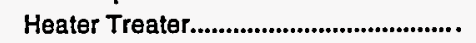 & . & 152.3 & 153.5 & 136.8 & 145.8 & 22,600 \\
\hline 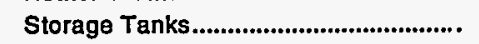 & & 188.4 & 190.2 & 193.2 & 198.2 & 66,600 \\
\hline 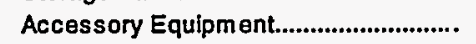 & & 206.8 & 210.9 & 212.9 & 220.4 & 32,400 \\
\hline 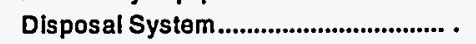 & & 185.7 & 190.4 & 189.5 & 193.0 & 82,600 \\
\hline 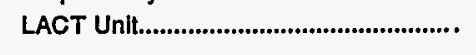 & & 169.9 & 174.2 & 174.2 & 171.0 & 15,900 \\
\hline 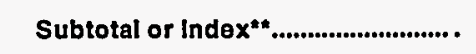 & & 183.1 & 186.3 & 185.0 & 189.0 & 250,200 \\
\hline 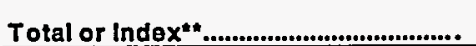 & & 153.1 & 158.2 & 160.2 & 176.5 & 897,600 \\
\hline
\end{tabular}


Table A3. Lease Equipment Costs and Indices for Primary Oll Production in West Texas (10 Wells Producing from 8,000 Feet by Rod Litt)

\begin{tabular}{|c|c|c|c|c|c|}
\hline \multirow[t]{2}{*}{ Component } & \multicolumn{4}{|c|}{ Index $(1976=100)$} & \multirow{2}{*}{$\begin{array}{c}1995^{\circ} \\
\text { Cost } \\
\text { (dollars) }\end{array}$} \\
\hline & 1992 & 1993 & 1994 & 1995 & \\
\hline \multicolumn{6}{|l|}{ Producing Equipment: } \\
\hline 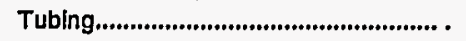 & 110.5 & 109.3 & 111.5 & 147.6 & 276,500 \\
\hline 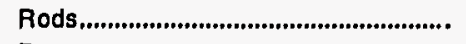 & 89.7 & 89.8 & 100.1 & 100.1 & 100,000 \\
\hline 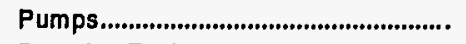 & 136.7 & 136.7 & 157.8 & 172.2 & 15,500 \\
\hline Pumping Equipment................................. . & 154.3 & 165.7 & 173.0 & 190.2 & 808,300 \\
\hline Subtotal or Index ${ }^{4+\ldots} \ldots$ & 133.8 & 140.2 & 146.7 & 166.5 & $1,200,300$ \\
\hline \multicolumn{6}{|l|}{ Gathering System: } \\
\hline 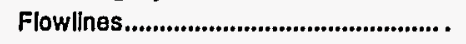 & 228.2 & 225.1 & 228.6 & 232.4 & 60,200 \\
\hline 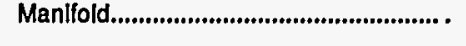 & 262.1 & 260.6 & 263.6 & 266.7 & 35,200 \\
\hline Subtotal or Index & 239.6 & 237.1 & 240.4 & 244.0 & 95,400 \\
\hline \multicolumn{6}{|l|}{ Lease Equipment: } \\
\hline Producing Separator...................................... & 165.6 & 168.8 & 173.4 & 173.4 & 11,100 \\
\hline 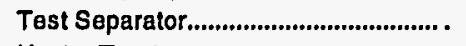 & 190.1 & 193.1 & 189.1 & 188.1 & 19,000 \\
\hline 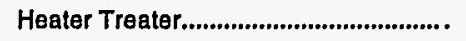 & 152.3 & 153.5 & 136.8 & 145.8 & 22,600 \\
\hline 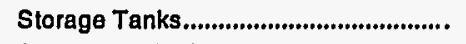 & 188.4 & 190.2 & 193.2 & 198.2 & 66,600 \\
\hline 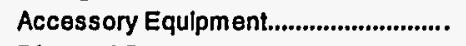 & 206.8 & 210.9 & 212.9 & 220.4 & 32,400 \\
\hline 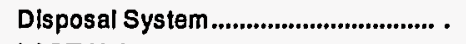 & 199.2 & 204.6 & 203.5 & 207.5 & 77,400 \\
\hline 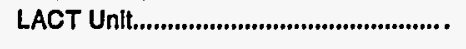 & 169.9 & 174.2 & 174.2 & 171.0 & 15,900 \\
\hline Subtotal or Index & 186.9 & 190.3 & 188.9 & 193.1 & 245,000 \\
\hline 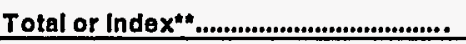 & 146.0 & 151.6 & 156.9 & 173.7 & $1,540,700$ \\
\hline
\end{tabular}

Table A4. Lease Equipment Costs and Indices for Primary Oil Production in West Texas (10 Wells Producing from 12,000 Feet by Hydraulic Lift)

\begin{tabular}{|c|c|c|c|c|c|}
\hline \multirow[t]{2}{*}{ Component } & \multicolumn{4}{|c|}{ Index $(1976=100)$} & \multirow{2}{*}{$\begin{array}{c}\text { 1995* } \\
\text { Cost } \\
\text { (dollars) }\end{array}$} \\
\hline & 1992 & 1993 & 1994 & 1995 & \\
\hline \multicolumn{6}{|l|}{ Producing Equipment: } \\
\hline 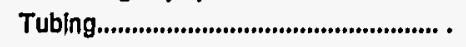 & 206.5 & 162.6 & 136.6 & 144.1 & 736,000 \\
\hline 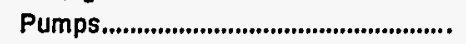 & 153.4 & 153.4 & 250.5 & 261.7 & 196,000 \\
\hline Pumplng Equipment.............................. . & 192.7 & 189.8 & 171.7 & 176.5 & 313,900 \\
\hline Subtotal or Index & 198.1 & 168.1 & 155.9 & 163.2 & $1,245,900$ \\
\hline \multicolumn{6}{|l|}{ Gathering System: } \\
\hline 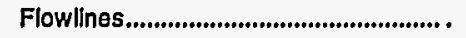 & 248.0 & 213.6 & 190.3 & 192.9 & 174,800 \\
\hline Manifold......................................................... . & 262.1 & 260.6 & 263.6 & 266.7 & 35,200 \\
\hline Subtotal or Index & 249.8 & 219.6 & 199.6 & 202.3 & 210,000 \\
\hline \multicolumn{6}{|l|}{ Lease Equipment: } \\
\hline Producing Separator................................ & 165.6 & 168.8 & 173.4 & 173.4 & 11,100 \\
\hline Test Separator........................................ . & 190.1 & 193.1 & 189.1 & 188.1 & 19,000 \\
\hline Heater Treater............................................ & 152.3 & 153.5 & 136.8 & 145.8 & 22,600 \\
\hline Storage Tanks ...................................... & 188.4 & 190.2 & 193.2 & 198.2 & 66,600 \\
\hline Accessory Equipment........................... & 206.8 & 210.9 & 212.9 & 220.4 & 32,400 \\
\hline 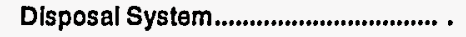 & 199.2 & 204.6 & 203.5 & 207.5 & 77,400 \\
\hline 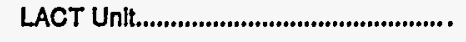 & 169.9 & 174.2 & 174.2 & 171.0 & 15,900 \\
\hline Subtotal or Index & 186.9 & 190.3 & 188.9 & 193.1 & 245,000 \\
\hline Total or Index*"... & 202.0 & 176.3 & 164.7 & 171.1 & $1,700,900$ \\
\hline
\end{tabular}

Energy Information Administration 
Table A5. Direct Annual Operating Costs and Indices for Primary Oil Production in West Texas (10 Wells Producing from 2,000 Feet by Rod Lift)

\begin{tabular}{|c|c|c|c|c|c|}
\hline \multirow[t]{2}{*}{ Component } & \multicolumn{4}{|c|}{ Index $(1976=100)$} & \multirow{2}{*}{$\begin{array}{c}\text { 1995* } \\
\text { Cost } \\
\text { (dollars) }\end{array}$} \\
\hline & 1992 & 1993 & 1994 & 1995 & \\
\hline \multicolumn{6}{|l|}{ Normal Daily Expense: } \\
\hline 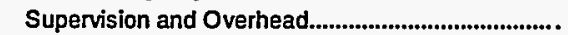 & 305.0 & 315.0 & 315.0 & 325.0 & 19,500 \\
\hline 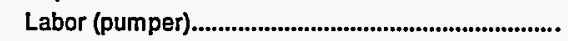 & 263.5 & 255.4 & 255.4 & 255.4 & 18,900 \\
\hline 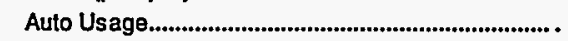 & 257.7 & 257.7 & 261.5 & 273.1 & 7,100 \\
\hline 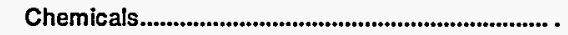 & 203.7 & 203.7 & 203.7 & 203.7 & 5,500 \\
\hline 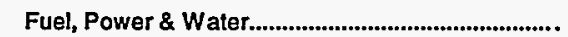 & 233.3 & 270.8 & 266.7 & 244.4 & 17,600 \\
\hline 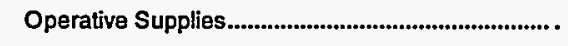 & 233.3 & 233.3 & 233.3 & 233.3 & 1,400 \\
\hline 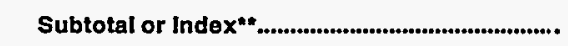 & 257.4 & 267.5 & 266.8 & 264.2 & 70,000 \\
\hline \multicolumn{6}{|l|}{ Surface Maintenance, Repair \& Services: } \\
\hline 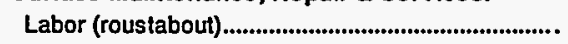 & 237.9 & 231.0 & 231.0 & 231.0 & 6,700 \\
\hline Supplies \& Services........................................................... & 234.4 & 231.3 & 228.1 & 231.3 & 7,400 \\
\hline 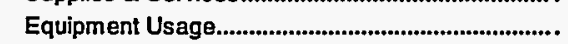 & 246.2 & 253.8 & 253.8 & 253.8 & 3,300 \\
\hline 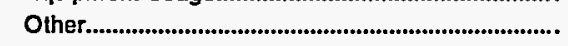 & 180.0 & 180.0 & 173.3 & 173.3 & 2,600 \\
\hline 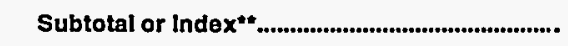 & 228.1 & 225.8 & 223.6 & 224.7 & 20,000 \\
\hline \multicolumn{6}{|l|}{ Subsurface Maintenance, Repair \& Services: } \\
\hline 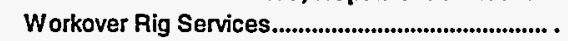 & 217.9 & 217.9 & 215.4 & 215.4 & 8,400 \\
\hline Remedial Services....................................................... & 131.3 & 137.5 & 137.5 & 137.5 & 2,200 \\
\hline 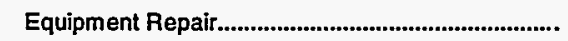 & 128.9 & 131.1 & 144.4 & 151.1 & 6,800 \\
\hline 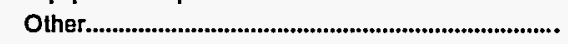 & 150.0 & 150.0 & 200.0 & 200.0 & 400 \\
\hline Subtotal or Index ${ }^{* *} \ldots$ & 163.7 & 165.7 & 171.6 & 174.5 & 17,800 \\
\hline Tatal or Index ${ }^{* *} \ldots \ldots \ldots \ldots$ & 230.7 & 236.6 & 237.1 & 236.4 & 107,800 \\
\hline
\end{tabular}

Table A6. Direct Annual Operating Costs and Indices for Primary Oil Production in West Texas (10 Wells Producing from 4,000 Feet by Rod Lift)

\begin{tabular}{|c|c|c|c|c|c|}
\hline \multirow[t]{2}{*}{ Component } & \multicolumn{4}{|c|}{ Index $(1976=100)$} & \multirow{2}{*}{$\begin{array}{c}\text { 1995* } \\
\text { Cost } \\
\text { (dollars) } \\
\end{array}$} \\
\hline & 1992 & 1993 & 1994 & 1995 & \\
\hline \multicolumn{6}{|l|}{ Normal Daily Expense: } \\
\hline Supervision and Overhead...................................... & 302.9 & 314.7 & 314.7 & 323.5 & 22,000 \\
\hline Labor (pumper) & 263.5 & 255.4 & 255.4 & 255.4 & 18,900 \\
\hline 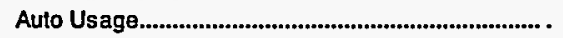 & 257.7 & 257.7 & 261.5 & 273.1 & 7,100 \\
\hline 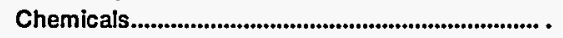 & 218.5 & 218.5 & 218.5 & 218.5 & 5,900 \\
\hline Fuel, Power \& Water............................................... & 236.4 & 276.1 & 265.9 & 243.2 & 21,400 \\
\hline 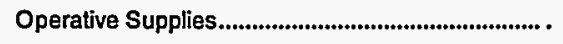 & 250.0 & 250.0 & 250.0 & 250.0 & 1,500 \\
\hline Subtotal or Index & 259.5 & 272.3 & 269.6 & 265.7 & 76,800 \\
\hline \multicolumn{6}{|l|}{ Surface Maintenance, Repair \& Services: } \\
\hline Labor (roustabout) & 237.9 & 231.0 & 231.0 & 231.0 & 6,700 \\
\hline 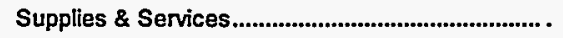 & 235.3 & 232.4 & 232.4 & 232.4 & 7,900 \\
\hline 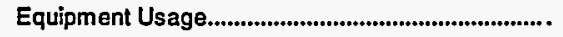 & 253.8 & 261.5 & 261.5 & 261.5 & 3,400 \\
\hline 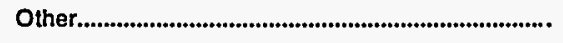 & 187.5 & 187.5 & 183.3 & 183.3 & 4,400 \\
\hline Subtotal or Index & 227.0 & 225.0 & 224.0 & 224.0 & 22,400 \\
\hline \multicolumn{6}{|l|}{ Subsurface Maintenance, Repair \& Services: } \\
\hline Workover Rig Services............................................ . & 215.2 & 215.2 & 212.1 & 212.1 & 14,000 \\
\hline 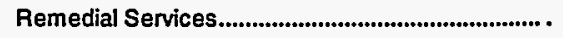 & 156.5 & 160.9 & 160.9 & 160.9 & 3,700 \\
\hline 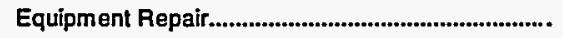 & 124.5 & 126.5 & 128.6 & 149.0 & 7,300 \\
\hline 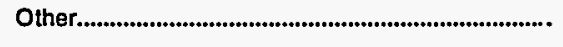 & 166.7 & 166.7 & 166.7 & 166.7 & 500 \\
\hline Subtotal or Index & 173.0 & 174.5 & 173.8 & 180.9 & 25,500 \\
\hline Total or Index ${ }^{* 4}$ & 230.4 & 237.4 & 235.5 & 235.3 & 124,700 \\
\hline
\end{tabular}


Table A7. Direct Annual Operating Costs and Indices for Primary Oil Production in West Texas (10 Wells Producing from 8,000 Feet by Rod Lift)

\begin{tabular}{|c|c|c|c|c|c|}
\hline \multirow[t]{2}{*}{ Component } & \multicolumn{4}{|c|}{ Index $(1976=100)$} & \multirow{2}{*}{$\begin{array}{c}\text { 1995* } \\
\text { Cost } \\
\text { (dollars) }\end{array}$} \\
\hline & 1992 & 1993 & 1994 & 1995 & \\
\hline \multicolumn{6}{|l|}{ Normal Dally Expense: } \\
\hline 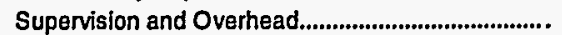 & 306.3 & 317.7 & 317.7 & 326.6 & 25,800 \\
\hline Labor (pumper) & 263.5 & 255.4 & 255.4 & 255.4 & 18,900 \\
\hline 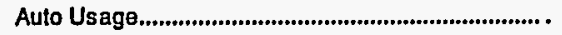 & 257.7 & 257.7 & 261.5 & 273.1 & 7,100 \\
\hline 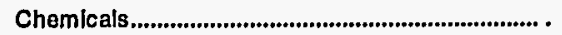 & 200.0 & 200.0 & 200.0 & 196.6 & 5,700 \\
\hline 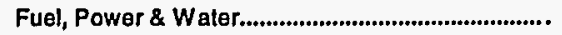 & 239.8 & 281.3 & 264.1 & 239.8 & 30,700 \\
\hline Operative Supplies............................................................. & 228.6 & 228.6 & 228.6 & 228.6 & 1,600 \\
\hline Subtotal or Index & 258.0 & 274.3 & 268.2 & 261.8 & 89,800 \\
\hline \multicolumn{6}{|l|}{ Surface Maintenance, Repair \& Services: } \\
\hline 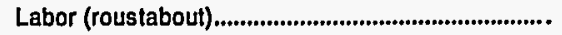 & 237.9 & 231.0 & 231.0 & 231.0 & 6,700 \\
\hline 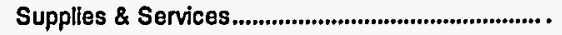 & 230.8 & 228.2 & 228.2 & 228.2 & 8,900 \\
\hline 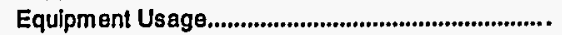 & 213.3 & 220.0 & 220.0 & 220.0 & 3,300 \\
\hline 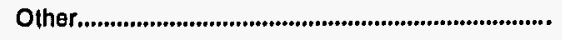 & 180.0 & 180.0 & 176.7 & 176.7 & 5,300 \\
\hline 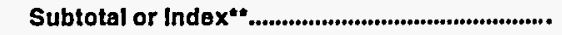 & 216.8 & 215.0 & 214.2 & 214.2 & 24,200 \\
\hline \multicolumn{6}{|l|}{ Subsurface Maintenance, Repair \& Servlces: } \\
\hline Workover Rig Services................................................... . & 236.8 & 236.8 & 234.7 & 234.7 & 33,800 \\
\hline Remedial Services............................................................. & 190.0 & 190.0 & 185.7 & 187.1 & 13,100 \\
\hline 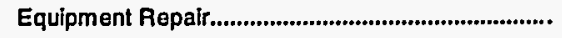 & 148.3 & 146.7 & 163.3 & 183.3 & 11,000 \\
\hline Other........., & 166.7 & 166.7 & 166.7 & 177.8 & 1,600 \\
\hline Subtotal or Index & 204.2 & 203.9 & 205.3 & 210.2 & 59,500 \\
\hline 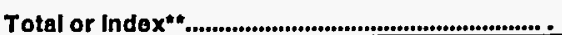 & 231.1 & 238.3 & 235.9 & 234.8 & 173,500 \\
\hline
\end{tabular}

Table A8. Direct Annual Operating Costs and Indices for Primary Oil Production in West Texas (10 Wells Producing from 12,000 Feet by Hydraulic Lift)

\begin{tabular}{|c|c|c|c|c|c|}
\hline \multirow[t]{2}{*}{ Component } & \multicolumn{4}{|c|}{ Index $(1976=100)$} & \multirow{2}{*}{$\begin{array}{c}\text { 1995* } \\
\text { Cost } \\
\text { (dollars) }\end{array}$} \\
\hline & 1992 & 1993 & 1994 & 1995 & \\
\hline \multicolumn{6}{|l|}{ Normal Daily Expense: } \\
\hline 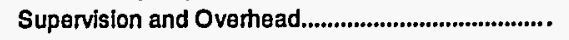 & 307.1 & 319.4 & 318.4 & 327.6 & 32,100 \\
\hline Labor (pumper) & 263.5 & 255.4 & 255.4 & 255.4 & 18,900 \\
\hline 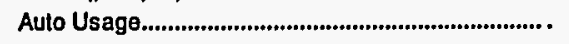 & 257.7 & 257.7 & 261.5 & 273.1 & 7,100 \\
\hline 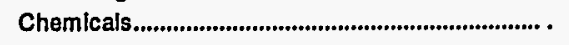 & 190.9 & 190.9 & 190.9 & 190.9 & 6,300 \\
\hline Fuel, Power \& Water................................................... & 240.3 & 283.0 & 261.4 & 236.4 & 41,600 \\
\hline 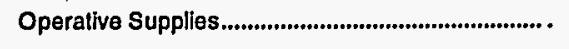 & 290.0 & 300.0 & 290.0 & 290.0 & 2,900 \\
\hline Subtotal or Index*".................................................... & 258.5 & 278.2 & 268.8 & 261.2 & 108,900 \\
\hline \multicolumn{6}{|l|}{ Surface Malntenance, Repalr \& Services: } \\
\hline 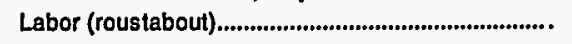 & 237.9 & 231.0 & 231.0 & 231.0 & 6,700 \\
\hline 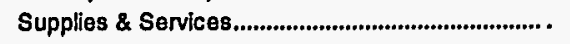 & 221.8 & 227.7 & 222.8 & 223.8 & 22,600 \\
\hline Equipment Usage,................................................... & 213.3 & 220.0 & 220.0 & 220.0 & 3,300 \\
\hline 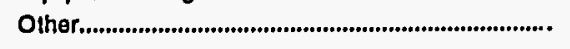 & 216.7 & 216.7 & 216.7 & 216.7 & 1,300 \\
\hline Subtotal or Index & 223.8 & 227.2 & 223.8 & 224.5 & 33,900 \\
\hline \multicolumn{6}{|l|}{ Subsurface Malntenance, Repair \& Services: } \\
\hline 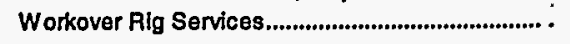 & 206.1 & 206.1 & 198.5 & 198.5 & 13,100 \\
\hline Remedial Services....................................................... & 195.8 & 196.6 & 192.4 & 195.0 & 23,200 \\
\hline 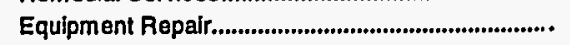 & 249.7 & 250.3 & 403.9 & 421.8 & 75,500 \\
\hline 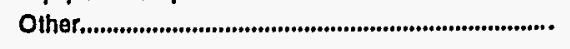 & 166.7 & 166.7 & 175.0 & 175.0 & 2,100 \\
\hline Subtotal or Index & 222.3 & 222.9 & 293.6 & 302.9 & 113,900 \\
\hline Total or Index*\#..... & 238.6 & 248.0 & 271.5 & 271.9 & 256.700 \\
\hline
\end{tabular}


Table A9. Additional Lease Equipment Costs and Indices for Secondary Oil Production in West Texas (10 Wells Producing from 2,000 Feet by Rod Lift and 11 Water injection Wells)

\begin{tabular}{|c|c|c|c|c|c|c|}
\hline \multirow[t]{2}{*}{ Component } & \multicolumn{5}{|c|}{ Index $(1976=100)$} & \multirow{2}{*}{$\begin{array}{c}1995^{\star} \\
\text { Cost } \\
\text { (dollars) }\end{array}$} \\
\hline & 1992 & & 1993 & 1994 & 1995 & \\
\hline \multicolumn{7}{|l|}{ Injection Equipment: } \\
\hline 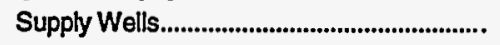 & 170.3 & & 163.9 & 175.0 & 179.6 & 131,300 \\
\hline 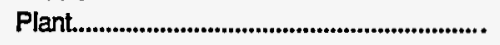 & 291.4 & & 296.7 & 290.2 & 299.4 & 100,900 \\
\hline Distribution Lines.............................................. & 169.7 & & 183.4 & 180.6 & 178.4 & 75,300 \\
\hline 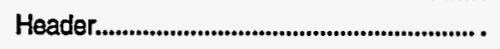 & 254.1 & & 253.6 & 258.5 & 263.8 & 54,600 \\
\hline Electrical Service.............................................. & 328.3 & & 328.7 & 327.2 & 330.6 & 87,600 \\
\hline Subtotal or Index & 221.2 & & 222.6 & 225.3 & 229.2 & 449,700 \\
\hline \multicolumn{7}{|l|}{ ProducIng Equipment: } \\
\hline Tubing Replacement....................................... . & 120.2 & - & 121.4 & 120.4 & 143.1 & 56,100 \\
\hline 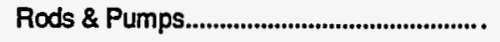 & 110.1 & & 110.7 & 119.8 & 121.1 & 38,500 \\
\hline Pumping Equipment........................................ & 258.2 & ‘ & 274.5 & 281.6 & 335.7 & 32,900 \\
\hline Subtotal or Index & 132.9 & & 135.8 & 139.7 & 157.8 & 127,500 \\
\hline \multicolumn{7}{|l|}{ Injectlon Wells:*t* } \\
\hline Subtotal or Index & 147.1 & . & 143.3 & 138.3 & 137.0 & 725,600 \\
\hline Total or IndeX & 216.3 & & 213.8 & 210.9 & 213.4 & $1,302,800$ \\
\hline
\end{tabular}

Table A10. Additional Lease Equipment Costs and indices for Secondary Oil Production in West Texas (10 Wells Producing from 4,000 Feet by Rod Lift and 11 Water injection Wells)

\begin{tabular}{|c|c|c|c|c|c|}
\hline \multirow[t]{2}{*}{ Component } & \multicolumn{4}{|c|}{ Index $(1976=100)$} & \multirow{2}{*}{$\begin{array}{c}\text { 1995* } \\
\text { Cost } \\
\text { (dollars) }\end{array}$} \\
\hline & 1992 & 1993 & 1994 & 1995 & \\
\hline \multicolumn{6}{|l|}{ Injection Equipment: } \\
\hline 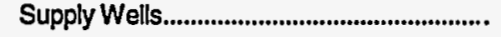 & 168.7 & 162.1 & 173.2 & 177.8 & 130,000 \\
\hline 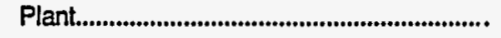 & 287.3 & 292.9 & 286.4 & 295.6 & 99,900 \\
\hline Distribution Lines................................................. & 170.1 & 183.9 & 180.6 & 178.4 & 75,300 \\
\hline 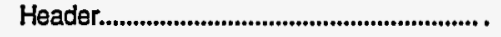 & 254.1 & 253.6 & 258.5 & 263.8 & 54,600 \\
\hline 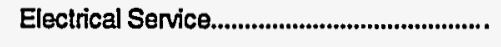 & 238.9 & 239.1 & 238.0 & 240.5 & 88,500 \\
\hline Subtotal or Index & 209.4 & 210.8 & 213.3 & 217.0 & $448,300^{\circ}$ \\
\hline \multicolumn{6}{|l|}{ Producing Equipment: } \\
\hline Tubing Replacement....................................... & 116.4 & 117.6 & 117.2 & 158.7 & 121,100 \\
\hline 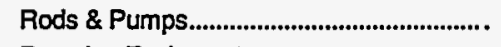 & 103.6 & 104.0 & 112.9 & 113.9 & 59,900 \\
\hline Pumping Equipment..................................... . & 135.5 & 141.0 & 141.4 & 162.5 & 326,100 \\
\hline Subtotal or Index & 126.0 & 129.6 & 131.2 & 153.9 & 507,100 \\
\hline \multicolumn{6}{|l|}{ Injection Welis:**t } \\
\hline Subtotal or Index & 153.3 & 157.1 & 152.8 & 151.3 & $1,717,600$ \\
\hline Total or Index & 154.8 & 158.3 & 156.0 & 159.9 & $2,673,000$ \\
\hline
\end{tabular}


Table A11. Additional Lease Equipment Costs and Indices for Secondary Oil Production in West Texas (10 Wells Producing from 8,000 Feet by Rod Lift and 11 Water Injection Wells)

\begin{tabular}{|c|c|c|c|c|c|}
\hline \multirow[t]{2}{*}{ Component } & \multicolumn{4}{|c|}{ Index $(1976=100)$} & \multirow{2}{*}{$\begin{array}{c}1995^{*} \\
\text { Cost } \\
\text { (dollar8) }\end{array}$} \\
\hline & 1992 & 1993 & 1994 & 1995 & \\
\hline \multicolumn{6}{|l|}{ Injectlon Equipment: } \\
\hline 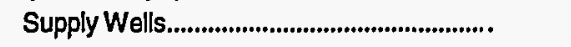 & 169.1 & 161.2 & 174.0 & 178.6 & 231,800 \\
\hline 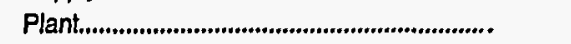 & 301.7 & 307.4 & 302.4 & 311.8 & 205,800 \\
\hline Distribution Lines.................................................. & 170.5 & 184.4 & 181.1 & 178.9 & 113,400 \\
\hline 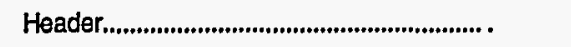 & 234.1 & 233.0 & 237.5 & 241.9 & 64,600 \\
\hline 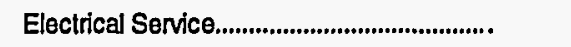 & 255.1 & 254.8 & 252.5 & 253.5 & 135,600 \\
\hline Subtotal or Index ${ }^{*+\ldots}$ & 213.8 & 214.4 & 217.6 & 221.3 & 751,200 \\
\hline \multicolumn{6}{|l|}{ Producing Equipment: } \\
\hline 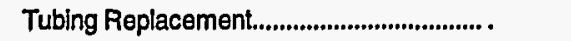 & 115.7 & 114.6 & 116.1 & 151.1 & 291,600 \\
\hline 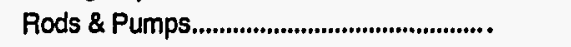 & 93.0 & 93.0 & 102.3 & 101.5 & 120,300 \\
\hline Pumping Equipment.......................................... & 135.5 & 142.6 & 143.1 & 161.6 & 607,800 \\
\hline Subtotal or Index $x^{* *} \ldots$ & 122.6 & 126.2 & 128.5 & 148.3 & $1,019,700$ \\
\hline \multicolumn{6}{|l|}{ Injectlon Wells:*** } \\
\hline Subtotal or Index $x^{* *}, \ldots \ldots \ldots \ldots \ldots$ & 133.3 & 127.4 & 118.3 & 117.1 & $3,490,400$ \\
\hline Total or Indox & 138.3 & 134.6 & 128.4 & 131.3 & $5,261,300$ \\
\hline \multirow[t]{2}{*}{ Component } & \multicolumn{4}{|c|}{ Index $(1976=100)$} & $\begin{array}{l}\text { 1995" } \\
\text { Cost }\end{array}$ \\
\hline & 1992 & 1993 & 1994 & 1995 & (dollars) \\
\hline \multicolumn{6}{|l|}{ Normal Dally Expense: } \\
\hline 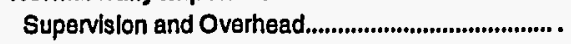 & 309.0 & 317.2 & 318.6 & 329.0 & 47,700 \\
\hline 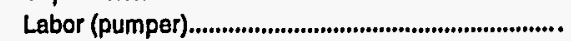 & 304.2 & 295.0 & 295.0 & 295.0 & 35,400 \\
\hline Chemicals......................................................................... & 202.9 & 202.9 & 202.9 & 200.0 & 7,000 \\
\hline Fuel, Power \& Water......................................................... & 212.6 & 216.6 & 259.9 & 257.0 & 77,600 \\
\hline Operative Supplies.............................................................. . & 280.0 & 266.7 & 273.3 & 273.3 & 4,100 \\
\hline Subtotal or Index & 254.1 & 255.9 & 277.6 & 278.4 & 171,800 \\
\hline \multicolumn{6}{|l|}{ Surface Maintenance, Repalr \& Services: } \\
\hline 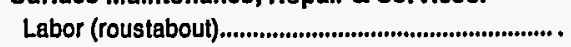 & 239.1 & 231.9 & 231.9 & 231.9 & 16,000 \\
\hline 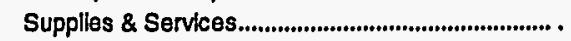 & 227.3 & 221.8 & 227.3 & 228.2 & 25,100 \\
\hline 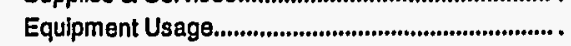 & 278.3 & 282.6 & 282.6 & 282.6 & 6,500 \\
\hline Other & 193.3 & 193.3 & 186.7 & 186.7 & 2,800 \\
\hline Subtotal or Index & 234.1 & 229.5 & 231.8 & 232.3 & 50,400 \\
\hline \multicolumn{6}{|l|}{ Subsurface Maintenance, Repalt \& Servlces: } \\
\hline Workover Rig Services................................................... & 227.3 & 227.3 & 222.4 & 222.4 & 31,800 \\
\hline Remedlal Services........................................................... & 178.0 & 180.5 & 178.0 & 180.5 & 7,400 \\
\hline 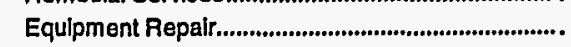 & 139.6 & 139.6 & 137.7 & 137.7 & 7,300 \\
\hline Other................, & 182.9 & 188.6 & $\begin{array}{c}191.4 \\
\cdot\end{array}$ & 194.3 & 6,800 \\
\hline Subtotal or Index & 197.1 & 198.2 & 195.2 & 196.0 & 53,300 \\
\hline Total or Inde $x^{* *} \ldots$ & 236.2 & 236.5 & 248.4 & 249.1 & 275,500 \\
\hline
\end{tabular}


Table A13. Direct Annual Operating Costs and indices for Secondary Oll Production in West Texas (10 Wells Producing from 4,000 Feet by Rod Lift and 11 Water Injection Wells)

\begin{tabular}{|c|c|c|c|c|c|}
\hline \multirow[t]{2}{*}{ Component } & \multicolumn{4}{|c|}{ Index $(1976=100)$} & \multirow{2}{*}{$\begin{array}{c}\text { 1995" } \\
\text { Cost } \\
\text { (dollars) }\end{array}$} \\
\hline & 1992 & 1993 & 1994 & 1995 & \\
\hline \multicolumn{6}{|l|}{ Normal Daily Expense: } \\
\hline 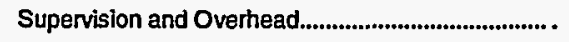 & 309.0 & 317.2 & 318.6 & 329.0 & 47,700 \\
\hline 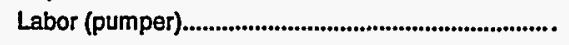 & 303.9 & 295.0 & 295.0 & 295.0 & 53,100 \\
\hline 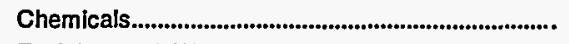 & 209.4 & 209.4 & 209.4 & 209.4 & 6,700 \\
\hline Fuel, Power \& Water.................................................... & 212.6 & 215.5 & 255.5 & 253.1 & 94,400 \\
\hline Operative Supplies......................................................... . & 250.0 & 242.3 & 250.0 & 250.0 & 6,500 \\
\hline 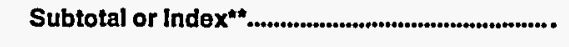 & 314.2 & 315.1 & 340.1 & 341.1 & 208,400 \\
\hline \multicolumn{6}{|l|}{ Surface Maintenance, Repair \& Services: } \\
\hline 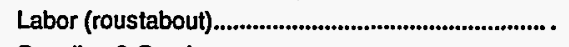 & 241.2 & 234.2 & 234.2 & 234.2 & 26,700 \\
\hline Supplies \& Services........................................................ . & 220.5 & 214.6 & 221.1 & 222.2 & 38,000 \\
\hline 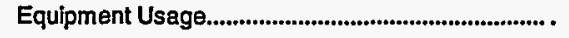 & 308.7 & 313.0 & 313.0 & 313.0 & 14,400 \\
\hline 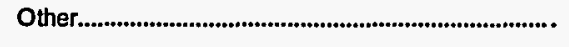 & 200.0 & 200.0 & 191.7 & 191.7 & 2,300 \\
\hline Subtotal or Index & 238.5 & 233.8 & 236.7 & 237.3 & 81,400 \\
\hline \multicolumn{6}{|l|}{ Subsurface Maintenance, Repair \& Services: } \\
\hline Workover Rig Services............................................. & 222.2 & 222.2 & 217.9 & 217.9 & 45,100 \\
\hline 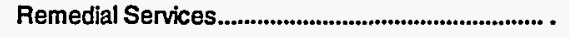 & 189.7 & 191.2 & 188.2 & 191.2 & 13,000 \\
\hline 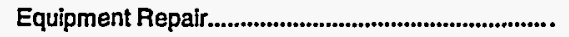 & 133.3 & 133.3 & 131.0 & 131.0 & 11,000 \\
\hline 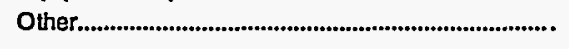 & 177.6 & 181.6 & 185.7 & 189.8 & 9,300 \\
\hline Subtotal or Index & 193.1 & 193.9 & 191.2 & 192.2 & 78,400 \\
\hline Total or Index & 258.9 & 258.3 & 269.5 & 270.3 & 368,200 \\
\hline
\end{tabular}

Table A14. Direct Annual Operating Costs and Indices for Secondary Oll Production in West Texas (10 Wells Producing from 8,000 Feet by Rod Lift and 11 Water Injection Wells)

\begin{tabular}{|c|c|c|c|c|c|}
\hline \multirow[t]{2}{*}{ Component } & \multicolumn{4}{|c|}{ Index $(1976=100)$} & \multirow{2}{*}{$\begin{array}{c}1995^{\star} \\
\text { Cost } \\
\text { (dollars) }\end{array}$} \\
\hline & 1992 & 1993 & 1994 & 1995 & \\
\hline \multicolumn{6}{|l|}{ Normal Daily Expense: } \\
\hline Supervision and Overhead......................................... & 305.3 & 316.6 & 316.0 & 324.9 & 54,900 \\
\hline Labor (pumper) & 303.9 & 295.0 & 295.0 & 295.0 & 53,100 \\
\hline 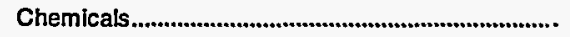 & 209.4 & 209.4 & 209.4 & 209.4 & 6,700 \\
\hline 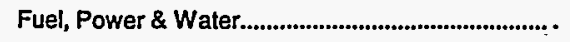 & 230.3 & 231.7 & 282.3 & 280.9 & 180,600 \\
\hline 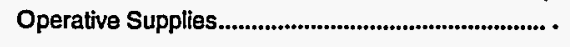 & 253.8 & 246.2 & 250.0 & 250.0 & 6,500 \\
\hline Subtotal or Index $x^{* \star}$ & 303.9 & 305.0 & 341.9 & 342.6 & 301,800 \\
\hline \multicolumn{6}{|l|}{ Surface Maintenance, Repair \& Services: } \\
\hline Labor (roustabout) & 241.2 & 234.2 & 234.2 & 234.2 & 26,700 \\
\hline 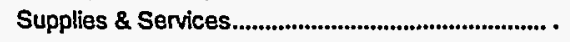 & 230.5 & 223.4 & 230.5 & 230.5 & 38,500 \\
\hline Equipment Usage....................................................... & 264.0 & 270.8 & 270.8 & 270.8 & 24,100 \\
\hline 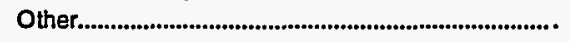 & 190.0 & 190.0 & 180.0 & 180.0 & 1,800 \\
\hline Subtotal or Index & 240.5 & 236.8 & 239.7 & 239.7 & . 91,100 \\
\hline \multicolumn{6}{|l|}{ Subsurface Maintenance, Repair \& Services: } \\
\hline Workover Rig Services..................................................... & 215.5 & 215.5 & 211.8 & 211.8 & 86,000 \\
\hline 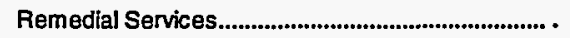 & 246.3 & 246.3 & 240.3 & 242.5 & 32,500 \\
\hline Equipment Repair.......................................................... & 120.9 & 120.9 & 119.4 & 119.4 & 16,600 \\
\hline 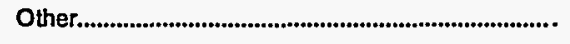 & 173.3 & 177.3 & 181.3 & 185.3 & 13,900 \\
\hline Subtotal or index & 199.3 & 199.7 & 196.8 & 197.6 & 149,000 \\
\hline Total or Index & 252.8 & 252.8 & 268.3 & 268.9 & 541,900 \\
\hline
\end{tabular}


Table A15. Detailed Lease Equipment List for 2,000-Foot Wells in West Texas (10 Producing Wells)

Tubing $(20,000$ feet $)$

Size: $2-3 / 8$ inches

Weight: 4.7 pounds per foot

Grade: $\mathrm{H}-40$

\section{Sucker Rods (20,000 feet)}

Size: $5 / 8$ inches

API class: $\mathrm{K}$

Rod Pump (10)

API type: TH

Size: 2 by $1-3 / 4$ inches by 9 feet

Pumping Unit (10)

API size: C57D-76-54

Engine: 9 horsepower single cylinder

\section{Oil Flowline $(11,500$ feet $)$}

Size: 2-3/8 inches

Material: polyvinyl chloride 1120

Weight: 0.43 pounds per foot

Pressure rating: 160 pounds per square inch

Manifold (1)

Valves: 2-inch, 3-way, 2-position, electric operated (10)

Production Separator (1)

Type: vertical

Size: 30 inches by 10 feet

Capacity: 2,700 barrels of fluid per day and 5.7 million

cubic feet of gas per day

Vapor Recovery Unit (1)

Capacity: 40 thousand cubic feet of gas per day
Test Separator (1)

Type: vertical

Size: 24 inches by 7-1/2 feet

Capacity: 1.290 barrels of fluid per day

Working pressure: 125 pounds per square inch

Net oil computer: Electronic

Heater Treater (1)

Working pressure: 50 pounds per square inch

Size: 4 feet by $27-1 / 2$ feet

Oil Storage Tanks (2)

Storage capacity: 2,000 barrels

Type: 10-gauge, bolted steel

Construction: gas tight

Size: 30 feet by 16 feet

Water Disposal Pump (1)

Type: Quintuplex

Plungers: $1-1 / 2$ inches

Working pressure: 1,000 pounds per square inch

Electric motor: 20 horsepower

Water Disposal Line (2,000 feet)

Size: 2-3/8 inches

Weight: 3.75 pounds per foot

Grade: B

Mill test: 2,500 pounds per square inch

LACT Unit (1)

Capacity: 2,000 barrels per day

Working pressure: 125 pounds per square inch

Source: Energy Information Administration, Office of Oil and Gas. 


\section{Tubing (40,000 feet)}

Size: $2-3 / 8$ inches

Weight: 4.7 pounds per foot

Grade: J-55

\section{Sucker Rods (20,000 feet)}

Size: $5 / 8$ inches $(24,000$ feet)

Size: $3 / 4$ inches $(16,000$ feet)

API class: $\mathrm{K}$

Rod Pump (10)

API type: RWBC

Size: 2 by $1-1 / 2$ inches by 9 feet

Pumping Unit (10)

API size: M160D-173-74

Engine: 12 horsepower single cylinder

Oil Flowline $(16,000$ feet $)$

Size: 2-3/8 inches

Material: polyvinyl chloride 1120

Weight: 0.43 pounds per foot

Pressure rating: 160 pounds per square inch

Manifold (1)

Valves: 2-inch, 3-way, 2-position, electric operated (10)

Production Separator (1)

Type: vertical

Size: 30 inches by 10 feet

Capacity: 2,700 barrels of fluid per day and 5.7 million

cubic feet of gas per day

Vapor Recovery Unit (1)

Capacity: 40 thousand cubic feet of gas per day

\section{Test Separator (1)}

Type: vertical

Size: 24 inches by 7-1/2 feet

Capacity: 1.290 barrels of fluid per day

Working pressure: 125 pounds per square inch

Net oil computer: Electronic

Heater Treater (1)

Working pressure: 50 pounds per square inch

Size: 4 feet by $27-1 / 2$ feet

Oil Storage Tanks (2)

Storage capacity: 2,000 barrels

Type: 10-gauge, bolted steel

Construction: gas tight

Size: 30 feet by 16 feet

Water Disposal Pump (1)

Type: Quintuplex

Plungers: $1-1 / 2$ inches

Working pressure: 1,000 pounds per square inch

Electric motor: 20 horsepower

\section{Water Disposal Line (2,000 feet)}

Size: 2-3/8 inches

Weight: 3.75 pounds per foot

Grade: B

Mill test: 2,500 pounds per square inch

\section{LACT Unit (1)}

Capacity: 2,000 barrels per day

Working pressure: 125 pounds per square inch

Source: Energy Information Administration, Office of Oil and Gas. 
Tubing $(80,000$ feet $)$

Size: 2-7/8 inches

Weight: 6.5 pounds per foot

Grade: J-55

\section{Sucker Rods $(80,000$ feet $)$}

Size: 1 inch $(15,250$ feet)

Size: $7 / 8$ inches $(17,500$ feet)

Size: $3 / 4$ inches $(47,250$ feet $)$

API class: $\mathrm{K}$

Rod Pump (10)

API type: RWBC

Size: $2-1 / 2$ by $1-1 / 4$ inches by 20 feet

Pumping Unit (10)

API size: M456D-305-144

Engine: 32 horsepower single cylinder

Oll Flowline $(23,200$ feet $)$

Size: 2-3/8 inches

Material: polyvinyl chloride 1120

Weight: 0.43 pounds per foot

Pressure rating: 160 pounds per square inch

Manifold (1)

Valves: 2-inch, 3-way, 2-position, electric operated (10)

Production Separator (1)

Type: vertical

Size: 30 inches by 10 feet

Capacity: 2,700 barrels of fluid per day and 5.7 million

cubic feet of gas per day

Vapor Recovery Unit (1)

Capacity: 40 thousand cubic feet of gas per day
Test Separator (1)

Type: vertical

Size: 24 inches by $7-1 / 2$ feet

Capacity: 1.290 barrels of fluid per day

Working pressure: 125 pounds per square inch

Net oil computer: Electronic

Heater Treater (1)

Working pressure: 50 pounds per square inch

Size: 4 feet by $27-1 / 2$ feet

Oll Storage Tanks (2)

Storage capacity: 2,000 barrels

Type: 10-gauge, bolted steel

Construction: gas tight

Size: 30 feet by 16 feet

Water Disposal Pump (1)

Type: Quintuplex

Plungers: $1-1 / 2$ inches

Working pressure: 1,000 pounds per square inch

Electric motor: 20 horsepower

Water Disposal Line $(3,400$ feet)

Size: 2-3/8 inches

Weight: 3.75 pounds per foot

Grade: B

Mill test: 2,500 pounds per square inch

LACT Unit (1)

Capacity: 2,000 barrels per day

Working pressure: 125 pounds per square inch

Source: Energy Information Administration, Office of Oil and Gas. 
Tubing (240,000 feet)

Size: $2-7 / 8$ inches $(120,000$ feet)

Weight: 6.5 pounds per foot

Grade: N-80

Size: 1.66 inches (120,000 feet)

Weight: 2.4 pounds per foot

Grade: J-55

Hydraulic Bottom Hole Pump (10)

Size: 2 by $1-3 / 8$ by $1-3 / 16$ inches

\section{Surface Pumping Equipment (4)}

Type: Triplex

Engine: 6-cylinder, 100 horsepower

Power Oil Flowlines $(23,200$ feet $)$

Size: $1-1 / 2$ inches

Grade: J-55

Power Oil Tank (1)

Storage capacity: 750 barrels

Type: 10-gauge, bolted steel

Construction: gas tight

Size: $15-1 / 2$ feet by 24 feet

Oil Flowlines (23,200 feet)

Size: 2-3/8 inches

Material: polyvinyl chloride 1120

Weight: 0.43 pounds per foot

Pressure rating: 160 pounds per square inch

Manifold (1)

Valves: 2-inch, 3-way, 2-position, electric operated (10)

Production Separator (1)

Type: vertical

Size: 30 inches by 10 feet

Capacity: 2,700 barrels of fluid per day and 5.7 million

cubic feet of gas per day
Test Separator (1)

Type: vertical

Size: 24 inches by $7-1 / 2$ feet

Capacity: 1.290 barrels of fluid per day

Working pressure: 125 pounds per square inch

Net oil computer: Electronic

Heater Treater (1)

Working pressure: 50 pounds per square inch

Size: 4 feet by $27-1 / 2$ feet

Oil Storage Tanks (2)

Storage capacity: 2,000 barrels

Type: 10-gauge, bolted steel

Construction: gas tight

Size: 30 feet by 16 feet

Water Disposal Pump (1)

Type: Quintuplex

Plungers: $1-1 / 2$ inches

Working pressure: 1,000 pounds per square inch

Electric motor: 20 horsepower

Water Disposal Line ( 2,000 feet)

Size: $2-3 / 8$ inches

Weight: 3.75 pounds per foot

Grade: B

Mill test: 2,500 pounds per square inch

LACT Unit (1)

Capacity: 2,000 barrels per day

Working pressure: 125 pounds per square inch

Vapor Recovery Unit (1)

Capacity: $\mathbf{4 0}$ thousand cubic feet of gas per day

Source: Energy Information Administration, Office of Oil and Gas. 
Table B1. Lease Equipment Costs and Indices for Primary Oll Production in South Texas (10 Wells Producing from 2,000 Feet by Rod Lift)

\begin{tabular}{|c|c|c|c|c|c|}
\hline \multirow{2}{*}{ Component } & \multicolumn{4}{|c|}{ Index $(1976=100)$} & \multirow{2}{*}{$\begin{array}{c}1995^{*} \\
\text { Cost } \\
\text { (dollars) }\end{array}$} \\
\hline & 1992 & 1993 & 1994 & 1995 & \\
\hline \multicolumn{6}{|l|}{ Producing Equipment: } \\
\hline 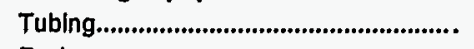 & 109.2 & 110.6 & 111.2 & 155.5 & 55,500 \\
\hline 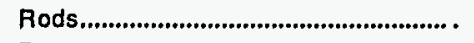 & 101.0 & 102.0 & 118.9 & 122.4 & 24,600 \\
\hline 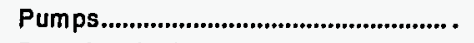 & 131.0 & 133.3 & 147.6 & 152.4 & 12,800 \\
\hline Pumping Equlpment.................................. & 162.2 & 172.6 & 177.3 & 189.4 & 247,200 \\
\hline Subtotal or lndex ${ }^{* *} \ldots \ldots \ldots . . . . . . . . . . . . . . . . . . . .$. & 144.8 & 152.3 & 157.9 & 174.7 & 340,100 \\
\hline \multicolumn{6}{|l|}{ Gathering System: } \\
\hline 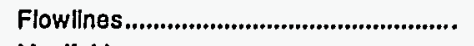 & 284.6 & 305.4 & 309.4 & 313.4 & 46,700 \\
\hline 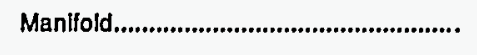 & 261.2 & 260.4 & 263.4 & 266.4 & 35,700 \\
\hline Subtotal or Index"*................................. & 273.5 & 284.1 & 287.6 & 291.2 & 82,400 \\
\hline \multicolumn{6}{|l|}{ Lease Equipment: } \\
\hline Producing Separator................................... . & 165.6 & 168.8 & 173.4 & 173.4 & 11,100 \\
\hline 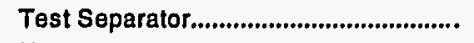 & 190.1 & 193.1 & 189.1 & 188.1 & 19,000 \\
\hline Heater Treater............................................. . & 152.3 & 153.5 & 136.8 & 145.8 & 22,600 \\
\hline 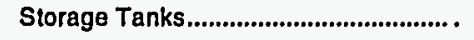 & 190.1 & 195.8 & 198.8 & 203.9 & 68,300 \\
\hline Accessory Equipm ent.............................. & 206.8 & 214.3 & 216.3 & 223.8 & 32,900 \\
\hline 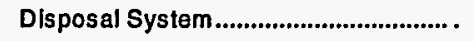 & 183.2 & 191.8 & 192.3 & 196.7 & 84,400 \\
\hline LACT Unit................................................... . & 169.9 & 174.2 & 174.2 & 171.0 & 15,900 \\
\hline Subtotal or Inde $x^{* *}$ & 182.7 & 188.6 & 187.7 & 192.0 & 254,200 \\
\hline 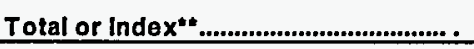 & 269.6 & 281.0 & 285.8 & 303.5 & 676,700 \\
\hline
\end{tabular}

Table B2. Lease Equipment Costs and Indices for Primary Oil Production in South Texas (10 Wells Producing from 4,000 Feet by Gas Lift)

\begin{tabular}{|c|c|c|c|c|c|}
\hline \multirow{2}{*}{ Component } & \multicolumn{4}{|c|}{ Index $(1976=100)$} & \multirow{2}{*}{$\begin{array}{c}\text { 1995. } \\
\text { Cost } \\
\text { (dollars) }\end{array}$} \\
\hline & 1992 & 1993 & 1994 & 1995 & \\
\hline \multicolumn{6}{|l|}{ Producing Equipment: } \\
\hline 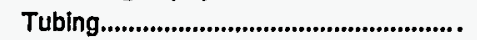 & 105.7 & 106.9 & 107.5 & 151.1 & 109,400 \\
\hline 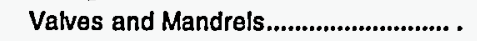 & 342.7 & 349.8 & 390.1 & 394.5 & 99,800 \\
\hline Pumping Equipment................................... . & 160.1 & 162.9 & 163.9 & 169.9 & 177,400 \\
\hline 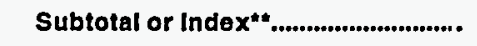 & 163.4 & 166.3 & 172.0 & 191.3 & 386,600 \\
\hline \multicolumn{6}{|l|}{ Gathering System: } \\
\hline 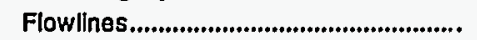 & 160.4 & 168.8 & 184.1 & 186.0 & 160,500 \\
\hline 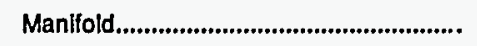 & 261.2 & 260.4 & 263.4 & 266.4 & 35,700 \\
\hline Subtotal or Index $x^{*} \ldots \ldots \ldots \ldots \ldots \ldots$ & 173.9 & 181.1 & 194.8 & 196.8 & 196,200 \\
\hline \multicolumn{6}{|l|}{ Lease Equipment: } \\
\hline Producing Separator............................. . & 165.6 & 168.8 & 173.4 & 173.4 & 11,100 \\
\hline 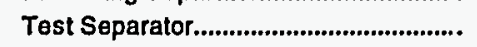 & 190.1 & 193.1 & 189.1 & 188.1 & 19,000 \\
\hline Heater Treater......................................... . & 152.3 & 153.5 & 136.8 & 145.8 & 22,600 \\
\hline 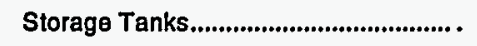 & 190.1 & 195.8 & 198.8 & 203.9 & 68,300 \\
\hline 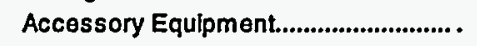 & 206.8 & 214.3 & 216.3 & 223.8 & 32,900 \\
\hline 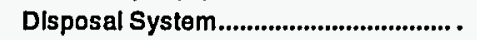 & 182.6 & 191.9 & 192.1 & 196.4 & 87,000 \\
\hline 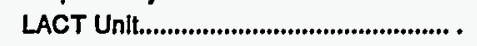 & 169.9 & 174.2 & 174.2 & 171.0 & 15,900 \\
\hline Subtotal or Index" & 182.5 & 188.6 & 187.7 & 191.9 & 256,800 \\
\hline 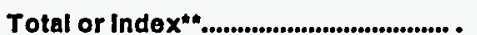 & 171.7 & 176.5 & 182.0 & 192.7 & 839,600 \\
\hline
\end{tabular}


Table B3. Lease Equipment Costs and Indices for Primary Oll Production in South Texas (10 Wells Producing from 8,000 Feet by Gas Lift)

\begin{tabular}{|c|c|c|c|c|c|}
\hline \multirow{2}{*}{ Component } & \multicolumn{4}{|c|}{ Index $(1976=100)$} & \multirow{2}{*}{$\begin{array}{c}\text { 1995* } \\
\text { Cost } \\
\text { (dollars) }\end{array}$} \\
\hline & 1992 & 1993 & 1994 & 1995 & \\
\hline \multicolumn{6}{|l|}{ Producing Equipment: } \\
\hline 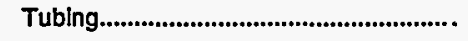 & 105.8 & 107.1 & 107.6 & 151.4 & 218,900 \\
\hline Valves and Mandrels................................. . & 342.9 & 350.0 & 390.4 & 394.9 & 139,800 \\
\hline 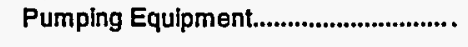 & 158.4 & 161.2 & 162.2 & 168.2 & 177,400 \\
\hline Subtotal or Index $x^{*}$ & 154.6 & 157.2 & 162.8 & 187.8 & 536,100 \\
\hline \multicolumn{6}{|l|}{ Gathering System: } \\
\hline 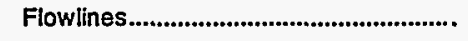 & 156.4 & 164.8 & 180.2 & 182.6 & 247,200 \\
\hline 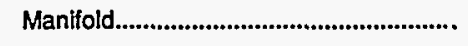 & 261.2 & 260.4 & 263.4 & 266.4 & 35,700 \\
\hline Subtotal or Index & 165.9 & 173.5 & 187.7 & 190.1 & 282,900 \\
\hline \multicolumn{6}{|l|}{ Lease Equipment: } \\
\hline Producing Separator................................... . & 165.6 & 168.8 & 173.4 & 173.4 & 11,100 \\
\hline Test Separator................................................ & 190.1 & 193.1 & 189.1 & 188.1 & 19,000 \\
\hline Heater Treater......................................... . & 152.3 & 153.5 & 136.8 & 145.8 & 22,600 \\
\hline 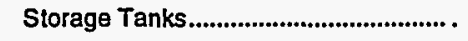 & 190.1 & 195.8 & 198.8 & 203.9 & 68,300 \\
\hline 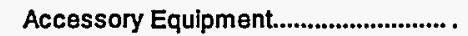 & 206.8 & 214.3 & 216.3 & 223.8 & 32,900 \\
\hline Disposal System & 183.1 & 190.2 & 191.2 & 195.6 & 80,000 \\
\hline 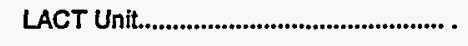 & 169.9 & 174.2 & 174.2 & 171.0 & 15,900 \\
\hline Subtotal or Index & 182.7 & 188.0 & 187.3 & 191.6 & 249,800 \\
\hline 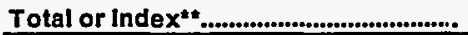 & 164.1 & 168.6 & 175.0 & 189.3 & $1,068,800$ \\
\hline
\end{tabular}

Table B4. Lease Equipment Costs and Indices for Primary Oil Production in South Texas (10 Wells Producing from 12,000 Feet by Hydraulic Lift)

\begin{tabular}{|c|c|c|c|c|c|}
\hline \multirow{2}{*}{ Component } & \multicolumn{4}{|c|}{ Index $(1976=100)$} & \multirow{2}{*}{$\begin{array}{c}\text { 1995* } \\
\text { Cast } \\
\text { (dollars) } \\
\end{array}$} \\
\hline & 1992 & 1993 & 1994 & 1995 & \\
\hline \multicolumn{6}{|l|}{ Producing Equipment: } \\
\hline 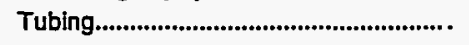 & 207.2 & 163.2 & 137.0 & 144.6 & 736,000 \\
\hline 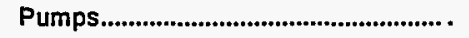 & 153.4 & 153.4 & 250.5 & 261.7 & 196,000 \\
\hline Pumping Equipment.............................. . & 192.8 & 196.9 & 178.7 & 183.6 & 326,500 \\
\hline Subtotal or Index $x^{* * \ldots . . . . . . . . . . . . . . . . . . . . . . . . . ~}$ & 198.5 & 170.1 & 157.9 & 165.2 & $1,258,500$ \\
\hline \multicolumn{6}{|l|}{ Gathering System: } \\
\hline 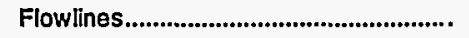 & 239.0 & 224.4 & 214.9 & 216.7 & 236,800 \\
\hline 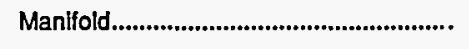 & 261.2 & 260.4 & 263.4 & 266.4 & 35,700 \\
\hline Subtotal or Index $x^{* n}$ & 239.0 & 224.4 & 214.9 & 216.7 & 236,800 \\
\hline \multicolumn{6}{|l|}{ Lease Equipment: } \\
\hline Producing Separator............................... . & 165.6 & 168.8 & 173.4 & 173.4 & 11,100 \\
\hline Test Separator........................................ & 190.1 & 193.1 & 189.1 & 188.1 & 19,000 \\
\hline Heater Treater....................................... . & 152.3 & 153.5 & 136.8 & 145.8 & 22,600 \\
\hline 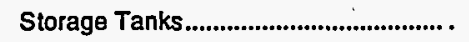 & 190.1 & 195.8 & 198.8 & 203.9 & 68,300 \\
\hline Accessory Equipment............................. . & 206.8 & 214.3 & 216.3 & 223.8 & 32,900 \\
\hline Disposal System ..................................... . & 182.9 & 190.6 & 193.2 & 197.8 & 81,900 \\
\hline 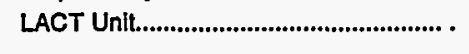 & 169.9 & 174.2 & 174.2 & 171.0 & 15,900 \\
\hline Subtotal or Index & 182.6 & 188.2 & 187.9 & 192.3 & 251,700 \\
\hline Total or Index ${ }^{* *}$ & 200.8 & 178.4 & 168.1 & 174.4 & $1,747,000$ \\
\hline
\end{tabular}


Table B5. Direct Annual Operating Costs and Indices for Primary Oil Production in South Texas (10 Wells Producing from 2,000 Feet by Rod Litt)

\begin{tabular}{|c|c|c|c|c|c|}
\hline \multirow[t]{2}{*}{ Component } & \multicolumn{4}{|c|}{ Index $(1976=100)$} & \multirow{2}{*}{$\begin{array}{c}\text { 1995* } \\
\text { Cost } \\
\text { (dollars) }\end{array}$} \\
\hline & 1992 & 1993 & 1994 & 1995 & \\
\hline \multicolumn{6}{|l|}{ Normal Dally Expenso: } \\
\hline 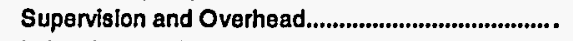 & 305.0 & 316.7 & $\mathbf{3 1 5 . 0}$ & 325.0 & 19,500 \\
\hline 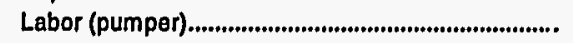 & 274.4 & 317.4 & 317.4 & 317.4 & 27,300 \\
\hline 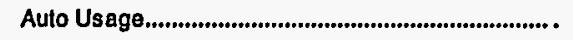 & 268.0 & 268.0 & 272.0 & 284.0 & 7,100 \\
\hline 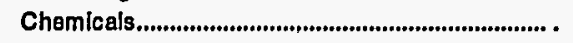 & 207.4 & 211.1 & 211.1 & 207.4 & 5,600 \\
\hline Fuel, Power \& Water.......................................................: & 246.6 & 286.3 & 258.9 & 232.9 & 17,000 \\
\hline Operative Supplies........................................................... & 218.2 & 254.5 & 254.5 & 254.5 & 2,800 \\
\hline Subtotal or Index & 264.5 & 292.2 & 285.1 & 281.2 & 79,300 \\
\hline \multicolumn{6}{|l|}{ Surface Maintenance, Repalr \& Sorvices: } \\
\hline Labor (roustabout) & 187.3 & 221.1 & 221.1 & 221.1 & 15,700 \\
\hline 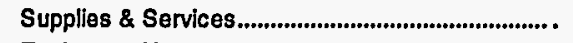 & 189.1 & 218.2 & 218.2 & 218.2 & 12,000 \\
\hline 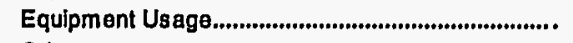 & 217.4 & 239.1 & 239.1 & 239.1 & 5,500 \\
\hline Other & 275.0 & 316.7 & 316.7 & 316.7 & 3,800 \\
\hline Subtotal or Index & 198.8 & 229.8 & 229.8 & 229.8 & 37,000 \\
\hline \multicolumn{6}{|l|}{ Subsurface Maintenance, Repalr \& Services: } \\
\hline 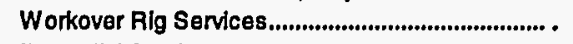 & 154.7 & 160.4 & 179.2 & 200.0 & 10,600 \\
\hline 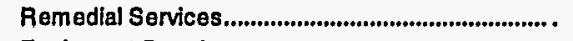 & 146.2 & 150.0 & 153.8 & 157.7 & 4,100 \\
\hline 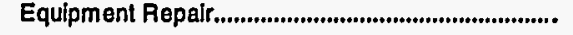 & 128.0 & 128.0 & 140.0 & 152.0 & 3,800 \\
\hline 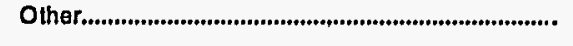 & 150.0 & 150.0 & 200.0 & 200.0 & 400 \\
\hline 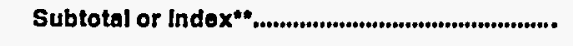 & 146.2 & 150.0 & 164.2 & 178.3 & 18,900 \\
\hline Total or Index" & 222.4 & 246.4 & 245.5 & 246.3 & 135,200 \\
\hline
\end{tabular}

Table B6. Direct Annual Operating Costs and Indices for Primary Oil Production in South Texas (10 Wells Producing from 4,000 Foot by Gas Lift)

\begin{tabular}{|c|c|c|c|c|c|}
\hline \multirow[t]{2}{*}{ Component } & \multicolumn{4}{|c|}{ Index $(1976=100)$} & \multirow{2}{*}{$\begin{array}{c}1995^{\circ} \\
\text { Cost } \\
\text { (dollars) }\end{array}$} \\
\hline & 1992 & 1993 & 1994 & 1995 & \\
\hline \multicolumn{6}{|l|}{ Normal Dally Expense: } \\
\hline 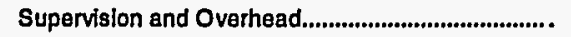 & 304.4 & 316.2 & 314.7 & 323.5 & 22,000 \\
\hline 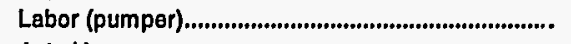 & 274.4 & 317.4 & 317.4 & 317.4 & 27,300 \\
\hline 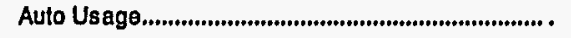 & 268.0 & 268.0 & 272.0 & 284.0 & 7,100 \\
\hline 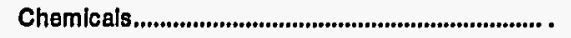 & 218.5 & 222.2 & 222.2 & 222.2 & 6,000 \\
\hline Fuel, Power \& Water................................................ & 260.7 & 305.1 & 273.5 & 178.6 & 20,900 \\
\hline 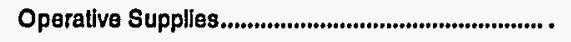 & 187.5 & 206.3 & 206.3 & 209.4 & 6,700 \\
\hline Subtotal or Index & 263.1 & 292.4 & 282.0 & 253.5 & 90,000 \\
\hline \multicolumn{6}{|l|}{ Surface Maintenance, Repalr \& Servlces: } \\
\hline Labor (roustabout) & 187.3 & 221.1 & 221.1 & 221.1 & 15,700 \\
\hline Supplies \& Senvices .................................................. . & 184.2 & 198.4 & 199.2 & 202.8 & 51,300 \\
\hline 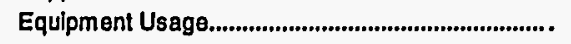 & 216.7 & 237.5 & 237.5 & 237.5 & 5,700 \\
\hline 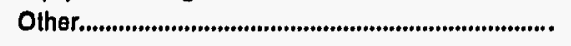 & 300.0 & 337.5 & 337.5 & 337.5 & 5,400 \\
\hline Subtotal or Index & 192.0 & 211.5 & 212.1 & 214.6 & 78,100 \\
\hline \multicolumn{6}{|l|}{ Subsurface Malntenance, Repair \& Services: } \\
\hline 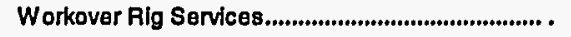 & 233.3 & 242.9 & 261.9 & 290.5 & 6,100 \\
\hline 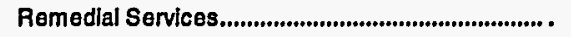 & 180.0 & 185.7 & 191.4 & 200.0 & 7,000 \\
\hline 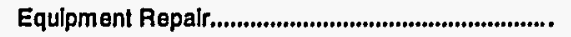 & 188.9 & 200.0 & 211.1 & 244.4 & 2,200 \\
\hline 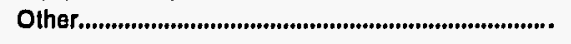 & 166.7 & 166.7 & 166.7 & 166.7 & 500 \\
\hline Subtotal or IndeX & 187.1 & 204.4 & 214.7 & 232.4 & 15,800 \\
\hline Total or Index & 224.5 & 247.4 & 243.8 & 233.7 & 183,900 \\
\hline
\end{tabular}


Table B7. Direct Annual Operating Costs and Indices for Primary Oil Production in South Texas (10 Wells Producing from 8,000 Feet by Gas Lift)

\begin{tabular}{|c|c|c|c|c|c|}
\hline \multirow[t]{2}{*}{ Component } & \multicolumn{4}{|c|}{ Index $(1976=100)$} & \multirow{2}{*}{$\begin{array}{c}1995^{*} \\
\text { Cost } \\
\text { (dollars) }\end{array}$} \\
\hline & 1992 & 1993 & 1994 & 1995 & \\
\hline \multicolumn{6}{|l|}{ Normal Daily Expense: } \\
\hline Supervision and Overhead....................................... & 310.3 & 323.1 & 321.8 & 330.8 & 25,800 \\
\hline Labor (pumper) & 274.4 & 317.4 & 317.4 & 317.4 & 27,300 \\
\hline 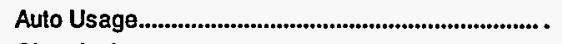 & 268.0 & 268.0 & 272.0 & 284.0 & 7,100 \\
\hline 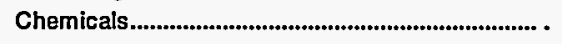 & 214.8 & 218.5 & 218.5 & 214.8 & 5,800 \\
\hline 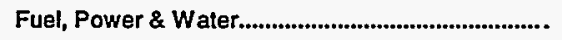 & 257.1 & 301.5 & 269.9 & 227.1 & 30,200 \\
\hline Operative Supplies.................................................... & 197.1 & 217.1 & 217.1 & 220.0 & 7,700 \\
\hline Subtotal or Index $x^{*}$ & 264.1 & 293.8 & 282.0 & 270.6 & 103,900 \\
\hline \multicolumn{6}{|l|}{ Surface Maintenance, Repair \& Services: } \\
\hline Labor (roustabout) $\ldots . . . \ldots \ldots \ldots \ldots$ & 187.3 & 221.1 & 221.1 & 221.1 & 15,700 \\
\hline 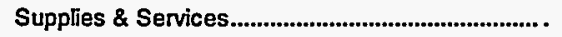 & 199.3 & 214.6 & 214.9 & 219.2 & 61,600 \\
\hline 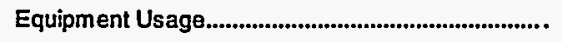 & 228.0 & 248.0 & 248.0 & 248.0 & 6,200 \\
\hline 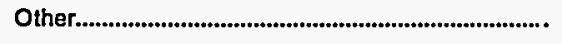 & 275.0 & 315.0 & 315.0 & $\mathbf{3 1 5 . 0}$ & 6,300 \\
\hline Subtotal or Index" & 202.8 & 222.9 & 223.2 & 226.2 & 89,800 \\
\hline \multicolumn{6}{|l|}{ Subsurface Maintenance, Repair \& Services: } \\
\hline 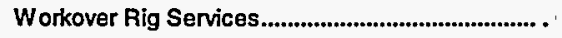 & 224.2 & 230.3 & 248.5 & 269.7 & 8,900 \\
\hline 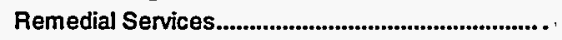 & 146.7 & 151.1 & 158.9 & 170.0 & 15,300 \\
\hline 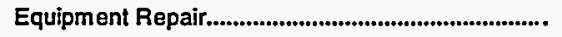 & 180.0 & 180.0 & 193.3 & 226.7 & 3,400 \\
\hline 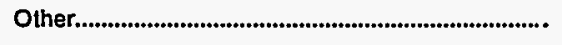 & 166.7 & 166.7 & 166.7 & 177.8 & 1,600 \\
\hline Subtotal or Index $*$ & 168.7 & 172.8 & 183.0 & 198.6 & 29,200 \\
\hline Total or Index & 222.7 & 244.3 & 241.5 & 240.2 & 222,900 \\
\hline
\end{tabular}

Table B8. Direct Annual Operating Costs and Indices for Primary Oil Production in South Texas (10 Wells Producing from 12,000 Feet by Hydraulic Lift)

\begin{tabular}{|c|c|c|c|c|c|}
\hline \multirow[t]{2}{*}{ Component } & \multicolumn{4}{|c|}{ Index $(1976=100)$} & \multirow{2}{*}{$\begin{array}{c}\text { 1995* } \\
\text { Cost } \\
\text { (doltars) }\end{array}$} \\
\hline & 1992 & 1993 & 1994 & 1995 & \\
\hline Normal Daily Expense: & & & & & \\
\hline 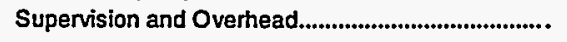 & 308.2 & 320.4 & 318.4 & 327.6 & 32,100 \\
\hline Labor (pumper) & 274.4 & 317.4 & 317.4 & 317.4 & 27,300 \\
\hline 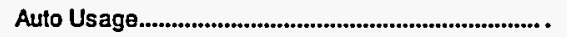 & 268.0 & 268.0 & 272.0 & 284.0 & 7,100 \\
\hline 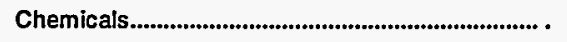 & 206.5 & 206.5 & 206.5 & 206.5 & 6,400 \\
\hline 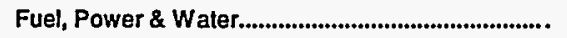 & 232.6 & 273.8 & 244.4 & 219.8 & 41,100 \\
\hline Operative Supplies .......................................................... & 278.6 & 314.3 & 314.3 & 314.3 & 4,400 \\
\hline 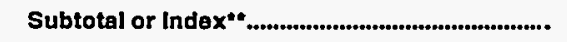 & 259.2 & 288.9 & 276.2 & 268.5 & 118,400 \\
\hline \multicolumn{6}{|l|}{ Surface Maintenance, Repair \& Services: } \\
\hline Labor (roustabout) & 187.3 & 221.1 & 221.1 & 221.1 & 15,700 \\
\hline 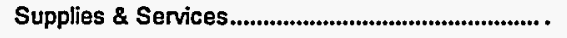 & 213.3 & 233.3 & 233.3 & 234.2 & 28,100 \\
\hline 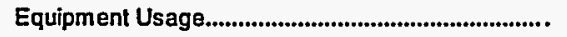 & 228.0 & 248.0 & 248.0 & 248.0 & 6,200 \\
\hline 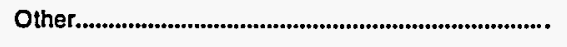 & 216.7 & 216.7 & 216.7 & 216.7 & 1,300 \\
\hline Subtotal or Index & 206.8 & 230.6 & 230.6 & 231.1 & 51,300 \\
\hline \multicolumn{6}{|l|}{ Subsurface Maintenance, Repair \& Services: } \\
\hline Workover Rig Services ........................................... . & 195.7 & 198.6 & 210.0 & 221.4 & 15,500 \\
\hline 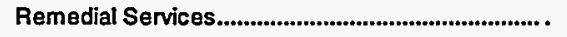 & 220.4 & 225.7 & 239.8 & 256.1 & 68,900 \\
\hline 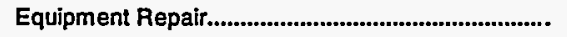 & 249.2 & 250.3 & 403.4 & 421.2 & 75,400 \\
\hline 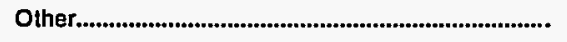 & 153.8 & 153.8 & 161.5 & 161.5 & 2,100 \\
\hline 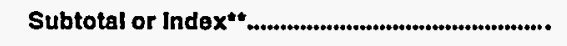 & 225.2 & 228.6 & 289.1 & 304.9 & 161,900 \\
\hline Total or index**.. & 234.3 & 251.3 & 273.5 & 277.7 & 331,600 \\
\hline
\end{tabular}


Table C1. Lease Equipment Costs and Indices for Primary Oil Production in South Loulsiana (10 Wells Producing from 2,000 Feet by Rod Lift)

\begin{tabular}{|c|c|c|c|c|c|}
\hline \multirow{2}{*}{ Component } & \multicolumn{4}{|c|}{ Index $(1976=100)$} & \multirow{2}{*}{$\begin{array}{c}\text { 1995* } \\
\text { Cost } \\
\text { (dollars) }\end{array}$} \\
\hline & 1992 & 1993 & 1994 & 1995 & \\
\hline \multicolumn{6}{|l|}{ Producing Equipment: } \\
\hline 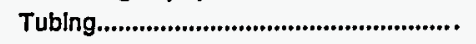 & 108.5 & 109.6 & 110.2 & 155.0 & 54,700 \\
\hline 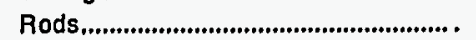 & 107.8 & 107.4 & 123.0 & 123.0 & 25,100 \\
\hline 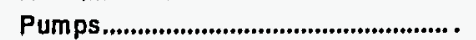 & 131.0 & 133.3 & 147.6 & 152.4 & 12,800 \\
\hline Pumping Equipment.................................. & 164.7 & 174.3 & 179.1 & 191.4 & 246,000 \\
\hline 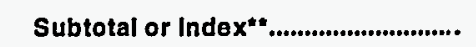 & 146.9 & 153.6 & 159.1 & 175.8 & 338,600 \\
\hline \multicolumn{6}{|l|}{ Gathering System: } \\
\hline 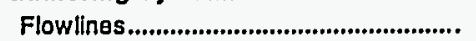 & 244.2 & 243.9 & 263.0 & 269.1 & 88,800 \\
\hline 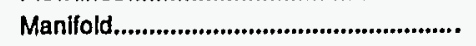 & 262.4 & 261.7 & 264.7 & 267.7 & 35,600 \\
\hline 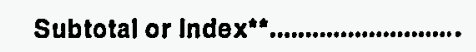 & 249.5 & 249.0 & 263.5 & 268.7 & 124,400 \\
\hline \multicolumn{6}{|l|}{ Lease Equipment: } \\
\hline Producing Separator................................ . & 165.6 & 168.8 & 173.4 & 173.4 & 11,100 \\
\hline 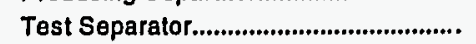 & 190.1 & 193.1 & 189.1 & 188.1 & 19,000 \\
\hline 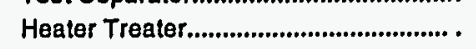 & 152.3 & 153.5 & 136.8 & 145.8 & 22,600 \\
\hline 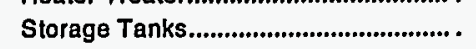 & 187.8 & 191.3 & 196.7 & 203.3 & 68,100 \\
\hline Accessory Equlpment................................. & 206.8 & 210.9 & 212.9 & 220.4 & 32,400 \\
\hline 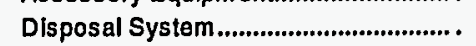 & 178.2 & 181.3 & 184.9 & 189.7 & 79,300 \\
\hline 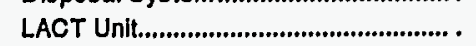 & 169.9 & 174.2 & 174.2 & 171.0 & 15,900 \\
\hline 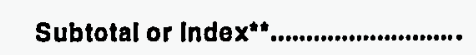 & 180.5 & 183.7 & 184.4 & 189.2 & 248,400 \\
\hline Total or Index & 266.0 & 273.0 & 280.7 & 297.8 & 711,400 \\
\hline
\end{tabular}

Table C2. Lease Equipment Costs and Indices for Primary Oil Production in South Louisiana (10 Wells Producing from 4,000 Feet by Gas Lift)

\begin{tabular}{|c|c|c|c|c|c|}
\hline \multirow{2}{*}{ Component } & \multicolumn{4}{|c|}{ Index $(1976=100)$} & \multirow{2}{*}{$\begin{array}{c}\text { 1995* } \\
\text { Cost } \\
\text { (dollars) }\end{array}$} \\
\hline & 1992 & 1993 & 1994 & 1995 & \\
\hline \multicolumn{6}{|l|}{ Producing Equipment: } \\
\hline 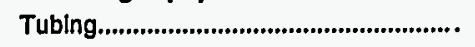 & 105.6 & 106.8 & 107.4 & 151.5 & 108,600 \\
\hline 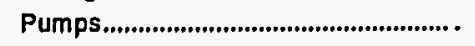 & 342.7 & 349.8 & 390.1 & 394.5 & 99,800 \\
\hline Pumping Equipment............................... & 160.1 & 162.9 & 163.9 & 169.9 & 177,400 \\
\hline Subtotal or Index *............................. & 163.6 & 166.4 & 172.2 & 191.6 & 385,800 \\
\hline \multicolumn{6}{|l|}{ Gathering System: } \\
\hline 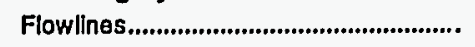 & 224.7 & 224.1 & 242.8 & 251.0 & 197,500 \\
\hline 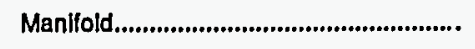 & 262.4 & 261.7 & 264.7 & 267.7 & 35,600 \\
\hline Subtotal or Index $x^{* \star \ldots . . . . . . . . . . . . . . . . . . . . . . . . . . . . ~}$ & 230.1 & 229.6 & 246.0 & 253.4 & 233,100 \\
\hline \multicolumn{6}{|l|}{ Lease Equipment: } \\
\hline Produclng Separator.............................. . & 165.6 & 168.8 & 173.4 & 173.4 & 11,100 \\
\hline Test Separator..................................... & 190.1 & 193.1 & 189.1 & 188.1 & 19,000 \\
\hline Heater Treater........................................ . & 152.3 & 153.5 & 136.8 & 145.8 & 22,600 \\
\hline Storage Tanks...................................... & 187.8 & 191.3 & 196.7 & 203.3 & 68,100 \\
\hline Accessory Equlpment.......................... & 206.8 & 210.9 & 212.9 & 220.4 & 32,400 \\
\hline Disposal System.................................. & 178.6 & 181.6 & 185.6 & 190.2 & 81,800 \\
\hline 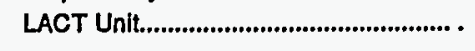 & 169.9 & 174.2 & 174.2 & 171.0 & 15,900 \\
\hline Subtotal or Index & 180.6 & 183.8 & 184.6 & 189.4 & 250,900 \\
\hline Total or Index & 183.3 & 185.5 & 192.0 & 204.2 & 869,800 \\
\hline
\end{tabular}


Table C3. Lease Equipment Costs and Indices for Primary Oil Production in South Louisiana (10 Wells Producing from 8,000 Feet by Gas Lift)

\begin{tabular}{|c|c|c|c|c|c|}
\hline \multirow{2}{*}{ Component } & \multicolumn{4}{|c|}{ Index $(1976=100)$} & \multirow{2}{*}{$\begin{array}{c}\text { 1995* } \\
\text { Cost } \\
\text { (dollars) }\end{array}$} \\
\hline & 1992 & 1993 & 1994 & 1995 & \\
\hline \multicolumn{6}{|l|}{ Producing Equipment: } \\
\hline 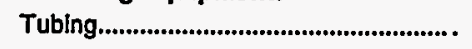 & 105.7 & 107.0 & 107.5 & 151.7 & 217,200 \\
\hline 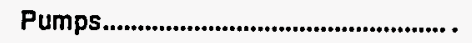 & 342.9 & 350.0 & 390.4 & 394.9 & 139,800 \\
\hline Pumping Equipment.................................. & 158.4 & 161.2 & 162.2 & 168.2 & 177,400 \\
\hline Subtotal or Index" "................................ & 154.8 & 157.4 & 163.1 & 188.1 & 534,400 \\
\hline \multicolumn{6}{|l|}{ Gathering System: } \\
\hline 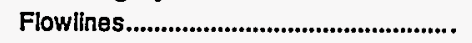 & 220.6 & 220.0 & 238.8 & 247.4 & 305,600 \\
\hline 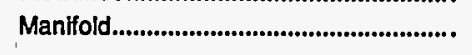 & 262.4 & 261.7 & 264.7 & 267.7 & 35,600 \\
\hline 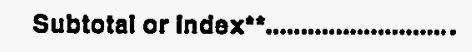 & 224.7 & 224.0 & 241.3 & 249.4 & 341,200 \\
\hline \multicolumn{6}{|l|}{ Lease Equipment: } \\
\hline 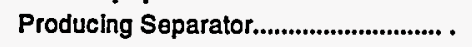 & 165.6 & 168.8 & 173.4 & 173.4 & 11,100 \\
\hline 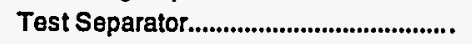 & 190.1 & 193.1 & 189.1 & 188.1 & 19,000 \\
\hline 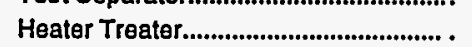 & 152.3 & 153.5 & 136.8 & 145.8 & 22,600 \\
\hline 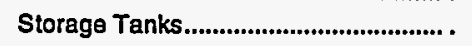 & 187.8 & 191.3 & 196.7 & 203.3 & 68,100 \\
\hline 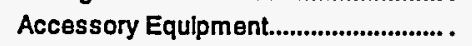 & 206.8 & 210.9 & 212.9 & 220.4 & 32,400 \\
\hline 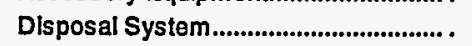 & 183.5 & 185.5 & 191.4 & 197.8 & 80,300 \\
\hline 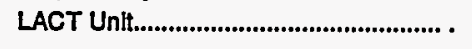 & 169.9 & 174.2 & 174.2 & 171.0 & 15,900 \\
\hline Subtotal or Index**.................................. & 182.2 & 185.0 & 186.4 & 191.7 & 249,400 \\
\hline 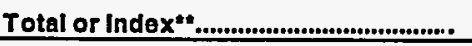 & 178.6 & 180.5 & 188.0 & 204.2 & $1,125,000$ \\
\hline
\end{tabular}

Table C4. Lease Equipment Costs and Indices for Primary Oll Production in South Louisiana (10 Wells Producing from 12,000 Feet by Hydraulic Lift)

\begin{tabular}{|c|c|c|c|c|c|}
\hline \multirow{2}{*}{ Component } & \multicolumn{4}{|c|}{ Index $(1976=100)$} & \multirow{2}{*}{$\begin{array}{c}\text { 1995* } \\
\text { Cost } \\
\text { (dollars) }\end{array}$} \\
\hline & 1992 & 1993 & 1994 & 1995 & \\
\hline \multicolumn{6}{|l|}{ Producing Equipment: } \\
\hline 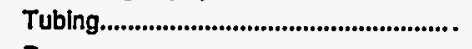 & 208.1 & 163.7 & 137.3 & 144.9 & 731,400 \\
\hline 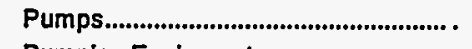 & 153.4 & 153.4 & 250.5 & 261.7 & 196,000 \\
\hline Pumping Equipment.................................... & 191.2 & 191.0 & 173.8 & 179.6 & 319,300 \\
\hline Subtotal or Index $=$ & 198.7 & 169.1 & 157.1 & 164.6 & $1,246,700$ \\
\hline \multicolumn{6}{|l|}{ Gathering System: } \\
\hline 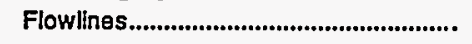 & 234.3 & 213.7 & 207.8 & 215.3 & 285,000 \\
\hline 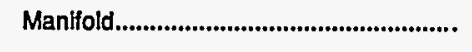 & 262.4 & 261.7 & 264.7 & 267.7 & 35,600 \\
\hline 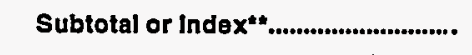 & 236.9 & 218.1 & 213.0 & 220.0 & 320,600 \\
\hline \multicolumn{6}{|l|}{ Lease Equipment: } \\
\hline Producing Separator................................. . & 165.6 & 168.8 & 173.4 & 173.4 & 11,100 \\
\hline 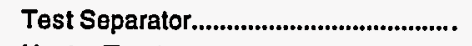 & 190.1 & 193.1 & 189.1 & 188.1 & 19,000 \\
\hline Heater Treater....................................... . & 152.3 & 153.5 & 136.8 & 145.8 & 22,600 \\
\hline 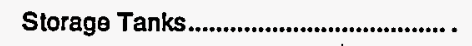 & 187.8 & 191.3 & 196.7 & 203.3 & 68,100 \\
\hline Accessory Equipment................................ & 206.8 & 210.9 & 212.9 & 220.4 & 32,400 \\
\hline 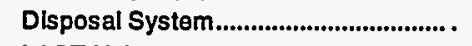 & 196.5 & 198.7 & 204.5 & 210.3 & 83,700 \\
\hline 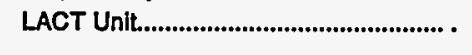 & 169.9 & 174.2 & 174.2 & 171.0 & 15,900 \\
\hline 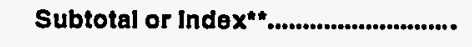 & 186.2 & 189.1 & 190.4 & 195.5 & 252,800 \\
\hline Total or Index ${ }^{* *} \ldots$ & 202.5 & 178.5 & 169.1 & 176.3 & $1,820,100$ \\
\hline
\end{tabular}


Table C5. Direct Annual Operating Costs and Indlces for Primary Oil Production in South Loulslana (10 Wells Producing from 2,000 Feet by Rod Lift)

\begin{tabular}{|c|c|c|c|c|c|}
\hline \multirow[t]{2}{*}{ Component } & \multicolumn{4}{|c|}{ Index $(1976=100)$} & \multirow{2}{*}{$\begin{array}{c}\text { 1995* } \\
\text { Coat } \\
\text { (dollara) }\end{array}$} \\
\hline & 1992 & 1993 & 1994 & 1995 & \\
\hline \multicolumn{6}{|l|}{ Normal Daily Expense: } \\
\hline 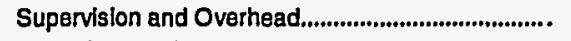 & 290.0 & 293.3 & 300.0 & 303.3 & 18,200 \\
\hline 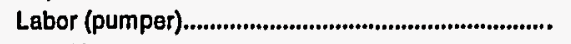 & 232.0 & 240.7 & 257.0 & 261.0 & 44,900 \\
\hline 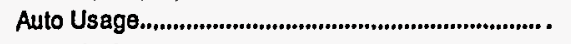 & 268.0 & 268.0 & 272.0 & 284.0 & 7,100 \\
\hline 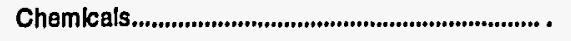 & 200.0 & 203.7 & 203.7 & 203.7 & 5,500 \\
\hline 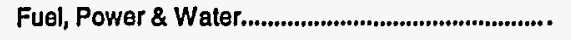 & 326.0 & 386.0 & 376.0 & 364.0 & 18,200 \\
\hline Operative Supplles........................................................... & 214.3 & 214.3 & 214.3 & 214.3 & 1,500 \\
\hline Subtotal or Index" & 255.7 & 269.8 & 278.0 & 279.8 & 95,400 \\
\hline \multicolumn{6}{|l|}{ Surface Maintenance, Repair \& Servlces: } \\
\hline 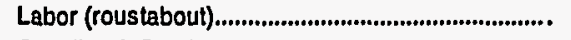 & 192.1 & 200.0 & 205.3 & 210.5 & 8,000 \\
\hline 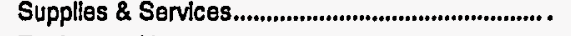 & 202.8 & 197.2 & 191.7 & 183.3 & 6,600 \\
\hline 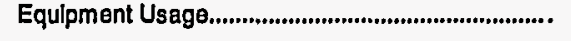 & 356.3 & 356.3 & 375.0 & 393.8 & 6,300 \\
\hline Subtotal or Index" & 225.6 & 226.7 & 230.0 & 232.2 & 20,900 \\
\hline \multicolumn{6}{|l|}{ Subsurface Maintenance, Repair \& Services: } \\
\hline 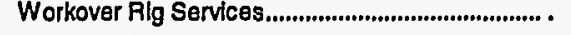 & 198.3 & 196.7 & 206.7 & 206.7 & 12,400 \\
\hline 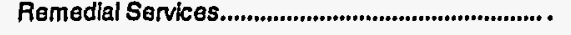 & 138.5 & 138.5 & 142.3 & 146.2 & 3,800 \\
\hline 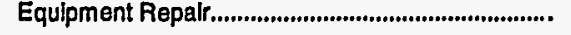 & 132.0 & 132.0 & 144.0 & 152.0 & 3,800 \\
\hline 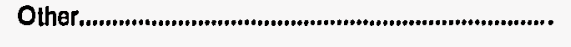 & 150.0 & 150.0 & 200.0 & 200.0 & 400 \\
\hline 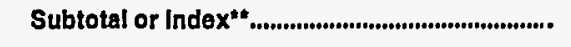 & 169.0 & 168.1 & 177.9 & 180.5 & 20,400 \\
\hline 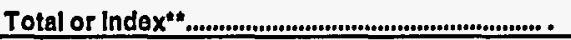 & 232.7 & 241.5 & 249.3 & 251.3 & 136,700 \\
\hline
\end{tabular}

Table C6. DIrect Annual Operating Cost8 and Indices for Primary Oll Production in South Loulsiana (10 Wells Producing from 4,000 Feet by Gas Lift)

\begin{tabular}{|c|c|c|c|c|c|c|}
\hline \multirow[t]{2}{*}{ Component } & \multicolumn{5}{|c|}{ Index $(1976=100)$} & \multirow{2}{*}{$\begin{array}{c}\text { 1995* } \\
\text { Cost } \\
\text { (dollara) }\end{array}$} \\
\hline & 1992 & & 1993 & 1994 & 1995 & \\
\hline \multicolumn{7}{|l|}{ Normal Dally Expense: } \\
\hline Supervislon and Overhead....................................... & 289.7 & & 294.1 & 300.0 & 302.9 & 20,600 \\
\hline Labor (pumper) & 232.0 & & 240.7 & 257.0 & 261.0 & 44,900 \\
\hline 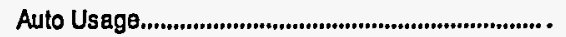 & 268.0 & & 268.0 & 272.0 & 284.0 & 7,100 \\
\hline 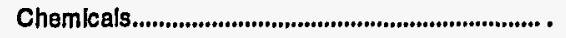 & 211.1 & & 211.1 & 214.8 & 214.8 & 5,800 \\
\hline 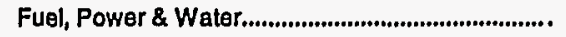 & 362.0 & & 436.7 & 424.1 & 410.1 & 32,400 \\
\hline Operative Supplies......................................................... & 186.2 & & 193.1 & 196.6 & 200.0 & 5,800 \\
\hline Subtotal or Index ${ }^{\star \star *} \ldots \ldots \ldots \ldots \ldots \ldots$ & 265.0 & & 284.8 & 291.0 & 291.5 & 116,600 \\
\hline \multicolumn{7}{|l|}{ Surface Malntenance, Repair \& Services: } \\
\hline Labor (roustabout) & 192.1 & $"$ & 200.0 & 205.3 & 210.5 & 8,000 \\
\hline Supplles \& Services,................................................ & 186.6 & & 189.3 & 193.7 & 199.2 & 50,400 \\
\hline 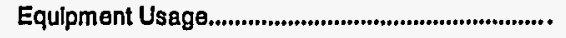 & 347.1 & & 347.1 & 364.7 & 382.4 & 6,500 \\
\hline Subtotal or Index & 196.1 & & 199.4 & 204.5 & 210.7 & 64,900 \\
\hline \multicolumn{7}{|l|}{ Subsurface Maintenance, Repalr \& Services: } \\
\hline Workover Rlg Services.......................................... . & 196.3 & & 196.3 & 207.4 & 203.7 & 5,500 \\
\hline 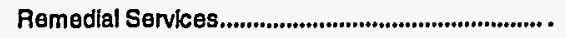 & 151.4 & & 154.3 & 160.0 & 162.9 & 5,700 \\
\hline Equlpment Repalt & 188.9 & & 200.0 & 211.1 & 244.4 & 2,200 \\
\hline Other,..., & 166.7 & & 166.7 & 166.7 & 166.7 & 500 \\
\hline Subtotal or Index & 173.0 & & 175.7 & 183.8 & 187.8 & 13,900 \\
\hline Total or Index & 229.2 & & 240.8 & 246.8 & 249.9 & 195,400 \\
\hline
\end{tabular}


Table C7. Direct Annual Operating Costs and Indices for Primary Oil Production in South Louisiana (10 Wells Producing from 8,000 Feet by Gas Lift)

\begin{tabular}{|c|c|c|c|c|c|}
\hline \multirow[t]{2}{*}{ Component } & \multicolumn{4}{|c|}{ Index $(1976=100)$} & \multirow{2}{*}{$\begin{array}{c}1995^{4} \\
\text { Cost } \\
\text { (dollars) }\end{array}$} \\
\hline & 1992 & 1993 & 1994 & 1995 & \\
\hline \multicolumn{6}{|l|}{ Normal Daily Expense: } \\
\hline 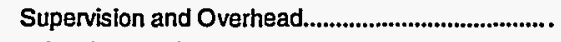 & 293.7 & 298.7 & 303.8 & 307.6 & 24,300 \\
\hline Labor (pumper) & 232.0 & 240.7 & 257.0 & 261.0 & 44,900 \\
\hline Auto Usage & 268.0 & 268.0 & 272.0 & 284.0 & 7,100 \\
\hline 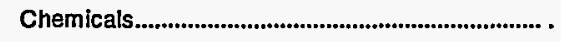 & 254.5 & 254.5 & 259.1 & 259.1 & 5,700 \\
\hline 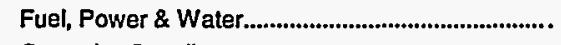 & 358.9 & 434.4 & 422.2 & 407.8 & 36,700 \\
\hline 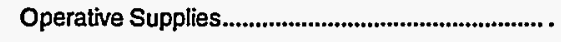 & 203.1 & 206.3 & 212.5 & 218.8 & 7,000 \\
\hline Subtotal or Index & 271.9 & 292.9 & 298.8 & 299.3 & 125,700 \\
\hline \multicolumn{6}{|l|}{ Surface Maintenance, Repair \& Services: } \\
\hline 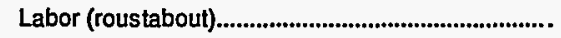 & 192.1 & 200.0 & 205.3 & 210.5 & 8,000 \\
\hline 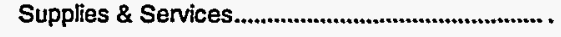 & 206.0 & 209.3 & 213.9 & 220.3 & 61,900 \\
\hline 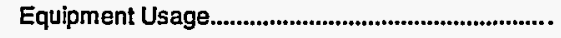 & 355.6 & 361.1 & 383.3 & 405.6 & 7,300 \\
\hline Subtotal or Index ${ }^{* *} \ldots$ & 212.5 & 216.3 & 222.0 & 229.1 & 77,200 \\
\hline \multicolumn{6}{|l|}{ Subsurface Maintenance, Repair \& Services: } \\
\hline 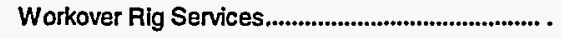 & 274.4 & 274.4 & 287.2 & 287.2 & 11,200 \\
\hline 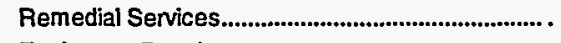 & 152.2 & 153.3 & 158.9 & 160.0 & 14,400 \\
\hline 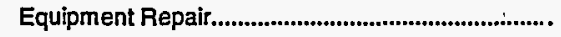 & 192.9 & 192.9 & 207.1 & 242.9 & 3,400 \\
\hline 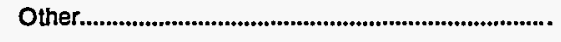 & 166.7 & 166.7 & 166.7 & 177.8 & 1,600 \\
\hline 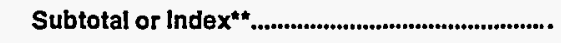 & 188.2 & 188.8 & 196.7 & 201.3 & 30,600 \\
\hline Total or Index & 235.9 & 247.1 & 253.2 & 256.9 & 233,500 \\
\hline
\end{tabular}

Table C8. Direct Annual Operating Costs and Indices for Primary OIl Production in South Louisiana (10 Wells Producing from 12,000 Feet by Hydraulic Lift)

\begin{tabular}{|c|c|c|c|c|c|}
\hline \multirow[t]{2}{*}{ Component } & \multicolumn{4}{|c|}{ Index $(1976=100)$} & \multirow{2}{*}{$\begin{array}{c}1995^{\circ} \\
\text { Cost } \\
\text { (dollars) }\end{array}$} \\
\hline & 1992 & 1993 & 1994 & 1995 & \\
\hline \multicolumn{6}{|l|}{ Normal Daily Expense: } \\
\hline 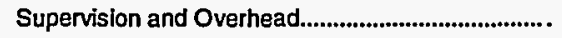 & 295.9 & 301.0 & 307.1 & 309.2 & 30,300 \\
\hline Labor (pumper) & 232.0 & 240.7 & 257.0 & 261.0 & 44,900 \\
\hline 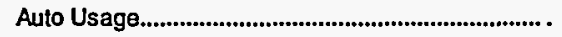 & 268.0 & 268.0 & 272.0 & 284.0 & 7,100 \\
\hline 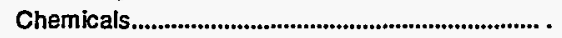 & 192.9 & 196.4 & 196.4 & 196.4 & 5,500 \\
\hline 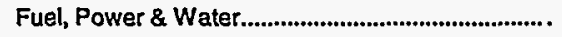 & 350.8 & 426.3 & 415.3 & 400.0 & 47,200 \\
\hline 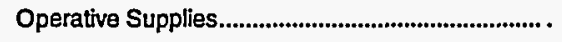 & 214.3 & 221.4 & 228.6 & 228.6 & 3,200 \\
\hline 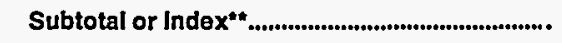 & 275.6 & 300.0 & 305.1 & 303.7 & 138,200 \\
\hline \multicolumn{6}{|l|}{ Surface Maintenance, Repair \& Services: } \\
\hline 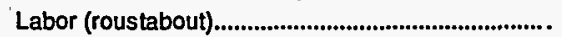 & 192.1 & 200.0 & 205.3 & 210.5 & 8,000 \\
\hline 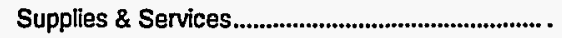 & 212.3 & 223.6 & 225.5 & 226.4 & 24,000 \\
\hline 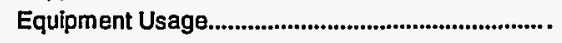 & 355.6 & 361.1 & 383.3 & 405.6 & 7,300 \\
\hline 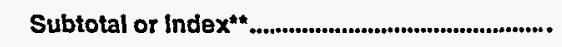 & 223.5 & 233.3 & 238.3 & 242.6 & 39,300 \\
\hline \multicolumn{6}{|l|}{ Subsurface Maintenance, Repair \& Services: } \\
\hline Workover Rig Services................................................. . & 188.2 & 188.2 & 193.4 & 193.4 & 14,700 \\
\hline Remedial Services................................................... & 175.1 & 175.5 & 183.3 & $184^{\circ} 4^{\circ}$ & 49,600 \\
\hline 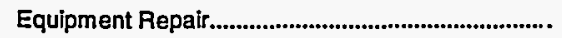 & 260.9 & 262.1 & 423.0 & 441.4 & 76,800 \\
\hline 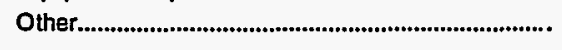 & 166.7 & 166.7 & 175.0 & 175.0 & 2,100 \\
\hline 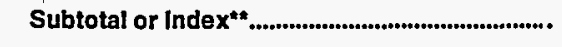 & 204.9 & 205.5 & 263.1 & 269.7 & 143,200 \\
\hline Total or Index ${ }^{* *}$ & 235.5 & 246.9 & 276.2 & 279.4 & 320,700 \\
\hline
\end{tabular}


Table D1. Lease Equipment Costs and Indices for Primary Oll Production in Oklahoma (10 Wells Producing from 2,000 Feet by Rod Litt)

\begin{tabular}{|c|c|c|c|c|c|}
\hline \multirow{2}{*}{ Component } & \multicolumn{4}{|c|}{ Index $(1976=100)$} & \multirow{2}{*}{$\begin{array}{c}\text { 1995* } \\
\text { Cost } \\
\text { (dollars) }\end{array}$} \\
\hline & 1992 & 1993 & 1994 & 1995 & \\
\hline \multicolumn{6}{|l|}{ Producing Equipment: } \\
\hline 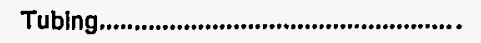 & 107.6 & 108.8 & 109.3 & 154.0 & 54,500 \\
\hline 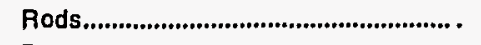 & 100.5 & 100.5 & 112.3 & 112.3 & 22,900 \\
\hline 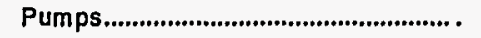 & 131.0 & 133.3 & 147.6 & 152.4 & 12,800 \\
\hline Pumping Equipment.............................. & 161.5 & 171.4 & 176.1 & 188.2 & 245,400 \\
\hline Subtotal or Index & 144.0 & 150.9 & 156.0 & 172.5 & 335,600 \\
\hline \multicolumn{6}{|l|}{ Gathering System: } \\
\hline 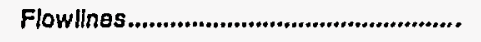 & 253.2 & 255.6 & 261.1 & 266.7 & 33,600 \\
\hline Manifold.................................................. & 262.9 & 261.4 & 264.4 & 268.2 & 35,400 \\
\hline 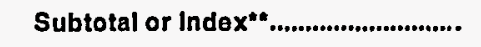 & 258.1 & 258.5 & 262.8 & 267.4 & 69,000 \\
\hline \multicolumn{6}{|l|}{ Lease Equipment: } \\
\hline Producing Separator............................ . & 165.6 & 168.8 & 173.4 & 173.4 & 11,100 \\
\hline Test Separator....................................... & 190.1 & 193.1 & 189.1 & 188.1 & 19,000 \\
\hline Heater Treater........................................ . & 152.3 & 153.5 & 136.8 & 145.8 & 22,600 \\
\hline 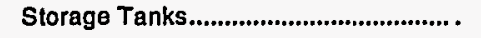 & 189.0 & 192.2 & 195.2 & 200.9 & 67,300 \\
\hline Accessory Equipm ent............................ . & 206.8 & 210.9 & 212.9 & 220.4 & 32,400 \\
\hline Dlsposal System................................... & 178.2 & 184.2 & 183.7 & 187.5 & 74,800 \\
\hline LACT Unit............................................ . & 169.9 & 174.2 & 174.2 & 171.0 & 15,900 \\
\hline Subtotal or Index & 180.8 & 184.9 & 183.6 & 187.9 & 243,100 \\
\hline Total or Index & 263.5 & 272.1 & 276.3 & 293.9 & 647,700 \\
\hline
\end{tabular}

Table D2. Lease Equipment Costs and Indices for Primary Oil Production in Oklahoma (10 Wells Producing from 4,000 Feet by Rod Litt)

\begin{tabular}{|c|c|c|c|c|c|}
\hline \multirow{2}{*}{ Component } & \multicolumn{4}{|c|}{ Index $(1976=100)$} & \multirow{2}{*}{$\begin{array}{c}\text { 1995* } \\
\text { Cost } \\
\text { (dollars) }\end{array}$} \\
\hline & 1992 & 1993 & 1994 & 1995 & \\
\hline \multicolumn{6}{|l|}{ Producing Equipment: } \\
\hline 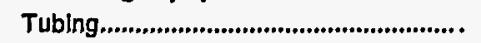 & 105.3 & 106.4 & 107.0 & 151.1 & 108,500 \\
\hline 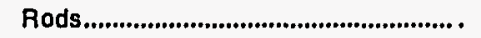 & 97.5 & 97.8 & 108.1 & 108.1 & 43,800 \\
\hline Pumps................................................ & 132.9 & 136.6 & 134.1 & 154.9 & 12,700 \\
\hline Pumping Equipment............................. & 146.2 & 156.0 & 158.7 & 178.0 & 397,200 \\
\hline Subtotal or Index & 131.6 & 138.3 & 141.4 & 163.6 & 562,200 \\
\hline \multicolumn{6}{|l|}{ Gathering System: } \\
\hline 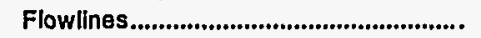 & 238.7 & 241.1 & 246.0 & 251.5 & 41,000 \\
\hline Manifold.................................................. & 262.9 & 261.4 & 264.4 & 268.2 & 35,400 \\
\hline Subtotal or Index*"............................ & 249.5 & 250.2 & 254.2 & 259.0 & 76,400 \\
\hline \multicolumn{6}{|l|}{ Lease Equipment: } \\
\hline Producing Separator............................ . & 165.6 & 168.8 & 173.4 & 173.4 & 11,100 \\
\hline Test Separator..................................... & 190.1 & 193.1 & 189.1 & 188.1 & 19,000 \\
\hline Heater Treater......................................... . & 152.3 & 153.5 & 136.8 & 145.8 & 22,600 \\
\hline Storage Tanks....................................... & 189.0 & 192.2 & 195.2 & 200.9 & 67,300 \\
\hline Accessory Equlpment....................... & 206.8 & 210.9 & 212.9 & 220.4 & 32,400 \\
\hline Disposal System................................. & 208.6 & 215.6 & 214.9 & 219.3 & 89,700 \\
\hline LACT Unit........................................... & 169.9 & 174.2 & 174.2 & 171.0 & 15,900 \\
\hline Subtotal or Index ${ }^{\star *} \ldots \ldots . . . . . . . . . . . . . . . . . . . .$. & 190.3 & 194.7 & 193.4 & 197.9 & 258,000 \\
\hline Total or Index*" & 207.4 & 215.2 & 217.9 & 240.3 & 896,600 \\
\hline
\end{tabular}

Energy Information Administration

Oll \& Gas Field Equipment \& Production Operations, 1992 through 1995 
Table D3. Lease Equipment Costs and Indices for Primary Oil Production in Oklahoma

(10 Wells Producing from 8,000 Feet by Hydraulic Lift)

\begin{tabular}{|c|c|c|c|c|c|}
\hline \multirow{2}{*}{ Component } & \multicolumn{4}{|c|}{ Index $(1976=100)$} & \multirow{2}{*}{$\begin{array}{c}\text { 1995* } \\
\text { Cost } \\
\text { (dollars) }\end{array}$} \\
\hline & 1992 & 1993 & 1994 & 1995 & \\
\hline \multicolumn{6}{|l|}{ Producing Equipment: } \\
\hline 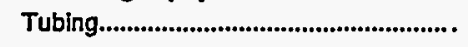 & 202.9 & 131.8 & 133.2 & 163.8 & 466,600 \\
\hline 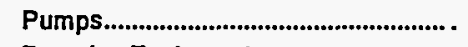 & 153.4 & 153.4 & 250.5 & 261.7 & 196,000 \\
\hline Pumping Equipment................................... & 205.2 & 205.2 & 183.9 & 189.4 & 289,400 \\
\hline Subtotal or Index $=\ldots$ & 196.4 & 156.8 & 165.5 & 185.7 & 952,000 \\
\hline \multicolumn{6}{|l|}{ Gathering System: } \\
\hline 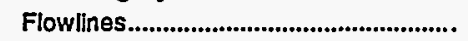 & 259.4 & 221.1 & 193.1 & 197.0 & 146,000 \\
\hline 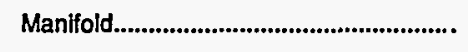 & 262.9 & 261.4 & 264.4 & 268.2 & 35,400 \\
\hline 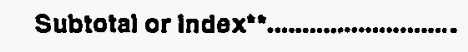 & 259.9 & 227.1 & 203.9 & 207.8 & 181,400 \\
\hline \multicolumn{6}{|l|}{ Lease Equipment: } \\
\hline Producing Separator............................... . & 165.6 & 168.8 & 173.4 & 173.4 & 11,100 \\
\hline 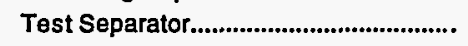 & 190.1 & 193.1 & 189.1 & 188.1 & 19,000 \\
\hline 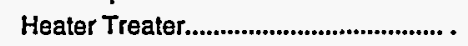 & 152.3 & 153.5 & 136.8 & 145.8 & 22,600 \\
\hline 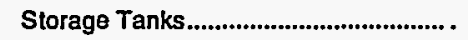 & 189.0 & 192.2 & 195.2 & 200.9 & 67,300 \\
\hline 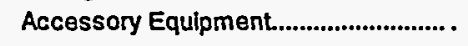 & 206.8 & 210.9 & 212.9 & 220.4 & 32,400 \\
\hline Dlsposal System & 206.2 & 214.1 & 213.1 & 217.1 & 94,200 \\
\hline 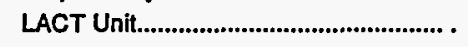 & 169.9 & 174.2 & 174.2 & 171.0 & 15,900 \\
\hline Subtotal or Index $* \pm \ldots \ldots \ldots$ & 189.9 & 194.6 & 193.2 & 197.5 & 262,500 \\
\hline Total or Index ${ }^{* *}$ & 202.8 & 172.0 & 175.1 & 190.5 & $1,395,900$ \\
\hline
\end{tabular}

Table D4. Lease Equipment Costs and Indices for Primary Oil Production in Oklahoma (10 Wells Producing from 12,000 Feet by Hydraulic Lift)

\begin{tabular}{|c|c|c|c|c|c|}
\hline \multirow{2}{*}{ Component } & \multicolumn{4}{|c|}{ Index $(1976=100)$} & \multirow{2}{*}{$\begin{array}{c}\text { 1995* } \\
\text { Cost } \\
\text { (dollars) }\end{array}$} \\
\hline & 1992 & 1993 & 1994 & 1995 & \\
\hline \multicolumn{6}{|l|}{ Producing Equlpment: } \\
\hline Tubing......................................................... & 207.3 & 163.1 & 136.7 & 144.3 & 730,200 \\
\hline 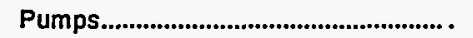 & 153.4 & 153.4 & 250.5 & 261.7 & 196,000 \\
\hline Pumping Equipment................................. & 187.6 & 187.9 & 169.7 & 174.6 & 310,500 \\
\hline Subtotal or Index & 197.4 & 167.9 & 155.7 & 163.0 & $1,236,700$ \\
\hline \multicolumn{6}{|l|}{ Gathering System: } \\
\hline 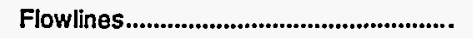 & 259.4 & 221.1 & 193.1 & 197.0 & 146,000 \\
\hline Manifold...................................................... & 262.9 & 261.4 & 264.4 & 268.2 & 35,400 \\
\hline 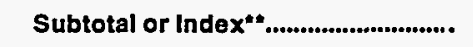 & 259.9 & 227.1 & 203.9 & 207.8 & 181,400 \\
\hline \multicolumn{6}{|l|}{ Lease Equipment: } \\
\hline Producing Separator.............................. . & 165.6 & 168.8 & 173.4 & 173.4 & 11,100 \\
\hline Test Separator......................................... & 190.1 & 193.1 & 189.1 & 188.1 & 19,000 \\
\hline Heater Treater.......................................... . & 152.3 & 153.5 & 136.8 & 145.8 & 22,600 \\
\hline 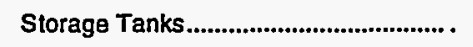 & 189.0 & 192.2 & 195.2 & 200.9 & 67,300 \\
\hline Accessory Equipment.......................... & 206.8 & 210.9 & 212.9 & 220.4 & 32,400 \\
\hline Disposal System.................................... & 180.6 & 187.5 & 186.7 & 190.6 & 68,600 \\
\hline 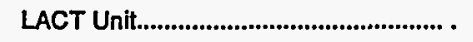 & 169.9 & 174.2 & 174.2 & 171.0 & 15,900 \\
\hline Subtotal or Index & 181.6 & 185.8 & 184.5 & 188.8 & 236,900 \\
\hline Total or Index & 201.0 & 175.6 & 163.7 & 170.4 & $1,655,000$ \\
\hline
\end{tabular}


Table D5. Direct Annual Operating Costs and Indices for Primary Oll Production in Oklahoma (10 Wells Producing from 2,000 Feet by Rod Liff)

\begin{tabular}{|c|c|c|c|c|c|}
\hline \multirow[t]{2}{*}{ Component } & \multicolumn{4}{|c|}{ Index $(1976=100)$} & \multirow{2}{*}{$\begin{array}{c}\text { 1995* } \\
\text { Cost } \\
\text { (dollars) }\end{array}$} \\
\hline & 1992 & 1993 & 1994 & 1995 & \\
\hline \multicolumn{6}{|l|}{ Normal Dally Expense: } \\
\hline 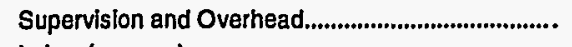 & 288.3 & 300.0 & 298.3 & 305.0 & 18,300 \\
\hline Labor (pumper) & 210.8 & 223.0 & 223.0 & 223.0 & 16,500 \\
\hline Auto Usage. & 268.0 & 268.0 & 272.0 & 284.0 & 7,100 \\
\hline 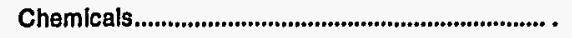 & 203.7 & 207.4 & 207.4 & 203.7 & 5,500 \\
\hline 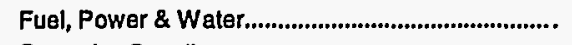 & 279.6 & 307.4 & 279.6 & 238.9 & 12,900 \\
\hline Operative Supplles....................................................... & 216.7 & 216.7 & 216.7 & 216.7 & 1,300 \\
\hline Subtotal or Index & 250.0 & 263.0 & 256.9 & 250.4 & 61,600 \\
\hline \multicolumn{6}{|l|}{ Surface Maintenance, Repair \& Services: } \\
\hline 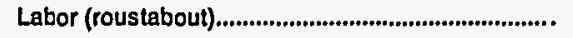 & 206.9 & 206.9 & 206.9 & 220.7 & 6,400 \\
\hline Supplles \& Services.................................................... & 218.8 & 212.5 & 206.3 & 200.0 & 6,400 \\
\hline Equipment Usage................................................... & 276.9 & 284.6 & 284.6 & 292.3 & 3,800 \\
\hline Subtotal or Index" & 224.3 & 223.0 & 220.3 & 224.3 & 16,600 \\
\hline \multicolumn{6}{|l|}{ Subsurface Maintenance, Repair \& Services: } \\
\hline Workover Rlg Services............................................ . & 138.7 & 138.7 & 132.3 & 127.4 & 7,900 \\
\hline Remedlal Services.................................................. & 143.8 & 150.0 & 150.0 & 150.0 & 2,400 \\
\hline 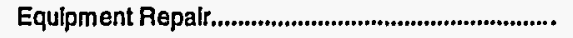 & 124.4 & 126.7 & 140.0 & 146.7 & 6,600 \\
\hline 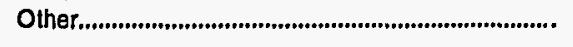 & 150.0 & 150.0 & 200.0 & 200.0 & 400 \\
\hline Subtotal or Index & 134.4 & 136.0 & 138.4 & 138.4 & 17,300 \\
\hline Total or Index & 213.3 & 220.7 & 217.5 & 214.6 & 95,500 \\
\hline
\end{tabular}

Table D6. Direct Annual Operating Costs and Indices for Primary Oil Production in Oklahoma (10 Wells Producing from 4,000 Feet by Rod Lift)

\begin{tabular}{|c|c|c|c|c|c|}
\hline \multirow[t]{2}{*}{ Component } & \multicolumn{4}{|c|}{ Index $(1976=100)$} & \multirow{2}{*}{$\begin{array}{c}\text { 1995* } \\
\text { Cost } \\
\text { (dollars) }\end{array}$} \\
\hline & 1992 & 1993 & 1994 & 1995 & \\
\hline \multicolumn{6}{|l|}{ Normal Dally Expense: } \\
\hline 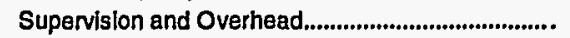 & 286.8 & 298.5 & 297.1 & 302.9 & 20,600 \\
\hline Lebor (pumper) .............................................................. & 210.8 & 223.0 & 223.0 & 223.0 & 16,500 \\
\hline 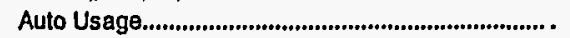 & 268.0 & 268.0 & 272.0 & 284.0 & 7,100 \\
\hline 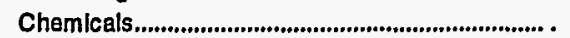 & 244.4 & 248.1 & 248.1 & 244.4 & 6,600 \\
\hline Fuel, Power \& Water............................................... & 292.4 & 321.2 & 292.4 & 248.5 & 16,400 \\
\hline Operallve Supplles......................................................... . & 233.3 & 233.3 & 233.3 & 233.3 & 1,400 \\
\hline Subtotal or Index & 259.8 & 273.7 & 266.5 & 257.9 & 68,600 \\
\hline \multicolumn{6}{|l|}{ Surface Maintenance, Repair \& Services: } \\
\hline 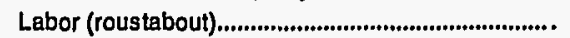 & 206.9 & 206.9 & 206.9 & 220.7 & 6,400 \\
\hline 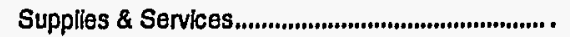 & 223.5 & 223.5 & 223.5 & 235.3 & 8,000 \\
\hline Equipment Usage....................................................... & 284.6 & 300.0 & 300.0 & 300.0 & 3,900 \\
\hline Subtotal or Index & 227.6 & 230.3 & 230.3 & 240.8 & 18,300 \\
\hline \multicolumn{6}{|l|}{ Subsurface Maintenance, Repair \& Services: } \\
\hline Workover Rig Services............................................ . & 160.6 & 160.6 & 154.3 & 150.0 & 14,100 \\
\hline Remedial Services..................................................... . & 154.2 & 154.2 & 154.2 & 158.3 & 3,800 \\
\hline Equlpment Repalr....................................................... & 122.0 & 124.0 & 126.0 & 146.0 & 7,300 \\
\hline 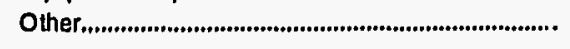 & 250.0 & 250.0 & 250.0 & 250.0 & 500 \\
\hline Subtotal or Index ${ }^{*+\ldots} \ldots$ & 149.4 & 150.0 & 147.1 & 151.2 & 25,700 \\
\hline Total or Index & 218,4 & 226.2 & 221.5 & 219.9 & 112,600 \\
\hline
\end{tabular}


Table D7. Direct Annual Operating Costs and Indices for Primary Oil Production in Oklahoma (10 Wells Producing from 8,000 Feat by Hydraulic Lift)

\begin{tabular}{|c|c|c|c|c|c|}
\hline \multirow[t]{2}{*}{ Component } & \multicolumn{4}{|c|}{ Index $(1976=100)$} & \multirow{2}{*}{$\begin{array}{c}1995^{*} \\
\text { Cost } \\
\text { (dollars) }\end{array}$} \\
\hline & 1992 & 1993 & 1994 & 1995 & \\
\hline \multicolumn{6}{|l|}{ Normal Daily Expense: } \\
\hline 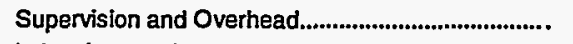 & 288.6 & 301.3 & 298.7 & 305.1 & 24,100 \\
\hline 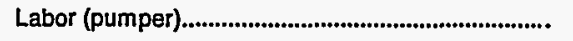 & 210.8 & 223.0 & 223.0 & 223.0 & 16,500 \\
\hline 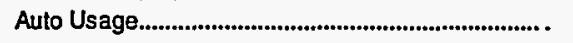 & 268.0 & 268.0 & 272.0 & 284.0 & 7,100 \\
\hline 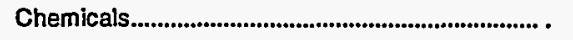 & 179.3 & 179.3 & 179.3 & 179.3 & 5,200 \\
\hline 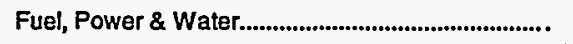 & 304.3 & 335.1 & 304.3 & 257.4 & 24,200 \\
\hline Operative Supplies............................................................. • & 287.5 & 300.0 & 300.0 & 312.5 & 2,500 \\
\hline Subtotal or Index ${ }^{* *}$ & 262.8 & 278.6 & 268.9 & 257.6 & 79,600 \\
\hline \multicolumn{6}{|l|}{ Subsurface Maintenance, Repair \& Services: } \\
\hline Labor (roustabout) & 206.9 & 206.9 & 206.9 & 220.7 & 6,400 \\
\hline Supplies \& Services......................................................... & 226.0 & 235.1 & 235.1 & 239.0 & 18,400 \\
\hline Equipment Usage................................................................. & 280.0 & 286.7 & 286.7 & 293.3 & 4,400 \\
\hline Subtotal or Index & 228.1 & 234.7 & 234.7 & 241.3 & 29,200 \\
\hline \multicolumn{6}{|l|}{ Subsurface Maintenance, } \\
\hline Workover Rig Services.............................................. . & 178.7 & 178.7 & 177.0 & 173.8 & 10,600 \\
\hline 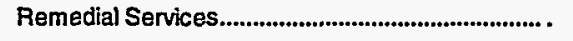 & 172.2 & 174.7 & 173.4 & 173.4 & 13,700 \\
\hline 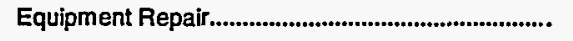 & 248.9 & 248.9 & 404.5 & 423.6 & 75,400 \\
\hline 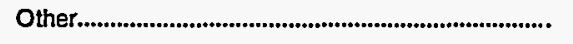 & 214.3 & 214.3 & 214.3 & 228.6 & 1,600 \\
\hline Subtotal or Index ${ }^{* *}$ & 216.3 & 216.9 & 301.5 & 311.7 & 101,300 \\
\hline Total or Index ${ }^{*}$ & 237.2 & 245.0 & 277.5 & 278.3 & 210,100 \\
\hline
\end{tabular}

Table D8. Direct Annual Operating Costs and Indices for Primary Oil Production in Oklahoma (10 Wells Producing from 12,000 Feet by Hydraulic Lift)

\begin{tabular}{|c|c|c|c|c|c|}
\hline \multirow{2}{*}{ Component } & \multicolumn{4}{|c|}{ Index $(1976=100)$} & \multirow{2}{*}{$\begin{array}{c}\text { 1995* } \\
\text { Cost } \\
\text { (dollars) }\end{array}$} \\
\hline & 1992 & 1993 & 1994 & 1995 & \\
\hline \multicolumn{6}{|l|}{ Normal Daily Expense: } \\
\hline 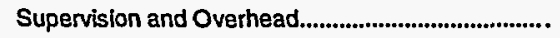 & 287.8 & 302.0 & 298.0 & 304.1 & 29,800 \\
\hline Labor (pumper) & 210.8 & 223.0 & 223.0 & 223.0 & 16,500 \\
\hline 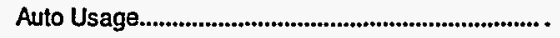 & 268.0 & 268.0 & 272.0 & 284.0 & 7,100 \\
\hline 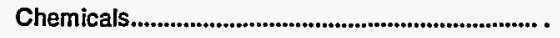 & 190.9 & 193.9 & 193.9 & 190.9 & 6,300 \\
\hline 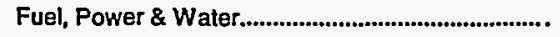 & 313.4 & 345.7 & 313.4 & 264.6 & 33,600 \\
\hline Operative Supplies........................................................ & 311.1 & 322.2 & 322.2 & 333.3 & 3,000 \\
\hline Subtotal or Index & 271.6 & 289.6 & 277.6 & 263.1 & 96,300 \\
\hline \multicolumn{6}{|l|}{ Surface Maintenance, Repair \& Services: } \\
\hline 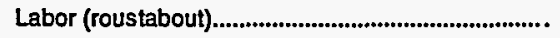 & 206.9 & 206.9 & 206.9 & 220.7 & 6,400 \\
\hline 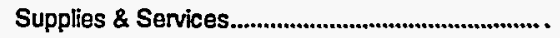 & 217.5 & 225.2 & 225.2 & 232.0 & 23,900 \\
\hline 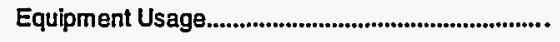 & 280.0 & 286.7 & 286.7 & 293.3 & 4,400 \\
\hline Subtotal or Index" & 221.8 & 227.9 & 227.9 & 236.1 & 34,700 \\
\hline \multicolumn{6}{|l|}{ Subsurface Maintenance, Repair \& Services: } \\
\hline Workover Rig Services.............................................. . & 182.4 & 182.4 & 180.2 & 176.9 & 16,100 \\
\hline 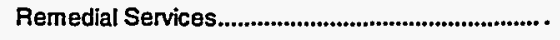 & 232.0 & 234.0 & 232.0 & 234.0 & 24,100 \\
\hline 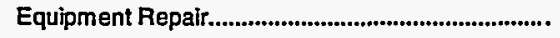 & 249.2 & 250.3 & 403.4 & 421.8 & 75,500 \\
\hline 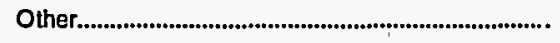 & 222.2 & 222.2 & 233.3 & 233.3 & 2,100 \\
\hline 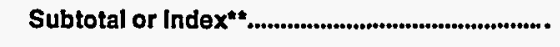 & 228.0 & 229.1 & 300.0 & 308.4 & 117,800 \\
\hline Total or index & 244.8 & 253.6 & 279.0 & 278.0 & 248,800 \\
\hline
\end{tabular}


Table E1. Lease Equipment Costs and Indices for Primary Oll Production in the Rocky Mountains (10 Wells Producing from 2,000 Feet by Rod Lift)

\begin{tabular}{|c|c|c|c|c|c|}
\hline \multirow{2}{*}{ Component } & \multicolumn{4}{|c|}{ Index $(1976=100)$} & \multirow{2}{*}{$\begin{array}{c}\text { 1995" } \\
\text { Cost } \\
\text { (dollars) }\end{array}$} \\
\hline & 1992 & 1993 & 1994 & 1995 & \\
\hline \multicolumn{6}{|l|}{ Producing Equipment: } \\
\hline 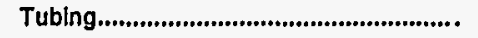 & 102.4 & 103.7 & 104.3 & 146.4 & 54,900 \\
\hline 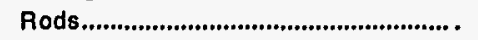 & 80.3 & 80.3 & 90.9 & 91.7 & 23,300 \\
\hline Pumps.................................................... . & 131.0 & 133.3 & 147.6 & 152.4 & 12,800 \\
\hline Pumplng Equipment............................. & 152.1 & 160.0 & 160.7 & 172.9 & 169,800 \\
\hline Subtotal or Index & 129.3 & 134.3 & 137.1 & 153.9 & 260,800 \\
\hline \multicolumn{6}{|l|}{ Gathering System: } \\
\hline 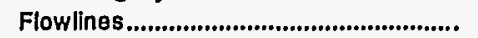 & 258.6 & 259.9 & 263.8 & 267.8 & 40,700 \\
\hline Manifold.................................................. & 260.2 & 258.6 & 261.7 & 264.7 & 35,200 \\
\hline Subtotal or Index & 259.3 & 259.3 & 262.8 & 266.3 & 75,900 \\
\hline \multicolumn{6}{|l|}{ Lease Equipment: } \\
\hline Producing Separator........................... . & 165.6 & 168.8 & 173.4 & 173.4 & 11,100 \\
\hline Test Separator......................................... & 190.1 & 193.1 & 189.1 & 188.1 & 19,000 \\
\hline Heater Treater......................................... . & 149.7 & 151.5 & 136.2 & 144.8 & 23,600 \\
\hline Storage Tanks..................................... & 183.9 & 186.9 & 189.9 & 194.9 & 65,300 \\
\hline Accessory Equipment.......................... & 206.8 & 211.6 & 213.6 & 221.1 & 32,500 \\
\hline 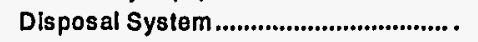 & 212.7 & 219.4 & 219.1 & 224.0 & 77,500 \\
\hline 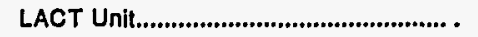 & 169.9 & 174.2 & 174.2 & 171.0 & 15,900 \\
\hline Electrificatlon........................................... & 243.6 & 245.2 & 244.4 & 247.3 & 59,600 \\
\hline Subtotal or Index & 197.5 & 201.3 & 200.2 & 204.4 & 304,500 \\
\hline 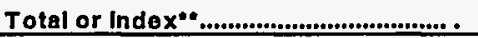 & 169.3 & 173.3 & 174.5 & 184.8 & 641,200 \\
\hline
\end{tabular}

Table E2. Lease Equipment Costs and Indices for Primary Oil Production in the Rocky Mountains (10 Wells Producing from 4,000 Feet by Rod Litt)

\begin{tabular}{|c|c|c|c|c|c|}
\hline \multirow{2}{*}{ Component } & \multicolumn{4}{|c|}{ Index $(1976=100)$} & \multirow{2}{*}{$\begin{array}{c}1995^{\star} \\
\text { Cost } \\
\text { (dollars) }\end{array}$} \\
\hline & 1992 & 1993 & 1994 & 1995 & \\
\hline \multicolumn{6}{|l|}{ Producing Equipment: } \\
\hline Tubing................................................... & 100.1 & 101.3 & 101.8 & 143.6 & 109,100 \\
\hline 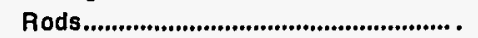 & 87.8 & 87.8 & 98.0 & 98.7 & 44,300 \\
\hline 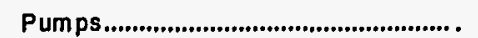 & 132.9 & 136.6 & 134.1 & 154.9 & 12,700 \\
\hline Pumplng Equipment.............................. & 131.6 & 137.3 & 137.9 & 159.5 & $\approx 96,500$ \\
\hline Subtotal or Index & 117.8 & 121.6 & 123.4 & 146.9 & 462,600 \\
\hline \multicolumn{6}{|l|}{ Gathering System: } \\
\hline 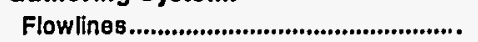 & 248.0 & 248.5 & 252.5 & 256.1 & 50,700 \\
\hline Manifold................................................ & 260.2 & 258.6 & 261.7 & 264.7 & 35,200 \\
\hline Subtotal or Index" ".................................. & 252.9 & 252.6 & 256.2 & 259.5 & 85,900 \\
\hline \multicolumn{6}{|l|}{ Lease Equipment: } \\
\hline Producling Separator............................ & 165.6 & 168.8 & 173.4 & 173.4 & 11,100 \\
\hline Test Separator....................................... & 190.1 & 193.1 & 189.1 & 188.1 & 19,000 \\
\hline Heater Treater...................................... & 149.7 & 151.5 & 136.2 & 144.8 & 23,600 \\
\hline 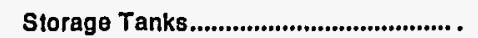 & 183.9 & 186.9 & 189.9 & 194.9 & 65,300 \\
\hline Accessory Equipm ent......................... & 206.8 & 211.6 & 213.6 & 221.1 & 32,500 \\
\hline Disposal System................................ & 215.1 & 222.2 & 221.9 & 226.8 & 79,600 \\
\hline 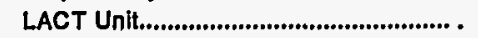 & 169.9 & 174.2 & 174.2 & 171.0 & 15,900 \\
\hline Electrification.......................................... & 242.9 & 244.6 & 242.5 & 245.6 & 70,500 \\
\hline Subtotal or Index" & 199.4 & 203.2 & 201.9 & 206.0 & 317,500 \\
\hline Total or Index & 151.7 & 155.2 & 156.2 & 172.4 & 866,000 \\
\hline
\end{tabular}

Energy Information Administration 
Table E3. Lease Equipment Costs and Indices for Primary Oil Production in the Rocky Mountains (10 Wells Producing from 8,000 Feet by Rod Litt)

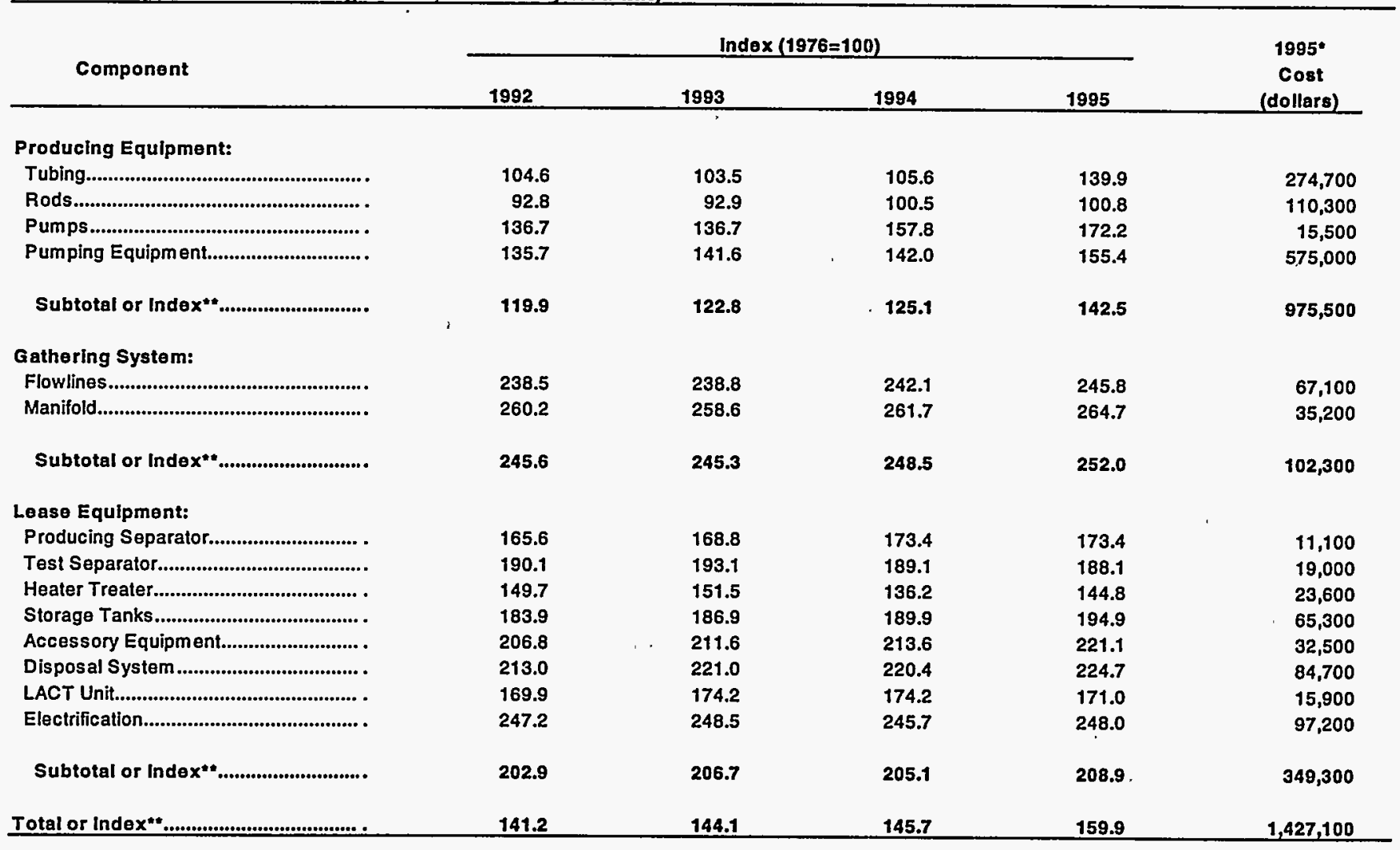

Table E4. Lease Equipment Costs and Indices for Primary Oil Production in the Rocky Mountains (10 Wells Producing from 12,000 Feet by Hydraulic Litt)

\begin{tabular}{|c|c|c|c|c|c|}
\hline \multirow{2}{*}{ Component } & \multicolumn{4}{|c|}{ Index $(1976=100)$} & \multirow{2}{*}{$\begin{array}{c}\text { 1995" } \\
\text { Cost } \\
\text { (dollars) }\end{array}$} \\
\hline & 1992 & 1993 & 1994 & 1995 & \\
\hline \multicolumn{6}{|l|}{ Producing Equipment: } \\
\hline 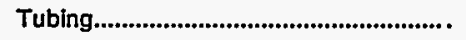 & 198.5 & 156.3 & 131.1 & 138.4 & 732,600 \\
\hline 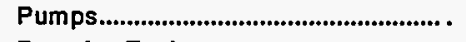 & 153.4 & 153.4 & 250.5 & 261.7 & 196,000 \\
\hline Pumping Equipment.............................. & 229.8 & 230.8 & 205.3 & 213.0 & 294,000 \\
\hline Subtotal or Index & 199.8 & 169.8 & 157.0 & 164.7 & $1,222,600$ \\
\hline \multicolumn{6}{|l|}{ Gathering System: } \\
\hline 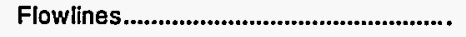 & 253.0 & 216.4 & 189.7 & 192.6 & 151,200 \\
\hline Manifold......................................................... & 260.2 & 258.6 & 261.7 & 264.7 & 35,200 \\
\hline Subtotal or Index & 254.0 & 222.5 & 200.1 & 203.1 & 186,400 \\
\hline \multicolumn{6}{|l|}{ Lease Equipment: } \\
\hline Producing Separator............................... . & 165.6 & 168.8 & 173.4 & 173.4 & 11,100 \\
\hline 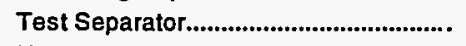 & 190.1 & 193.1 & 189.1 & 188.1 & 19,000 \\
\hline 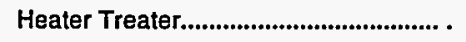 & 149.7 & 151.5 & 136.2 & 144.8 & 23,600 \\
\hline 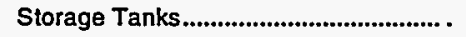 & 183.9 & 186.9 & 189.9 & 194.9 & 65,300 \\
\hline Accessory Equipment........................... & 206.8 & 211.6 & 213.6 & 221.1 & 32,500 \\
\hline Disposal System.................................. & 215.9 & 223.9 & 223.3 & 228.1 & 86,000 \\
\hline 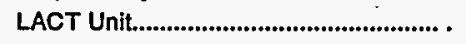 & 169.9 & 174.2 & 174.2 & 171.0 & 15,900 \\
\hline Electrification............................................. & 266.5 & 265.2 & 262.0 & 262.7 & 41,500 \\
\hline Subtotal or Index & 207.8 & 211.9 & 210.5 & 214.6 & 294,900 \\
\hline Total or Index ${ }^{* * \ldots}$ & 206.1 & 180.8 & 168.6 & 175.4 & $1,703,900$ \\
\hline
\end{tabular}


Table E5. Direct Annual Operating Costs and Indices for Primary Oil Production in the Rocky Mountalns (10 Wells Producing from 2,000 Feet by Rod Lift)

\begin{tabular}{|c|c|c|c|c|c|}
\hline \multirow[t]{2}{*}{ Component } & \multicolumn{4}{|c|}{ Index $(1976=100)$} & \multirow{2}{*}{$\begin{array}{c}\text { 1995* } \\
\text { Cost } \\
\text { (dollars) }\end{array}$} \\
\hline & 1992 & 1993 & 1994 & 1995 & \\
\hline \multicolumn{6}{|l|}{ Normal Dally Expense: } \\
\hline 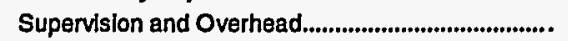 & 265.7 & 270.0 & 278.6 & 280.0 & 19,600 \\
\hline 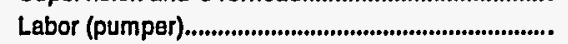 & 287.4 & 287.4 & 287.4 & 287.4 & 25,000 \\
\hline 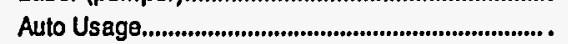 & 268.0 & 268.0 & 272.0 & 284.0 & 7,100 \\
\hline 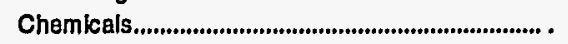 & 214.8 & 214.8 & 214.8 & 214.8 & 5,800 \\
\hline 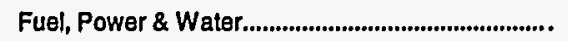 & 253.6 & 307.2 & 311.6 & 310.1 & 21,400 \\
\hline 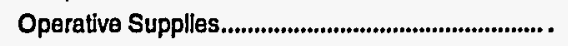 & 214.3 & 214.3 & 214.3 & 214.3 & 1,500 \\
\hline Subtotal or Index $=\ldots$ & 263.5 & 277.5 & 281.1 & 282.1 & 80,400 \\
\hline \multicolumn{6}{|l|}{ Surface MaIntenance, Repair \& Services: } \\
\hline Labor (roustabout),............................................................... & 207.3 & 212.2 & 212.2 & 212.2 & 8,700 \\
\hline Supplles \& Services............................................................ . & 212.9 & 206.5 & 200.0 & 193.5 & 6,000 \\
\hline 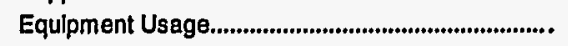 & 235.3 & 235.3 & 235.3 & 235.3 & 4,000 \\
\hline Subtotal or Index $=$ & 214.6 & 214.6 & 212.4 & 210.1 & 18,700 \\
\hline \multicolumn{6}{|l|}{ Subsurface Maintenance, Repair \& Services: } \\
\hline 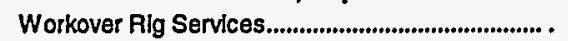 & 116.5 & 116.5 & 116.5 & 120.9 & 13,900 \\
\hline Remedial Services............................................................. . & 104.8 & 109.5 & 109.5 & 114.3 & 2,400 \\
\hline 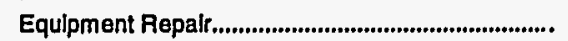 & 128.9 & 131.1 & 144.4 & 151.1 & 6,800 \\
\hline 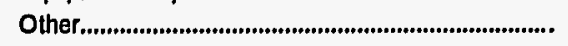 & 150.0 & 150.0 & 200.0 & 200.0 & 400 \\
\hline 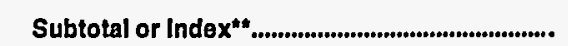 & 118.6 & 119.7 & 123.5 & 128.4 & 23,500 \\
\hline Total or Index" & 208.1 & 215.6 & 218.3 & 220.1 & 122,600 \\
\hline
\end{tabular}

Table E6. Direct Annual Operating Costs and Indices for Primary Oll Production In the Rocky Mountains (10 Wells Producing from 4,000 Feet by Rod Lift)

\begin{tabular}{|c|c|c|c|c|c|}
\hline \multirow{2}{*}{ Component } & \multicolumn{4}{|c|}{ Index $(1976=100)$} & \multirow{2}{*}{$\begin{array}{c}\text { 1995* } \\
\text { Cost } \\
\text { (dollars) }\end{array}$} \\
\hline & 1992 & 1993 & 1994 & 1995 & \\
\hline \multicolumn{6}{|l|}{ Normal Daily Expense: } \\
\hline 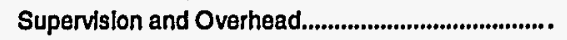 & 271.4 & 277.9 & 285.7 & 287.0 & 22,100 \\
\hline Labor (pumper) & 287.4 & 287.4 & 287.4 & 287.4 & 25,000 \\
\hline 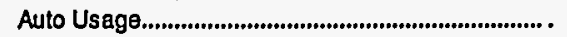 & 268.0 & 268.0 & 272.0 & 284.0 & 7,100 \\
\hline 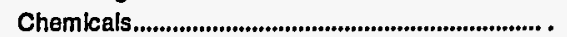 & 229.6 & 229.6 & 229.6 & 229.6 & 6,200 \\
\hline 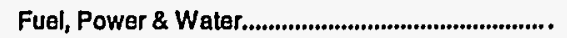 & 250.0 & 289.4 & 291.5 & 291.5 & 27,400 \\
\hline Operative Supplies....................................................... . & 214.3 & 228.6 & 228.6 & 228.6 & 1,600 \\
\hline Subtotal or Index" & 264.4 & 277.9 & 280.8 & 282.0 & 89,400 \\
\hline \multicolumn{6}{|l|}{ Surface Maintenance, Repair \& Services: } \\
\hline 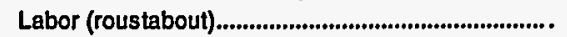 & 207.3 & 212.2 & 212.2 & 212.2 & 8,700 \\
\hline Supplles \& Services................................................... & 218.8 & 225.0 & 225.0 & 225.0 & 7,200 \\
\hline Equipment Usage & 233.3 & 233.3 & 233.3 & 233.3 & 4,200 \\
\hline Subtotal or Index & 216.5 & 220.9 & 220.9 & 220.9 & 20,100 \\
\hline \multicolumn{6}{|l|}{ Subsurface Malntenance, Repair \& Services: } \\
\hline Workover Rlg Services............................................ . & 113.9 & 113.9 & 113.9 & 118.5 & 17,900 \\
\hline Remedial Services................................................ . & 134.5 & 134.5 & 137.9 & 144.8 & 4,200 \\
\hline Equipment Repalt........................................................ & 122.0 & 124.0 & 126.0 & 146.0 & 7,300 \\
\hline 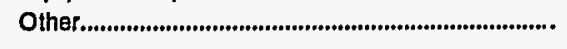 & 166.7 & 166.7 & 166.7 & 166.7 & 500 \\
\hline 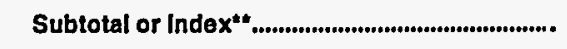 & 118.9 & 119.3 & 120.2 & 128.3 & 29,900 \\
\hline Total or Index" & 204.7 & 212.2 & 213.9 & 217.5 & 139,400 \\
\hline
\end{tabular}


Table E7. Direct Annual Operating Costs and Indices for Primary Oll Production in the Rocky Mountains (10 Wells Producing from 8,000 Feet by Rod Lift)

\begin{tabular}{|c|c|c|c|c|c|}
\hline \multirow[t]{2}{*}{ Component } & \multicolumn{4}{|c|}{ Index (1976=100) } & \multirow{2}{*}{$\begin{array}{c}\text { 1995* } \\
\text { Cost } \\
\text { (dollars) }\end{array}$} \\
\hline & 1992 & 1993 & 1994 & 1995 & \\
\hline \multicolumn{6}{|l|}{ Normal Daily Expense: } \\
\hline 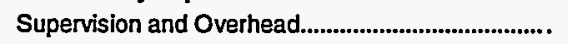 & 272.2 & 277.8 & 286.7 & 286.7 & 25,800 \\
\hline 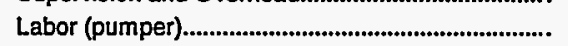 & 287.4 & 287.4 & 287.4 & 287.4 & 25,000 \\
\hline 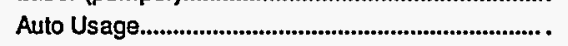 & 268.0 & 268.0 & 272.0 & 284.0 & 7,100 \\
\hline 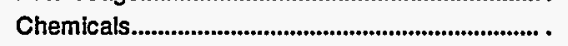 & 210.3 & 210.3 & 210.3 & 206.9 & 6,000 \\
\hline 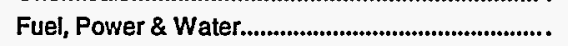 & 255.2 & 280.7 & 282.1 & 282.1 & 40,900 \\
\hline 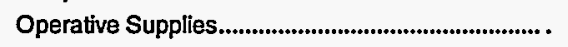 & 228.6 & 228.6 & 228.6 & 228.6 & 1,600 \\
\hline Subtotal or Index ${ }^{* *} \ldots$ & 263.4 & 274.4 & 277.3 & 277.8 & 106,400 \\
\hline \multicolumn{6}{|l|}{ Surface Maintenance, Repair \& Services: } \\
\hline 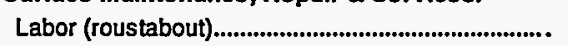 & 207.3 & 212.2 & 212.2 & 212.2 & 8,700 \\
\hline 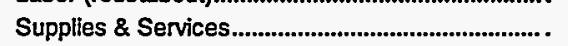 & 221.2 & 224.2 & 224.2 & 224.2 & 7,400 \\
\hline 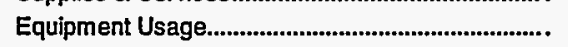 & 242.1 & 242.1 & 242.1 & 242.1 & 4,600 \\
\hline Subtotal or Index & 219.4 & 222.6 & 222.6 & 222.6 & 20,700 \\
\hline \multicolumn{6}{|l|}{ Subsurface Maintenance, Repair \& Services: } \\
\hline 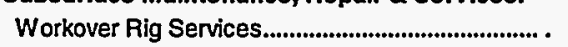 & 120.5 & 120.5 & 120.5 & 124.4 & 32,100 \\
\hline 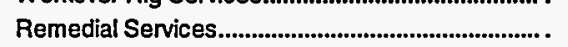 & 200.0 & 203.8 & 206.3 & 216.3 & 17,300 \\
\hline 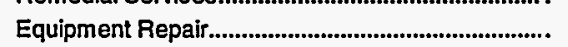 & 148.3 & 146.7 & 163.3 & 183.3 & 11,000 \\
\hline 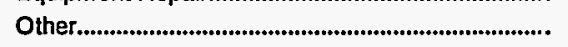 & 150.0 & 150.0 & 150.0 & 160.0 & 1,600 \\
\hline Subtotal or Index & 140.9 & 141.4 & 144.4 & 152.0 & 62,000 \\
\hline Total or Index ${ }^{* *} \ldots$ & 202.3 & 207.6 & 210.2 & 213.9 & 189,100 \\
\hline
\end{tabular}

Table E8. Direct Annual Operating Costs and Indices for Primary Oil Production in the Rocky Mountalns (10 Wells Producing from 12,000 Feet by Hydraullc Lift)

\begin{tabular}{|c|c|c|c|c|c|}
\hline \multirow[t]{2}{*}{ Component } & \multicolumn{4}{|c|}{ Index $(1976=100)$} & \multirow{2}{*}{$\begin{array}{c}1995^{\star} \\
\text { Cost } \\
\text { (dollars) }\end{array}$} \\
\hline & 1992 & 1993 & 1994 & 1995 & \\
\hline \multicolumn{6}{|l|}{ Normal Daily Expense: } \\
\hline 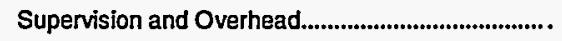 & 271.4 & 277.7 & 286.6 & 285.7 & 32,000 \\
\hline 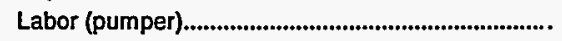 & 287.4 & 287.4 & 287.4 & 287.4 & 25,000 \\
\hline Auto Usage & 268.0 & 268.0 & 272.0 & 284.0 & 7,100 \\
\hline 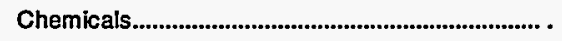 & 200.0 & 200.0 & 200.0 & 197.0 & 6,500 \\
\hline 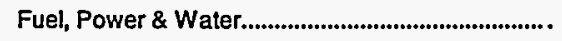 & 279.9 & 298.5 & 299.5 & 299.5 & 59,600 \\
\hline Operative Supplies........................................................ . & 220.0 & 230.0 & 230.0 & 230.0 & 2,300 \\
\hline Subtotal or Index $x^{* *}$ & 271.7 & 281.3 & 284.1 & 284.3 & 132,500 \\
\hline \multicolumn{6}{|l|}{ Surface Maintenance, Repair \& Services: } \\
\hline 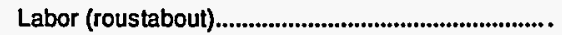 & 207.3 & 212.2 & 212.2 & 212.2 & 8,700 \\
\hline Supplies \& Services......................................................... & 228.3 & 238.3 & 238.3 & 238.3 & 14,300 \\
\hline 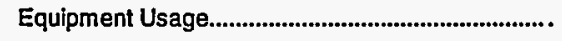 & 242.1 & 242.1. & 242.1 & 242.1 & 4,600 \\
\hline Subtotal or Index" & 223.3 & 230.0 & 230.0 & 230.0 & 27,600 \\
\hline \multicolumn{6}{|l|}{ Subsurface Maintenance, Repair \& Services: } \\
\hline Workover Rig Services.................................................. & 133.3 & 133.3 & 133.3 & 135.3 & 13,800 \\
\hline 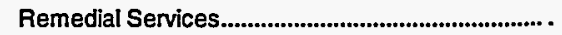 & 164.6 & 167.7 & 169.3 & 178.7 & 22,700 \\
\hline Equipment Repair.................................................................... & 249.7 & 250.3 & 403.9 & 421.8 & 75,500 \\
\hline 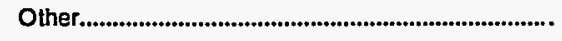 & 166.7 & 166.7 & 175.0 & 175.0 & 2,100 \\
\hline Subtotal or Index & 193.3 & 194.5 & 260.7 & 271.7 & 114,100 \\
\hline Total or Index $* * \ldots$ & 233.2 & 239.0 & 267.9 & 272.6 & 274,200 \\
\hline
\end{tabular}


Table F1. Lease Equipment Costs and Indices for Primary Oll Production in California (10 W ells Producing from 2,000 Feet by Rod Lift)

\begin{tabular}{|c|c|c|c|c|c|}
\hline \multirow{2}{*}{ Component } & \multicolumn{4}{|c|}{ Index $(1976=100)$} & \multirow{2}{*}{$\begin{array}{c}1995^{\circ} \\
\text { Cost } \\
\text { (dollars) }\end{array}$} \\
\hline & 1992 & 1993 & 1994 & 1995 & \\
\hline \multicolumn{6}{|l|}{ Produclng Equlpment: } \\
\hline 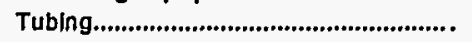 & 107.1 & 105.8 & 107.9 & 143.0 & 68,800 \\
\hline 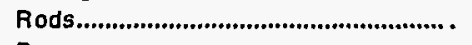 & 104.5 & 104.5 & 110.8 & 111.2 & 31,800 \\
\hline 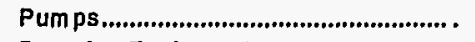 & 128.6 & 128.6 & 153.8 & 147.9 & 17,600 \\
\hline Pumping Equipment.............................. & 122.2 & 127.3 & 127.6 & 146.5 & 246,500 \\
\hline Subtotal or Index $* \star \ldots . . . . . . . . . . . . . . . . . . . . .$. & 117.7 & 120.8 & 123.3 & 142.0 & 364,700 \\
\hline \multicolumn{6}{|l|}{ Gathering System: } \\
\hline 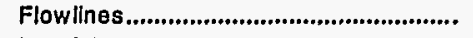 & 226.0 & 224.6 & 242.3 & 224.9 & 63,200 \\
\hline 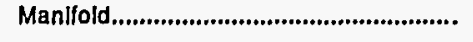 & 259.7 & 259.0 & 261.9 & 264.9 & 35,500 \\
\hline 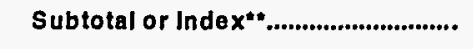 & 236.9 & 235.7 & 248.7 & 237.8 & 98,700 \\
\hline \multicolumn{6}{|l|}{ Lease Equipment: } \\
\hline Producling Separator............................. & 158.2 & 161.2 & 165.7 & 165.7 & 11,100 \\
\hline Test Separator............................................... & 190.1 & 193.1 & 189.1 & 188.1 & 19,000 \\
\hline Free water knockout............................... & 150.7 & 153.3 & 133.3 & 134.7 & 10,100 \\
\hline Heater Treater.......................................... & 229.3 & 233.7 & 231.7 & 243.2 & 143,500 \\
\hline 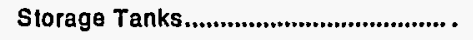 & 175.5 & 178.4 & 181.0 & 185.9 & 64,500 \\
\hline Accessory Equipment.......................... . & 206.8 & 210.9 & 212.9 & 220.4 & 32,400 \\
\hline Disposal System & 177.9 & 181.6 & 183.6 & 187.0 & 66,000 \\
\hline 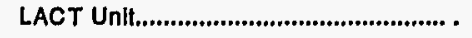 & 169.9 & 174.2 & 174.2 & 171.0 & 15,900 \\
\hline Electrification............................................ & 247.7 & 249.6 & 247.3 & 250.8 & 65,700 \\
\hline Subtotal or Index & 202.1 & 205.6 & 204.8 & 210.4 & 428,200 \\
\hline Total or Index & 161.7 & 164.7 & 166.7 & 177.6 & 891,600 \\
\hline
\end{tabular}

Table F2. Lease Equipment Costs and Indices for Primary Oil Production in Californla (10 Wells Producing from 4,000 Feet by Rod Lift)

\begin{tabular}{|c|c|c|c|c|c|}
\hline \multirow{2}{*}{ Component } & \multicolumn{4}{|c|}{ Index $(1976=100)$} & \multirow{2}{*}{ 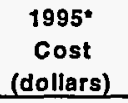 } \\
\hline & 1992 & 1993 & 1994 & 1995 & \\
\hline \multicolumn{6}{|l|}{ Produclng Equipment: } \\
\hline Tubing & 104.6 & 103.5 & 105.5 & 140.0 & 136,800 \\
\hline 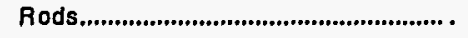 & 99.3 & 99.3 & 105.6 & 105.8 & 58,800 \\
\hline Pumps & 129.2 & 129.2 & 153.1 & 146.2 & 19,000 \\
\hline Pumping Equipm ent............................... & 104.0 & 108.7 & 108.9 & 124.3 & 338,400 \\
\hline Subtotal or Index & 104.3 & 107.0 & 109.1 & 126.1 & 553,000 \\
\hline \multicolumn{6}{|l|}{ Gathering System: } \\
\hline 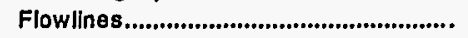 & 212.6 & 211.1 & 228.9 & 210.8 & 81,800 \\
\hline 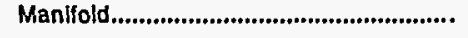 & 259.7 & 259.0 & 261.9 & 264.9 & 35,500 \\
\hline Subtotal or Index & 224.7 & 223.4 & 237.4 & 224.7 & 117,300 \\
\hline \multicolumn{6}{|l|}{ Lease Equipment: } \\
\hline Producing Separator.............................. . & 158.2 & 161.2 & 165.7 & 165.7 & 11,100 \\
\hline 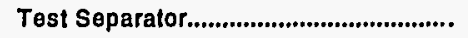 & 190.1 & 193.1 & 189.1 & 188.1 & 19,000 \\
\hline Free water knockout................................ & 150.7 & 153.3 & 133.3 & 134.7 & 10,100 \\
\hline Heater Treater......................................... & 229.3 & 233.7 & 231.7 & 243.2 & 143,500 \\
\hline 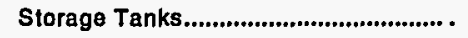 & 175.5 & 178.4 & 181.0 & 185.9 & 64,500 \\
\hline Accessory Equipment............................ & 206.8 & 210.9 & 212.9 & 220.4 & 32,400 \\
\hline Dlsposal System .................................... & 174.8 & 178.3 & 180.8 & 183.5 & 67,700 \\
\hline 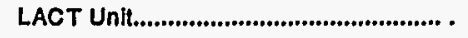 & 169.9 & 174.2 & 174.2 & 171.0 & 15,900 \\
\hline 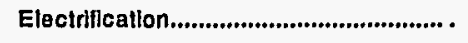 & 226.5 & 228.0 & 225.9 & 229.3 & 75,200 \\
\hline Subtotal or Index & 199.5 & 202.8 & 202.2 & 207.6 & 439,400 \\
\hline Total or Index" & 141.9 & 144.5 & 146.7 & 158.0 & $1,109,700$ \\
\hline
\end{tabular}

Energy Information Administration 
Table F3. Lease Equipment Costs and Indices for Primary Oil Production in California : (10 Wells Producing from 8,000 Feet by Hydraulic Lift)

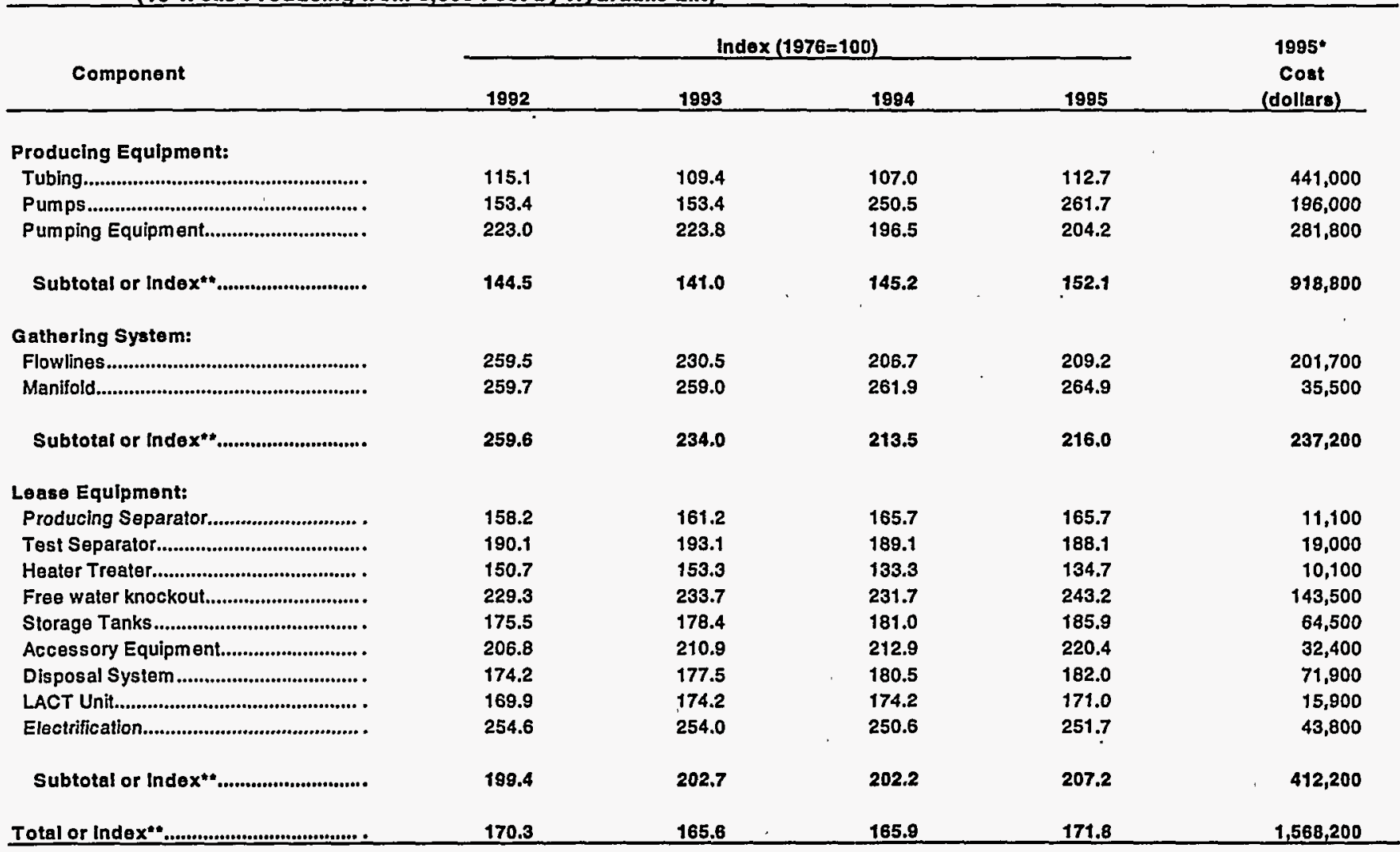

Table F4. Lease Equipment Costs and Indices for Primary Oil Production in Calfornia (10 Wells Producing from 12,000 Feat by Hydraullc Lift)

\begin{tabular}{|c|c|c|c|c|c|}
\hline \multirow{2}{*}{ Component } & \multirow[b]{2}{*}{1992} & \multicolumn{2}{|c|}{ Index $(1976=100)$} & \multirow[b]{2}{*}{1995} & \multirow{2}{*}{$\begin{array}{c}\text { 1895" } \\
\text { Cost } \\
\text { (dollars) }\end{array}$} \\
\hline & & 1993 & 1994 & & \\
\hline \multicolumn{6}{|l|}{ Producing Equlpment: } \\
\hline 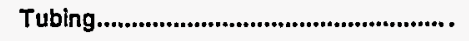 & 179.3 & 141.1 & 118.4 & 125.0 & 732,200 \\
\hline 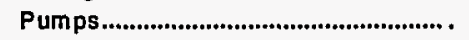 & 153.4 & 153.4 & 250.5 & 261.7 & 196,000 \\
\hline Pumping Equipment................................ & 229.4 & 230.2 & 202.9 & 210.8 & 322,800 \\
\hline Subtotal or Index*\#............................ & 186.3 & 159.0 & 146.4 & 153.7 & $1,251,000$ \\
\hline \multicolumn{6}{|l|}{ Gathering System: } \\
\hline 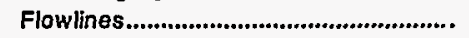 & 259.5 & 230.5 & 206.7 & 209.2 & 201,700 \\
\hline 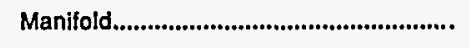 & 259.7 & 259.0 & 261.9 & 264.9 & 35,500 \\
\hline Subtotal or Index & 259.6 & 234.0 & 213.5 & 216.0 & 237,200 \\
\hline \multicolumn{6}{|l|}{ Leaso Equipment: } \\
\hline Producing Separator.............................. . & 158.2 & 161.2 & 165.7 & 165.7 & 11,100 \\
\hline 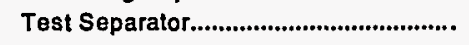 & 190.1 & 193.1 & 189.1 & 188.1 & 19,000 \\
\hline Heater Treater............................................ . & 150.7 & 153.3 & 133.3 & 134.7 & 10,100 \\
\hline Free water knockout............................... & 229.3 & 233.7 & 231.7 & 243.2 & 143,500 \\
\hline 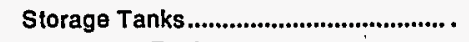 & 175.5 & 178.4 & 181.0 & 185.9 & 64,500 \\
\hline Accessory Equipment........................... & 206.8 & 210.9 & 212.9 & 220.4 & 32,400 \\
\hline Disposal System & 174.2 & 177.5 & 180.5 & 182.0 & 71,900 \\
\hline 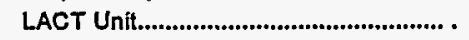 & 169.9 & 174.2 & 174.2 & 171.0 & 15,900 \\
\hline Electrification............................................ & 260.2 & 259.7 & 258.7 & 258.3 & , 53,200 \\
\hline Subtotal or Index & 200.8 & 204.1 & 203.8 & 208.6 & 421,600 \\
\hline 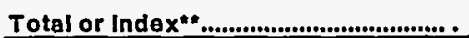 & 196.1 & 174.4 & 163.3 & 169.6 & $1,909,800$ \\
\hline
\end{tabular}


Table F5. Direct Annual Operating Costs and Indices for Primary Oll Production in Callfornla (10 Wells Producing from 2,000 Feet by Rod Lift)

\begin{tabular}{|c|c|c|c|c|c|}
\hline \multirow[t]{2}{*}{ Component } & \multicolumn{4}{|c|}{ Index $(1976=100)$} & \multirow{2}{*}{$\begin{array}{c}\text { 1995' } \\
\text { Cost } \\
\text { (dollars) }\end{array}$} \\
\hline & 1992 & 1993 & 1994 & 1995 & \\
\hline \multicolumn{6}{|l|}{ Normal Dally Expenso: } \\
\hline Supervision and Overhead........................................ & 295.0 & 305.0 & 308.3 & 321.7 & 19,300 \\
\hline 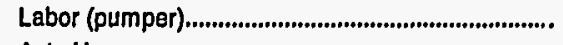 & 276.0 & 276.0 & 276.0 & 276.0 & 26,500 \\
\hline 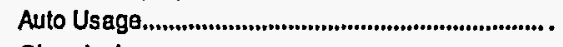 & 268.0 & 268.0 & 272.0 & 284.0 & 7,100 \\
\hline 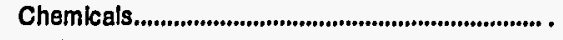 & 226.3 & 226.3 & 226.3 & 221.1 & 4,200 \\
\hline 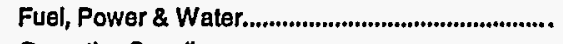 & 427.4 & 430.1 & 482.3 & 431.9 & 48,800 \\
\hline Operative Supplles...................................................... & 225.0 & 225.0 & 225.0 & 225.0 & 1,800 \\
\hline Subtotal or Index & 328.0 & 330.8 & 350.2 & 335.5 & 107,700 \\
\hline \multicolumn{6}{|l|}{ Surface Maintenance, Repair \& Services: } \\
\hline Labor (roustabout) & 200.0 & 200.0 & 200.0 & 200.0 & 10,800 \\
\hline Supplies \& Services .............................................. & 231.0 & 234.5 & 231.0 & 234.5 & 6,800 \\
\hline 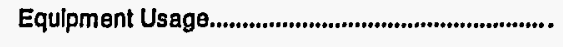 & 242.9 & 250.0 & 221.4 & 221.4 & 3,100 \\
\hline Subtotal or Index & 215.5 & 217.5 & 212.4 & 213.4 & 20,700 \\
\hline Subsurface Maintenance, Repalr \& Services: & & & & & \\
\hline Workover Rig Services............................................... . & 170.5 & 170.5 & 170.5 & 172.1 & 10,500 \\
\hline Remedlal Services......................................................... . & 142.9 & 142.9 & 142.9 & 157.1 & 1,100 \\
\hline Equipment Repair.......................................................... & 140.7 & 140.7 & 164.4 & 164.4 & 9,700 \\
\hline 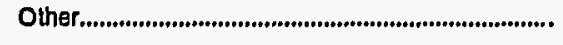 & 150.0 & 150.0 & 150.0 & 150.0 & 300 \\
\hline Subtotal or Index & 155.0 & 155.0 & 165.9 & 167.4 & 21,600 \\
\hline 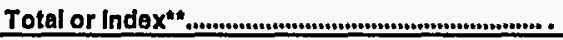 & 267.3 & 269.3 & 282.3 & 274.2 & 150,000 \\
\hline
\end{tabular}

Table F6. Direct Annual Operating Costs and Indices for Primary Oll Production in Californla (10 Wells Producing from 4,000 Feet by Rod Lift)

\begin{tabular}{|c|c|c|c|c|c|}
\hline \multirow{2}{*}{ Component } & \multicolumn{4}{|c|}{ Index $(1976=100)$} & \multirow{2}{*}{$\begin{array}{c}\text { 1995 } \\
\text { Cost } \\
\text { (dollars) }\end{array}$} \\
\hline & 1992 & 1993 & 1994 & 1995 & \\
\hline \multicolumn{6}{|l|}{ Normal Dally Expense: } \\
\hline Supervision and Overhead...................................... & 292.6 & 302.9 & 307.4 & 319.1 & 21,700 \\
\hline 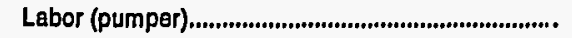 & 276.0 & 276.0 & 276.0 & 276.0 & 26,500 \\
\hline 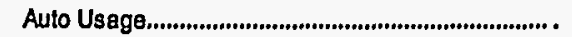 & 268.0 & 268.0 & 272.0 & 284.0 & 7,100 \\
\hline 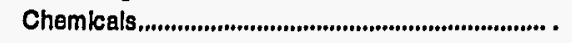 & 223.8 & 223.8 & 223.8 & 219.0 & $4,600^{-}$ \\
\hline Fuel, Power \& Water............................................... & 480.6 & 483.4 & 533.1 & 483.4 & 84,600 \\
\hline Operative Supplles........................................................ . & 225.0 & 225.0 & 225.0 & 225.0 & 1,800 \\
\hline Subtotal or Index & 365.6 & 368.7 & 391.9 & 372.3 & 146,300 \\
\hline \multicolumn{6}{|l|}{ Surface Maintenance, Repair \& Services: } \\
\hline Labor (roustabout) .................................................... & 200.0 & 200.0 & 200.0 & 200.0 & 10,800 \\
\hline Supplies \& Services................................................ . & 225.8 & 229.0 & 225.8 & 225.8 & 7,000 \\
\hline 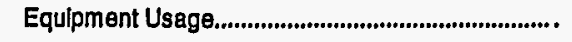 & 240.0 & 246.7 & 220.0 & 220.0 & 3,300 \\
\hline Subtotal or Index & 214.0 & 216.0 & 211.0 & 211.0 & 21,100 \\
\hline \multicolumn{6}{|l|}{ Subsurface Malntenance, Repalt \& Servlces: } \\
\hline Workover Rlg Services............................................. . & 171.0 & 171.0 & 171.0 & 173.0 & 17,300 \\
\hline Remedial Services................................................... & 176.9 & 184.6 & 184.6 & 184.6 & 2,400 \\
\hline Equipment Repair.................................................... & 137.0 & 137.0 & 156.8 & 158.0 & 12,800 \\
\hline 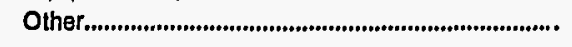 & 166.7 & 166.7 & 166.7 & 166.7 & 500 \\
\hline 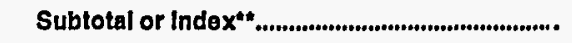 & 157.4 & 157.9 & 166.0 & 167.5 & 33,000 \\
\hline Total or Index & 284.2 & 286.4 & 301.2 & 290.4 & 200,400 \\
\hline
\end{tabular}


Table F7. Direct Annual Operating Costs and Indices for Primary Oll Production in California ( 10 Wells Producing from 8,000 Feet by Hydraulic Lift)

\begin{tabular}{|c|c|c|c|c|c|}
\hline \multirow{3}{*}{ Component } & \multirow{2}{*}{\multicolumn{4}{|c|}{ Index $(1976=100)$}} & \multirow{3}{*}{$\begin{array}{c}\text { 1995* } \\
\text { Cost } \\
\text { (dollars) }\end{array}$} \\
\hline & & & & & \\
\hline & 1992 & 1993 & 1994 & 1995 & \\
\hline \multicolumn{6}{|l|}{ Normal Daily Expense: } \\
\hline 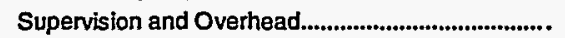 & 293.7 & 305.1 & 308.9 & 320.3 & 25,300 \\
\hline Labor (pumper) & 276.0 & 276.0 & 276.0 & 276.0 & 26,500 \\
\hline 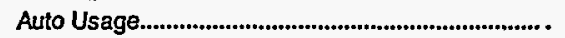 & 268.0 & 268.0 & 272.0 & 284.0 & 7,100 \\
\hline 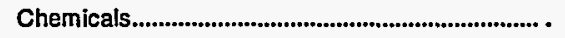 & 220.0 & 223.3 & 220.0 & 220.0 & 6,600 \\
\hline 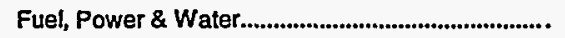 & 517.8 & 519.5 & 570.5 & 523.7 & 188,000 \\
\hline Operative Supplies........................................................... & 209.1 & 209.1 & 209.1 & 209.1 & 2,300 \\
\hline 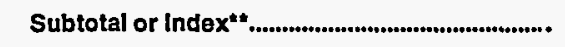 & 418.7 & 421.3 & 452.3 & 426.3 & 255,800 \\
\hline \multicolumn{6}{|l|}{ Surface Maintenance, Repair \& Services: } \\
\hline Labor (roustabout) & 200.0 & 200.0 & 200.0 & 200.0 & 10,800 \\
\hline Supplies \& Services................................................. & 260.3 & 267.6 & 264.7 & 266.2 & 18,100 \\
\hline Equipment Usage.................................................... & 250.0 & 262.5 & 231.3 & 231.3 & 3,700 \\
\hline Subtotal or Index & 235.5 & 240.6 & 235.5 & 236.2 & 32,600 \\
\hline \multicolumn{6}{|l|}{ Subsurface Maintenance, Repair \& Services: } \\
\hline Workover Rig Services............................................... . & 184.2 & 184.2 & 184.2 & 189.5 & 3,600 \\
\hline 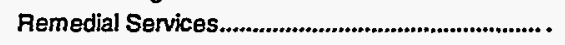 & 212.3 & 214.0 & 214.0 & 219.3 & 12,500 \\
\hline 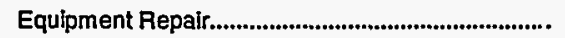 & 235.0 & 234.0 & 368.9 & 392.2 & 40,400 \\
\hline 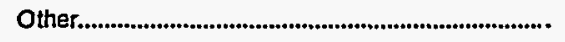 & 166.7 & 166.7 & 166.7 & 166.7 & 1,500 \\
\hline 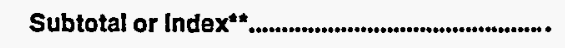 & 219.7 & 219.7 & 293.6 & 308.5 & 58,000 \\
\hline Total or Index & 351.0 & 353.5 & 387.8 & 374.1 & 346,400 \\
\hline
\end{tabular}

Table F8. Direct Annual Operating Costs and Indices for Primary Oil Production in California (10 Wells Producing from 12,000 Feet by Hydraulic Lift)

\begin{tabular}{|c|c|c|c|c|c|}
\hline \multirow[t]{2}{*}{ Component } & \multicolumn{4}{|c|}{ Index $(1976=100)$} & \multirow{2}{*}{$\begin{array}{c}\text { 1995* } \\
\text { Cost } \\
\text { (dollars) }\end{array}$} \\
\hline & 1992 & 1993 & 1994 & 1995 & \\
\hline \multicolumn{6}{|l|}{ Normal Daily Expense: } \\
\hline 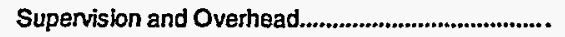 & 293.9 & 305.1 & 308.2 & 321.4 & 31,500 \\
\hline Labor (pumper) & 276.0 & 276.0 & 276.0 & 276.0 & 26,500 \\
\hline 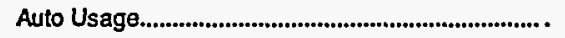 & 268.0 & 268.0 & 272.0 & 284.0 & $7,+00$ \\
\hline 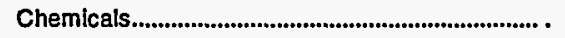 & 211.4 & 211.4 & 208.6 & 208.6 & 7,300 \\
\hline 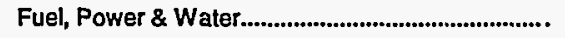 & 515.8 & 518.0 & 564.0 & 519.2 & 288,700 \\
\hline 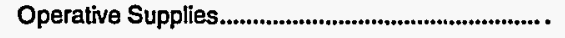 & 200.0 & 200.0 & 200.0 & 200.0 & 2,400 \\
\hline Subtotal or Index & 436.3 & 439.1 & 470.6 & 442.2 & 363,500 \\
\hline \multicolumn{6}{|l|}{ Surface Maintenance, Repair \& Services: } \\
\hline Labor (roustabout) & 200.0 & 200.0 & 200.0 & 200.0 & 10,800 \\
\hline Supplies \& Services................................................... . & 239.5 & 246.5 & 244.2 & 245.3 & 21,100 \\
\hline 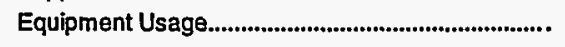 & 250.0 & 262.5 & 231.3 & 231.3 & 3,700 \\
\hline Subtotal or Index"*.............................................. & 226.9 & 232.1 & 227.6 & 228.2 & 35,600 \\
\hline \multicolumn{6}{|l|}{ Subsurface Maintenance, Repair \& Services: } \\
\hline Workover Rig Services............................................. . & 192.9 & 192.9 & 192.9 & 192.9 & 5,400 \\
\hline 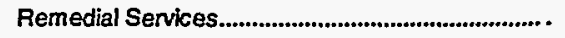 & 177.1 & 178.1 & 179.0 & 183.8 & 19,300 \\
\hline 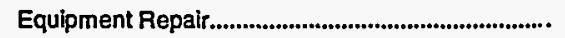 & 254.7 & 255.3 & 411.7 & 430.2 & 77,000 \\
\hline 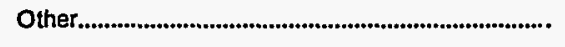 & 153.8 & 153.8 & 153.8 & 153.8 & 2,000 \\
\hline Subtotal or Index & 220.3 & 220.9 & 307.4 & 319.1 & 103,700 \\
\hline Total or Index & 357.3 & 359.9 & 400.8 & 385.9 & 502,800 \\
\hline
\end{tabular}


Table G1. Annual Operating Costs and Indices for a 12-Slot Platform in the Gulf of Mexico 100-Foot Water Depth

\begin{tabular}{|c|c|c|c|c|c|}
\hline \multirow[t]{2}{*}{ Component } & \multicolumn{4}{|c|}{ Index $(1976=100)$} & \multirow{2}{*}{$\begin{array}{c}1995^{\star} \\
\text { Cost } \\
\text { (dollars) }\end{array}$} \\
\hline & 1992 & 1993 & 1994 & 1995 & \\
\hline 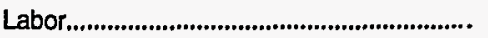 & 261.9 & 261.9 & 272.4 & 272.4 & 629,300 \\
\hline Supenision & 262.4 & 262.4 & 272.8 & 272.8 & 94,400 \\
\hline 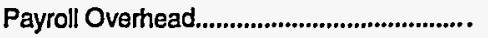 & 349.3 & 349.3 & 363.2 & 363.2 & 289,500 \\
\hline Food Expense.................................................. . & 146.7 & 153.8 & 183.7 & 189.7 & 75,500 \\
\hline Labor Transportation................................... & 249.1 & 259.4 & 259.4 & 259.4 & 458,600 \\
\hline Surface Equipment....................................... & 224.4 & 227.7 & 224.6 & 229.3 & 113,500 \\
\hline Operating Supplies........................................ & 224.2 & 227.3 & 224.2 & 229.3 & 22,700 \\
\hline 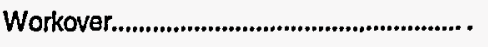 & 264.9 & 282.8 & 286.4 & 313.6 & 857,500 \\
\hline Communications........................................... . & 483.7 & 479.1 & 479.1 & 479.1 & 20,600 \\
\hline 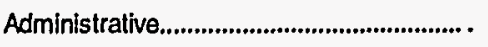 & 254.7 & 255.3 & 263.0 & 264.0 & 306,200 \\
\hline 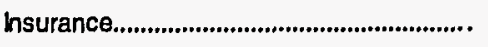 & 107.2 & 123.3 & 144.9 & 144.9 & 310,700 \\
\hline Total or Index & 234.0 & 242.7 & 252.0 & 258.5 & $3,178,500$ \\
\hline
\end{tabular}

Table G2. Annual Operating Costs and Indices for a 12-Slot Platform in the Gulf of Mexico 300-Foot Water Depth

\begin{tabular}{|c|c|c|c|c|c|}
\hline \multirow[t]{2}{*}{ Component } & \multicolumn{4}{|c|}{ Index $(1976=100)$} & \multirow{2}{*}{$\begin{array}{c}\text { 1995* } \\
\text { Cost } \\
\text { (dollars) }\end{array}$} \\
\hline & 1992 & 1993 & 1994 & 1995 & \\
\hline 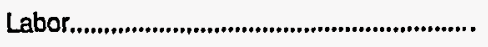 & 261.9 & 261.9 & 272.4 & 272.4 & 629,300 \\
\hline Supervision.................................................. & 262.4 & 262.4 & 272.8 & 272.8 & 94,400 \\
\hline 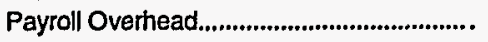 & 349.3 & 349.3 & 363.2 & 363.2 & 289,500 \\
\hline Food Expense.,.......................................... . & 146.7 & 153.8 & 183.7 & 189.7 & 75,500 \\
\hline Labor Transportation.................................. & 253.8 & 263.2 & 263.2 & 263.2 & 505,400 \\
\hline Surface Equipment........................................ & 224.6 & 227.8 & 224.6 & 229.4 & 114,700 \\
\hline Operating Supplies,..................................... & 225.0 & 228.0 & 225.0 & 229.0 & 22,900 \\
\hline 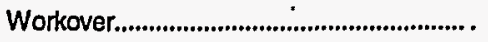 & 269.4 & 288.3 & 291.8 & 319.5 & 913,800 \\
\hline Communications........................................... . & 93.8 & 93.1 & 93.1 & 93.1 & 28,400 \\
\hline 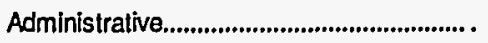 & 254.5 & 255.1 & 262.8 & 263.8 & 306,800 \\
\hline 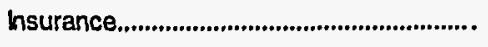 & 106.4 & 122.4 & 143.9 & 143.9 & 350,300 \\
\hline Total or Index & 209.5 & 217.6 & 226.0 & 231.9 & $3,331,000$ \\
\hline
\end{tabular}

Table G3. Annual Operating Costs and Indices for a 18-Slot Platform in the Gulf of Mexico

\begin{tabular}{|c|c|c|c|c|c|}
\hline \multirow[t]{2}{*}{ Component } & \multicolumn{4}{|c|}{ Index $(1976=100)$} & \multirow{2}{*}{$\begin{array}{c}1995^{\star} \\
\text { Cost } \\
\text { (dollars) }\end{array}$} \\
\hline & 1992 & 1993 & 1994 & 1995 & \\
\hline 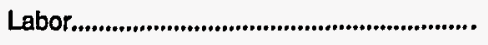 & 253.6 & 253.6 & 263.8 & 263.8 & 693,700 \\
\hline Supervision,............................................... & 254.1 & 254.1 & 264.2 & 264.2 & 104,100 \\
\hline Payroll Overhead............................................ & 337.9 & 337.9 & 351.4 & 351.4 & 319,100 \\
\hline 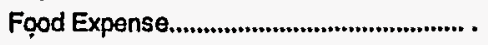 & 146.6 & 153.6 & 183.5 & 189.5 & 86,200 \\
\hline Labor Transportation..................................... & 249.1 & 259.4 & 259.4 & 259.4 & 458,600 \\
\hline Surface Equipment..................................... & 202.9 & 205.8 & 203.1 & 207.2 & 114,600 \\
\hline Operating Supplies......................................... & 201.8 & 205.4 & 202.7 & 206.3 & 22,900 \\
\hline 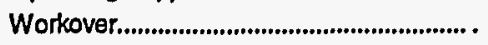 & 269.0 & 287.2 & 290.9 & 318.5 & $1,286,200$ \\
\hline 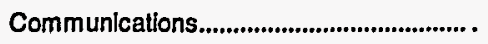 & 169.1 & 167.5 & 167.5 & 167.5 & 20,600 \\
\hline 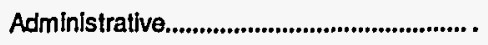 & 244.0 & 244.6 & 252.2 & 253.0 & 332,500 \\
\hline 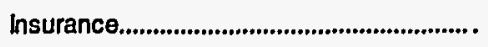 & 106.3 & 122.3 & 143.6 & 143.6 & 455,500 \\
\hline Total or Index & 204.1 & 212.8 & 221.5 & 228.4 & $3,894,000$ \\
\hline
\end{tabular}


Table G4. Annual Operating Costs and Indices for a 18-Slot Platform in the Gulf of Mexico 300-Foot Water Depth

\begin{tabular}{|c|c|c|c|c|c|c|c|}
\hline \multirow[t]{2}{*}{ Component } & & \multicolumn{5}{|c|}{ Index $(1976=100)$} & \multirow{2}{*}{$\begin{array}{c}1995^{\star} \\
\text { Cost } \\
\text { (dollars) }\end{array}$} \\
\hline & & 1992 & . & 1993 & 1994 & 1995 & \\
\hline 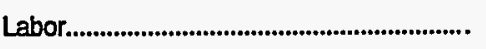 & & 253.6 & & 253.6 & 263.8 & 263.8 & 693,700 \\
\hline 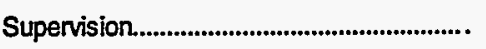 & . & 254.1 & & 254.1 & 264.2 & 264.2 & 104,100 \\
\hline Payroll Overhead.......................................... & & 338.3 & & 338.3 & 351.8 & 351.8 & 319,100 \\
\hline Food Expens & & 146.6 & : & 153.6 & 183.5 & 189.5 & 86,200 \\
\hline Labor Transportation.................................... . & & 203.6 & & 213.1 & 213.1 & 213.1 & 409,200 \\
\hline Surface Equipment....................................... & & 200.7 & & 203.6 & 200.9 & 205.0 & 114,600 \\
\hline 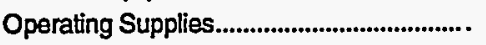 & & 200.0 & & 203.6 & 200.9 & 204.5 & 22,900 \\
\hline 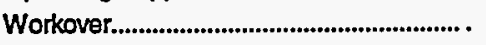 & & 273.3 & & 292.5 & 296.0 & 324.1 & $1,370,600$ \\
\hline Communications........................................... & - & 93.8 & & 93.1 & 93.1 & 93.1 & 28,400 \\
\hline 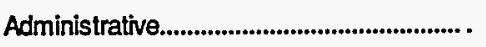 & : & 243.2 & & 243.9 & 251.4 & 252.3 & 332,500 \\
\hline hsurance........................................................... & & 105.3 & & 121.1 & 142.3 & 142.3 & 486,200 \\
\hline Total or Index & & 197.9 & & 206.8 & 215.3 & 222.3 & $3,967,500$ \\
\hline
\end{tabular}

Table G5. Annual Operating Costs and Indices for a 18-Slot Platform in the Gulf of Mexico 600-Foot Water Depth

\begin{tabular}{|c|c|c|c|c|c|}
\hline \multirow[t]{2}{*}{ Component } & \multicolumn{4}{|c|}{ Index $(1976=100)$} & \multirow{2}{*}{$\begin{array}{c}\text { 1995" } \\
\text { Cost } \\
\text { (dollars) }\end{array}$} \\
\hline & 1992 & 1993 & 1994 & 1995 & \\
\hline 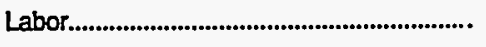 & 253.6 & 253.6 & 263.8 & 263.8 & 693,700 \\
\hline Supervision..................................................... & 254.1 & 254.1 & 264.2 & 264.2 & 104,100 \\
\hline Payroll Overhead.............................................. & 338.3 & 338.3 & 351.8 & 351.8 & 319,100 \\
\hline Food Expense................................................ . & 146.6 & 153.6 & 183.5 & 189.5 & 86,200 \\
\hline Labor Transportation...................................... & 246.8 & 255.7 & 255.7 & 255.7 & 523,080 \\
\hline Surface Equipment..................................... & 226.8 & 230.0 & 227.0 & 231.6 & 130,600 \\
\hline Operating Supplies.......................................... & 226.5 & 229.2 & 226.5 & 231.0 & 26,100 \\
\hline 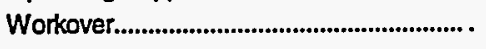 & 263.7 & 282.2 & 286.6 & 313.5 & $1,405,200$ \\
\hline Communications............................................. & 88.8 & 88.3 & 88.3 & 88.3 & 32,300 \\
\hline 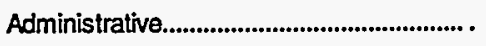 & 248.4 & 249.1 & 256.5 & 257.5 & 340,200 \\
\hline Insurance.......................................................... & 111.8 & 128.6 & 151.1 & 151.1 & 781,700 \\
\hline Total or Index & 193.2 & 202.7 & 212.6 & 218.9 & $4,442,280$ \\
\hline
\end{tabular}




\section{Section II}

Appendices $\mathrm{H}$ Through $\mathbf{M}$

Costs and Indices for Domestic Gas Field Equipment and Production Operations 


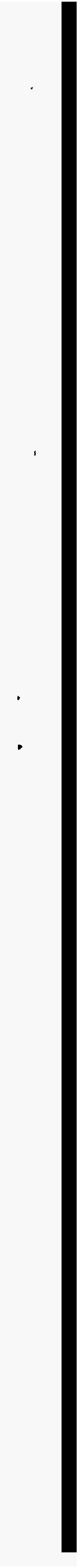




\section{Appendices H Through M}

\section{Costs and Indices for Domestic Gas Field Equipment and Production Operations}

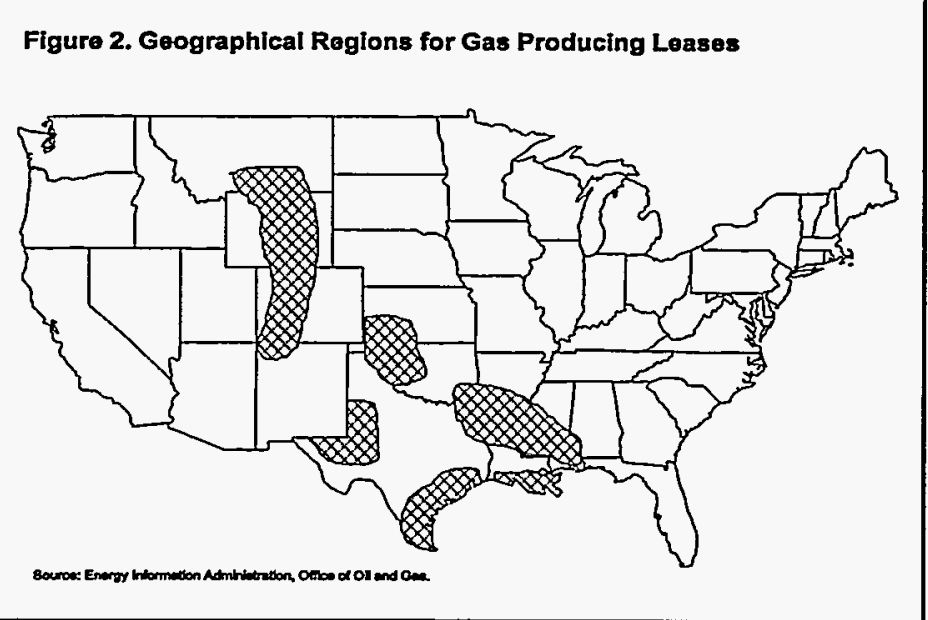

Appendices $\mathrm{H}$ through $\mathrm{M}$ contain details for gas leases. A detailed breakdown of 1995 costs and indices for 1992 through 1995 is shown in each of the gas lease tables. The tables are arranged by region with each region identified by an alpha character. Each table within the appendix is for a different depth. For example, Table H1 contains equipping cost data for west Texas gas leases at 2,000-foot depths; Table $\mathrm{H} 2$ contains equipping cost data for gas leases at 4,000-foot depths; Table H5 contains equipping cost data for 16,000 -foot wells. Tables H6 through $\mathrm{H} 10$ contain operating costs for gas wells at $2,000,4,000,8,000,12,000$ and 16,000-foot depths, respectively. Each table is further divided into costs associated with different flow rates. For example, Table $\mathrm{H} 1$ has equipping costs for production rates of 50 and 250 thousand cubic feet per day only. Table H11 is a typical equipment list for a 12,000 -foot gas well producing 1 million cubic feet per day in west Texas.

The remaining tables of costs and indices for gas leases by region are arranged in similar order. These appendices are: Appendix I--south Texas, Appendix J--south Louisiana, Appendix K--north Louisiana, Appendix L-- MidContinent, and Appendix M--Rocky Mountain Region.

Notes: - 1995 data are preliminary and are marked with a single asterisk $\left(^{*}\right)$. * All prior data were revised. • Indices marked with a double asterisk $\left(^{* *}\right)$ are composite indices. - Other indices are pure cost. 


\begin{tabular}{|c|c|c|c|c|c|}
\hline \multirow{2}{*}{ Component } & \multicolumn{4}{|c|}{ Index $(1976=100)$} & \multirow{2}{*}{$\begin{array}{c}1995^{*} \\
\text { Cost } \\
\text { (dollars) }\end{array}$} \\
\hline & 1992 & 1993 & 1994 & 1995 & \\
\hline & \multicolumn{5}{|c|}{50 Thousand Cubic Feet Per Day } \\
\hline Flowlines and Connections.............................. & 153.8 & 176.9 & 176.9 & 184.6 & 2,400 \\
\hline Production Package......................................... & 161.8 & 170.6 & 170.6 & 170.6 & 5,800 \\
\hline 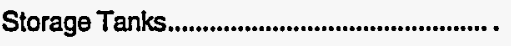 & 187.0 & 198.1 & 179.6 & 188.9 & 10,200 \\
\hline \multirow[t]{2}{*}{ 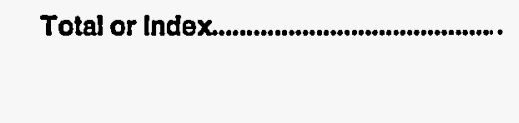 } & 174.3 & 186.1 & 176.2 & 182.2 & 18,400 \\
\hline & \multicolumn{5}{|c|}{250 Thousand Cubic Feet Per Day } \\
\hline Flowlines and Connections............................. & 153.8 & 176.9 & 176.9 & 184.6 & 2,400 \\
\hline 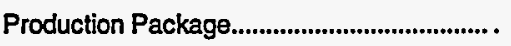 & 161.8 & 170.6 & 170.6 & 170.6 & 5,800 \\
\hline 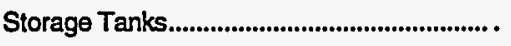 & 187.0 & 198.1 & 179.6 & 188.9 & 10,200 \\
\hline Total or Index & 174.3 & 186.1 & 176.2 & 182.2 & 18,400 \\
\hline
\end{tabular}

Table H2. Lease Equipment Costs and Indices for Gas Production in West Texas (1 Well Producing from 4,000 Feet)

\begin{tabular}{|c|c|c|c|c|c|}
\hline \multirow{3}{*}{ Component } & \multirow{2}{*}{\multicolumn{4}{|c|}{ Index $(1976=100)$}} & \multirow{3}{*}{$\begin{array}{c}\text { 1995* } \\
\text { Cost } \\
\text { (dollars) }\end{array}$} \\
\hline & & & & & \\
\hline & 1992 & 1993 & 1994 & 1995 & \\
\hline & \multicolumn{5}{|c|}{50 Thousand Cubic Feet Per Day } \\
\hline Fowlines and Connections........................... & 153.8 & 176.9 & 176.9 & 184.6 & 2,400 \\
\hline Production Package....................................... & 161.8 & 170.6 & 170.6 & 170.6 & 5,800 \\
\hline Storage Tanks.............................................. & 187.0 & 198.1 & 179.6 & 188.9 & 10,200 \\
\hline \multirow[t]{2}{*}{ Total or Index....................................... } & 174.3 & 186.1 & 176.2 & 182.2 & 18,400 \\
\hline & \multicolumn{5}{|c|}{250 Thousand Cubic Feet Per Day } \\
\hline Flowlines and Connections............................ & 208.9 & 217.8 & 186.7 & 200.0 & 9,000 \\
\hline Production Package....................................... & 171.4 & 182.9 & 182.9 & 182.9 & 6,400 \\
\hline Storage Tanks.................................................. . & 187.0 & 198.1 & 179.6 & 188.9 & 10,200 \\
\hline Total or Index............................................ . & 190.3 & 200.7 & 182.8 & 191.0 & 25,600 \\
\hline
\end{tabular}


Table H3. Lease Equipment Costs and Indices for Gas Production in West Texas (1 Well Producing from 8,000 Feet)

\begin{tabular}{|c|c|c|c|c|c|}
\hline \multirow[t]{2}{*}{ Component } & \multicolumn{4}{|c|}{ Index $(1976=100)$} & \multirow{2}{*}{$\begin{array}{c}\text { 1995" } \\
\text { Cost } \\
\text { (dollars) }\end{array}$} \\
\hline & 1992 & 1993 & 1994 & 1995 & \\
\hline & \multicolumn{5}{|c|}{50 Thousand Cuble Feet Per Day } \\
\hline Flowlines and Connections............................ & 204.2 & 210.4 & 185.4 & 197.9 & 9,500 \\
\hline Production Package........................................ . & 189.7 & 200.0 & 200.0 & 200.0 & 5,800 \\
\hline Storage Tanks ..................................................... & 187.0 & 198.1 & 179.6 & 188.9 & 10,200 \\
\hline \multirow[t]{2}{*}{ Total or Index..................................... } & 193.9 & 203.1 & 186.3 & 194.7 & 25,500 \\
\hline & \multicolumn{5}{|c|}{250 Thousand Cuble Feet Per Day } \\
\hline Flowlines and Connections............................ & 204.2 & 210.4 & 185.4 & 197.9 & 9,500 \\
\hline Production Package & 171.4 & 182.9 & 182.9 & 182.9 & 6,400 \\
\hline 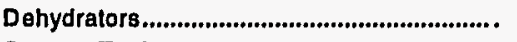 & 181.7 & 183.9 & 158.1 & 157.0 & 14,600 \\
\hline 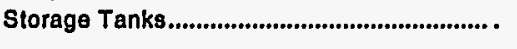 & 187.0 & 198.1 & 179.6 & 188.9 & 10,200 \\
\hline \multirow[t]{2}{*}{ Total or Index........................................... . } & 186.1 & 192.6 & 172.6 & 177.0 & 40,700 \\
\hline & \multicolumn{5}{|c|}{500 Thousand Cuble Feet Per Day } \\
\hline Flowlines and Connections.... & 226.8 & 231.7 & 202.4 & 217.1 & 8,900 \\
\hline Production Package............ & 150.0 & 160.0 & 160.0 & 160.0 & 6,400 \\
\hline Dehydrators.................... & 181.7 & 183.9 & 158.1 & 157.0 & 14,600 \\
\hline Storage Tanks................ & 187.0 & 198.1 & 179.6 & 188.9 & 10,200 \\
\hline 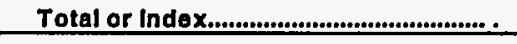 & 185.5 & 191.7 & 171.5 & 175.9 & 40,100 \\
\hline
\end{tabular}

Table H4. Lease Equipment Costs and Indices for Gas Production in West Texas (1. Well Producing from 12,000 Feet)

\begin{tabular}{|c|c|c|c|c|c|}
\hline \multirow[t]{2}{*}{ Component } & \multicolumn{4}{|c|}{ Index $(1976=100)$} & \multirow{2}{*}{$\begin{array}{c}\text { 1995* } \\
\text { Cost } \\
\text { (dollars) }\end{array}$} \\
\hline & 1992 & 1993 & 1994 & 1995 & \\
\hline & \multicolumn{5}{|c|}{250 Thousand Cubic Feet Per Day } \\
\hline Flowlines and Connections.......................... & 285.5 & 291.3 & 291.3 & $\mathbf{3 1 3 . 0}$ & 21,600 \\
\hline Production Package....................................... & 150.0 & 160.0 & 160.0 & 160.0 & 6,400 \\
\hline 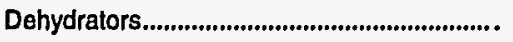 & 181.7 & 183.9 & 158.1 & 157.0 & 14,600 \\
\hline Storage Tanks.................................................... & 187.0 & 198.1 & 179.6 & 188.9 & 10,200 \\
\hline \multirow[t]{2}{*}{ Total or Index.......................................... } & 205.9 & 212.1 & 198.8 & 206.3 & 52,800 \\
\hline & \multicolumn{5}{|c|}{500 Thousand Cuble Feet Per Day } \\
\hline Flowlines and Connections........................... & 309.7 & 314.5 & 316.1 & 338.7 & 21,000 \\
\hline Production Package.......................................... . & 150.0 & 160.0 & 160.0 & 160.0 & 6,400 \\
\hline Dehydrators...................................................... & 181.7 & 183.9 & 158.1 & 157.0 & 14,600 \\
\hline Storage Tanks................................................. & 187.0 & 198.1 & 179.6 & 188.9 & 10,200 \\
\hline \multirow[t]{2}{*}{ Total or Index........................................... } & 209.6 & 215.7 & 202.4 & 209.6 & 52,200 \\
\hline & \multicolumn{4}{|c|}{1 Million Cubic Feet Per Day } & \\
\hline Flowlines and Connections............................ & 321.2 & 323.1 & 323.1 & 348.1 & 18,100 \\
\hline Production Package......................................... . & 145.5 & 150.5 & 109.1 & 109.1 & 10,800 \\
\hline 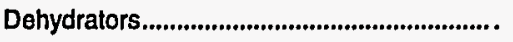 & 181.7 & 183.9 & 158.1 & 157.0 & 14,600 \\
\hline Storage Tanks................................................. & 187.0 & 198.1 & 179.6 & 188.9 & 10,200 \\
\hline Total or Indox.............................................. & 195.0 & 199.7 & 174.5 & 180.2 & 53,700 \\
\hline
\end{tabular}


Table H5. Lease Equipment Costs and Indices for Gas Production in West Texas (1 Well Producing from 16,000 Feet)

\begin{tabular}{|c|c|c|c|c|c|}
\hline \multirow[t]{2}{*}{ Component } & \multicolumn{4}{|c|}{ Index $(1976=100)$} & \multirow{2}{*}{$\begin{array}{c}\text { 1995* } \\
\text { Cost } \\
\text { (dollars) }\end{array}$} \\
\hline & 1992 & 1993 & 1994 & 1995 & \\
\hline - & \multicolumn{5}{|c|}{ 500 Thousand Cublc Feet Per Day } \\
\hline Flowlines and Connections............................ . & 321.2 & 323.1 & 323.1 & 348.1 & 18,100 \\
\hline Production Package ...................................... . & 145.5 & 150.5 & 109.1 & 109.1 & 10,800 \\
\hline 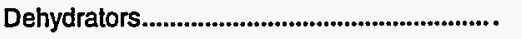 & 181.7 & 183.9 & 158.1 & 157.0 & 14,600 \\
\hline 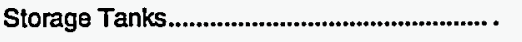 & 187.0 & 198.1 & 179.6 & 188.9 & 10,200 \\
\hline \multirow[t]{2}{*}{ Total or Index........................................... . } & 195.0 & 199.7 & 174.5 & 180.2 & 53,700 \\
\hline & \multicolumn{5}{|c|}{1 Million Cuble Feet Per Day } \\
\hline Flowlines and Connections.............................. & 321.2 & 323.1 & 323.1 & 348.1 & 18,100 \\
\hline Production Package...................................... & 145.5 & 150.5 & 109.1 & 109.1 & 10,800 \\
\hline 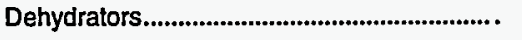 & 181.7 & 183.9 & 158.1 & 157.0 & 14,600 \\
\hline 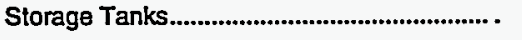 & 187.0 & 198.1 & 179.6 & 188.9 & 10,200 \\
\hline \multirow[t]{2}{*}{ 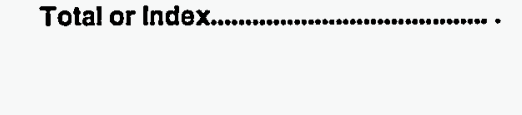 } & 195.0 & 199.7 & 174.5 & 180.2 & 53,700 \\
\hline & \multicolumn{4}{|c|}{5 Million Cubic Feot Per Day } & \\
\hline Flowlines and Connections.............................. & 303.7 & 304.3 & 301.2 & 323.8 & 53,100 \\
\hline Production Package.......................................... & 145.4 & 151.9 & 129.6 & 123.1 & 13,300 \\
\hline 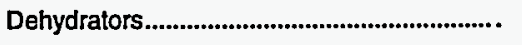 & 203.5 & 207.1 & 187.6 & 187.6 & 21,200 \\
\hline 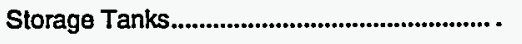 & 187.0 & 198.1 & 179.6 & 188.9 & 10,200 \\
\hline Total or Index............................................. . & 224.6 & 228.7 & 214.8 & 222.8 & 97,800 \\
\hline
\end{tabular}

Table H6. Direct Annual Operating Costs and Indices for Gas Production in West Texas (1 Well Producing from 2,000 Feet)

\begin{tabular}{|c|c|c|c|c|c|}
\hline \multirow[t]{2}{*}{ Component } & \multicolumn{4}{|c|}{ Index $(1976=100)$} & \multirow{2}{*}{$\begin{array}{c}\text { 1995* } \\
\text { Cost } \\
\text { (dollars) }\end{array}$} \\
\hline & 1992 & 1993 & 1994 & 1995 & \\
\hline & \multicolumn{5}{|c|}{50 Thousand Cubic Feet Per Day } \\
\hline Direct Labor \& Overhead................................ . & 275.0 & 275.0 & 283.3 & 283.3 & 3,400 \\
\hline Fuel, Chemicals \& Disposal........................... & 300.0 & 300.0 & 300.0 & 300.0 & 600 \\
\hline Surface Maintenance......................................... & 240.0 & 233.3 & 233.3 & 233.3 & 3,500 \\
\hline Subsurface Maintenance................................. & 180.0 & 180.0 & 180.0 & $180.0^{\circ}$ & 900 \\
\hline \multirow[t]{2}{*}{ Total or Index $x^{* \star} \ldots \ldots \ldots \ldots \ldots \ldots$} & 247.1 & 244.1 & 247.1 & 247.1 & 8,400 \\
\hline & \multicolumn{5}{|c|}{ 250 Thousand Cubic Feet Per Day } \\
\hline Direct Labor \& Overhead.................................. . & 275.0 & 275.0 & 283.3 & 283.3 & 3,400 \\
\hline Fuel, Chemicals \& Disposal........................... & 220.0 & 220.0 & 220.0 & 220.0 & 2,200 \\
\hline Surface Maintenance........................................ & 240.0 & 233.3 & 233.3 & 233.3 & 3,500 \\
\hline Subsurface Maintenance..................................... & 180.0 & 180.0 & 180.0 & 180.0 & 900 \\
\hline Total or Index ${ }^{* *}$ & 238.1 & 235.7 & 238.1 & 238.1 & 10,000 \\
\hline
\end{tabular}


Table H7. Direct Annual Operating Costs and Indices for Gas Production in West Texas (1 Well Producing from 4,000 Feet)

\begin{tabular}{|c|c|c|c|c|c|}
\hline \multirow[t]{2}{*}{ Component } & \multicolumn{4}{|c|}{ Index $(1976=100)$} & \multirow{2}{*}{$\begin{array}{c}\text { 1995* } \\
\text { Cost } \\
\text { (dollars) }\end{array}$} \\
\hline & 1992 & 1993 & 1994 & 1995 & \\
\hline & \multicolumn{5}{|c|}{50 Thousand Cubic Feet Per Day } \\
\hline Direct Labor \& Overhead................................... . & 264.3 & 271.4 & 271.4 & 271.4 & 3,800 \\
\hline Fuel, Chemicals \& Disposal............................ & 220.0 & 220.0 & 220.0 & 220.0 & 1,100 \\
\hline Surface Maintenance..................................... & 266.7 & 260.0 & 260.0 & 260.0 & 3,900 \\
\hline Subsurface Maintenance................................... & 185.7 & 185.7 & 185.7 & 185.7 & 1,300 \\
\hline \multirow[t]{2}{*}{ Total or Index* *........................................... } & 246.3 & 246.3 & 246.3 & 246.3 & 10,100 \\
\hline & \multicolumn{5}{|c|}{250 Thousand Cubic Feet Per Day } \\
\hline Direct Labor \& Overhead.................................... . & 264.3 & 271.4 & 271.4 & 271.4 & 3,800 \\
\hline Fuel, Chemicals \& Disposal............................. & 220.0 & 220.0 & 220.0 & 220.0 & 4,400 \\
\hline Surface Maintenance...................................... & 230.0 & 225.0 & 220.0 & 220.0 & 4,400 \\
\hline Subsurface Maintenance................................. & 185.7 & 185.7 & 185.7 & 185.7 & 1,300 \\
\hline Total or Index & 229.5 & 229.5 & 227.9 & 227.9 & 13,900 \\
\hline
\end{tabular}

Table HB. Direct Annual Operating Costs and Indices for Gas Production in West Texas (1 Well Producing from 8,000 Feet)

\begin{tabular}{|c|c|c|c|c|c|}
\hline \multirow[t]{2}{*}{ Component } & \multicolumn{4}{|c|}{ Index $(1976=100)$} & \multirow{2}{*}{$\begin{array}{c}1995^{*} \\
\text { Cost } \\
\text { (dollars) }\end{array}$} \\
\hline & 1992 & 1993 & 1994 & 1995 & \\
\hline & \multicolumn{5}{|c|}{50 Thousand Cuble Feet Per Day } \\
\hline Direct Labor \& Overhead................................. . & 268.8 & 275.0 & 275.0 & 281.3 & 4,500 \\
\hline Fuel, Chemicals \& Disposal.......................... & 220.0 & 220.0 & 220.0 & 220.0 & 2,200 \\
\hline Surface Maintenance...................................... & 230.0 & 225.0 & 220.0 & 220.0 & 4,400 \\
\hline Subsurface Maintenance................................ & 175.0 & 175.0 & 175.0 & 175.0 & 2,100 \\
\hline \multirow[t]{2}{*}{ Total or Index $x^{* * \ldots \ldots \ldots \ldots \ldots \ldots \ldots \ldots \ldots \ldots \ldots \ldots}$} & 227.6 & 227.6 & 225.9 & 227.6 & 13,200 \\
\hline & \multicolumn{5}{|c|}{250 Thousand Cuble Feet Per Day } \\
\hline Direct Labor \& Overhead................................. . & 268.8 & 275.0 & 275.0 & 281.3 & 4,500 \\
\hline Fuel, Chemicals \& Disposal........................... & 228.2 & 228.2 & 228.2 & 228.2 & 8,900 \\
\hline Surface Maintenance...................................... & 228.2 & 223.1 & 217.9 & 217.9 & 8,500 \\
\hline Subsurface Maintenance................................... & 175.0 & 175.0 & 175.0 & 175.0 & 2,100 \\
\hline \multirow[t]{2}{*}{ Total or Index ${ }^{*}$} & 228.3 & 227.4 & 225.5 & 226.4 & 24,000 \\
\hline & \multicolumn{5}{|c|}{500 Thousand Cuble Feet Per Day } \\
\hline Direct Labor \& Overhead.................................. . & 268.8 & 275.0 & 275.0 & 281.3 & 4,500 \\
\hline Fuel, Chemicals \& Disposal......................... & 238.1 & 238.1 & 238.1 & 233.3 & 4,900 \\
\hline Surface Malntenance................................... & 229.4 & 223.5 & 217.6 & 217.6 & 7,400 \\
\hline Subsurface Malntenance............................... & 175.0 & 175.0 & 175.0 & 175.0 & 2,100 \\
\hline 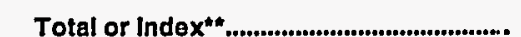 & 231.3 & 230.1 & 227.7 & 227.7 & 18,900 \\
\hline
\end{tabular}


Table H9. Direct Annual Operating Costs and Indlces for Gas Production In West Texas (1 Well Producing from 12,000 Feet)

\begin{tabular}{|c|c|c|c|c|c|}
\hline \multirow[t]{2}{*}{ Component } & \multicolumn{4}{|c|}{ Index $(1976=100)$} & \multirow{2}{*}{$\begin{array}{c}\text { 1995* } \\
\text { Cost } \\
\text { (dollars) }\end{array}$} \\
\hline & 1992 & 1993 & 1994 & 1995 & \\
\hline & \multicolumn{5}{|c|}{250 Thousand Cuble Feet Per Day } \\
\hline Direct Labor \& Overhead...................................... . & 273.7 & 278.9 & 278.9 & 284.2 & 5,400 \\
\hline Fuel, Chemicals \& Disposal............................. & 222.0 & 222.0 & 222.0 & 222.0 & 11,100 \\
\hline Surface Maintenance..................................... & 222.5 & 217.5 & 212.5 & 212.5 & 8,500 \\
\hline Subsurface Maintenance.................................... & 188.5 & 188.5 & 188.5 & 188.5 & 4,900 \\
\hline \multirow[t]{2}{*}{ Total or Index } & 223.0 & 222.2 & 220.7 & 221.5 & 29,900 \\
\hline & \multicolumn{5}{|c|}{500 Thousand Cuble Feet Per Day } \\
\hline Direct Labor \& Overhead................................... . & 273.7 & 278.9 & 278.9 & 284.2 & 5,400 \\
\hline Fuel, Chemicals \& Disposal............................ & 224.0 & 224.0 & 220.0 & 220.0 & 5,500 \\
\hline Surface Maintenance...................................... & 222.9 & 217.1 & 211.4 & 211.4 & 7,400 \\
\hline 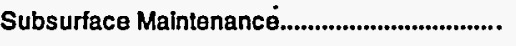 & 188.5 & 188.5 & 188.5 & 188.5 & 4,900 \\
\hline \multirow[t]{2}{*}{ 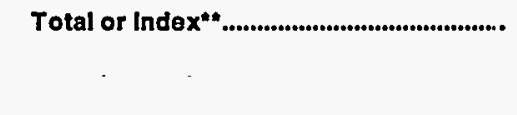 } & 223.8 & 222.9 & 220.0 & 221.0 & 23,200 \\
\hline & \multicolumn{5}{|c|}{1 Million Cubic Feet Per Day } \\
\hline Direct Labor \& Overhead................................ . & 273.7 & 278.9 & 278.9 & 284.2 & 5,400 \\
\hline Fuel, Chemicals \& Disposal........................... & 226.9 & 226.9 & 225.0 & 223.1 & 11,600 \\
\hline Surface Maintenance......................................... & 212.8 & 207.7 & 200.0 & 200.0 & 7,800 \\
\hline 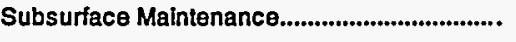 & 188.5 & 188.5 & 188.5 & 188.5 & 4,900 \\
\hline Total or Index & 222.1 & 221.3 & 218.4 & 218.4 & 29,700 \\
\hline
\end{tabular}

Table H10. Direct Annual Operating Costs and Indices for Gas Production In West Texas (1 Woll Producing from 16,000 Foot)

\begin{tabular}{|c|c|c|c|c|c|}
\hline \multirow[t]{2}{*}{ Component } & \multicolumn{4}{|c|}{ Index $(1976=100)$} & \multirow{2}{*}{$\begin{array}{c}\text { 1995* } \\
\text { Cost } \\
\text { (dollars) }\end{array}$} \\
\hline & 1992 & 1993 & 1994 & 1995 & \\
\hline & \multicolumn{5}{|c|}{500 Thousand Cubic Feot Per Day } \\
\hline Direct Labor \& Overhead.................................. . & 273.7 & 278.9 & 278.9 & 284.2 & 5,400 \\
\hline Fuel, Chemicals \& Disposal.......................... & 222.6 & 222.6 & 222.6 & 219.4 & 6,800 \\
\hline Surface Malntenance........................................ & 225.6 & 223.1 & 215.4 & 217.9 & 8,500 \\
\hline Subsurface Maintenance................................ & 183.9 & 183.9 & 183.9 & 183.9 & 5,700 \\
\hline \multirow[t]{2}{*}{ Total or Index } & 221.7 & 221.7 & 219.2 & 220.0 & 26,400 \\
\hline & \multicolumn{5}{|c|}{1 Million Cubic Feet For Day } \\
\hline Direct Labor \& Overhead................................ . & 273.7 & 278.9 & 278.9 & 284.2 & 5,400 \\
\hline Fuel, Chemicals \& Dlsposal........................... & 223.0 & 221.3 & 219.7 & 219.7 & 13,400 \\
\hline Surface Maintenance......................................... & 225.6 & 223.1 & 215.4 & 217.9 & 8,500 \\
\hline Śubsurface Maintenance................................... & 183.9 & 183.9 & 183.9 & 183.9 & 5,700 \\
\hline \multirow[t]{2}{*}{ Total or Index } & 222.0 & 221.3 & 218.7 & 220.0 & 33,000 \\
\hline & \multicolumn{5}{|c|}{5 Million Cubic Feet Per Day } \\
\hline Direct Labor \& Overhead.................................. . & 273.7 & 278.9 & 278.9 & 284.2 & 5,400 \\
\hline Fuel, Chemicals \& Disposal........................... & 146.5 & 153.5 & 153.5 & 153.5 & 15,200 \\
\hline Surface Maintenance...................................... & 238.8 & 236.7 & 230.6 & 234.7 & 11,500 \\
\hline Subsurface Maintenance................................... & 133.3 & 133.3 & 130.3 & 133.3 & 4,400 \\
\hline Total or Index & 179.0 & 182.5 & 180.5 & 182.5 & 36,500 \\
\hline
\end{tabular}


Table H11. Detailed Lease Equipment List for 12,000-Foot Gas Wells in West Texas Producing 1 Million Cubic Feet per Day

\section{Safety Valve}

Size: 2 inches

Working Pressure: 10,000 pounds per square inch

Actuates: High/low pressures

\section{Production Package}

Choke: Built in, inlet

Coils: 2 inch $\mathrm{XH}$

Heater rating: $250,000 \mathrm{BTU}$ per hour

Size: 16 inches by 8 feet

Working pressure: 1,000 pounds per square inch
Dehydrator/Reconcentrator

Type: Glycol absorption

Size: $12-3 / 4$ inches

Working pressure: 1,440 pounds per square inch

Storage Tanks (2)

Size: 10 feet by 15 feet

Capacity 210 barrels

Construction: Welded steel

Source: Energy Information Administration, Office of Oil and Gas 
Table I1. Lease Equipment Costs and Indices for Gas Production in South Texas (1 Well Producing from 2,000 Feet)

\begin{tabular}{|c|c|c|c|c|c|}
\hline \multirow{2}{*}{ Component } & \multicolumn{4}{|c|}{ Index $(1976=100)$} & \multirow{2}{*}{$\begin{array}{c}\text { 1995" } \\
\text { Cost } \\
\text { (dollar8) }\end{array}$} \\
\hline & 1992 & 1993 & 1994 & 1995 & \\
\hline & \multicolumn{5}{|c|}{50 Thousand Cuble Feet Per Day } \\
\hline Flowlines and Connections.............................. . & 161.5 & 184.6 & 184.6 & 192.3 & 2,500 \\
\hline Production Package............................................ & 148.6 & 154.3 & 160.0 & 160.0 & 5,600 \\
\hline 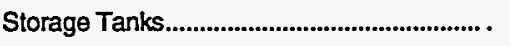 & 183.6 & 194.5 & 176.4 & 185.5 & 10,200 \\
\hline \multirow[t]{2}{*}{ 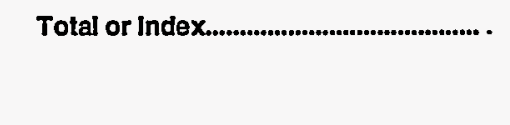 } & 168.9 & 179.6 & 171.8 & 177.7 & 18,300 \\
\hline & \multicolumn{5}{|c|}{ 250 Thousand Cubic Feet Per Day } \\
\hline Flowlines and Connections............................... . & 161.5 & 184.6 & 184.6 & 192.3 & 2,500 \\
\hline Production Package.............................................. & 148.6 & 154.3 & 160.0 & 160.0 & 5,600 \\
\hline 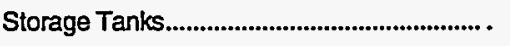 & 183.6 & 194.5 & 176.4 & 185.5 & 10,200 \\
\hline 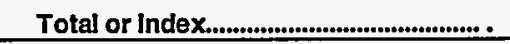 & 168.9 & 179.6 & 171.8 & 177.7 & 18,300 \\
\hline
\end{tabular}

Table 12. Lease Equipment Costs and Indices for Gas Production in South Texas (1 Well Producing from 4,000 Feet)

\begin{tabular}{|c|c|c|c|c|c|}
\hline \multirow[t]{2}{*}{ Component } & \multicolumn{4}{|c|}{ Index $(1976=100)$} & \multirow{2}{*}{$\begin{array}{c}\text { 1995" } \\
\text { Cost } \\
\text { (dollars) }\end{array}$} \\
\hline & 1992 & 1993 & 1994 & 1995 & \\
\hline & \multicolumn{5}{|c|}{50 Thousand Cubic Feet Per Day } \\
\hline Flowlines and Connections............................ . & 161.5 & 184.6 & 184.6 & 192.3 & 2,500 \\
\hline Production Package....................................... & 148.6 & 154.3 & 160.0 & 160.0 & 5,600 \\
\hline Storage Tanks............................................... & 183.6 & 194.5 & 176.4 & 185.5 & 10,200 \\
\hline \multirow[t]{2}{*}{ Total or Index......................................... } & 168.9 & 179.6 & 171.8 & 177.7 & 18,300 \\
\hline & \multicolumn{5}{|c|}{250 Thousand Cuble Feet Per Day } \\
\hline Flowlines and Connections............................ . & 206.5 & 213.0 & 184.8 & 195.7 & 9,000 \\
\hline Production Package......................................... . & 154.1 & 162.2 & 170.3 & 170.3 & 6,300 \\
\hline 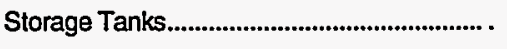 & 183.6 & 194.5 & 176.4 & 185.5 & 10,200 \\
\hline Total or Index......................................... . & 183.3 & 192.0 & 177.5 & 184.8 & 25,500 \\
\hline
\end{tabular}




\begin{tabular}{|c|c|c|c|c|c|}
\hline \multirow[t]{2}{*}{ Component } & \multicolumn{4}{|c|}{ Index $(1976=100)$} & \multirow{2}{*}{$\begin{array}{c}\text { 1995* } \\
\text { Cost } \\
\text { (dollars) }\end{array}$} \\
\hline & 1992 & 1993 & 1994 & 1995 & \\
\hline & \multicolumn{5}{|c|}{$\cdot$} \\
\hline Flowlines and Connections................................. & 200.0 & 206.1 & 183.7 & 195.9 & 9,600 \\
\hline Production Package.............................................. & 154.1 & 162.2 & 170.3 & 170.3 & 6,300 \\
\hline 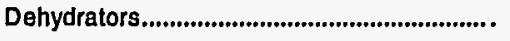 & 176.8 & 183.2 & 157.9 & 156.8 & 14,900 \\
\hline Storage Tanks........................................................ & 183.6 & 194.5 & 176.4 & 185.5 & 10,200 \\
\hline \multirow[t]{2}{*}{ 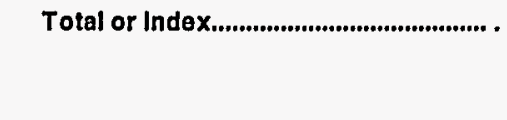 } & 179.7 & 187.3 & 169.5 & 173.7 & 41,000 \\
\hline & \multicolumn{5}{|c|}{500 Thousand Cubic Feet Per Day } \\
\hline Flowlines and Connections................................ & 226.8 & 231.7 & 202.4 & 217.1 & 8,900 \\
\hline 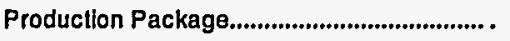 & 135.7 & 142.9 & 150.0 & 150.0 & 6,300 \\
\hline 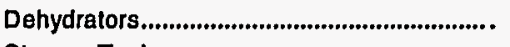 & 176.8 & 183.2 & 157.9 & 156.8 & 14,900 \\
\hline 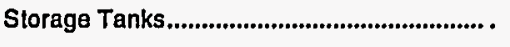 & 183.6 & 194.5 & 176.4 & 185.5 & 10,200 \\
\hline \multirow[t]{2}{*}{ 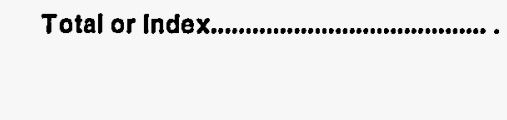 } & 179.8 & 187.1 & 168.7 & 173.0 & 40,300 \\
\hline & \multicolumn{5}{|c|}{1 Million Cubic Feet Per Day } \\
\hline Flowlines and Connections............................. & 321.2 & 323.1 & 323.1 & 348.1 & 18,100 \\
\hline 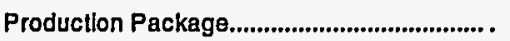 & 146.0 & 150.0 & 109.0 & 109.0 & 10,900 \\
\hline 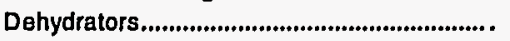 & 176.8 & 183.2 & 157.9 & 156.8 & 14,900 \\
\hline Storage Tanks......................................................... & 187.0 & 198.1 & 179.6 & 188.9 & 10,200 \\
\hline 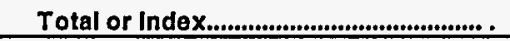 & 193.4 & 199.0 & 174.1 & 179.7 & 54,100 \\
\hline
\end{tabular}

Table 14. Lease Equipment Costs and Indices for Gas Production in South Texas (1 Well Producing from 12,000 Feet)

\begin{tabular}{|c|c|c|c|c|c|}
\hline \multirow[t]{2}{*}{ Component } & \multicolumn{4}{|c|}{ Index $(1976=100)$} & \multirow{2}{*}{$\begin{array}{c}1995^{\circ} \\
\text { Cost } \\
\text { (dollars) }\end{array}$} \\
\hline & 1992 & 1993 & 1994 & 1995 & \\
\hline & \multicolumn{5}{|c|}{500 Thousand Cubic Feet Per Day } \\
\hline Flowlines and Connections............................ & 309.7 & 314.5 & 316.1 & 338.7 & 21,000 \\
\hline Production Package.......................................... & 135.7 & 142.9 & 150.0 & 150.0 & 6,300 \\
\hline 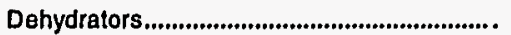 & 176.8 & 183.2 & 157.9 & 156.8 & 14,900 \\
\hline 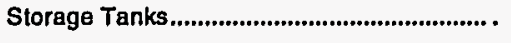 & 183.6 & 194.5 & 176.4 & 185.5 & 10,200 \\
\hline \multirow[t]{2}{*}{ 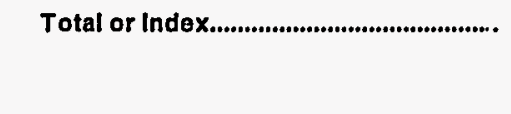 } & 203.9 & 211.0 & 199.2 & 206.3 & 52,400 \\
\hline & \multicolumn{5}{|c|}{1 Million Cubic Feet Per Day } \\
\hline Flowlines and Connections............................. & 321.2 & 323.1 & 323.1 & 348.1 & 18,100 \\
\hline Production Package........................................... & 146.0 & 150.0 & 109.0 & 109.0 & 10,900 \\
\hline 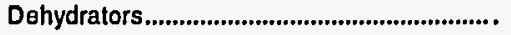 & 176.8 & 183.2 & 157.9 & 156.8 & 14,900 \\
\hline 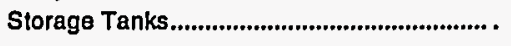 & 183.6 & 194.5 & 176.4 & .185 .5 & 10,200 \\
\hline \multirow[t]{2}{*}{ 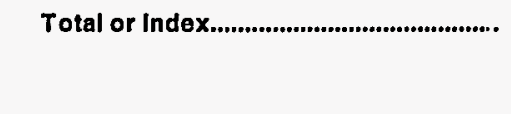 } & 192.7 & 198.3 & 173.5 & 179.1 & 54,100 \\
\hline & \multicolumn{5}{|c|}{5 Million Cubic Feet Per Day } \\
\hline Flowlines and Connections.............................. & 303.7 & 304.3 & 301.2 & 323.8 & 53,100 \\
\hline Production Package........................................... & 145.5 & 150.9 & 129.1 & 123.6 & 13,600 \\
\hline 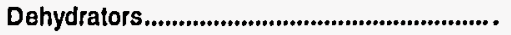 & 200.0 & 206.1 & 187.0 & 187.0 & 21,500 \\
\hline 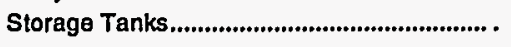 & 183.6 & 194.5 & 176.4 & 185.5 & 10,200 \\
\hline 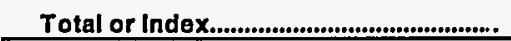 & 222.7 & 227.3 & 213.5 & 221.6 & 98,400 \\
\hline
\end{tabular}


Table 15. Direct Annual Operating Costs and Indices for Gas Production in South Texas (1 Well Producing from 2,000 Feet)

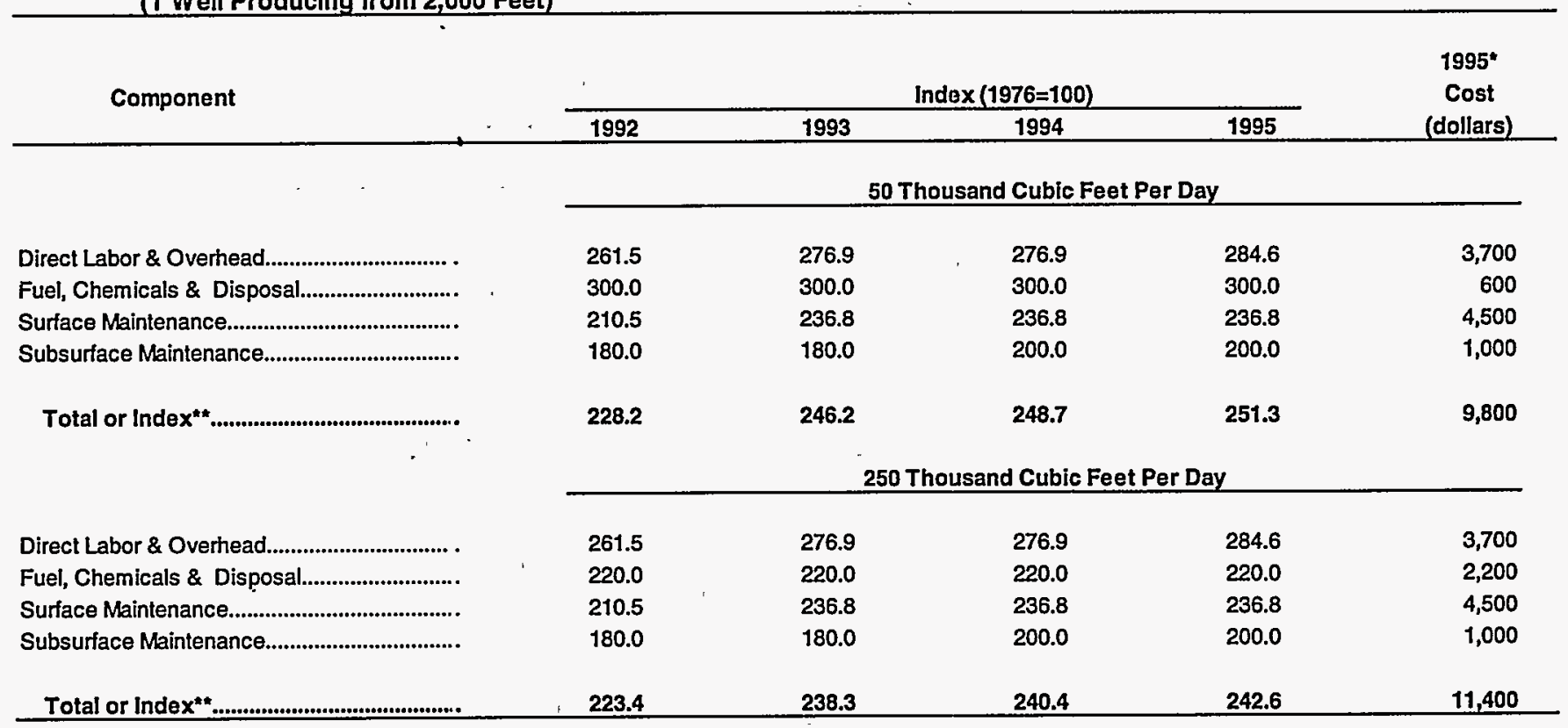

Table 16. Direct Annual Operating Costs and Indices for Gas Production in South Texas (1 Well Producing from 4,000 Feet)

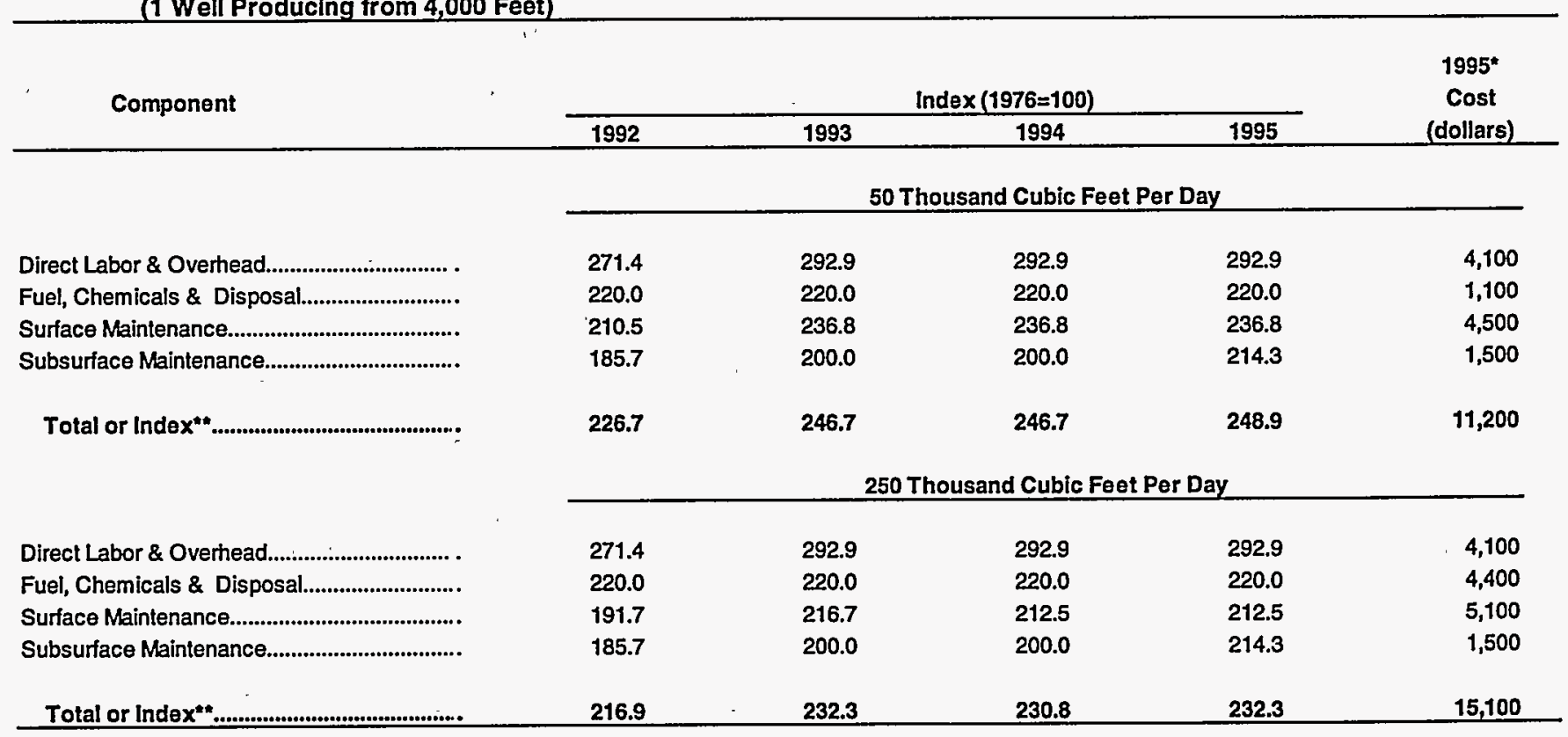


Table 17. Direct Annual Operating Costs and Indices for Gas Production in South Texas (1 Well Producing from 8,000 Feot)

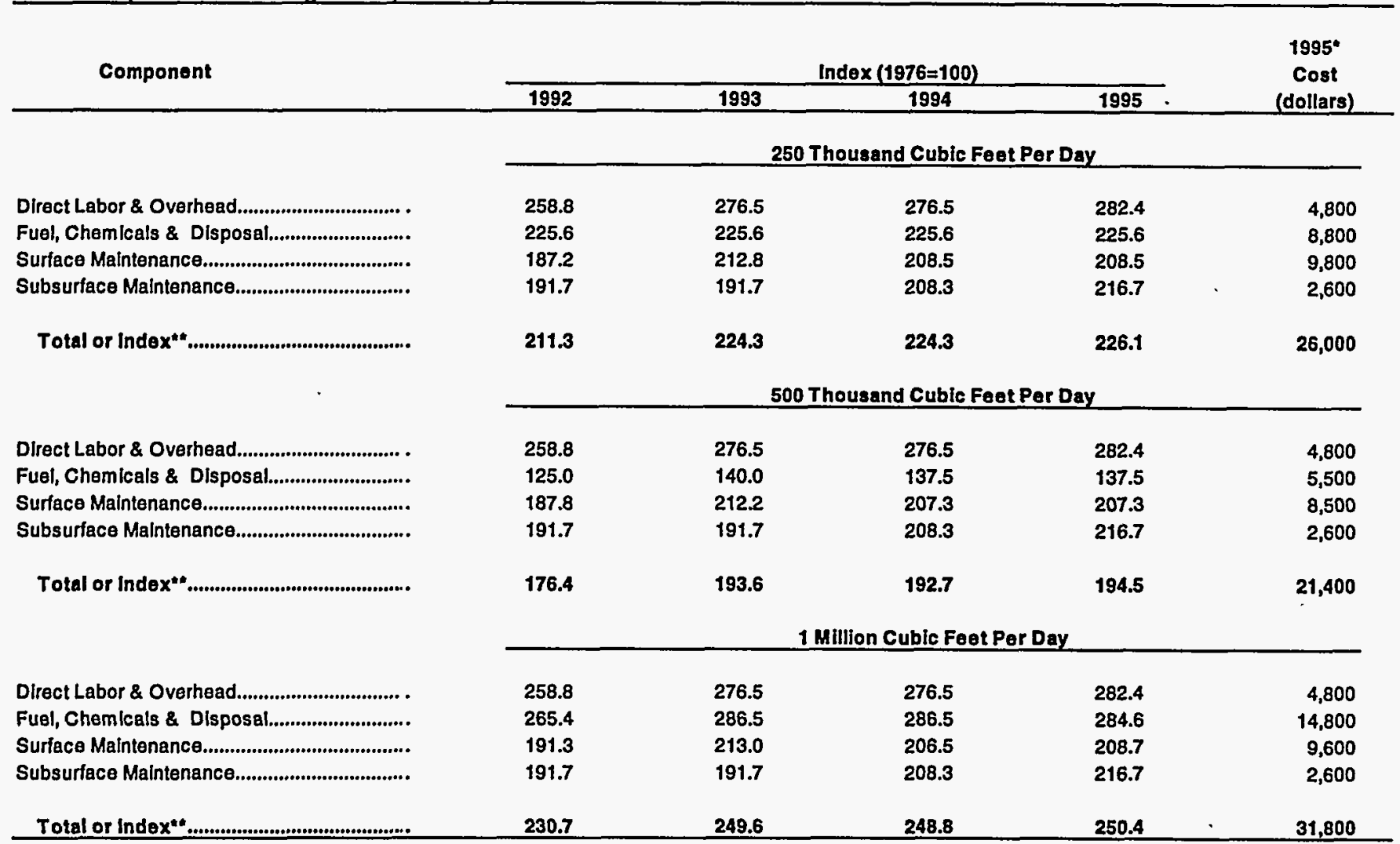

Table 18. Direct Annual Operating Costs and Indices for Gas Production in South Texas (1 Well Producing from 12,000 Feet)

\begin{tabular}{|c|c|c|c|c|c|}
\hline \multirow[t]{2}{*}{ Component } & \multicolumn{4}{|c|}{ index $(1976=100)$} & \multirow{2}{*}{$\begin{array}{c}\text { 1995 } \\
\text { Cost } \\
\text { (dollars) }\end{array}$} \\
\hline & 1992 & 1993 & 1994 & 1995 & \\
\hline & \multicolumn{5}{|c|}{ 500 Thousand Cublc Foet Per Day } \\
\hline Direct Labor \& Overhead................................ . & 265.0 & 285.0 & 285.0 & 290.0 & 5,800 \\
\hline Fuel, Chemicals \& Disposal........................... & 196.6 & 217.2 & 217.2 & 213.8 & 6,200 \\
\hline Surface Malntenance.................................... & 197.6 & 219.0 & 216.7 & 219.0 & 9,200 \\
\hline Subsurface Maintenance................................... & 200.0 & 204.2 & 216.7 & 225.0 & 5,400 \\
\hline \multirow[t]{2}{*}{ Total or Index } & 209.6 & 227.0 & 228.7 & 231.3 & 26,600 \\
\hline & \multicolumn{5}{|c|}{1 Million Cubic Foot Por Day } \\
\hline Direct Labor \& Overhead................................. . & 265.0 & 285.0 & 285.0 & 290.0 & 5,800 \\
\hline Fuel, Chemicals \& Disposal.......................... & 164.4 & 183.1 & 181.4 & 181.4 & 10,700 \\
\hline Surface Malntenance.................................... & 191.3 & 213.0 & 206.5 & 208.7 & 9,600 \\
\hline Subsurface Maintenance................................. & 200.0 & 204.2 & 216.7 & 225.0 & 5,400 \\
\hline \multirow[t]{2}{*}{ Total or Index } & 191.9 & 209.4 & 208.7 & 211.4 & 31,500 \\
\hline & \multicolumn{5}{|c|}{5 Million Cuble Feot Por Day } \\
\hline Direct Labor \& Overhead................................. . & 265.0 & 285.0 & 285.0 & 290.0 & 5,800 \\
\hline Fual, Chemicals \& Disposal.......................... & 156.1 & 164.9 & 163.2 & 163.2 & 9,300 \\
\hline Surface Maintenance..................................... & 185.7 & 203.2 & 198.4 & 201.6 & 12,700 \\
\hline Subsurface Malntenance................................. & 184.6 & 188.5 & 200.0 & 207.7 & 5,400 \\
\hline Total or Index & 184.9 & 197.6 & 197.0 & 200.0 & 33,200 \\
\hline
\end{tabular}


Table J1. Lease Equipment Costs and Indices for Gas Production in South Louisiana (1 Well Producing from 2,000 Feet)

\begin{tabular}{|c|c|c|c|c|c|}
\hline \multirow[t]{2}{*}{ Component } & \multicolumn{4}{|c|}{ Index $(1976=100)$} & \multirow{2}{*}{$\begin{array}{c}1995^{*} \\
\text { Cost } \\
\text { (dollar8) }\end{array}$} \\
\hline & 1992 & 1993 & 1994 & 1995 & \\
\hline- & \multicolumn{5}{|c|}{50 Thougand Cubic Feet Per Day } \\
\hline Flowlines and Connections............................ & 150.0 & 164.3 & 164.3 & 178.6 & 2,500 \\
\hline Production Package......................................... & 148.6 & 151.4 & 160.0 & 162.9 & 5,700 \\
\hline 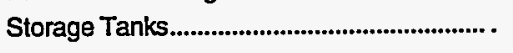 & 183.9 & 191.1 & 175.0 & 183.9 & 10,300 \\
\hline Total or Index......................................... & 167.6 & 174.3 & 168.6 & 176.2 & 18,500 \\
\hline- & \multicolumn{4}{|c|}{ 250 Thousand Cubic Foot Per Day } & \\
\hline Flowlines and Connections............................ . & 150.0 & 164.3 & 164.3 & 178.6 & 2,500 \\
\hline Production Package......................................... . & 148.6 & 151.4 & 160.0 & 162.9 & 5,700 \\
\hline 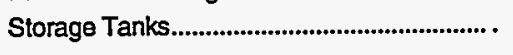 & 183.9 & 191.1 & 175.0 & 183.9 & 10,300 \\
\hline Total or Index.......................................... & 167.6 & 174.3 & 168.6 & 176.2 & 18,500 \\
\hline
\end{tabular}

Table J2. Lease Equipment Costs and Indices for Gas Production in South Louisiana (1 Well Producing from 4,000 Feet)

\begin{tabular}{|c|c|c|c|c|c|}
\hline \multirow{2}{*}{ Component } & \multicolumn{4}{|c|}{ Index $(1976=100)$} & \multirow{2}{*}{$\begin{array}{c}1995^{\star} \\
\text { Cost } \\
\text { (dollars) }\end{array}$} \\
\hline & 1992 & 1993 & 1994 & 1995 & \\
\hline & \multicolumn{5}{|c|}{50 Thousand Cubic Feet Per Day } \\
\hline Flowlines and Connections............................ . & 150.0 & 164.3 & 164.3 & 178.6 & 2,500 \\
\hline 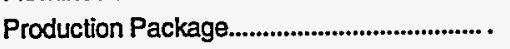 & 148.6 & $151.4^{-}$ & 160.0 & 162.9 & 5,700 \\
\hline 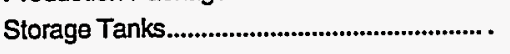 & 183.9 & 191.1 & 175.0 & 183.9 & 10,300 \\
\hline \multirow[t]{2}{*}{ 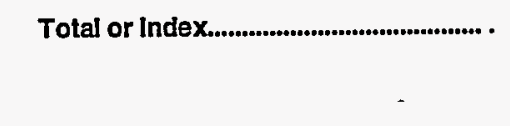 } & 167.6 & 174.3 & 168.6 & 176.2 & 18,500 \\
\hline & \multicolumn{4}{|c|}{250 Thousand Cuble Feet Per Day } & \\
\hline Flowlines and Connections............................... & 208.7 & 213.0 & 184.8 & 195.7 & 9,000 \\
\hline Production Package............................................... & 156.8 & 162.2 & 167.6 & 170.3 & 6,300 \\
\hline 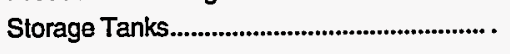 & 183.9 & 191.1 & 175.0 & 183.9 & 10,300 \\
\hline Total or Index & 184.9 & 190.6 & 176.3 & 184.2 & 25,600 \\
\hline
\end{tabular}


Table J3. Lease Equipment Costs and Indices for Gas Production in South Louisiana ( 1 Well Producing from 8,000 Feet)

\begin{tabular}{|c|c|c|c|c|c|}
\hline \multirow[t]{2}{*}{ Component } & \multicolumn{4}{|c|}{ Index $(1976=100)$} & \multirow{2}{*}{$\begin{array}{c}1995^{\star} \\
\text { Cost } \\
\text { (dollars) }\end{array}$} \\
\hline & 1992 & 1993 & 1994 & 1995 & \\
\hline & \multicolumn{5}{|c|}{250 Thousand Cubic Feet Per Day } \\
\hline$r$ & & & & & \\
\hline Flowlines and Connections.............................. & 202.0 & 206.1 & 183.7 & 195.9 & 9,600 \\
\hline Production Package.......................................... & 156.8 & 162.2 & 167.6 & 170.3 & 6,300 \\
\hline 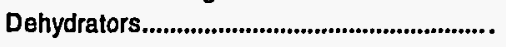 & 176.0 & 180.2 & 156.3 & 155.2 & 14,900 \\
\hline 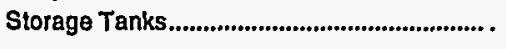 & 183.9 & 191.1 & 175.0 & 183.9 & 10,300 \\
\hline \multirow[t]{2}{*}{ 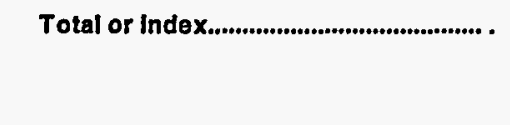 } & 180.3 & 185.3 & 168.1 & 172.7 & 41,100 \\
\hline & \multicolumn{5}{|c|}{500 Thousand Cubic Feet Per Day } \\
\hline Flowlines and Connections............................. . & 226.8 & 231.7 & 202.4 & 217.1 & 8,900 \\
\hline Production Package........................................... & 138.1 & 142.9 & 147.6 & 150.0 & 6,300 \\
\hline 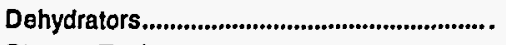 & 176.0 & 180.2 & 156.3 & 155.2 & 14,900 \\
\hline Storage Tanks ..................................................... & 183.9 & 191.1 & 175.0 & 183.9 & 10,300 \\
\hline \multirow[t]{2}{*}{ Total or Index } & 180.0 & 185.1 & 167.2 & 171.9 & 40,400 \\
\hline & \multicolumn{5}{|c|}{1 Million Cubic Feet Per Day } \\
\hline Flowlines and Connections.............................. & 321.2 & 323.1 & 323.1 & 348.1 & 18,100 \\
\hline Production Package........................................ & 146.0 & 149.0 & 109.0 & 109.0 & 10,900 \\
\hline Dehydrators........................................................ & 176.0 & 180.2 & 156.3 & 155.2 & 14,900 \\
\hline Storage Tanks.............................................. & 183.9 & 191.1 & 175.0 & 183.9 & 10,300 \\
\hline 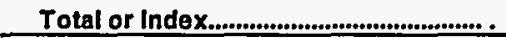 & 192.4 & 196.4 & 172.7 & 178.3 & 54,200 \\
\hline
\end{tabular}

Table J4. Lease Equipment Costs and Indlces for Gas Production in South Louislana (1 Well Producing from 12,000 Feet)

\begin{tabular}{|c|c|c|c|c|c|}
\hline \multirow[t]{2}{*}{ Component } & \multicolumn{4}{|c|}{ Index $(1976=100)$} & \multirow{2}{*}{$\begin{array}{c}1995^{\circ} \\
\text { Cost } \\
\text { (dollars) }\end{array}$} \\
\hline & 1992 & 1993 & 1994 & 1995 & \\
\hline & \multicolumn{5}{|c|}{500 Thousand Cubic Feet Per Day } \\
\hline Flowlines and Connections........................... . & 309.7 & 314.5 & 316.1 & 338.7 & 21,000 \\
\hline Production Package........................................ . & 138.1 & 142.9 & 147.6 & 150.0 & 6,300 \\
\hline 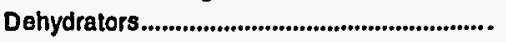 & 176.0 & 180.2 & 156.3 & 155.2 & 14,900 \\
\hline 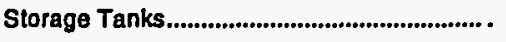 & 183.9 & 191.1 & 175.0 & 183.9 & 10,300 \\
\hline \multirow[t]{2}{*}{ 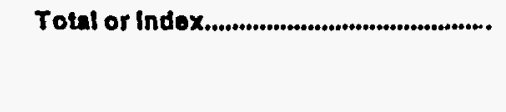 } & 203.9 & 209.0 & 197.7 & 205.1 & 52,500 \\
\hline & \multicolumn{5}{|c|}{1 Million Cuble Feat Per Day } \\
\hline Flowlines and Connections............................. & 321.2 & 323.1 & 323.1 & 348.1 & 18,100 \\
\hline Production Package......................................... . & 146.0 & 149.0 & 109.0 & 109.0 & 10,900 \\
\hline 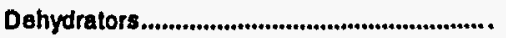 & 176.0 & 180.2 & 156.3 & 155.2 & 14,900 \\
\hline 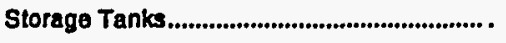 & 183.9 & 191.1 & 175.0 & 183.9 & 10,300 \\
\hline \multirow[t]{2}{*}{ 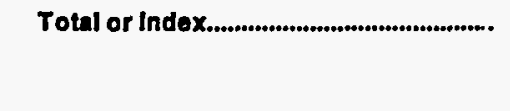 } & 192.4 & 196.4 & 172.7 & 178.3 & 54,200 \\
\hline & \multicolumn{5}{|c|}{5 Mlllion Cubic Feet Por Day } \\
\hline Flowlines and Connections............................ & 303.7 & 304.3 & 301.2 & 323.8 & 53,100 \\
\hline Production Package....................................... . & 146.4 & 150.0 & 129.1 & 123.6 & 13,600 \\
\hline 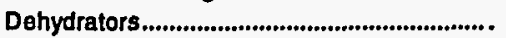 & 199.1 & 203.4 & 185.3 & 185.3 & 21,500 \\
\hline 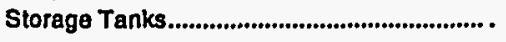 & 183.9 & 191.1 & 175.0 & 183.9 & 10,300 \\
\hline 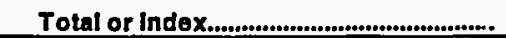 & 222.6 & 225.8 & 212.8 & 220.9 & 98,500 \\
\hline
\end{tabular}


Table J5. Lease Equipment Costs and Indices for Gas Production in South Louislana

(1 Well Producing from 16,000 Feet)

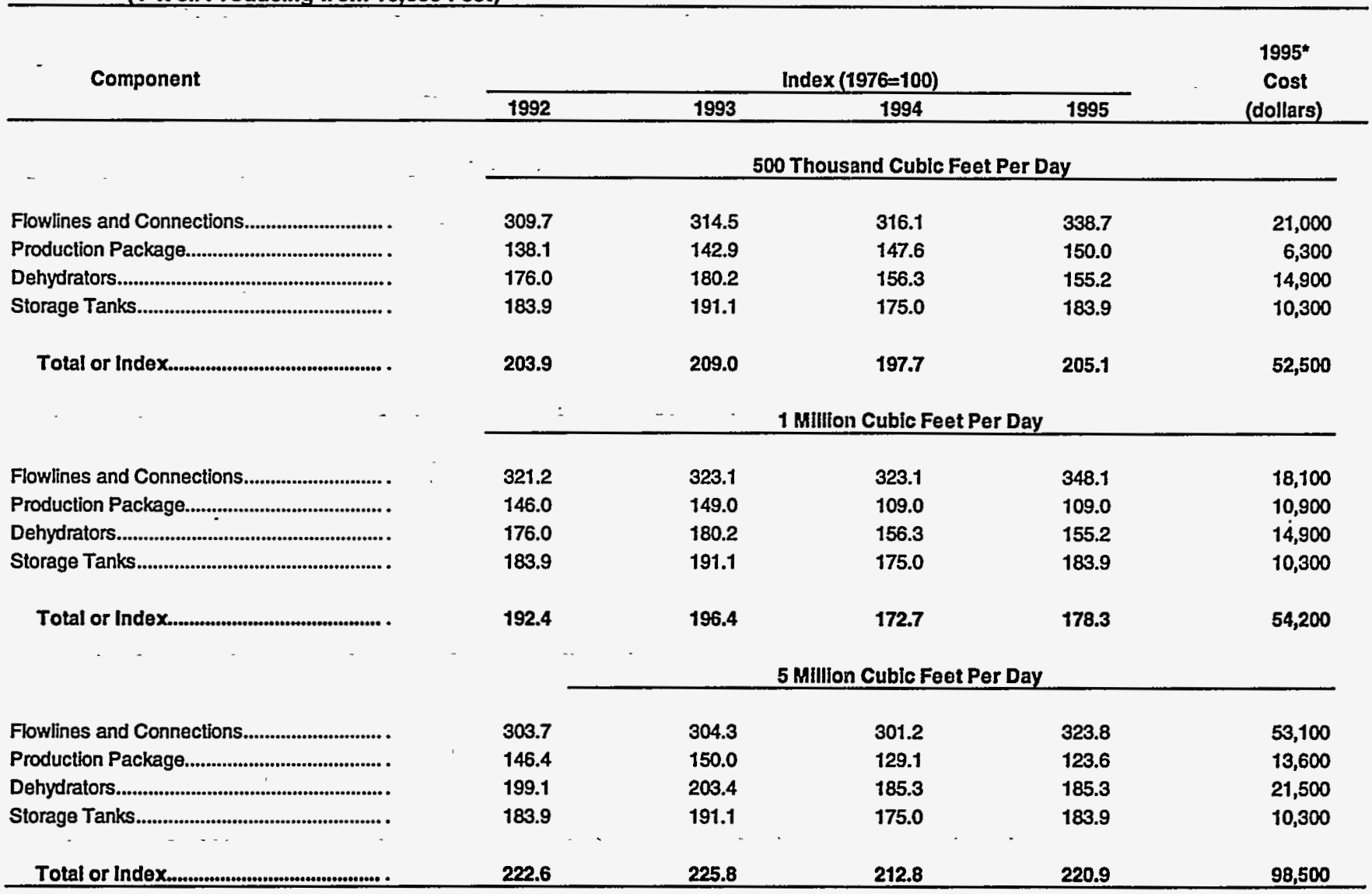

Table J6. Direct Annual Operating Costs and Indices for Gas Production in South Louisiana (1 Well Producing from 2,000 Feet)

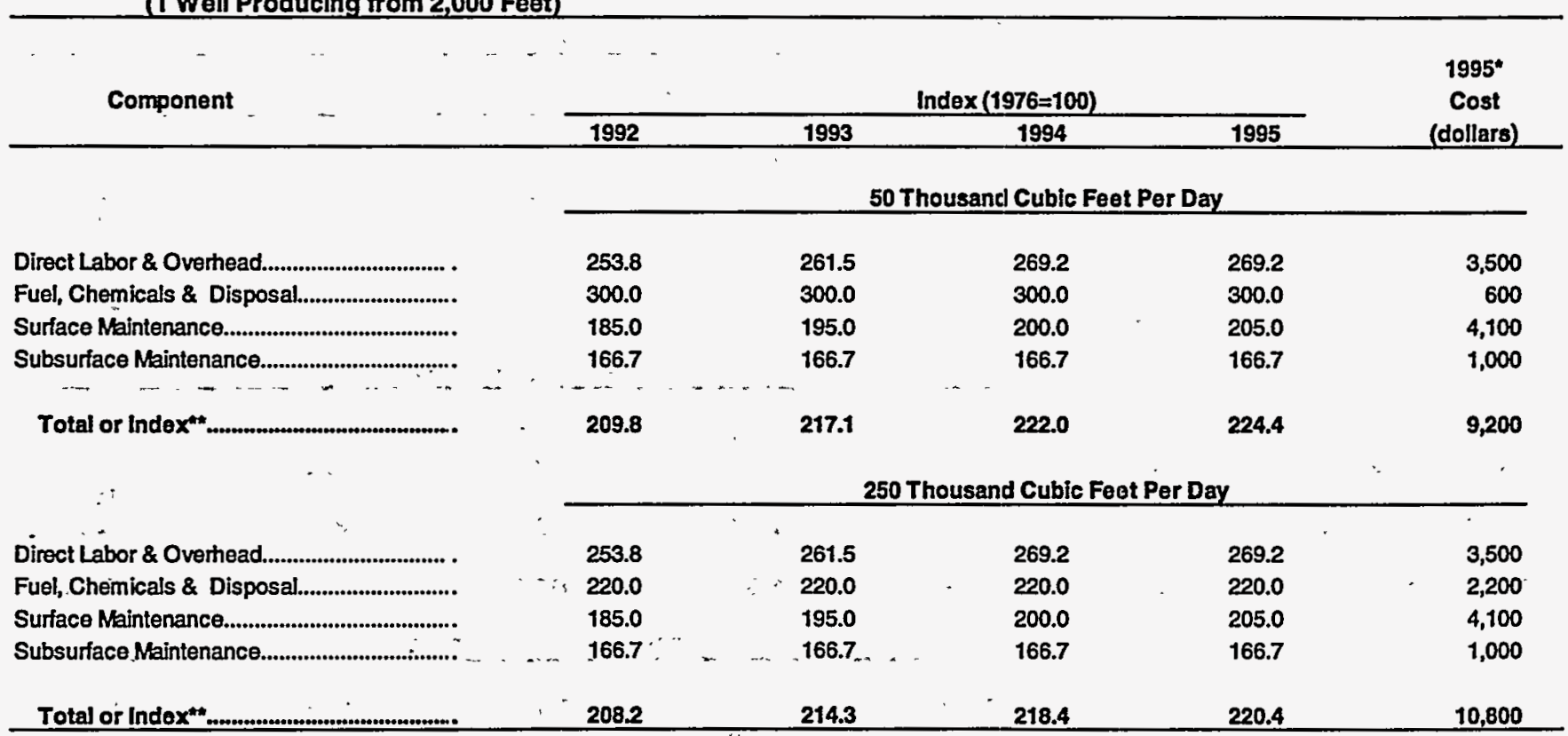


Table J7. Direct Annual Operating Costs and Indices for Gas Production in South Louisiana ( 1 Well Producing from 4,000 Feet)

\begin{tabular}{|c|c|c|c|c|c|}
\hline \multirow[t]{2}{*}{ Component } & \multicolumn{4}{|c|}{ Index $(1976=100)$} & \multirow{2}{*}{$\begin{array}{c}1995^{\star} \\
\text { Cost } \\
\text { (dollars) }\end{array}$} \\
\hline & 1992 & 1993 & 1994 & 1995 & \\
\hline & \multicolumn{5}{|c|}{50 Thousand Cubic Feet Per Day } \\
\hline Direct Labor \& Overhead................................. . & 264.3 & 271.4 & 278.6 & 285.7 & 4,000 \\
\hline Fuel, Chemicals \& Disposal........................... & 220.0 & 220.0 & 220.0 & 220.0 & 1,100 \\
\hline Surface Maintenance.................................... & 185.0 & 195.0 & 200.0 & 205.0 & 4,100 \\
\hline Subsurface Maintenance.................................. & 175.0 & 175.0 & 175.0 & 187.5 & 1,500 \\
\hline \multirow[t]{2}{*}{ Total or Index } & 210.6 & 217.0 & 221.3 & 227.7 & 10,700 \\
\hline & \multicolumn{5}{|c|}{250 Thousand Cubic Feet Per Day } \\
\hline Direct Labor \& Overhead................................. & 264.3 & 271.4 & 278.6 & 285.7 & 4,000 \\
\hline Fuel, Chemicals \& Disposal............................. & 220.0 & 220.0 & 220.0 & 220.0 & 4,400 \\
\hline Surface Maintenance...................................... & 192.0 & 200.0 & 200.0 & 204.0 & 5,100 \\
\hline Subsurface Maintenance.................................. & 175.0 & 175.0 & 175.0 & 187.5 & 1,500 \\
\hline Total or Index & 213.4 & 217.9 & 219.4 & 223.9 & 15,000 \\
\hline
\end{tabular}

Table J8. Direct Annual Operating Costs and Indices for Gas Production in South Loulsiana (1 Well Producing from 8,000 Feet)

\begin{tabular}{|c|c|c|c|c|c|}
\hline \multirow[t]{2}{*}{ Component } & \multicolumn{4}{|c|}{ Index $(1976=100)$} & \multirow{2}{*}{$\begin{array}{c}\text { 1995* } \\
\text { Cost } \\
\text { (dollars) }\end{array}$} \\
\hline & 1992 & 1993 & 1994 & 1995 & \\
\hline & \multicolumn{5}{|c|}{250 Thousand Cubic Feet Per Day } \\
\hline Direct Labor \& Ovehead................................. . & 252.9 & 258.8 & 264.7 & 270.6 & 4,600 \\
\hline Fuel, Chemicals \& Disposal.......................... & 225.6 & 225.6 & 225.6 & 225.6 & 8,800 \\
\hline Surface Malntenance...................................... & 184.0 & 192.0 & 194.0 & 196.0 & 9,800 \\
\hline Subsurface Maintenance.................................... & 191.7 & 191.7 & 191.7 & 200.0 & 2,400 \\
\hline \multirow[t]{2}{*}{ Total or Index" } & 208.5 & 212.7 & 214.4 & 216.9 & 25,600 \\
\hline & \multicolumn{5}{|c|}{500 Thousand Cubic Feet Per Day } \\
\hline Direct Labor \& Ovehead............................... . & 252.9 & 258.8 & 264.7 & 270.6 & 4,600 \\
\hline Fuel, Chemicals \& Disposal........................... & 200.0 & 207.7 & 211.5 & 215.4 & 5,600 \\
\hline Surface Maintenance & 187.8 & 195.9 & 198.0 & 200.0 & 9,800 \\
\hline Subsurface Maintenance.................................. & 191.7 & 191.7 & 191.7 & 200.0 & 2,400 \\
\hline \multirow[t]{2}{*}{ Total or Index } & 201.9 & 208.7 & 211.5 & 215.4 & 22,400 \\
\hline & \multicolumn{5}{|c|}{1 Million Cubic Feet Per Day } \\
\hline Direct Labor \& Overhead.................................. . & 252.9 & 258.8 & 264.7 & 270.6 & 4,600 \\
\hline 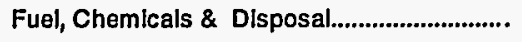 & 200.0 & 209.3 & 213.0 & 214.8 & 11,600 \\
\hline 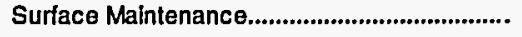 & 189.6 & 197.9 & 195.8 & 200.0 & 9,600 \\
\hline Subsurface Maintenance............................... & 191.7 & 191.7 & 191.7 & 200.0 & 2,400 \\
\hline Total or Index $x^{* *}$ & 202.3 & 209.9 & 211.5 & 215.3 & 28,200 \\
\hline
\end{tabular}


Table J9. Direct Annual Operating Costs and Indices for Gas Production in South Louislana (1 Well Producing from 12,000 Feet)

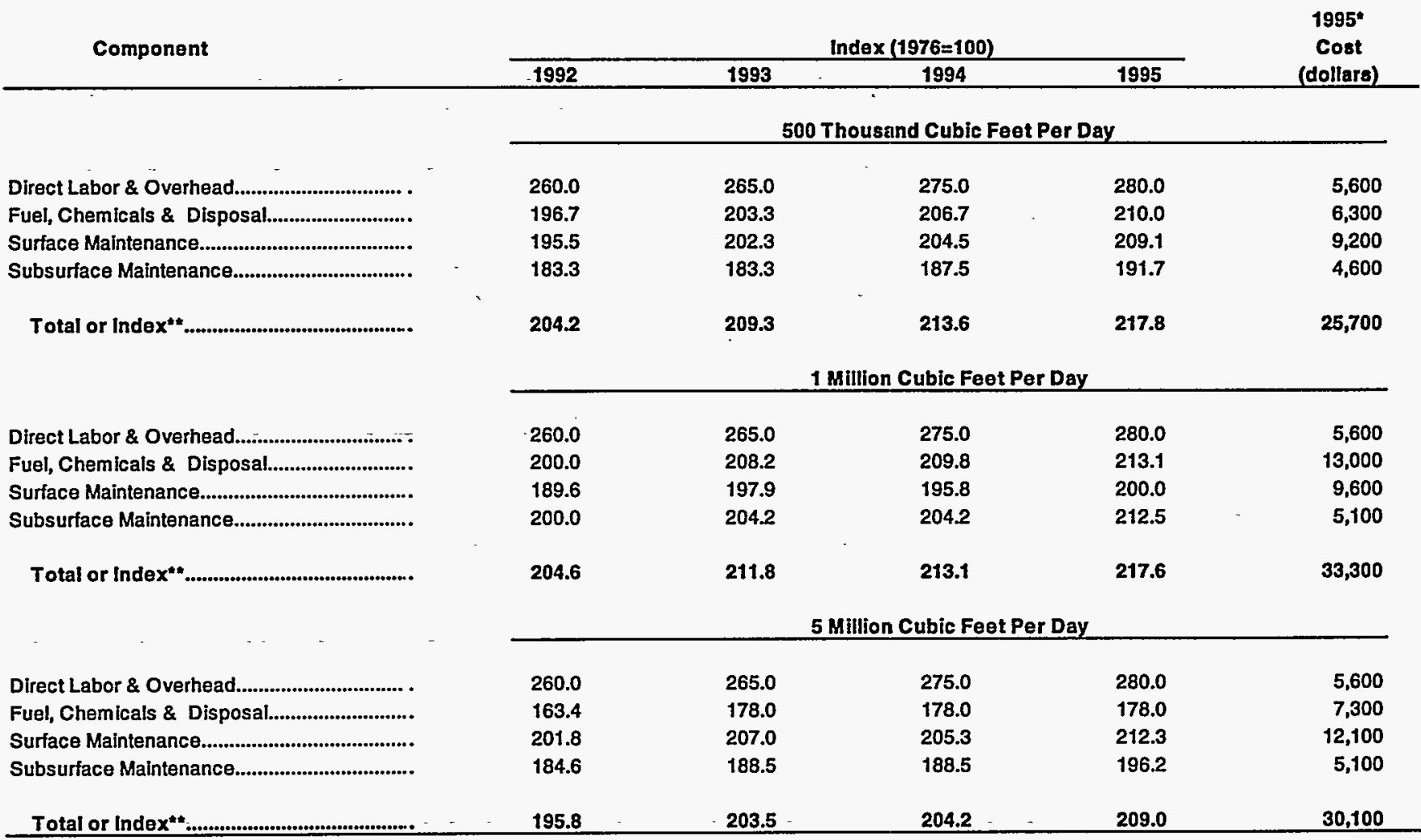

Table J10. Direct Annual Operating Costs and Indices for Gas Production in South Loulsiana (1 Well Producing from 16,000 Feet)

\begin{tabular}{|c|c|c|c|c|c|}
\hline \multirow[t]{2}{*}{ Component } & \multicolumn{4}{|c|}{ Index $(1976=100)$} & \multirow{2}{*}{$\begin{array}{c}\text { 1995* } \\
\text { Cost } \\
\text { (dollars) }\end{array}$} \\
\hline & 1992 & 1993 & 1994 & 1995 & \\
\hline & \multicolumn{5}{|c|}{500 Thousand Cubic Feet Per Day } \\
\hline Direct Labor \& Overhead.................................. . & 260.0 & 265.0 & 275.0 & 280.0 & 5,600 \\
\hline Fuel, Chemicals \& Disposal............................. & 191.4 & 197.1 & 200.0 & 202.9 & 7,100 \\
\hline Surface Maintenance......................................... & 195.5 & 202.3 & 204.5 & 209.1 & 9,200 \\
\hline Subsurface Maintenance................................. & 175.8 & 178.8 & 181.8 & 187.9 & 6,200 \\
\hline \multirow[t]{2}{*}{ 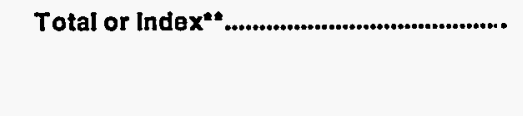 } & 199.2 & 204.5 & 208.3 & 212.9 & 28,100 \\
\hline & \multicolumn{5}{|c|}{1 Million Cubic Feet Per Day } \\
\hline Direct Labor \& Overhead.................................. . & 260.0 & 265.0 & 275.0 & 280.0 & 5,600 \\
\hline Fuel, Chemicals \& Disposal............................. & 197.2 & 202.8 & 205.6 & 208.5 & 14,800 \\
\hline Surface Maintenance........................................ & 189.6 & 197.9 & 195.8 & 200.0 & 9,600 \\
\hline Subsurface Maintenance................................. & 175.8 & 178.8 & 181.8 & 187.9 & 6,200 \\
\hline \multirow[t]{2}{*}{ Total or Index } & 198.3 & 204.1 & 206.4 & 210.5 & 36,200 \\
\hline & \multicolumn{4}{|c|}{5 Million Cubic Feet Per Day } & \\
\hline Direct Labor \& Overhead................................... . & $-\quad 260.0$ & 265.0 & 275.0 & 280.0 & 5,600 \\
\hline Fuel, Chemicals \& Disposal.............................. & 149.5 & 156.8 & 158.9 & 161.1 & 15,300 \\
\hline Surface Maintenance.......................................... & 194.9 & 200.0 & 198.3 & 205.1 & 12,100 \\
\hline Subsurface Maintenance.................................... & 177.1 & 182.9 & 182.9 & 188.6 & 6,600 \\
\hline 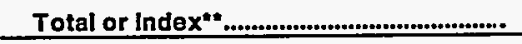 & 177.5 & 183.7 & 185.2 & 189.5 & 39,600 \\
\hline
\end{tabular}


Table K1. Lease Equipment Costs and Indices for Gas Production in North Louisiana (1 Well Producing from 2,000 Feet)

\begin{tabular}{|c|c|c|c|c|c|}
\hline \multirow[t]{2}{*}{ Component } & \multicolumn{4}{|c|}{ Index $(1976=100)$} & \multirow{2}{*}{$\begin{array}{c}\text { 1995" } \\
\text { Cost } \\
\text { (dollars) }\end{array}$} \\
\hline & 1992 & 1993 & 1994 & 1995 & \\
\hline & \multicolumn{5}{|c|}{ 50 Thousand Cublc Feet Per Day } \\
\hline Flowlines and Connections............................. . & 150.0 & 164.3 & 164.3 & 178.6 & 2,500 \\
\hline Production Package....................................... & 148.6 & 151.4 & 160.0 & 162.9 & 5,700 \\
\hline 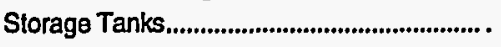 & 183.9 & 191.1 & 175.0 & 183.9 & 10,300 \\
\hline \multirow[t]{2}{*}{ Total or Index........................................ } & 167.6 & 174.3 & 168.6 & 176.2 & 18,500 \\
\hline & \multicolumn{5}{|c|}{ 250 Thousand Cubic Feet Per Day } \\
\hline Flowlines and Connections............................. & 150.0 & 164.3 & 164.3 & 178.6 & 2,500 \\
\hline Production Package....................................... & 148.6 & 151.4 & 160.0 & 162.9 & 5,700 \\
\hline Storage Tanks.................................................... & 183.9 & 191.1 & 175.0 & 183.9 & 10,300 \\
\hline Total or Index............................................. . & 167.6 & 174.3 & 168.6 & 176.2 & 18,500 \\
\hline
\end{tabular}

Table K2. Lease Equipment Costs and Indices for Gas Production in North Louisiana (1 Well Producing from 4,000 Feet)

\begin{tabular}{|c|c|c|c|c|c|}
\hline \multirow[t]{2}{*}{ Component } & \multicolumn{4}{|c|}{ Index $(1976=100)$} & \multirow{2}{*}{$\begin{array}{c}\text { 1995" } \\
\text { Cost } \\
\text { (dollars) }\end{array}$} \\
\hline & 1992 & 1993 & 1994 & 1995 & \\
\hline & \multicolumn{5}{|c|}{250 Thousand Cubic Feet Per Day } \\
\hline Flowlines and Connections........................... & 208.7 & 213.0 & 184.8 & 195.7 & 9,000 \\
\hline 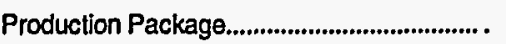 & 167.6 & 175.7 & 178.4 & 186.5 & 6,900 \\
\hline Storage Tanks................................................ & 183.9 & 191.1 & 175.0 & 183.9 & 10,300 \\
\hline \multirow[t]{2}{*}{ Total or Index......................................... } & 187.8 & 194.2 & 179.1 & 188.5 & 26,200 \\
\hline & \multicolumn{5}{|c|}{500 Thousand Cubic Feet Per Day } \\
\hline Flowlines and Connections............................ & 250.0 & 283.3 & 283.3 & 300.0 & 1,800 \\
\hline Production Package & 138.1 & 142.9 & 147.6 & 150.0 & 6,300 \\
\hline 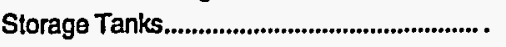 & 183.9 & 191.1 & 175.0 & 183.9 & 10,300 \\
\hline 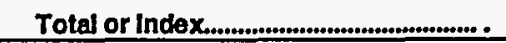 & 169.2 & 176.9 & 170.2 & 176.9 & 18,400 \\
\hline
\end{tabular}


Table K3. Lease Equipment Costs and Indices for Gas Production in North Louisiana (1 Well Producing from 8,000 Feet)

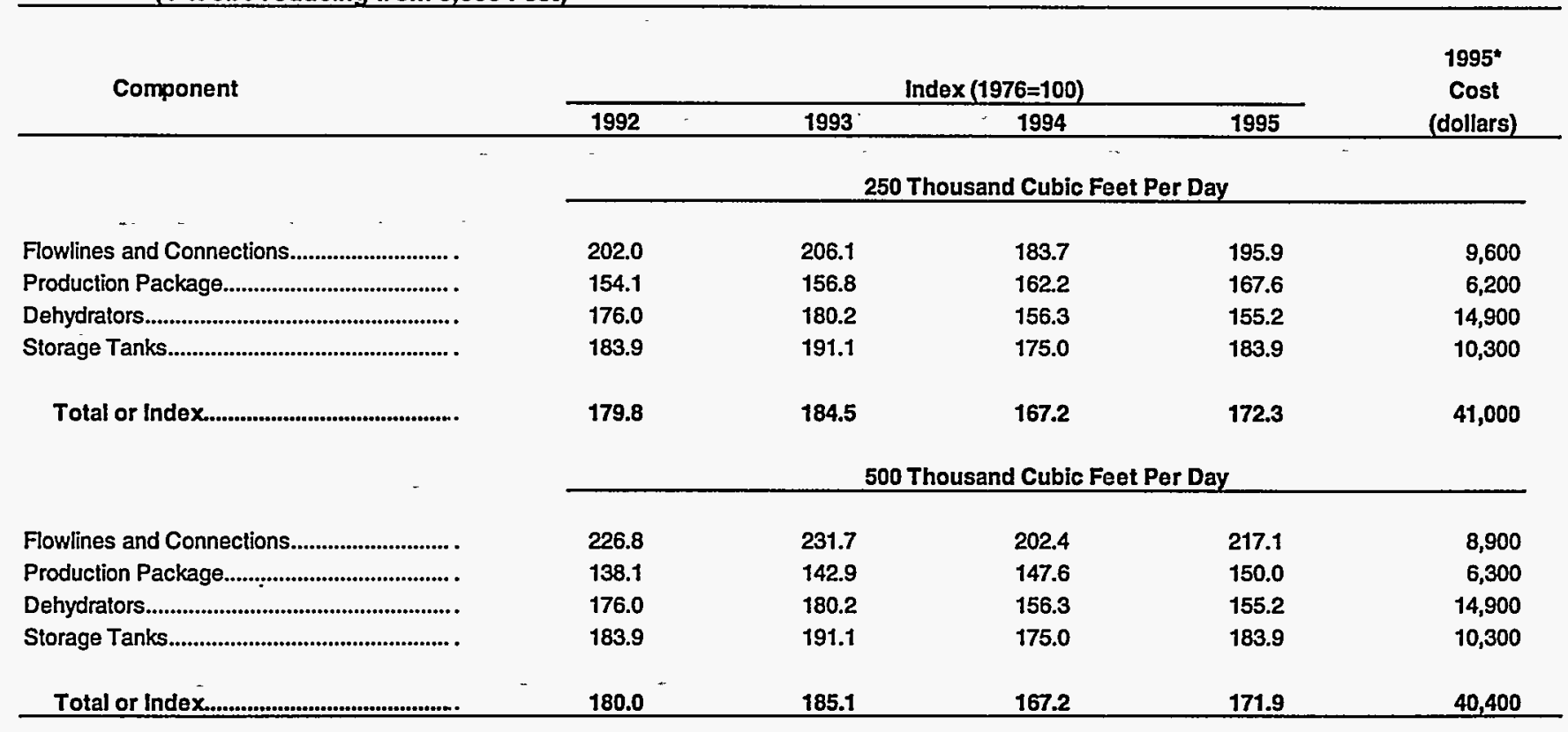

Table K4. Lease Equipment Costs and Indices for Gas Production in North Louisiana (1 Well Producing from 12,000 Feet)

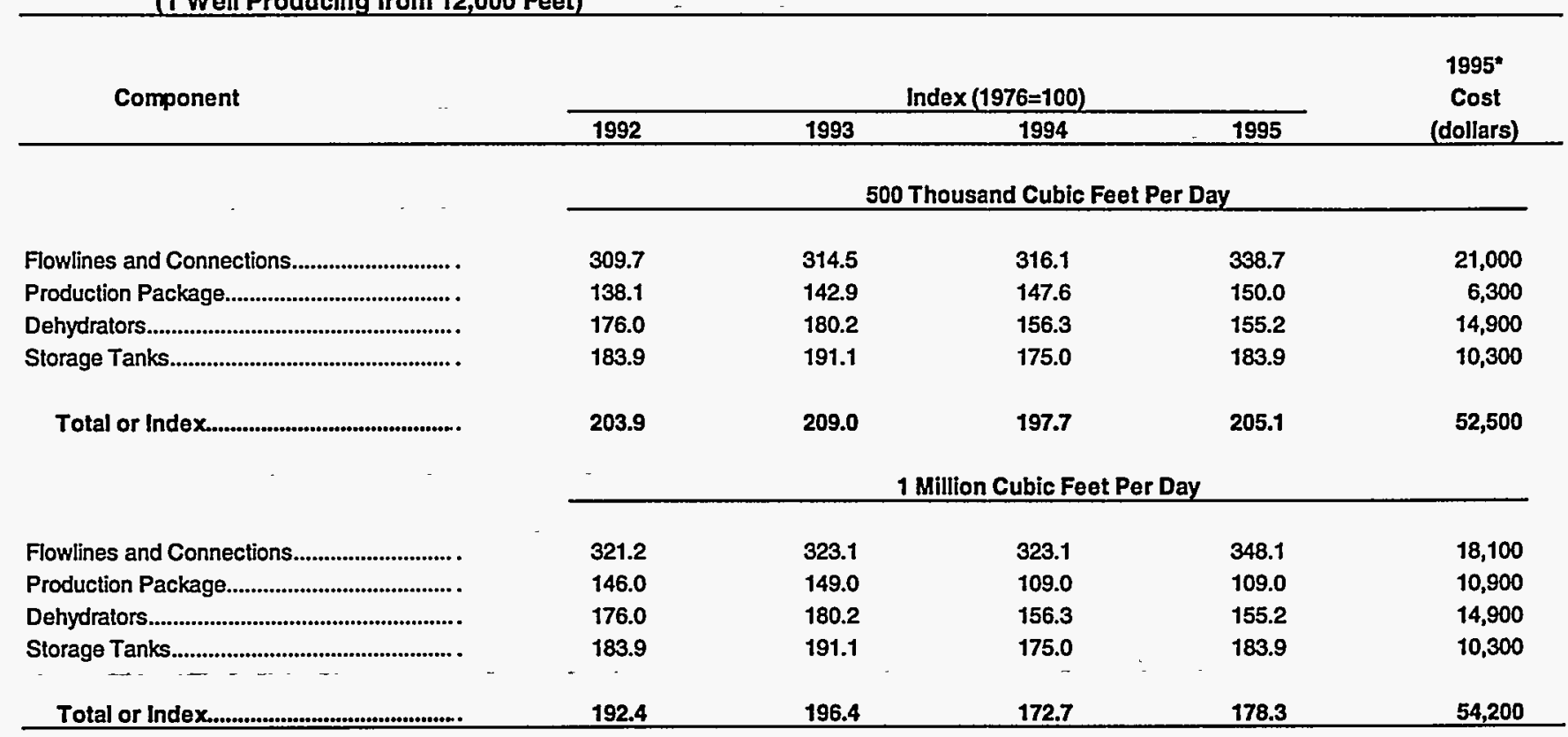


Table K5. Lease Equipment Costs and Indices for Gas Production in North Louisiana (1 Well Producing from 16,000 Feet)

\begin{tabular}{|c|c|c|c|c|c|}
\hline \multirow[t]{2}{*}{ Component } & \multicolumn{4}{|c|}{ Index $(1976=100)$} & \multirow{2}{*}{$\begin{array}{c}\text { 1995* } \\
\text { Cost } \\
\text { (dollars) }\end{array}$} \\
\hline & 1992 & 1993 & 1994 & 1995 & \\
\hline & \multicolumn{5}{|c|}{1 Million Cuble Feet Per Day } \\
\hline Flowlines and Connections............................. & 321.2 & 323.1 & 323.1 & 348.1 & 18,100 \\
\hline Production Package & 146.0 & 149.0 & 109.0 & 109.0 & 10,900 \\
\hline 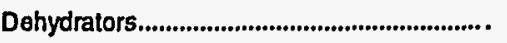 & 176.0 & 180.2 & 156.3 & 155.2 & 14,900 \\
\hline 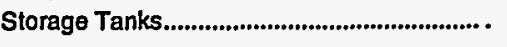 & 183.9 & 191.1 & 175.0 & 183.9 & 10,300 \\
\hline \multirow[t]{2}{*}{ Total or Index.......................................... . } & 192.4 & 196.4 & 172.7 & 178.3 & 54,200 \\
\hline & \multicolumn{5}{|c|}{5 Million Cubic Feet Per Day } \\
\hline Flowlines and Connections............................. & 303.7 & 304.3 & 301.2 & 323.8 & 53,100 \\
\hline Production Package & 146.4 & 150.0 & 129.1 & 123.6 & 13,600 \\
\hline 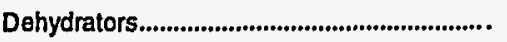 & 199.1 & 203.4 & 185.3 & 185.3 & 21,500 \\
\hline 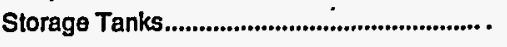 & 183.9 & 191.1 & 175.0 & 183.9 & 10,300 \\
\hline \multirow[t]{2}{*}{ Total or Index........................................ . } & 222.6 & 225.8 & 212.8 & 220.9 & 98,500 \\
\hline & \multicolumn{5}{|c|}{10 Million Cuble Feet Per Day } \\
\hline Flowlines and Connections............................ & 303.7 & 304.3 & 301.2 & 323.8 & 53,100 \\
\hline Production Package............................................ . & 146.4 & 150.0 & 129.1 & 123.6 & 13,600 \\
\hline 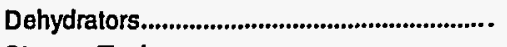 & 190.1 & 194.3 & 208.4 & 208.7 & 54,900 \\
\hline 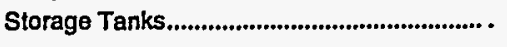 & 183.9 & 191.1 & 175.0 & 183.9 & 10,300 \\
\hline 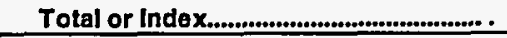 & 212.8 & 216.2 & 216.2 & 222.4 & 131,900 \\
\hline
\end{tabular}

Table K6. Direct Annual Operating Costs and Indices for Gas Production in North Louisiana (1 Well Producing from 2,000 Feet)

\begin{tabular}{|c|c|c|c|c|c|}
\hline \multirow[t]{2}{*}{ Component } & \multicolumn{4}{|c|}{ Index $(1976=100)$} & \multirow{2}{*}{$\begin{array}{c}\text { 1995* } \\
\text { Cost } \\
\text { (dollars) }\end{array}$} \\
\hline & 1992 & 1993 & 1994 & 1995 & \\
\hline & \multicolumn{5}{|c|}{50 Thousand Cubic Feet Per Day } \\
\hline Direct Labor \& Overhead.................................. . & 253.8 & 261.5 & 269.2 & 269.2 & 3,500 \\
\hline Fuel, Chemicals \& Disposal............................ & 250.0 & 250.0 & 250.0 & 250.0 & 500 \\
\hline Surlace Maintenance....................................... & 185.0 & 195.0 & 200.0 & 205.0 & 4,100 \\
\hline Subsurface Maintenance............................... & 166.7 & 166.7 & 166.7 & 166.7 & 1,000 \\
\hline \multirow[t]{2}{*}{ Total or Index } & 207.3 & 214.6 & 219.5 & 222.0 & 9,100 \\
\hline & \multicolumn{5}{|c|}{250 Thousand Cubic Feet Per Day } \\
\hline Direct Labor \& Overhead.................................. . & 253.8 & 261.5 & 269.2 & 269.2 & 3,500 \\
\hline Fuel, Chemicals \& Disposal............................. & 210.0 & 210.0 & 210.0 & 210.0 & 2,100 \\
\hline 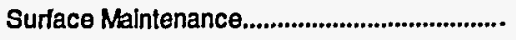 & 185.0 & 195.0 & 200.0 & 205.0 & 4,100 \\
\hline Subsurface Maintenance................................... & 166.7 & 166.7 & 166.7 & 166.7 & 1,000 \\
\hline Total or Index ${ }^{* \star} \ldots \ldots \ldots \ldots$ & 206.1 & 212.2 & 216.3 & 218.4 & 10,700 \\
\hline
\end{tabular}


Table K7. Direct Annual Operating Costs and Indices for Gas Production in North Louislana (1 Well Producing from 4,000 Feet)

\begin{tabular}{|c|c|c|c|c|c|}
\hline \multirow[t]{2}{*}{ Component } & \multicolumn{4}{|c|}{ Index $(1976=100)$} & \multirow{2}{*}{$\begin{array}{c}1995^{\star} \\
\text { Cost } \\
\text { (dollars) }\end{array}$} \\
\hline & 1992 & 1993 & 1994 & 1995 & \\
\hline-- & \multicolumn{5}{|c|}{250 Thousand Cubic Feet Per Day } \\
\hline Direct Labor \& Overhead.................................... . & 264.3 & 271.4 & 278.6 & 285.7 & 4,000 \\
\hline 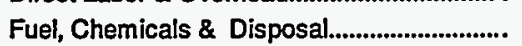 & 205.0 & 205.0 & 205.0 & 205.0 & 4,100 \\
\hline 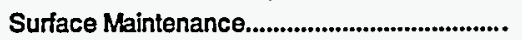 & 192.0 & 200.0 & 200.0 & 204.0 & 5,100 \\
\hline 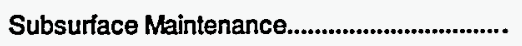 & 175.0 & 175.0 & 175.0 & 175.0 & 1,400 \\
\hline \multirow[t]{2}{*}{ 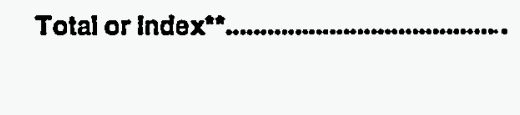 } & 209.0 & 213.4 & 214.9 & 217.9 & 14,600 \\
\hline & \multicolumn{5}{|c|}{500 Thousand Cuble Foet Per Day } \\
\hline Direct Labor \& Overhead................................... . & 264.3 & 271.4 & 278.6 & 285.7 & 4,000 \\
\hline 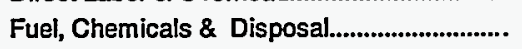 & 187.5 & 195.8 & 200.0 & 200.0 & 4,800 \\
\hline 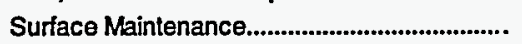 & 192.0 & 200.0 & 200.0 & 204.0 & 5,100 \\
\hline 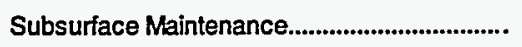 & 175.0 & 175.0 & 175.0 & 175.0 & 1,400 \\
\hline Total or Index & 202.8 & 209.9 & 212.7 & 215.5 & 15,300 \\
\hline
\end{tabular}

Table K8. Direct Annual Operating Costs and Indices for Gas Production in North Louisiana (1 Well Producing from 8,000 Feet)

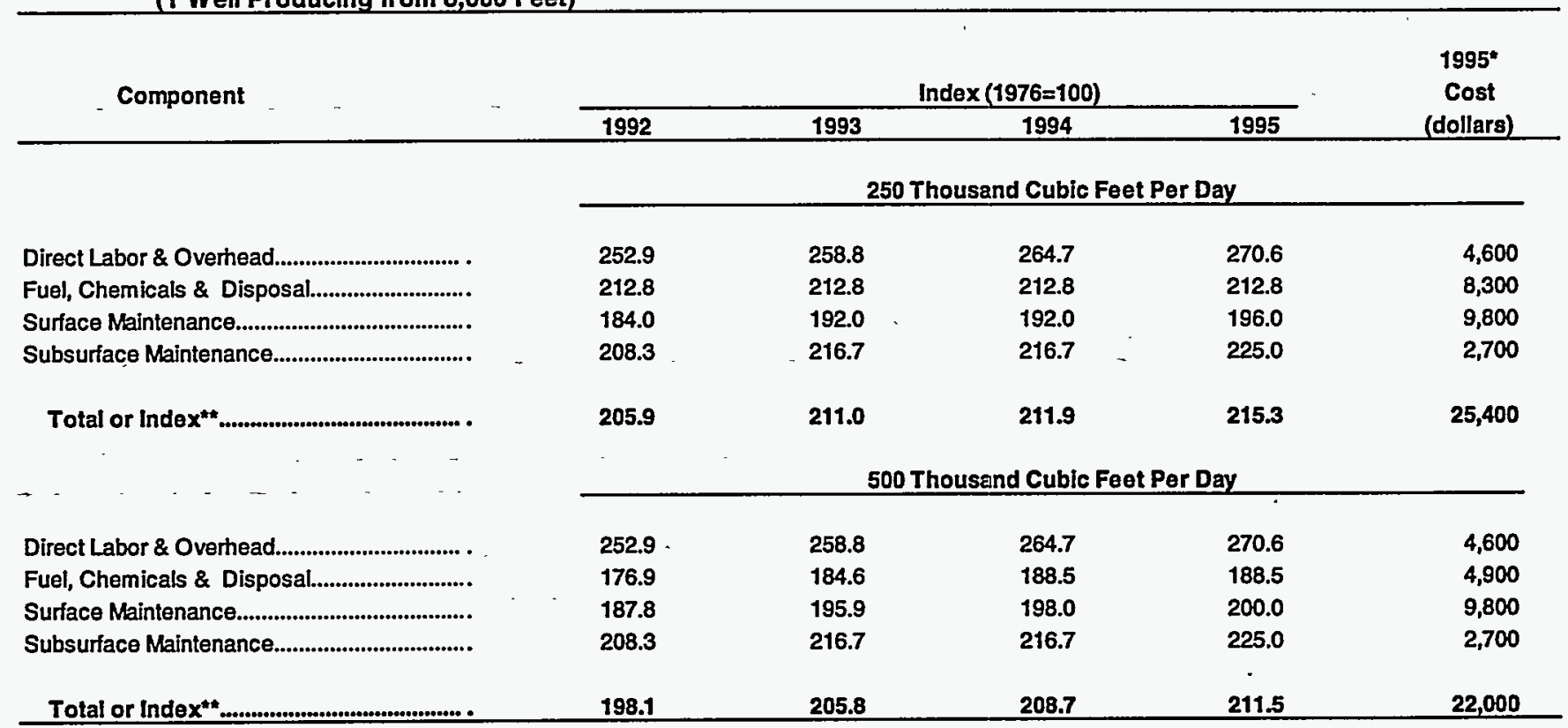


Table K9. Direct Annual Operating Costs and Indices for Gas Production in North Louisiana (1 Well Producing from 12,000 Feet)

\begin{tabular}{|c|c|c|c|c|c|}
\hline \multirow[t]{2}{*}{ Component } & \multicolumn{4}{|c|}{ Index $(1976=100)$} & \multirow{2}{*}{$\begin{array}{c}1995^{\circ} \\
\text { Cost } \\
\text { (dollars) }\end{array}$} \\
\hline & 1992 & 1993 & 1994 & 1995 & \\
\hline & \multicolumn{5}{|c|}{500 Thousand Cubic Feet Per Day } \\
\hline Direct Labor \& Overhead............................... . & 260.0 & 265.0 & 275.0 & 280.0 & 5,600 \\
\hline Fuel, Chemicals \& Disposal........................... & 190.0 & 196.7 & 200.0 & 203.3 & 6,100 \\
\hline Surface Maintenance..................................... & 195.5 & 202.3 & 204.5 & 209.1 & 9,200 \\
\hline Subsurface Maintenance.................................. & 141.7 & 145.8 & 145.8 & 154.2 & 3,700 \\
\hline \multirow[t]{2}{*}{ 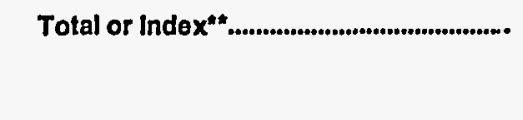 } & 194.1 & 200.0 & 203.4 & 208.5 & 24,600 \\
\hline & \multicolumn{5}{|c|}{1 Million Cubic Feet Per Day } \\
\hline Direct Labor \& Overhead................................. . & 260.0 & 265.0 & 275.0 & 280.0 & 5,600 \\
\hline Fuel, Chemicals \& Disposal............................ & 196.7 & 204.9 & 206.6 & 209.8 & 12,800 \\
\hline 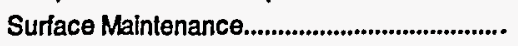 & 189.6 & 197.9 & 195.8 & 200.0 & 9,600 \\
\hline Subsurface Maintenance.................................. & 141.7 & 145.8 & 145.8 & 154.2 & 3,700 \\
\hline Total or Index & 194.1 & 201.3 & 202.6 & 207.2 & 31,700 \\
\hline
\end{tabular}

Table K10. Direct Annual Operating Costs and Indices for Gas Production in North Louisiana

(1 Well Producing from 16,000 Feet)

\begin{tabular}{|c|c|c|c|c|c|}
\hline \multirow[t]{2}{*}{ Component } & \multicolumn{4}{|c|}{ Index $(1976=100)$} & \multirow{2}{*}{$\begin{array}{c}1995^{\circ} \\
\text { Cost } \\
\text { (dollars) }\end{array}$} \\
\hline & 1992 & 1993 & 1994 & 1995 & \\
\hline & & - & & & \\
\hline & \multicolumn{5}{|c|}{1 Million Cuble Feet Per Day } \\
\hline Direct Labor \& Overhead................................ . & 260.0 & 265.0 & 275.0 & 280.0 & 5,600 \\
\hline Fuel, Chemicals \& Disposal............................ & 193.0 & 200.0 & 202.8 & 204.2 & 14,500 \\
\hline Surface Malntenance........................................ & 189.6 & 195.8 & 193.8 & 197.9 & 9,500 \\
\hline Subsurface Maintenance................................ & 175.8 & 178.8 & 181.8 & 187.9 & 6,200 \\
\hline \multirow[t]{2}{*}{ Total or Index } & 196.5 & 202.3 & 204.7 & 208.1 & 35,800 \\
\hline & \multicolumn{5}{|c|}{5 Million Cubic Feet Per Day } \\
\hline Direct Labor \& Overhead................................. . & 260.0 & 265.0 & 275.0 & 280.0 & 5,600 \\
\hline Fuel, Chemicals \& Disposal............................ & 150.0 & 158.3 & 160.4 & 162.5 & 15,600 \\
\hline Surface Maintenance........................................ & 194.9 & 200.0 & 196.6 & 203.4 & 12,000 \\
\hline Subsurface Maintenance.................................. & 177.1 & 182.9 & 182.9 & 188.6 & 6,600 \\
\hline \multirow[t]{2}{*}{ Total or Index**....................................... } & 177.6 & 184.3 & 185.2 & 189.5 & 39,800 \\
\hline & \multicolumn{5}{|c|}{10 Million Cubic Feet Per Day } \\
\hline Direct Labor \& Overhead................................. . & 260.0 & 265.0 & 275.0 & 280.0 & 5,600 \\
\hline Fuel, Chemicals \& Disposal........................... & 146.0 & 153.4 & 155.8 & 157.1 & 25,600 \\
\hline Surface Maintenance.......................................... & 198.6 & 202.9 & 207.2 & 213.0 & 14,700 \\
\hline Subsurface Maintenance................................... & 177.1 & 182.9 & 182.9 & 188.6 & 6,600 \\
\hline Total or Index & 170.4 & 176.7 & 179.8 & 182.9 & 52,500 \\
\hline
\end{tabular}


Table L1. Lease Equipment Costs and Indices for Gas Production in the Mid-Continent (1 Well Producing from 2,000 Feet)

\begin{tabular}{|c|c|c|c|c|c|}
\hline \multirow{2}{*}{ Component } & \multicolumn{2}{|r|}{$\sim$} & \multicolumn{2}{|c|}{ Index $(1976=100)$} & \multirow{2}{*}{$\begin{array}{c}1995^{\star} \\
\text { Cost } \\
\text { (dollars) }\end{array}$} \\
\hline & 1992 & -1993 & 1994 & 1995 & \\
\hline & \multicolumn{4}{|c|}{50 Thousand Cubic Feat Per Day } & \\
\hline Flowlines and Connections............................. & 169.2 & 184.6 & 184.6 & 192.3 & 2,500 \\
\hline Production Package........................................ . & 152.9 & 158.8 & 164.7 & 164.7 & 5,600 \\
\hline 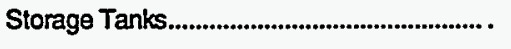 & 192.6 & 200.0 & 183.3 & 192.6 & 10,400 \\
\hline \multirow[t]{2}{*}{ Total or Index } & 176.2 & 184.2 & 177.2 & 183.2 & 18,500 \\
\hline & \multicolumn{5}{|c|}{250 Thousand Cubic Feet Per Day } \\
\hline Flowlines and Connections............................. & 169.2 & 184.6 & 184.6 & 192.3 & 2,500 \\
\hline 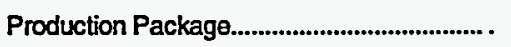 & 147.5 & 152.5 & 157.5 & 157.5 & 6,300 \\
\hline 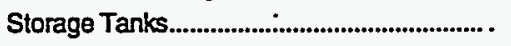 & 192.6 & 200.0 & 183.3 & 192.6 & 10,400 \\
\hline Total or Index & 172.9 & 180.4 & 173.8 & 179.4 & 19,200 \\
\hline
\end{tabular}

Table L2. Lease Equipment Costs and Indices for Gas Production in the Mid-Continent (1 Well Producing from 4,000 Feet)

\begin{tabular}{|c|c|c|c|c|c|}
\hline \multirow[t]{2}{*}{ Component } & \multicolumn{4}{|c|}{ Index $(1976=100)$} & \multirow{2}{*}{$\begin{array}{c}\text { 1995* } \\
\text { Cost } \\
\text { (dollars) }\end{array}$} \\
\hline & 1992 & 1993 & 1994 & 1995 & \\
\hline & \multicolumn{5}{|c|}{ 50 Thousand Cubic Feet Per Day } \\
\hline Flowlines and Connections............................ & 169.2 & 184.6 & 184.6 & 192.3 & 2,500 \\
\hline Production Package........................................ . & 152.9 & 158.8 & 164.7 & 164.7 & 5,600 \\
\hline Storage Tanks................................................ & 192.6 & 200.0 & 183.3 & 192.6 & 10,400 \\
\hline \multirow[t]{2}{*}{ Total or Index......................................... } & 176.2 & 184.2 & 177.2 & 183.2 & 18,500 \\
\hline & \multicolumn{4}{|c|}{ 250 Thousand Cubic Feet Per Day } & \\
\hline Flowlines and Connections............................ . & 213.3 & 220.0 & 188.9 & 202.2 & 9,100 \\
\hline Production Package.......................................... & 165.7 & 171.4 & 174.3 & 177.1 & 6,200 \\
\hline Storage Tanks................................................. & 192.6 & 200.0 & 183.3 & 192.6 & 10,400 \\
\hline \multirow[t]{2}{*}{ 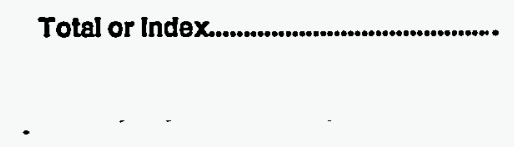 } & 192.5 & 199.3 & 182.8 & 191.8 & 25,700 \\
\hline & \multicolumn{5}{|c|}{500 Thousand Cubic Feet Per Day } \\
\hline Fowlines and Connections............................ & 236.8 & 242.1 & 205.3 & 221.1 & 8,400 \\
\hline Production Package....................................... . & 165.7 & 171.4 & 174.3 & 177.1 & 6,200 \\
\hline Storage Tanks................................................... . & 192.6 & 200.0 & 183.3 & 192.6 & 10,400 \\
\hline Total or Index.................................................. & 198.4 & 204.7 & 187.4 & 196.9 & 25,000 \\
\hline
\end{tabular}


Table L3. Lease Equlpment Costs and Indices for Gas Production In the Mid-Continent (1 Well Producing from 8,000 Feet)

\begin{tabular}{|c|c|c|c|c|c|}
\hline \multirow[t]{2}{*}{ Component } & \multicolumn{4}{|c|}{ Index $(1976=100)$} & \multirow{2}{*}{$\begin{array}{c}1995^{*} \\
\text { Cost } \\
\text { (dollars) }\end{array}$} \\
\hline & 1992 & 1993 & 1994 & 1995 & \\
\hline & \multicolumn{5}{|c|}{250 Thousand Cubic Feet Per Day } \\
\hline Flowlines and Connections.............................. . & 206.3 & 212.5 & 187.5 & 200.0 & 9,600 \\
\hline Production Package............................................. . & 165.7 & 171.4 & 174.3 & 177.1 & 6,200 \\
\hline 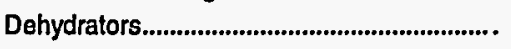 & 184.9 & 189.2 & 163.4 & 161.3 & 15,000 \\
\hline 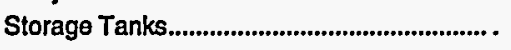 & 192.6 & 200.0 & 183.3 & 192.6 & 10,400 \\
\hline \multirow[t]{2}{*}{ 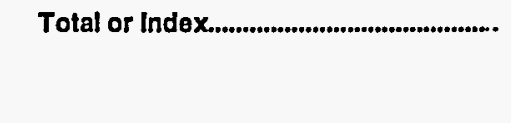 } & 188.3 & 193.9 & 174.8 & 179.1 & 41,200 \\
\hline & \multicolumn{5}{|c|}{500 Thousand Cubic Feet Per Day } \\
\hline Flowlines and Connections................................ . & 226.8 & 231.7 & 202.4 & 217.1 & 8,900 \\
\hline Production Package............................................... & 175.0 & 177.5 & 185.0 & 185.0 & 7,400 \\
\hline 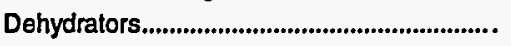 & 184.9 & 189.2 & 163.4 & 161.3 & 15,000 \\
\hline 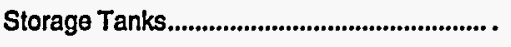 & 192.6 & 200.0 & 183.3 & 192.6 & 10,400 \\
\hline Total or Index & 192.5 & 197.4 & 178.9 & 182.9 & 41,700 \\
\hline
\end{tabular}

Table L4. Lease Equipment Costs and Indices for Gas Production In the Mid-Continent (1 Well Producing from 12,000 Feet)

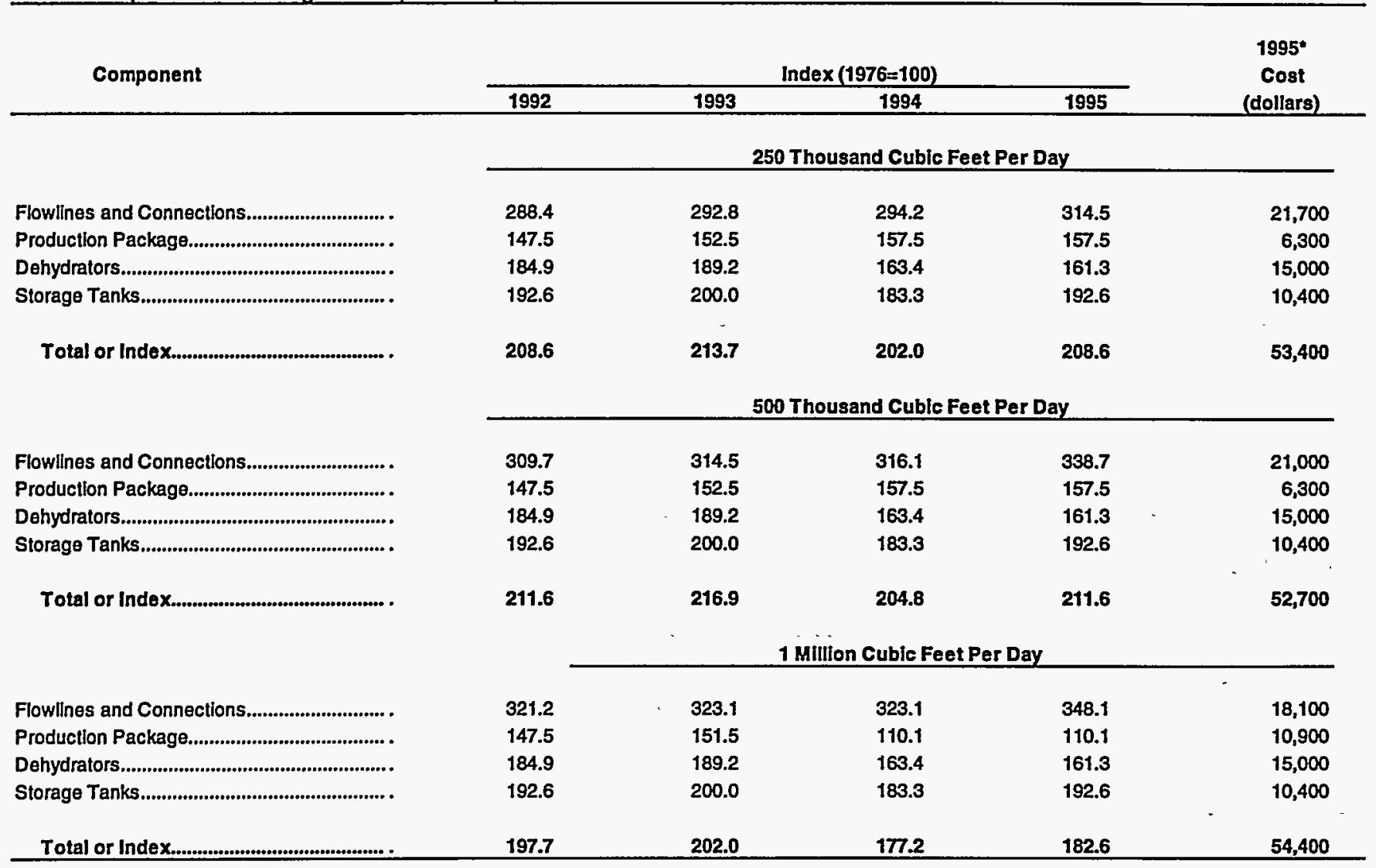


Table L5. Lease Equipment Costs and Indices for Gas Production in the Mid-Continent (1 Well Producing from 16,000 Feet)

\begin{tabular}{|c|c|c|c|c|c|}
\hline \multirow{2}{*}{ Component } & \multicolumn{4}{|c|}{ Index $(1976=100)$} & \multirow{2}{*}{$\begin{array}{c}\text { 1995* } \\
\text { Cost } \\
\text { (dollars) }\end{array}$} \\
\hline & 1992 & 1993 & 1994 & 1995 & \\
\hline & \multicolumn{5}{|c|}{500 Thousand Cubic Feet Per Day } \\
\hline Flowlines and Connections............................. & 321.2 & 323.1 & 323.1 & 348.1 & 18,100 \\
\hline 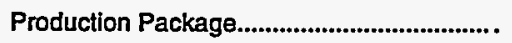 & 147.5 & 151.5 & 110.1 & 110.1 & 10,900 \\
\hline 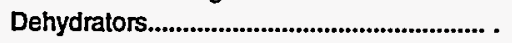 & 184.9 & 189.2 & 163.4 & 161.3 & 15,000 \\
\hline 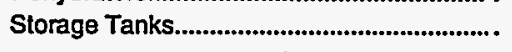 & 192.6 & 200.0 & 183.3 & 192.6 & 10,400 \\
\hline \multirow{2}{*}{ Total or Index................................................... } & 197.7 & 202.0 & 177.2 & 182.6 & 54,400 \\
\hline & \multicolumn{5}{|c|}{1 Million Cubic Feet Per Day } \\
\hline Flowlines and Connections............................ & 321.2 & 323.1 & 323.1 & 348.1 & 18,100 \\
\hline 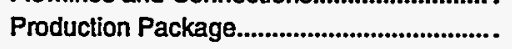 & 147.5 & 151.5 & 110.1 & 110.1 & 10,900 \\
\hline 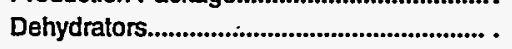 & 184.9 & 189.2 & 163.4 & 161.3 & 15,000 \\
\hline 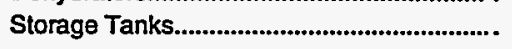 & 192.6 & 200.0 & 183.3 & 192.6 & 10,400 \\
\hline 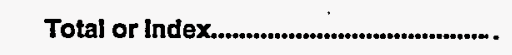 & 197.7 & 202.0 & 177.2 & 182.6 & 54,400 \\
\hline - & \multicolumn{5}{|c|}{5 Million Cubic Feet Per Day } \\
\hline Flowlines and Connections........................... . & 303.7 & 304.3 & 301.2 & 323.8 & 53,100 \\
\hline 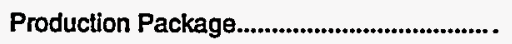 & 149.1 & 152.8 & 131.5 & 125.0 & 13,500 \\
\hline 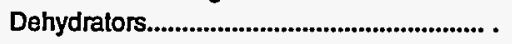 & 206.2 & 210.6 & 192.0 & 192.0 & 21,700 \\
\hline 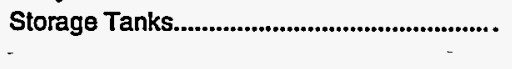 & 192.6 & 200.0 & 183.3 & 192.6 & 10,400 \\
\hline Total or Index & 226.9 & 230.1 & 216.9 & 224.8 & 98,700 \\
\hline
\end{tabular}

Table L6. Direct Annual Operating Costs and Indices for Gas Production in the Mid-Continent (1 Well Producing from 2,000 Feet)

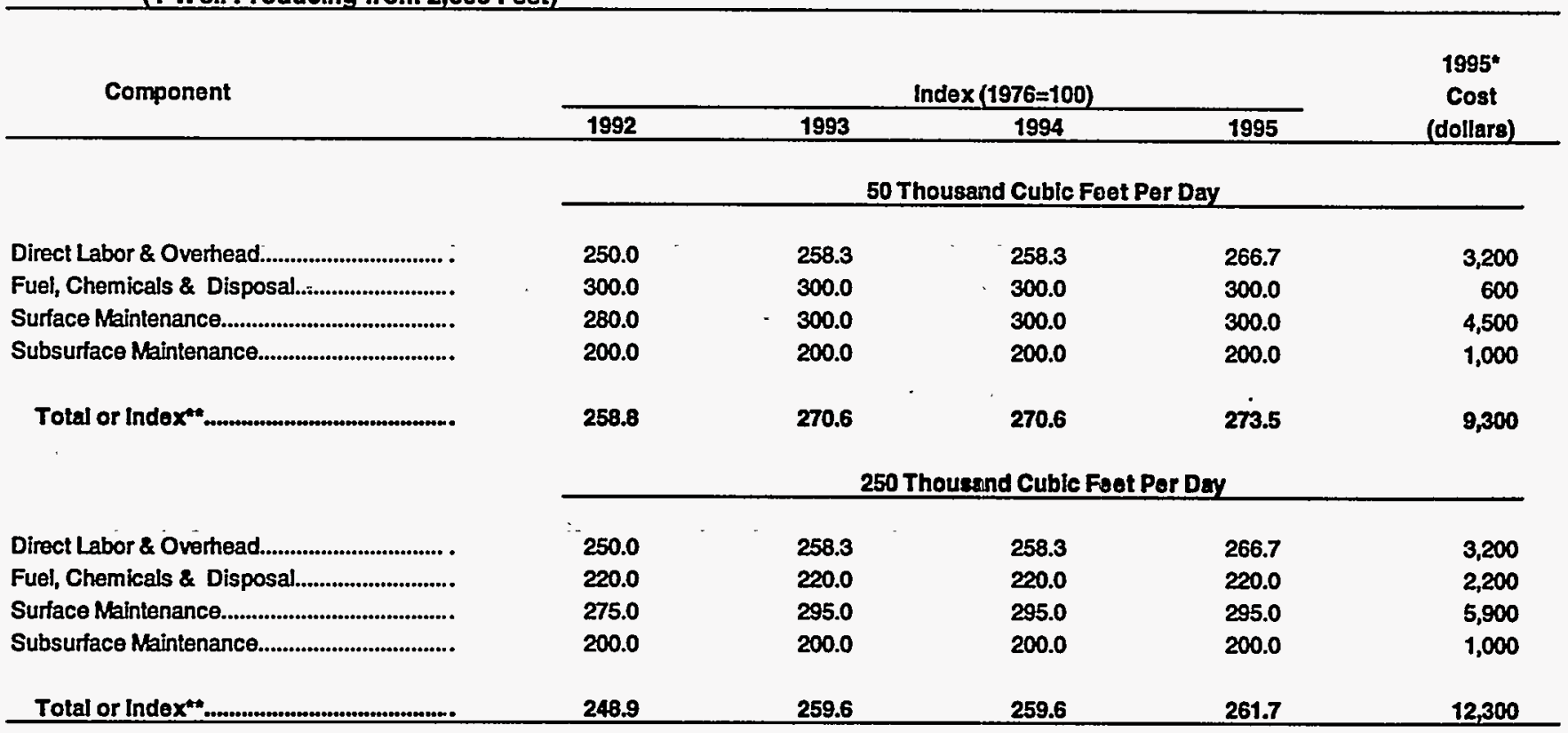


Table L7. Direct Annual Operating Costs and Indices for Gas Production in the Mid-Continent (1 Well Producing from 4,000 Feet)

\begin{tabular}{|c|c|c|c|c|c|}
\hline \multirow[t]{2}{*}{ Component } & \multicolumn{4}{|c|}{ Index $(1976=100)$} & \multirow{2}{*}{$\begin{array}{c}\text { 1995 } \\
\text { Cost } \\
\text { (dollars) }\end{array}$} \\
\hline & 1992 & 1993 & 1994 & 1995 & \\
\hline & \multicolumn{5}{|c|}{50 Thousand Cubic Feet Per Day } \\
\hline Direct Labor \& Overhead..................................... . & 242.9 & 250.0 & 250.0 & 257.1 & 3,600 \\
\hline 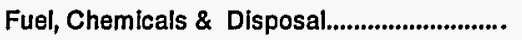 & 220.0 & 220.0 & 220.0 & 220.0 & 1,100 \\
\hline 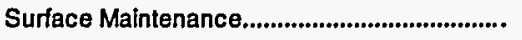 & 280.0 & 300.0 & 300.0 & 300.0 & 4,500 \\
\hline Subsurface Maintenance & 175.0 & 175.0 & 175.0 & 175.0 & 1,400 \\
\hline \multirow[t]{2}{*}{ Total or Index $x^{* *}$} & 240.5 & 250.0 & 250.0 & 252.4 & 10,600 \\
\hline & \multicolumn{5}{|c|}{250 Thousand Cuble Feet Per Day } \\
\hline Direct Labor \& Overhead..................................... . & 242.9 & 250.0 & 250.0 & 257.1 & 3,600 \\
\hline Fuel, Chemicals \& Disposal.................................. & 220.0 & 220.0 & 220.0 & 220.0 & 4,400 \\
\hline Surface Maintenance.......................................... & 275.0 & 291.7 & 287.5 & 287.5 & 6,900 \\
\hline Subsurface Maintenance..................................... & 175.0 & 175.0 & 175.0 & 175.0 & 1,400 \\
\hline \multirow[t]{2}{*}{ Total or Index $* * \ldots$} & 239.4 & 247.0 & 245.5 & 247.0 & 16,300 \\
\hline & \multicolumn{5}{|c|}{500 Thousand Cubic Feet Per Day } \\
\hline Direct Labor \& Overhead..................................... . & 242.9 & 250.0 & 250.0 & 257.1 & 3,600 \\
\hline Fuel, Chemicals \& Disposal.............................. & 263.2 & 278.9 & 278.9 & 278.9 & 5,300 \\
\hline 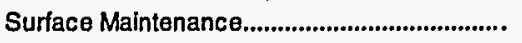 & 278.9 & 294.7 & 289.5 & 289.5 & 5,500 \\
\hline Subsurface Maintenance ....................................... & 175.0 & 175.0 & 175.0 & 175.0 & 1,400 \\
\hline Total or Index ${ }^{*} \ldots \ldots \ldots \ldots$ & 251.7 & 263.3 & 261.7 & 263.3 & 15,800 \\
\hline
\end{tabular}

Table L8. Direct Annual Operating Costs and Indices for Gas Production in the Mid-Continent (1 Well Producing from 8,000 Feet)

\begin{tabular}{|c|c|c|c|c|c|}
\hline \multirow[t]{2}{*}{ Component } & \multicolumn{4}{|c|}{ Index $(1976=100)$} & \multirow{2}{*}{$\begin{array}{c}\text { 1995* } \\
\text { Cost } \\
\text { (dollars) }\end{array}$} \\
\hline & 1992 & 1993 & 1994 & 1995 & \\
\hline & \multicolumn{5}{|c|}{ 250 Thousand Cubic Feet Per Day } \\
\hline Direct Labor \& Overhead................................. . & 243.8 & 256.3 & 256.3 & 256.3 & 4,100 \\
\hline Fuel, Chemicals \& Disposal........................... & 228.2 & 228.2 & 228.2 & 225.6 & 8,800 \\
\hline Surface Maintenance....................................... & 261.5 & 274.4 & 271.8 & 271.8 & 10,600 \\
\hline Subsurface Maintenance................................ & 175.0 & 175.0 & 175.0 & 175.0 & 2,100 \\
\hline \multirow[t]{2}{*}{ Total or Index ${ }^{* *} \ldots \ldots \ldots \ldots \ldots \ldots \ldots \ldots \ldots$} & 236.8 & 243.4 & 242.5 & 241.5 & 25,600 \\
\hline & \multicolumn{5}{|c|}{500 Thousand Cubic Feet Per Day } \\
\hline Direct Labor \& Overhead................................. . & 243.8 & 256.3 & 256.3 & 256.3 & 4,100 \\
\hline Fuel, Chemicals \& Disposal.......................... & 271.4 & 281.0 & 281.0 & 281.0 & 5,900 \\
\hline Surface Maintenance...................................... & 261.8 & 276.5 & 270.6 & 270.6 & 9,200 \\
\hline Subsurface Maintenance............................... & 175.0 & 175.0 & 175.0 & 175.0 & 2,100 \\
\hline Total or Index & 248.2 & 259.0 & 256.6 & 256.6 & 21,300 \\
\hline
\end{tabular}


Table L9. Direct Annual Operating Costs and Indices for. Gas Production in the Mid-Continent (1 Well Producing from 12,000 Feet)

\begin{tabular}{|c|c|c|c|c|c|}
\hline \multirow[t]{2}{*}{ Component } & \multicolumn{4}{|c|}{ Index $(1976=100)$} & \multirow{2}{*}{$\begin{array}{c}\text { 1995" } \\
\text { Cost } \\
\text { (dollars) }\end{array}$} \\
\hline & 1992 & 1993 & 1994 & 1995 & \\
\hline & \multicolumn{5}{|c|}{250 Thousand Cubic Feet Per Day } \\
\hline- & $\cdot$ & & & & \\
\hline Direct Labor \& Overhead................................... . & 247.4 & 263.2 & 257.9 & 263.2 & 5,000 \\
\hline Fuel, Chemicals \& Disposal............................ & 222.0 & 222.0 & 222.0 & 222.0 & 11,100 \\
\hline Surface Maintenance....................................... & 270.0 & 282.5 & 280.0 & 282.5 & 11,300 \\
\hline Subsurface Maintenance.................................... & 166.7 & 170.8 & 170.8 & 170.8 & 4,100 \\
\hline \multirow[t]{2}{*}{ Total or Index } & 230.1 & 236.8 & 235.3 & 236.8 & 31,500 \\
\hline & \multicolumn{5}{|c|}{500 Thousand Cubic Feet Per Day } \\
\hline Direct Labor \& Overhead................................ & 247.4 & 263.2 & 257.9 & 263.2 & 5,000 \\
\hline Fuel, Chemicals \& Disposal.............................. & 252.0 & 264.0 & 264.0 & 264.0 & 6,600 \\
\hline 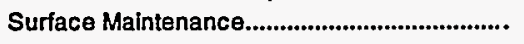 & 268.6 & 282.9 & 280.0 & 282.9 & 9,900 \\
\hline Subsurface Maintenance.................................. & 166.7 & 170.8 & 170.8 & 170.8 & 4,100 \\
\hline \multirow[t]{2}{*}{ Total or Index } & 236.9 & 248.5 & 246.6 & 248.5 & 25,600 \\
\hline & \multicolumn{5}{|c|}{1 Million Cubic Feet Per Day } \\
\hline Direct Labor \& Overhead.................................. . & 247.4 & 263.2 & 257.9 & 263.2 & 5,000 \\
\hline Fuel, Chemicals \& Disposal......................... & 258.8 & 268.6 & 268.6 & 266.7 & 13,600 \\
\hline 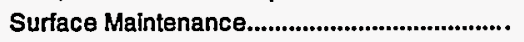 & 256.4 & 269.2 & 261.5 & 261.5 & 10,200 \\
\hline Subsurface Maintenance.................................... & 166.7 & 170.8 & 170.8 & 170.8 & 4,100 \\
\hline Total or Index & 239.8 & 250.4 & 247.4 & 247.4 & 32,900 \\
\hline
\end{tabular}

Table L10. Direct Annual Operating Costs and Indices for Gas Production in the Mid-Continent (1 Well Producing from 16,000 Feet)

\begin{tabular}{|c|c|c|c|c|c|}
\hline \multirow[t]{2}{*}{ - Component } & \multicolumn{4}{|c|}{ Index $(1976=100)$} & \multirow[t]{2}{*}{$\begin{array}{c}\text { 1995* } \\
\text { Cost } \\
\text { (dollars) }\end{array}$} \\
\hline & 1992 & 1993 & 1994 & 1995 & \\
\hline & \multicolumn{5}{|c|}{500 Thousand Cubic Feet Per Day } \\
\hline Direct Labor \& Overhead................................ . & 247.4 & 263.2 & 257.9 & 263.2 & 5,000 \\
\hline Fuel, Chemicals \& Disposal.......................... & 241.9 & 251.6 & 251.6 & 248.4 & 7,700 \\
\hline Surface Maintenance..................................... & 256.4 & $269.2^{-}$ & 261.5 & 261.5 & 10,200 \\
\hline Subsurface Maintenance.................................... & 169.0 & 172.4 & 172.4 & 172.4 & 5,000 \\
\hline \multirow[t]{2}{*}{ Total or Index* } & 229.7 & 239.8 & 236.4 & 236.4 & 27,900 \\
\hline & \multicolumn{4}{|c|}{1 Million Cubic Feet Per Day } & \\
\hline Direct Labor \& Overhead.................................. . & 247.4 & 263.2 & 257.9 & 263.2 & 5,000 \\
\hline Fuel, Chemicals \& Disposal............................. & 244.3 & 254.1 & 252.5 & 252.5 & 15,400 \\
\hline 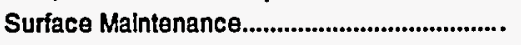 & 256.4 & 269.2 & 261.5 & 261.5 & 10,200 \\
\hline Subsurface Malntenance................................... & 169.0 & 172.4 & 172.4 & 172.4 & 5,000 \\
\hline \multirow[t]{2}{*}{ Total or Index } & 233.1 & 243.2 & 239.9 & 240.5 & 35,600 \\
\hline & \multicolumn{5}{|c|}{5 Million Cubic Foet Per Day } \\
\hline Direct Labor \& Overhead.................................. . & 247.4 & 263.2 & 257.9 & 263.2 & 5,000 \\
\hline Fuel, Chemicals \& Disposal.......................... & 147.4 & 152.6 & 152.6 & 152.6 & 14,800 \\
\hline Surface Maintenance..................................... & 265.3 & 275.5 & 269.4 & 273.5 & 13,400 \\
\hline Subsurface Maintenance................................ & 116.1 & 119.4 & 119.4 & 119.4 & 3,700 \\
\hline Total or Index & 181.6 & 188.8 & 186.7 & 188.3 & 36,900 \\
\hline
\end{tabular}


Table M1. Lease Equipment Costs and Indices for Gas Production in the Rocky Mountains (1 Well Producing from 2,000 Feet)

\begin{tabular}{|c|c|c|c|c|c|}
\hline \multirow[t]{2}{*}{ Component } & \multicolumn{4}{|c|}{ Index $(1976=100)$} & \multirow{2}{*}{$\begin{array}{c}\text { 1995 } \\
\text { Cost } \\
\text { (dollars) }\end{array}$} \\
\hline & 1992 & 1993 & 1994 & 1995 & \\
\hline & \multicolumn{5}{|c|}{ 50 Thousand Cuble Feet Per Day } \\
\hline Flowlines and Connections.......................... & 157.1 & 171.4 & 171.4 & 178.6 & 2,500 \\
\hline Production Package........................................ . & 140.5 & 145.2 & 150.0 & 150.0 & 6,300 \\
\hline Storage Tanks................................................ & 182.1 & 191.1 & 173.2 & 183.9 & 10,300 \\
\hline \multirow[t]{2}{*}{ Total or Index..................................... } & 163.4 & 171.4 & 164.3 & 170.5 & 19,100 \\
\hline & \multicolumn{5}{|c|}{ 250 Thousand Cuble Feet Per Day } \\
\hline Flowlines and Connections........................... . & 157.1 & 171.4 & 171.4 & 178.6 & 2,500 \\
\hline Production Package........................................ . & 140.5 & 145.2 & 150.0 & 150.0 & 6,300 \\
\hline Storage Tanks............................................... & 182.1 & 191.1 & 173.2 & 183.9 & 10,300 \\
\hline Total or Index.......................................... & 163.4 & 171.4 & 164.3 & 170.5 & 19,100 \\
\hline
\end{tabular}

Table M2. Lease Equipment Costs and Indices for Gas Production in the Rocky Mountains (1 Well Producing from 4,000 Feet)

\begin{tabular}{|c|c|c|c|c|c|}
\hline \multirow{2}{*}{ Component } & \multicolumn{4}{|c|}{ Index $(1976=100)$} & \multirow{2}{*}{$\begin{array}{c}1995^{*} \\
\text { Cost } \\
\text { (dollars) }\end{array}$} \\
\hline & 1992 & 1993 & 1994 & 1995 & \\
\hline & \multicolumn{5}{|c|}{50 Thousand Cubic Feet Per Day } \\
\hline Flowlines and Connectlons........................... . & 157.1 & 171.4 & 171.4 & 178.6 & 2,500 \\
\hline Production Package...................................... & 140.5 & 145.2 & 150.0 & 150.0 & 6,300 \\
\hline Storage Tanks.................................................. & 182.1 & 191.1 & 173.2 & 183.9 & 10,300 \\
\hline \multirow[t]{2}{*}{ Total or Index............................................. } & 163.4 & 171.4 & 164.3 & 170.5 & 19,100 \\
\hline & \multicolumn{5}{|c|}{250 Thousand Cubic Feet Per Day } \\
\hline Flowlines and Connectlons........................... & 208.7 & 215.2 & 184.8 & 197.8 & 9,100 \\
\hline Production Package......................................... . & 156.8 & 162.2 & 164.9 & 167.6 & 6,200 \\
\hline 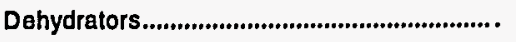 & 178.1 & 182.3 & 157.3 & 156.3 & 15,000 \\
\hline 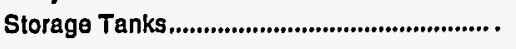 & 182.1 & 191.1 & 173.2 & 183.9 & 10,300 \\
\hline \multirow[t]{2}{*}{ Total or Index......................................... } & 181.7 & 187.7 & 167.7 & 172.8 & 40,600 \\
\hline & \multicolumn{5}{|c|}{500 Thousand Cuble Feet Per Day } \\
\hline Flowlines and Connectlons........................... . & 223.5 & 226.5 & 185.3 & 200.0 & 6,800 \\
\hline Production Package......................................... . & 147.0 & 150.0 & 109.0 & 109.0 & 10,900 \\
\hline 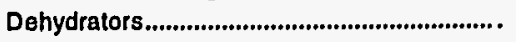 & 178.1 & 182.3 & 157.3 & 156.3 & 15,000 \\
\hline Storage Tanks................................................. & 182.1 & 191.1 & 173.2 & 183.9 & 10,300 \\
\hline Total or Index.......................................... & 173.4 & 178.0 & 146.9 & 150.3 & 43,000 \\
\hline
\end{tabular}


Table M3. Lease Equipment Costs and Indices for Gas Productlon in the Rocky Mountains (1 Well Producing from 8,000 Feet)

\begin{tabular}{|c|c|c|c|c|c|}
\hline \multirow[t]{2}{*}{ Component } & \multicolumn{4}{|c|}{ Index $(1976=100)$} & \multirow{2}{*}{$\begin{array}{c}1995^{*} \\
\text { Cost } \\
\text { (dollars) }\end{array}$} \\
\hline & 1992 & 1993 & 1994 & 1995 & \\
\hline & \multicolumn{5}{|c|}{ 250 Thousand Cubic Feet Per Day } \\
\hline Flowlines and Connections............................. . & 193.2 & 193.2 & 168.2 & 179.5 & 7,900 \\
\hline Production Package......................................... . & 147.0 & 150.0 & 109.0 & 109.0 & 10,900 \\
\hline 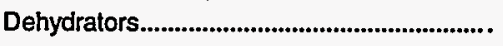 & 178.1 & 182.3 & 157.3 & 156.3 & 15,000 \\
\hline Storage Tanks............................................. . & 182.1 & 191.1 & 173.2 & 183.9 & 10,300 \\
\hline \multirow[t]{2}{*}{ Total or Index.......................................... } & 170.6 & 174.7 & 145.6 & 149.0 & 44,100 \\
\hline & \multicolumn{5}{|c|}{500 Thousand Cubic Feet Per Day } \\
\hline \multicolumn{6}{|l|}{ Flowlines and Connections............................. . } \\
\hline Production Package............................................ & 216.7 & 219.4 & 186.1 & 200.0 & 7,200 \\
\hline 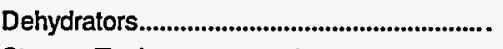 & 147.0 & 150.0 & 109.0 & 109.0 & 10,900 \\
\hline \multirow[t]{2}{*}{ 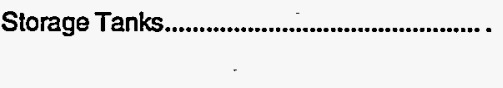 } & 178.1 & 182.3 & 157.3 & 156.3 & 15,000 \\
\hline & 182.1 & 191.1 & 173.2 & 183.9 & 10,300 \\
\hline Total or Index......................................... & 172.9 & 177.4 & 147.2 & 150.7 & 43,400 \\
\hline
\end{tabular}

Table M4. Lease Equipment Costs and Indices for Gas Production in the Rocky Mountains (1 Well Producing from 12,000 Feet)

\begin{tabular}{|c|c|c|c|c|c|}
\hline \multirow[t]{2}{*}{ Component } & \multicolumn{4}{|c|}{ Index $(1976=100)$} & \multirow{2}{*}{$\begin{array}{c}\text { 1995* } \\
\text { Cost } \\
\text { (dollar8) }\end{array}$} \\
\hline & 1992 & 1993 & 1994 & 1995 & \\
\hline & \multicolumn{5}{|c|}{250 Thousand Cublc Feet Per Day } \\
\hline Flowlines and Connections............................. & 290.0 & 290.0 & 291.7 & 313.3 & 18,800 \\
\hline Production Package........................................... . . & 147.0 & 150.0 & 109.0 & 109.0 & 10,900 \\
\hline Dehydrators.................................................... & 178.1 & 182.3 & 157.3 & 156.3 & 15,000 \\
\hline Storage Tanks.................................................. & 182.1 & 191.1 & 173.2 & 183.9 & 10,300 \\
\hline \multirow[t]{2}{*}{ 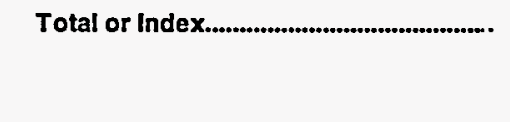 } & 190.4 & 194.2 & 170.5 & 176.3 & 55,000 \\
\hline & \multicolumn{5}{|c|}{500 Thousand Cuble Feet Per Day } \\
\hline Flowlines and Connections............................ & 321.2 & 323.1 & 323.1 & 348.1 & 18,100 \\
\hline Production Package............................................. . & 147.0 & 150.0 & 109.0 & 109.0 & 10,900 \\
\hline 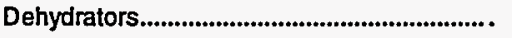 & 178.1 & 182.3 & 157.3 & 156.3 & 15,000 \\
\hline Storage Tanks.................................................... & 182.1 & 191.1 & 173.2 & 183.9 & 10,300 \\
\hline \multirow[t]{2}{*}{ Total or Index............................................. } & 193.1 & 197.4 & 172.7 & 178.6 & 54,300 \\
\hline & \multicolumn{5}{|c|}{1 Million Cubic Feet Per Day } \\
\hline Flowlines and Connections............................. . & 321.2 & 323.1 & 323.1 & 348.1 & 18,100 \\
\hline Production Package........................................... . & 147.0 & 150.0 & 109.0 & 109.0 & 10,900 \\
\hline 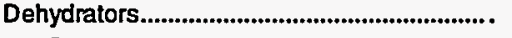 & 178.1 & 182.3 & 157.3 & 156.3 & 15,000 \\
\hline Storāge Tanks.................................................. . & 182.1 & 191.1 & 173.2 & 183.9 & 10,300 \\
\hline 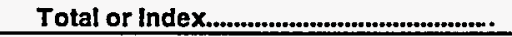 & 193.1 & 197.4 & 172.7 & 178.6 & 54,300 \\
\hline
\end{tabular}


Table M5. Direct Annual Operating Costs and Indices for Gas Production in the Rocky Mountains (1 Well Producing from 2,000 Feet)

\begin{tabular}{|c|c|c|c|c|c|}
\hline \multirow[t]{2}{*}{ Component } & \multicolumn{4}{|c|}{ Index $(1976=100)$} & \multirow{2}{*}{$\begin{array}{c}1995^{\star} \\
\text { Cost } \\
\text { (dollars) }\end{array}$} \\
\hline & 1992 & 1993 & 1994 & 1995 & \\
\hline & \multicolumn{5}{|c|}{50 Thousand Cubic Feet Per Day } \\
\hline Direct Labor \& Overhead................................. . & 253.8 & 261.5 & 261.5 & 261.5 & 3,400 \\
\hline Fuel, Chemicals \& Disposal............................ & 300.0 & 300.0 & 300.0 & 300.0 & 600 \\
\hline Surlace Maintenance.......................................... & 203.7 & 207.4 & 207.4 & 207.4 & 5,600 \\
\hline Subsurface Maintenance.................................. & 133.3 & 133.3 & 133.3 & 133.3 & 800 \\
\hline \multirow[t]{2}{*}{ Total or Index } & 212.5 & 216.7 & 216.7 & 216.7 & 10,400 \\
\hline & \multicolumn{5}{|c|}{250 Thousand Cubic Feet Per Day } \\
\hline Direct Labor \& Overhead................................. . . & 253.8 & 261.5 & 261.5 & 261.5 & 3,400 \\
\hline Fuel, Chemicals \& Disposal............................. & 220.0 & 220.0 & 220.0 & 220.0 & 2,200 \\
\hline 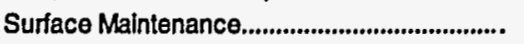 & 203.7 & 207.4 & 207.4 & 207.4 & 5,600 \\
\hline Subsurface Maintenance.................................. & 133.3 & 133.3 & 133.3 & 133.3 & 800 \\
\hline Total or Index $x^{* *}$ & 210.7 & 214.3 & 214.3 & 214.3 & 12,000 \\
\hline
\end{tabular}

Table M6. Direct Annual Operating Costs and Indices for Gas Production In the Rocky Mountains (1 Well Producing from 4,000 Feet)

\begin{tabular}{|c|c|c|c|c|c|}
\hline \multirow[t]{2}{*}{ Component } & \multicolumn{4}{|c|}{ Index $(1976=100)$} & \multirow{2}{*}{$\begin{array}{c}\text { 1995" } \\
\text { Cost } \\
\text { (dollars) }\end{array}$} \\
\hline & 1992 & 1993 & 1994 & 1995 & \\
\hline & \multicolumn{5}{|c|}{50 Thousand Cuble Feet Per Day } \\
\hline Direct Labor \& Overhead................................ . & 246.7 & 253.3 & 260.0 & 260.0 & 3,900 \\
\hline Fuel, Chemicals \& Dlsposal........................... & 220.0 & 220.0 & 220.0 & 220.0 & 1,100 \\
\hline Surface Maintenance....................................... & 203.7 & 207.4 & 207.4 & 207.4 & 5,600 \\
\hline Subsurface Maintenance................................. & 155.6 & 155.6 & 155.6 & 166.7 & 1,500 \\
\hline \multirow[t]{2}{*}{ Total or Index } & 208.9 & 212.5 & 214.3 & 216.1 & 12,100 \\
\hline & \multicolumn{5}{|c|}{250 Thousand Cublc Feet Per Day } \\
\hline Direct Labor \& Ovemead................................. . & 246.7 & 253.3 & 260.0 & 260.0 & 3,900 \\
\hline Fuel, Chemicals \& Disposal.......................... & 220.0 & 225.0 & 225.0 & 225.0 & 4,500 \\
\hline Surface Maintenance...................................... & 206.1 & 212.2 & 208.2 & 210.2 & 10,300 \\
\hline Subsurface Maintenance................................ & 155.6 & 155.6 & 155.6 & 166.7 & 1,500 \\
\hline \multirow[t]{2}{*}{ 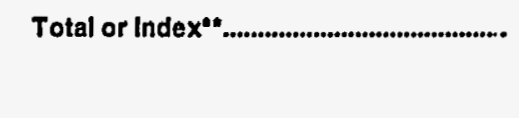 } & 210.8 & 216.1 & 215.1 & 217.2 & 20,200 \\
\hline & \multicolumn{5}{|c|}{ 500 Thousand Cubic Feet Per Day } \\
\hline Dlrect Labor \& Overhead................................. . & 246.7 & 253.3 & 260.0 & 260.0 & 3,900 \\
\hline Fuel, Chemicals \& Disposal............................ & 216.0 & 232.0 & 236.0 & 236.0 & 5,900 \\
\hline Surface Maintenance....................................... & 191.3 & 197.8 & 193.5 & 193.5 & 8,900 \\
\hline Subsurface Maintenance............................... & 155.6 & 155.6 & 155.6 & 166.7 & 1,500 \\
\hline Total or Index $\ldots$ & 203.2 & 211.6 & 211.6 & 212.6 & 20,200 \\
\hline
\end{tabular}


Table M7. Direct Annual Operating Costs and Indices for Gas Production in the Rocky Mountains (1 Well Producing from 8,000 Feet)

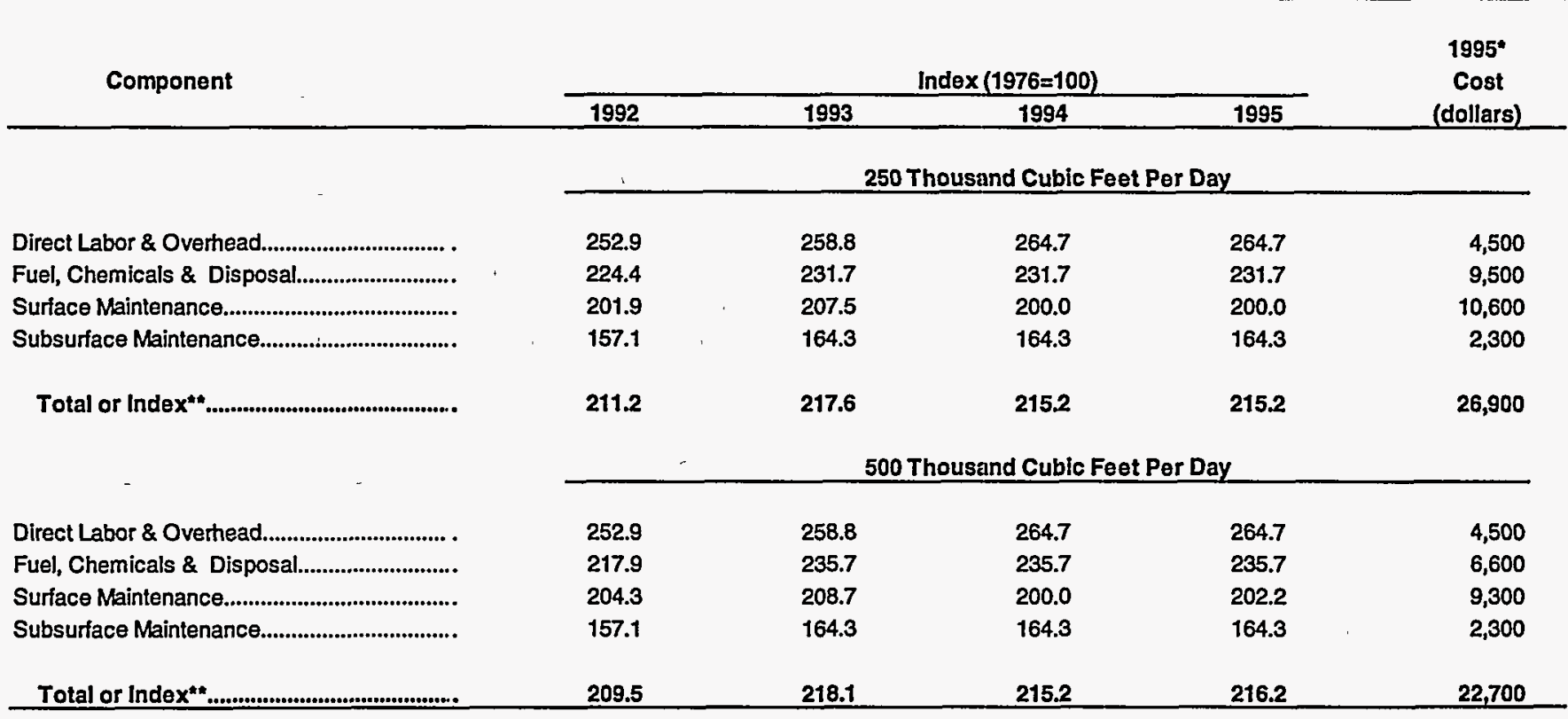

Table M8. Direct Annual Operating Costs and Indices for Gas Production in the Rocky Mountains (1 Well Producing from 12,000 Feet)

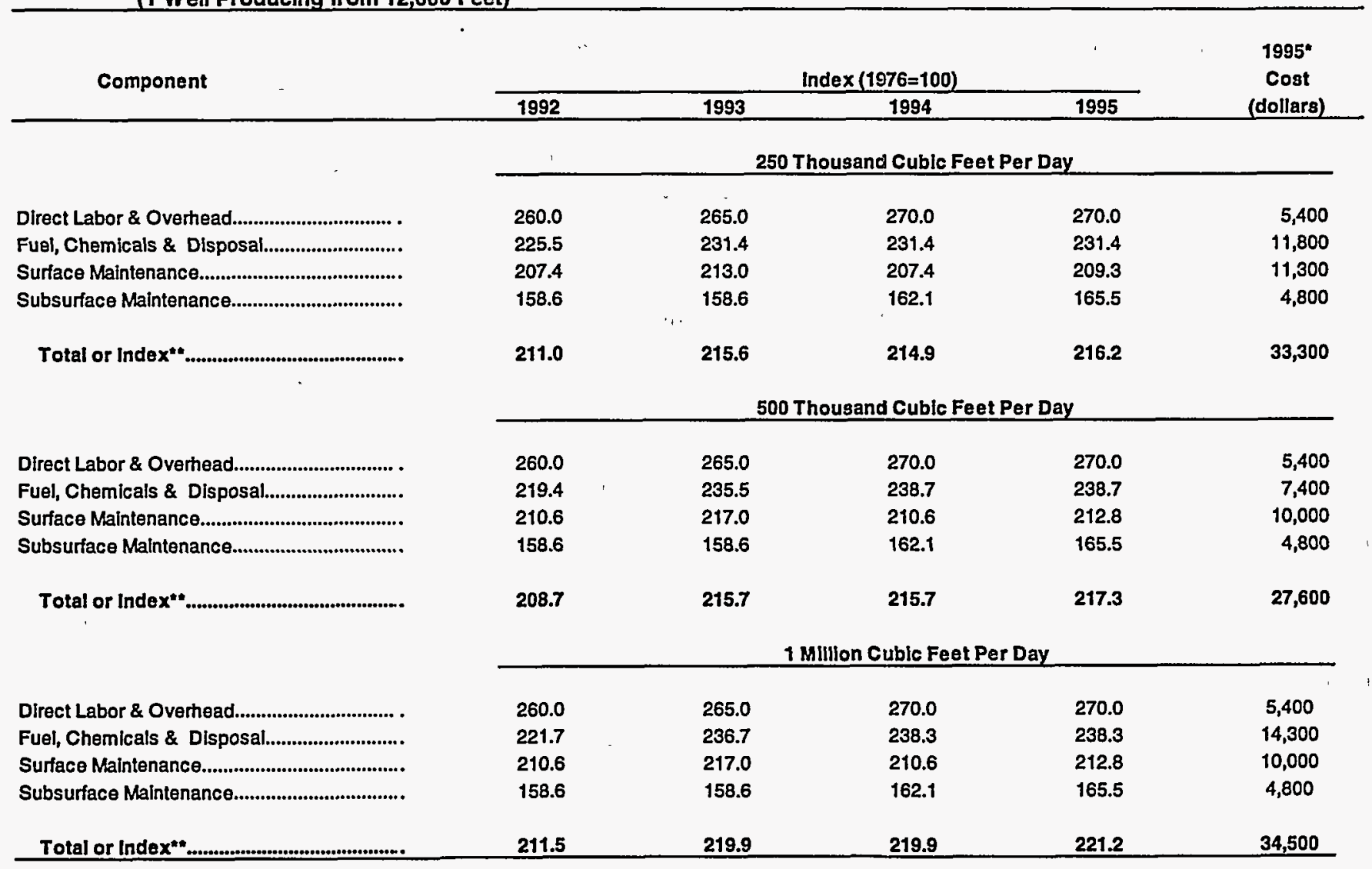




\section{Section III}

\section{Appendix N}

Equipping and Operating Cost Indices and Other Economic Indicators 


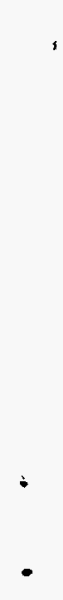




\section{Appendix $\mathbf{N}$}

\section{Equipping and Operating Cost Indices and Other Economic Indicators}

Appendix $\mathrm{N}$ contains a general overview of oil and gas economics from 1976 through 1995.

Unweighted aggregates of equipping and operating costs from the summary tables were indexed with 1976 as the base year. The Gross Domestic Product (GDP) Implicit Price Deflator was used to deflate these indices and the Producer Price Indices (PPI). Each deflated index would equal 100 if the change in cost matched the change in the GDP for that index. The results appear in Tables N1 and N2, and are illustrated in Figures N1 and N2.

Although the aggregate average costs may not be the average costs for all oil and gas wells in the United States, it is possible to make some meaningful observations.

The deflated indices for oil lease equipment peaked in 1981 at 120.5 and continued in a general decline to a low of 70 in 1994. New equipment for oil leases is competing with used equipment which explains why new equipment costs are still more than 25 percent below the deflated costs of 1976. Volatile tubing prices have been the largest part of the changing equipment costs for much of the time, but are less so than non-tubing equipment costs in the period, 1992-1995. Gas well equipment prices, as shown in Figure N1, changed less than oil equipment prices, but only slightly. Equipment price trends are not easily described as "upward" in spite of the increase from 1994 to 1995 .

The deflated indices for operating costs for both oil and gas leases peaked in 1982 at 142.5 and 120.6, respectively, and declined 28 percent for oil leases and 27 percent for gas leases by 1995 . This decline was primarily a reflection of the decrease in drilling activity and workovers which caused the service companies to cut prices and their own costs drastically to stay in business. Prices are expected to stabilize in 1996 for both drilling and workover activity, and may increase more rapidly than was true for other oil related activities in the 1992-1995 period. 
Table N1. Indices and Gross Domestic Product Deflated Indices of the Aggregate Average Equipping Costs for $O$ il and Gas Fields and the Producer Price Index (PPI) (Capital Equipment)

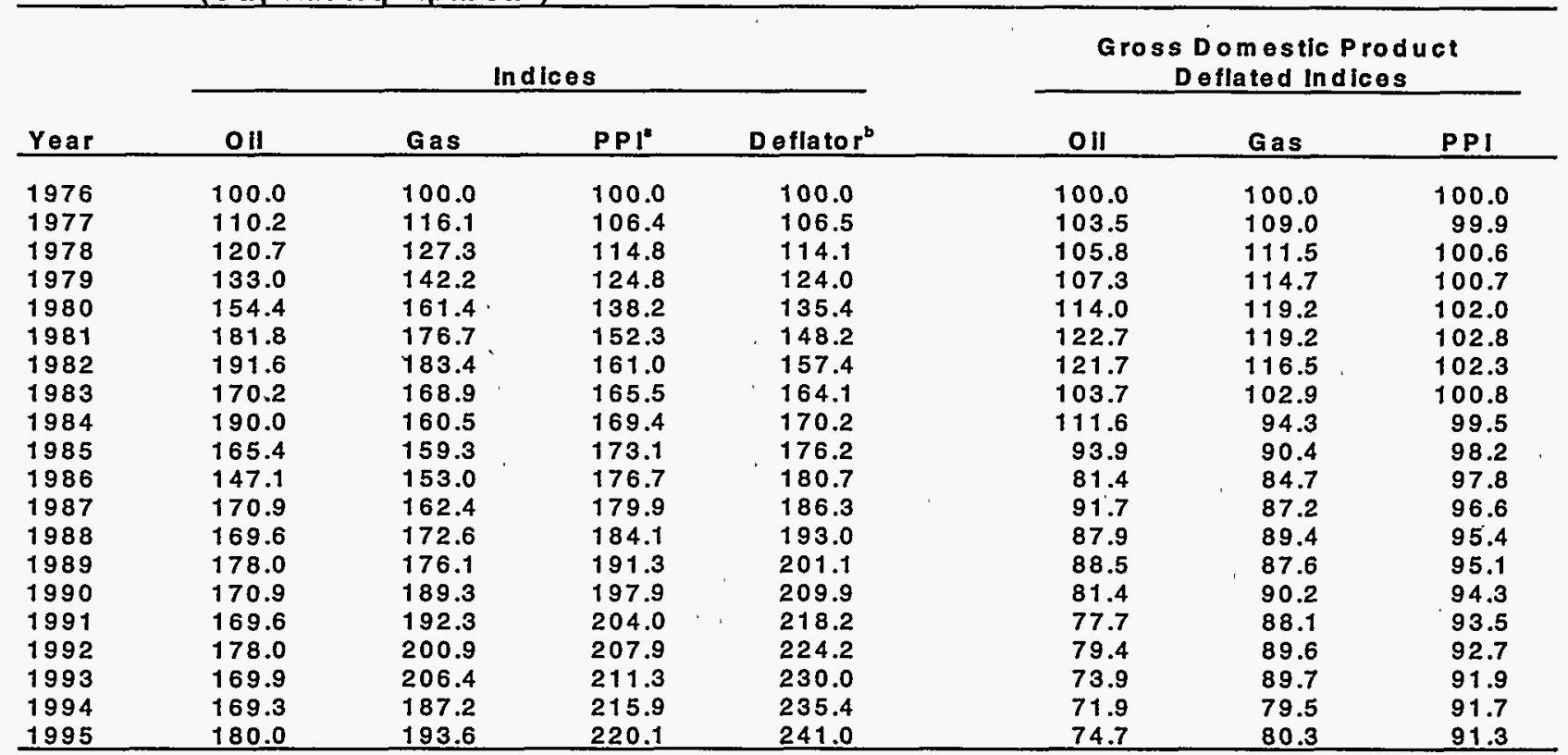

Producer P rlce Index (Capltal Equipment) obtained from the Bureau of Labor Statistics, U.S. Department of Labor.

'Gross Domestic Implicit Price Deflators were obtained from the Bureau of Economic Analysis, U.S. Department of Commerce. Notes: The aggregate average costs are the average of the costs from summary Tables 1 and 6 and do not represent the average costs of all wells in the United States.

Source: Energy information Administration, Office of Oll and $G$ as.

Table N2. The Gross Domestic Product Impllcit Price Deflator and the Gross Domestlc Product Deflated Indices of Operating Costs for Oll and Gas Flelds

\begin{tabular}{|c|c|c|c|c|c|}
\hline \multirow[b]{2}{*}{ Year } & \multirow{2}{*}{$\begin{array}{l}\text { GDP Implicit } \\
\text { Price Deflator " }\end{array}$} & \multicolumn{2}{|c|}{ Indices } & \multicolumn{2}{|c|}{$\begin{array}{l}\text { Gross Domestic Produc } \\
\text { Deflated Indices }\end{array}$} \\
\hline & & Oil & Gas & Oil & Gas \\
\hline $\begin{array}{l}1976 \\
1977 \\
1978 \\
1979 \\
1980 \\
1981 \\
1982 \\
1983 \\
1984 \\
1985 \\
1986 \\
1987 \\
1988 \\
1989 \\
1990 \\
1991 \\
1992 \\
1993 \\
1994 \\
1995\end{array}$ & $\begin{array}{l}100.0 \\
106.5 \\
114.1 \\
124.0 \\
135.4 \\
148.2 \\
157.4 \\
164.1 \\
170.2 \\
176.2 \\
180.7 \\
186.3 \\
193.0 \\
201.1 \\
209.9 \\
218.2 \\
224.2 \\
230.0 \\
235.4 \\
241.0\end{array}$ & $\begin{array}{l}100.0 \\
117.5 \\
130.3 \\
144.0 \\
174.2 \\
204.2 \\
228.4 \\
226.2 \\
230.1 \\
232.2 \\
212.9 \\
210.5 \\
220.1 \\
229.1 \\
243.4 \\
228.4 \\
233.3 \\
242.4 \\
256.2 \\
254.6\end{array}$ & $\begin{array}{l}100.0 \\
114.6 \\
121.8 \\
135.8 \\
156.4 \\
181.5 \\
193.2 \\
190.8 \\
192.0 \\
190.7 \\
182.1 \\
172.9 \\
181.4 \\
186.4 \\
201.9 \\
204.7 \\
208.5 \\
216.0 \\
216.0 \\
217.9\end{array}$ & $\begin{array}{l}100.0 \\
110.3 \\
114.2 \\
116.1 \\
128.6 \\
137.8 \\
145.1 \\
137.8 \\
135.2 \\
131.8 \\
117.8 \\
113.0 \\
114.0 \\
113.9 \\
116.0 \\
104.7 \\
104.0 \\
105.4 \\
108.8 \\
105.6\end{array}$ & $\begin{array}{r}100.0 \\
107.6 \\
106.7 \\
109.5 \\
115.5 \\
122.5 \\
122.7 \\
116.3 \\
112.8 \\
108.2 \\
100.8 \\
92.8 \\
94.0 \\
92.7 \\
96.2 \\
93.8 \\
93.0 \\
93.9 \\
91.8 \\
90.4\end{array}$ \\
\hline
\end{tabular}

"Gross Domestic Product Implicit Price Deflators were obtained from the Bureau of Economic Analysis, U.S. Department of Commerce. Notes: The aggregate average costs are the average of the costs from summary Tables 3 and 14 and do not represent the average costs of all wells in the United States.

Source: Energy Intormation Administration. Otflce of Oll and Gas. 
Figure N1. Gross Domestic Product Deflated Producer Price Indices, and Oil and Gas Field Equipping Cost Indices

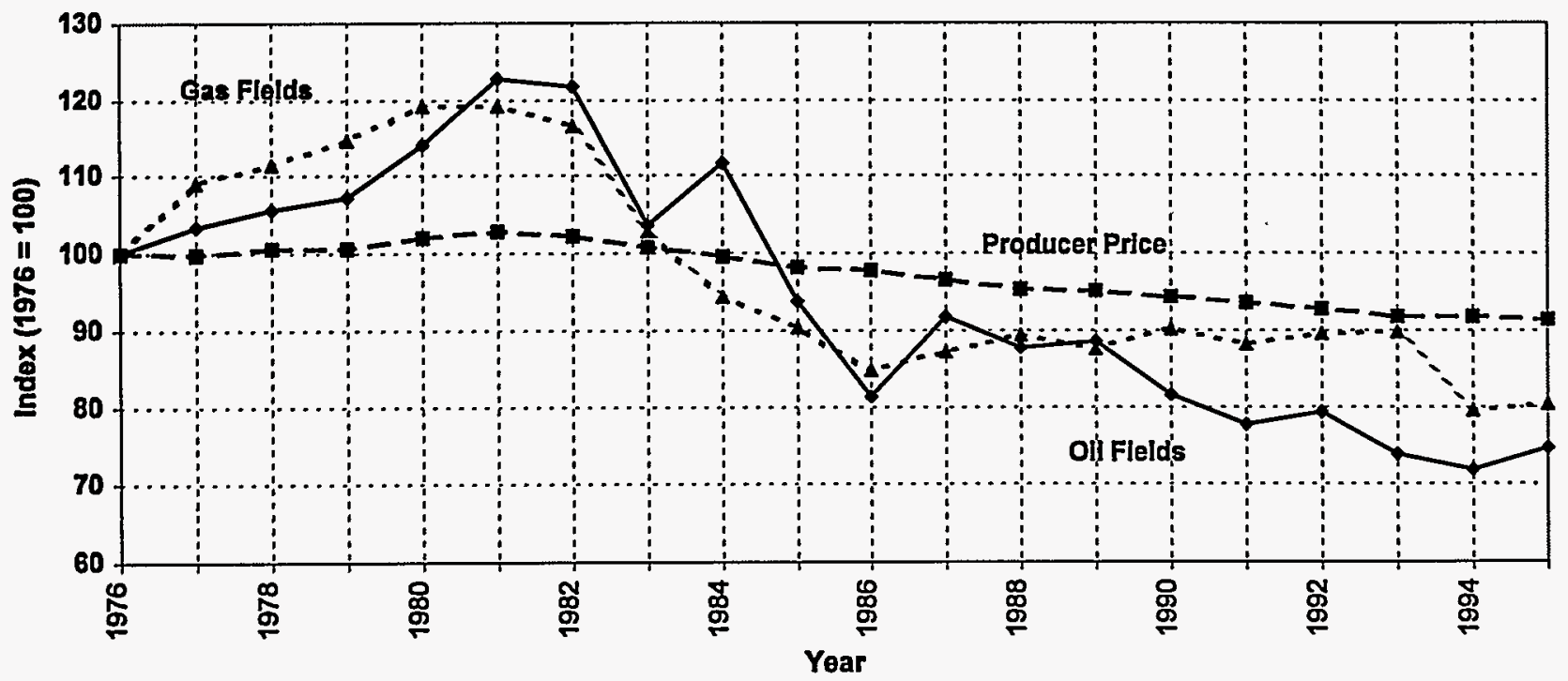

Source: Tablo $\mathbf{N 1 .}$

Figure N2. Gross Domestic Product Deflated Operating Cost Indices for Oil and Gas Fields

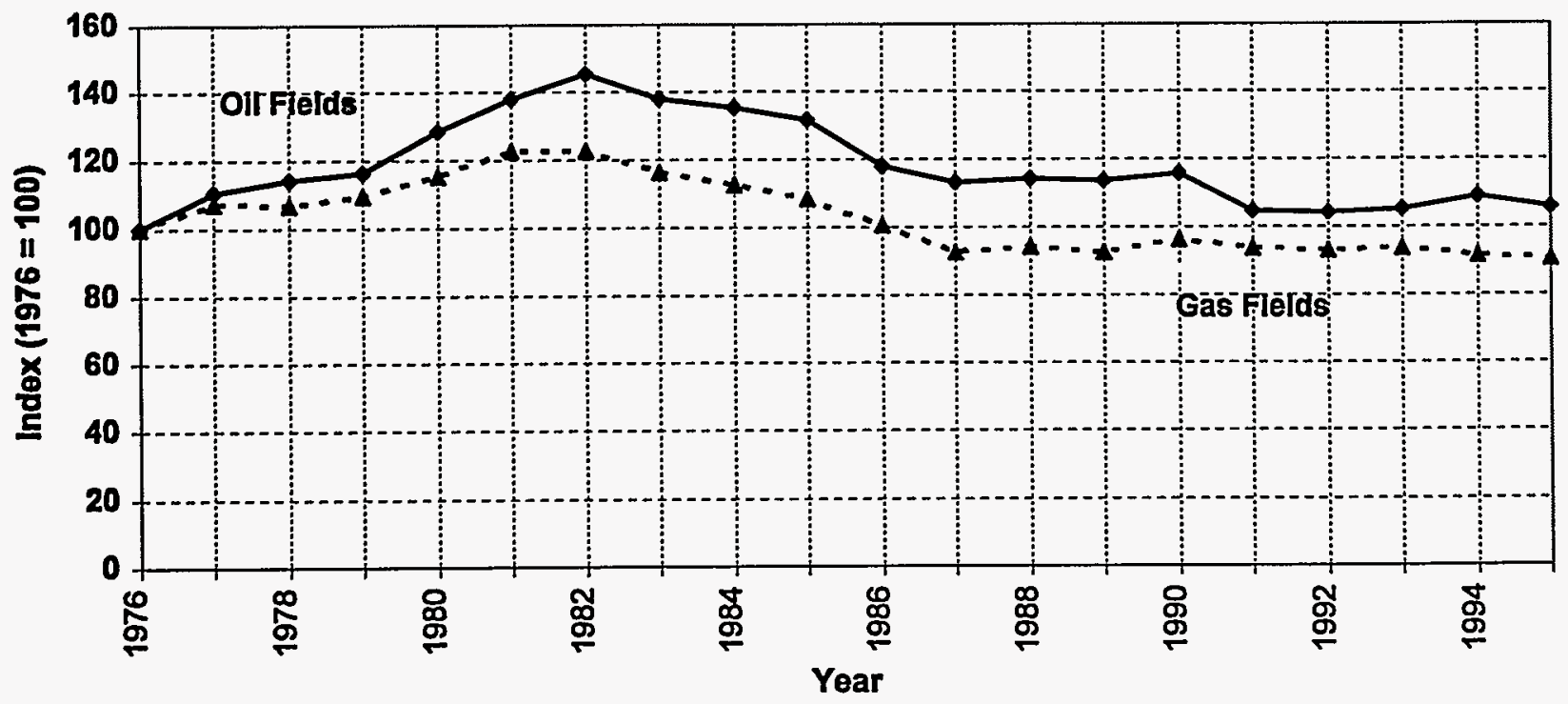

Sourco: Table N2. 


$$
\because \quad \cdot \%
$$




\section{Glossary}

Additional oil recovery: Recovery which follows primary, or natural depletion recovery, and is usually based on the application of processes which involve capital expenditures.

ad valorem: The basis for taxation of oil and gas operating properties, usually computed by expert assessment of current value.

API: American Petroleum Institute.

EIA: Energy Information Administration.

IPAA: Independent Petroleum Association of America.

JAS: Joint Association Survey, a survey of the cost of drilling wells in the U.S., conducted by the API, IPAA and MCOGA.

LACT: Lease automatic custody transfer, generally refers to unattended metering of oil sales from leases.

Mcf: One thouand (standard) cubic feet.

MCOGA: Mid-Continent Oil and Gas Association, one of a number of regional associations of independent oil and gas operators.

Natural depletion: Means of recovering oil or gas relying on natural pressure in the reservoir rocks to expel substances to surface facilities for treatment and sale.

Secondary recovery: See additional recovery. One common type is by means of water injection (waterflood).

Stripper well: A well that produces $90 \mathrm{Mcf}$ per day or less of gas-well gas for a period of three consecutive months while producing at its maximum rate of flow or an oil well which produces less than 15 barrels of oil per day at its maximum rate of production for a period of three consecutive months.

WSU: Well service unit. Equipment used to maintain oil and gas wells. Usually mounted on vehicles for movement over roads. 


\section{Introducing the Energy InfoDisc...}

\section{How much oil does the U.S. import from the Persian Gulf?}

How many U.S. households use personal computers?

\section{What's the long range outlook for nuclear power, and how will it affect other fuels?}

\section{How are clean air regulations affecting emissions in the electric power industry?}

Find answers to all your energy questions with Energy InfoDisc, the new CD-ROM providing the most comprehensive collection of energy information and analysis tools available today. Energy InfoDisc is a valuable resource for industry and government analysts, forecasters, journalists, and academic researchers - almost anyone needing access to current or historical energy information.

The Energy InfoDisc comes with advanced search capability to find the information you need in seconds - from over 180 of the most current periodicals and analytical publications of the Energy Information Administration (EIA), the independent statistical and analytical agency within the U.S. Department of Energy. With Energy InfoDisc, you also have instant access to EIA's wide range of national energy databases and special analysis applications. Don't be without this important resource; subscribe today.

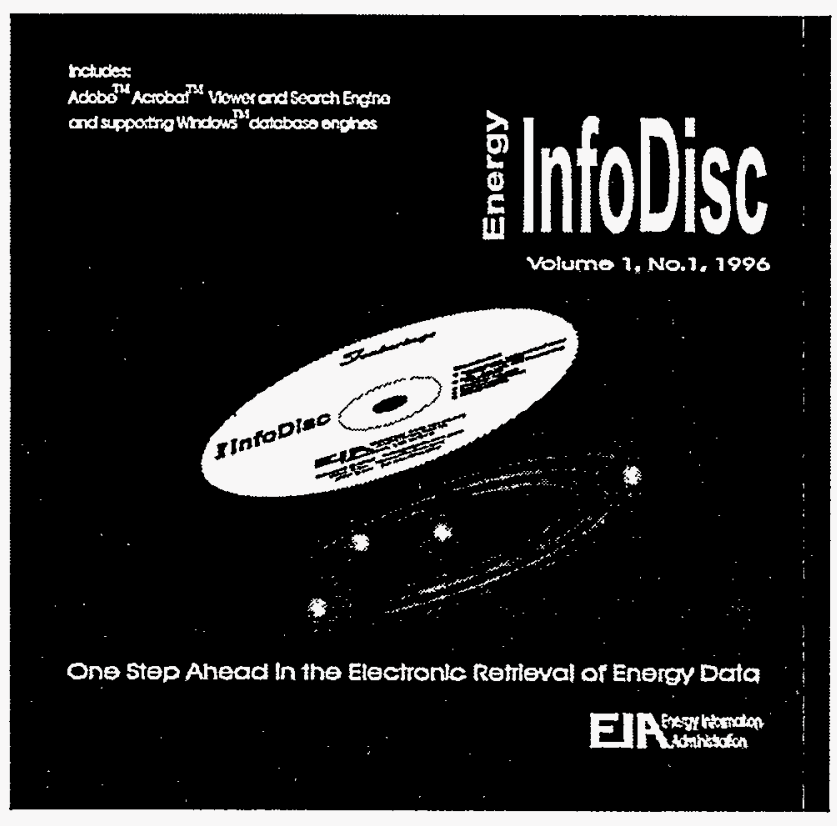

NOTE: Documents can be accessed using either Microsoft Windows or Macintosh System 7 operating systems. Databases and applications can only be accessed using Microsoft Windows.

\section{STAT-USA Order Form}

Yes, enter my order for the Energy InfoDisc CD-ROM:

Number of copies:

Single issue (U.S., Canada, \& Mexico): \$45

Single issue outside the U.S., Canada \& Mexico: $\$ 60$

Quarterly subscription in the U.S., Canada, \& Mexico: $\$ 100$

Quarterly subscription outside the U.S., Canada

\& Mexico: $\$ 160$

Contact or personal name

(please type or print)

\section{Company}

\section{street address}

Additional address

City, State, ZIP Code

Daytime phone
Charge your order

It's easy!

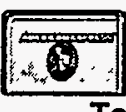

icove

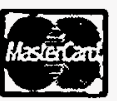

VISA

To fax your orders (202) 482-2164

To phone your orders (202)482-1986

For privacy protection, check the box below:

$\square$ Do not make my name available to requesters.

Check method of payment:

$\square$ AMEX $\square$ Discover $\square$ MasterCard $\square$ VISA .

(expiration date)

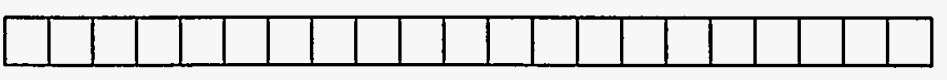

Thank you for your order!

Authorizing signature

$3 / 96$
Check payable to STAT-USA

Mail to: U.S. Department of Commerce

STAT-USA, Room H-4885

Washington, DC 20230 